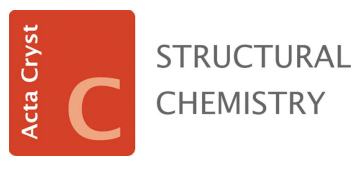

ISSN 2053-2296

Received 27 July 2020

Accepted 19 February 2021

Edited by M. Rosales-Hoz, Cinvestav, Mexico

Keywords: crystal structure; metal organic; three-dimensional structure; ferrocene; phosphine sulfide; chirality; planar chirality.

CCDC references: $1960106 ; 1960104$; $1960103 ; 1960105 ; 1943185 ; 1943184$

Supporting information: this article has supporting information at journals.iucr.org/c

\section{Synthesis and characterization of enantiopure planar-chiral phosphorus-linked diferrocenes}

\author{
Philipp Honegger, ${ }^{a *}$ Alexander Roller ${ }^{\mathrm{b}}$ and Michael Widhalm ${ }^{\mathrm{c}}$
}

${ }^{\text {a} D e p a r t m e n t ~ o f ~ C o m p u t a t i o n a l ~ B i o l o g i c a l ~ C h e m i s t r y, ~ U n i v e r s i t y ~ o f ~ V i e n n a, ~ W a e h r i n g e r ~ S t r a s s e ~ 17, ~ A-1090 ~ V i e n n a, ~}$
Austria, ${ }^{\mathbf{b}}$ Department of Inorganic Chemistry, Faculty of Chemistry, University of Vienna, Waehringer Strasse 42, A-1090
Vienna, Austria, and ${ }^{\mathbf{c}}$ Department of Chemical Catalysis, Faculty of Chemistry, University of Vienna, Waehringer Strasse
38, A-1090 Vienna, Austria. * ${ }^{*}$ Correspondence e-mail: philipp.honegger@univie.ac.at

In the course of an ongoing synthetic project on cyclic diferrocenylphosphines, we obtained a group of planar-chiral diferrocenyl compounds useful as precursors for subsequent cyclization. Here we report the crystal structures of two symmetric compounds $\left[\left(\mathrm{Fc}^{\mathrm{A}}\right)_{2}(\mathrm{Ph}) \mathrm{P}\right]$, one of which contains four stereogenic centres (two $\mathrm{C}$ chiral and two planar chiral centres), i.e. 1,1'-(phenylphosphanediyl)bis $\left\{\left(2 S_{\mathrm{p}}\right)-2-[(1 R)-1\right.$-(acetyloxy)ethyl]ferrocene $\}$, $\left[\mathrm{Fe}_{2}\left(\mathrm{C}_{5} \mathrm{H}_{5}\right)_{2}\left(\mathrm{C}_{24} \mathrm{H}_{25^{-}}\right.\right.$ $\left.\mathrm{O}_{4} \mathrm{P}\right)$ ], and the other phosphine sulfide is a purely planar-chiral compound (two planar chiral centres), i.e. bis[(2S $\left.S_{\mathrm{p}}\right)$-2-ethenylferrocen-1-yl]phenylphosphane sulfide, $\left[\mathrm{Fe}_{2}\left(\mathrm{C}_{5} \mathrm{H}_{5}\right)_{2}\left(\mathrm{C}_{20} \mathrm{H}_{17} \mathrm{PS}\right)\right]$. Owing to the stereocentres present, reactions performed on $\left[\left(\mathrm{Fc}^{\mathrm{A}}\right)_{2}(\mathrm{Ph}) \mathrm{P}\right]$-type compounds strongly favour one ferrocene unit over the other due to diastereoselectivity. Furthermore, we present four related structures where the ferrocene units are not identical $\left[\left(\mathrm{Fc}^{\mathrm{A}}\right)\left(\mathrm{Fc}^{\mathrm{B}}\right)(\mathrm{Ph}) \mathrm{P}\right]$. These are $\left\{\left(2 S_{\mathrm{p}}\right)-2-[(1 R)-1\right.$-(acetyloxy)ethyl]ferrocen-1-yl $\}\left[\left(2 S_{\mathrm{p}}\right)\right.$-2-ethenylferrocen-1yl]phenyl- $(S)$-phosphine sulfide, $\left[\mathrm{Fe}_{2}\left(\mathrm{C}_{5} \mathrm{H}_{5}\right)_{2}\left(\mathrm{C}_{22} \mathrm{H}_{21} \mathrm{O}_{2} \mathrm{PS}\right)\right]$, [ $\left(2 S_{\mathrm{p}}\right)$-2-ethenylferrocen-1-yl] $\left\{\left(2 S_{\mathrm{p}}\right)-2-[(1 R)\right.$-1-hydroxyethyl]ferrocen-1-yl $\}$ phenyl- $(S)$-phosphine sulfide, [ $\left.\mathrm{Fe}_{2}\left(\mathrm{C}_{5} \mathrm{H}_{5}\right)_{2}\left(\mathrm{C}_{20} \mathrm{H}_{19} \mathrm{OPS}\right)\right],\left\{\left(2 S_{\mathrm{p}}\right)-2-[(1 R)-1\right.$-(acetyloxy)ethyl]ferrocen-1yl $\}\left\{\left(2 S_{\mathrm{p}}\right)-2-[(1 R)-1\right.$-hydroxyethyl]ferrocen-1-yl $\}$ phenyl- $(R)$-phosphine sulfide, $\left[\mathrm{Fe}_{2}\left(\mathrm{C}_{5} \mathrm{H}_{5}\right)_{2}\left(\mathrm{C}_{22} \mathrm{H}_{23} \mathrm{O}_{3} \mathrm{PS}\right)\right]$, and $\left\{\left(2 S_{\mathrm{p}}\right)-2-[(1 R)-1\right.$-benzylamino)ethyl]ferrocen1-yl\}[(2S $\left.S_{\mathrm{p}}\right)$-2-ethenylferrocen-1-yl]phenyl- $(S)$-phosphine sulfide, $\left[\mathrm{Fe}_{2}\left(\mathrm{C}_{5} \mathrm{H}_{5}\right)_{2^{-}}\right.$ $\left.\left(\mathrm{C}_{27} \mathrm{H}_{26} \mathrm{NPS}\right)\right]$. All of the structures are accessible in one step from known precursors.

\section{Introduction}

Metallocenes decorated with at least two different substituents on the same ring are planar-chiral (Schaarschmidt \& Lang, 2013). They are useful as voluminous asymmetry-inducing groups in asymmetric transformations (Stepnicka, 2008). Even beyond academic research, disubstituted ferrocenes have been used in industrial asymmetric synthesis, for instance, in the hydrogenation of imines (Blaser et al., 2007).

The potential of asymmetric induction may be improved by designing ligands including two planar-chiral ferrocene units. One example is the phosphorous-linked diferrocene Pigiphos (Barbaro \& Togni, 1995), developed by the group of Togni. A multitude of ligands for transition-metal complexes have been synthesized and applied in asymmetric homogeneous catalysis [e.g. $\mathrm{Rh}^{\mathrm{I}}$ in hydrosilylations (Hayashi et al., 1974), $\mathrm{Rh}^{\mathrm{III}}$ in acetalizations (Barbaro et al., 1999), $\mathrm{Pd}^{\mathrm{II}}$ in hydroaminations (Gischig \& Togni, 2004, 2005), $\mathrm{Ru}^{\mathrm{II}}$ in transfer hydrogenations (Barbaro et al., 1997, 2003) and olefin cyclopropanations (Lee et al., 1999), and $\mathrm{Ni}^{\mathrm{II}}$ in hydroaminations (Fadini \& Togni, 2004, 2007, 2008), hydrophosphinations (Sadow \& Togni, 2005), Nazarov cyclizations (Walz \& Togni, 2008) and dipolar cycloadditons (Milosevic \& Togni, 2013)]. 
The performance of catalysts may be enhanced further by making the angle of the two ferrocene units rigid via ring closure, yielding a diferrocenyl (macro)cycle (Xiao et al., 2002), possibly with inclusion of another (planar-chiral) ferrocene unit (Wang et al., 2006). Stanphos (Broggini, 2003) is a $P, P$-ligand diferroceno ring and has been employed in asymmetric hydroalkoxylations (Barreiro et al., 2012) and the hydroxylation of 1,3-ketoesters (Smith et al., 2010).

In the course of the synthesis of cyclic diferrocene monophosphines with potential in asymmetric catalysis, we prepared compounds such as 6-9 (Fig. 1) potentially useful for a cyclization step. Their synthetic access starting from commercially available [1-(dimethylamino)ethyl]ferrocene (1) is outlined in Fig. 1. Beyond the desired cyclized products like 9a (Honegger \& Widhalm, 2019b), several side products were isolated and characterized.

\section{Experimental}

\subsection{Synthesis and crystallization}

2.1.1. Synthesis of 1,1-(phenylphosphanediyl)bis\{(2S)-2[(1R)-1-(acetyloxy)ethyl]ferrocene\} (7). Diaminophosphine 2 (619 mg, $1.00 \mathrm{mmol}$; Barreiro et al., 2012) was suspended in $\mathrm{Ac}_{2} \mathrm{O}(1 \mathrm{ml})$ in a flame-dried Schlenk tube under argon. The suspension was degassed and stirred for $7 \mathrm{~d}$ at room temperature, then for $7 \mathrm{~h}$ at $100{ }^{\circ} \mathrm{C}$. From the dark-red solution, $\mathrm{Ac}_{2} \mathrm{O}$ was removed under reduced pressure and the residue was purified by column chromatography $\left(\mathrm{SiO}_{2}, 0\right.$ $100 \%$ EtOAc in heptane) yielding diacetate 7 (yield $177 \mathrm{mg}$, $27 \%$ ) as pale-red crystals upon removal of the solvent (m.p. $\left.155-156{ }^{\circ} \mathrm{C}\right) .{ }^{1} \mathrm{H}$ NMR $\left(400 \mathrm{MHz}, \mathrm{CDCl}_{3}\right): \delta 7.62-7.56(m, 2 \mathrm{H})$, $7.37-7.30(m, 3 \mathrm{H}), 6.20(d t, J=2.5,6.4 \mathrm{~Hz}, 1 \mathrm{H}), 5.99(d t, J=2.9$, $6.4 \mathrm{~Hz}, 1 \mathrm{H}), 4.52(m, 1 \mathrm{H}), 4.45(m, 1 \mathrm{H}), 4.43(p t, J=2.5 \mathrm{~Hz}$, $1 \mathrm{H}), 4.35(\mathrm{~m}, 1 \mathrm{H}), 4.34(\mathrm{~m}, 1 \mathrm{H}), 4.30(m, 1 \mathrm{H}), 4.04(s, 5 \mathrm{H}), 3.72$ $(s, 5 \mathrm{H}), 2.11(s, 3 \mathrm{H}), 1.96(d, J=6.4 \mathrm{~Hz}, 3 \mathrm{H}), 1.56(d, J=6.4 \mathrm{~Hz}$, $3 \mathrm{H}), 1.32(s, 3 \mathrm{H}) .{ }^{31} \mathrm{P}$ NMR: $\delta-44.57(s)$. HRMS $(\mathrm{m} / z$ calculated for $\left.\mathrm{C}_{34} \mathrm{H}_{35} \mathrm{Fe}_{2} \mathrm{NaO}_{4} \mathrm{P}\right)[M+\mathrm{Na}]^{+}$673.0869; found 673.0877 .

2.1.2. Synthesis of monovinyl monoacetyl phosphine sulfide $8 \mathrm{a}$, monovinyl monohydroxy phosphine sulfide $8 \mathrm{~b}$ and monohydroxy monoacetyl phosphine sulfide 8c. Diamine phosphine sulfide 3 (653 mg, $1.00 \mathrm{mmol}$; Honegger \& Widhalm, 2019a) was suspended in $\mathrm{Ac}_{2} \mathrm{O}(1 \mathrm{ml})$ in a flamedried Schlenk tube. The suspension was degassed and stirred under argon for $7 \mathrm{~d}$ at room temperature, then for $7 \mathrm{~h}$ at $100{ }^{\circ} \mathrm{C}$ until the starting material was completely consumed (thin-layer chromatography, TLC). $\mathrm{Ac}_{2} \mathrm{O}$ was removed under reduced pressure and the residue was purified by column chromatography $\left(\mathrm{SiO}_{2}, 0-100 \%\right.$ EtOAc in heptane), yielding several mixed fractions, as well as compounds 8a (yield $135 \mathrm{mg}, 22 \%$ ), 8b (yield $99 \mathrm{mg}, 17 \%$ ) and $\mathbf{8 c}$ (yield $74 \mathrm{mg}$, $12 \%)$ as orange crystals upon removal of the solvent.

Analytical data for 8a: m.p. $177-178{ }^{\circ} \mathrm{C} .{ }^{1} \mathrm{H}$ NMR $\left(600 \mathrm{MHz}, \mathrm{CDCl}_{3}\right): \delta 8.13(d d, J=17.7,10.8 \mathrm{~Hz}, 1 \mathrm{H}), 7.77(d d$, $J=13.3,7.4 \mathrm{~Hz}, 2 \mathrm{H}), 7.47-7.41(m, 3 \mathrm{H}), 6.49(q, J=6.4 \mathrm{~Hz}$, $1 \mathrm{H}), 5.46(d d, J=17.7,1.7 \mathrm{~Hz}, 1 \mathrm{H}), 5.16(d d, J=10.8,1.6 \mathrm{~Hz}$,
1H), $4.84(m, 1 \mathrm{H}), 4.59(m, 1 \mathrm{H}), 4.36(s, 5 \mathrm{H}), 4.30(\mathrm{~m}, 1 \mathrm{H}), 4.28$ $(m, 1 \mathrm{H}), 4.14(s, 5 \mathrm{H}), 3.78(m, 1 \mathrm{H}), 3.54(m, 1 \mathrm{H}), 1.57(d, J=$ $6.5 \mathrm{~Hz}, 3 \mathrm{H}), 1.04(s, 3 \mathrm{H}) \cdot{ }^{13} \mathrm{C}\left\{{ }^{1} \mathrm{H}\right\}$ NMR: $\delta 169.39\left(\mathrm{C}_{\mathrm{q}}\right), 135.44$ $\left(d, J_{\mathrm{CP}}=88.3 \mathrm{~Hz}, \mathrm{C}_{\mathrm{q}}\right), 134.47(\mathrm{CH}), 132.29\left(d, J_{\mathrm{CP}}=10.5 \mathrm{~Hz}\right.$, $\mathrm{CH}), 130.77\left(d, J_{\mathrm{CP}}=2.8 \mathrm{~Hz}, \mathrm{CH}\right), 127.49\left(d, J_{\mathrm{CP}}=12.2 \mathrm{~Hz}\right.$, $\mathrm{CH}), 111.38\left(\mathrm{CH}_{2}\right), 88.95\left(d, J_{\mathrm{CP}}=12.1 \mathrm{~Hz}, \mathrm{C}_{\mathrm{q}}\right), 88.43\left(d, J_{\mathrm{CP}}=\right.$ $\left.12.0 \mathrm{~Hz}, \mathrm{C}_{\mathrm{q}}\right), 79.19\left(d, J_{\mathrm{CP}}=95.2 \mathrm{~Hz}, \mathrm{C}_{\mathrm{q}}\right), 75.84\left(d, J_{\mathrm{CP}}=\right.$ $11.4 \mathrm{~Hz}, \mathrm{CH}), 75.03\left(d, J_{\mathrm{CP}}=12.0 \mathrm{~Hz}, \mathrm{CH}\right), 74.46\left(d, J_{\mathrm{CP}}=\right.$ $\left.95.1 \mathrm{~Hz}, \mathrm{C}_{\mathrm{q}}\right), 71.15(\mathrm{CH}), 70.94(\mathrm{CH}), 70.60\left(d, J_{\mathrm{CP}}=9.0 \mathrm{~Hz}\right.$, $\mathrm{CH}), 69.88\left(d, J_{\mathrm{CP}}=10.2 \mathrm{~Hz}, \mathrm{CH}\right), 68.40\left(d, J_{\mathrm{CP}}=10.3 \mathrm{~Hz}\right.$, $\mathrm{CH}), 68.14\left(d, J_{\mathrm{CP}}=8.9 \mathrm{~Hz}, \mathrm{CH}\right), 67.99(\mathrm{CH}), 20.03\left(\mathrm{CH}_{3}\right)$, $18.49\left(\mathrm{CH}_{3}\right) \cdot{ }^{31} \mathrm{P}$ NMR: $\delta 39.14(\mathrm{~s})$. HRMS $(\mathrm{m} / \mathrm{z}$ calculated for $\left.\mathrm{C}_{32} \mathrm{H}_{31} \mathrm{Fe}_{2} \mathrm{O}_{2} \mathrm{PS}\right)[M]^{+}$622.0481, found 622.0462; $[M+\mathrm{Na}]^{+}$ 645.0379, found 645.0358; $[M+\mathrm{K}]^{+}$661.0118, found 661.0104.

Analytical data for 8b: m.p. $205-206^{\circ} \mathrm{C}$ (decomposition). ${ }^{1} \mathrm{H}$ $\operatorname{NMR}\left(600 \mathrm{MHz}, \mathrm{CDCl}_{3}\right): \delta 8.10(d d, J=17.6,10.8 \mathrm{~Hz}, 1 \mathrm{H})$, 7.87-7.81 (m, 2H), 7.51-7.42 (m, 3H), $5.49(d d, J=17.6,1.6 \mathrm{~Hz}$, $1 \mathrm{H}), 5.23-5.17(\mathrm{~m}, 1 \mathrm{H}), 5.20(d d, J=10.8,1.7 \mathrm{~Hz}, 1 \mathrm{H}), 4.88(m$, $1 \mathrm{H}), 4.49(\mathrm{~m}, 1 \mathrm{H}), 4.34(\mathrm{~s}, 5 \mathrm{H}), 4.33(\mathrm{~m}, 1 \mathrm{H}), 4.24(\mathrm{~m}, 1 \mathrm{H}), 4.17$ $(s, 5 \mathrm{H}), 3.77(m, 1 \mathrm{H}), 3.71(m, 1 \mathrm{H}), 2.41(d, J=5.3 \mathrm{~Hz}, 1 \mathrm{H})$, $1.26(d, J=6.6 \mathrm{~Hz}, 3 \mathrm{H}) \cdot{ }^{13} \mathrm{C}\left\{{ }^{1} \mathrm{H}\right\}$ NMR: $\delta 135.30\left(d, J_{\mathrm{CP}}=\right.$ $\left.87.1 \mathrm{~Hz}, \mathrm{C}_{\mathrm{q}}\right), 134.25(\mathrm{CH}), 132.10\left(d, J_{\mathrm{CP}}=10.3 \mathrm{~Hz}, \mathrm{CH}\right)$, $131.38\left(d, J_{\mathrm{CP}}=2.8 \mathrm{~Hz}, \mathrm{CH}\right), 127.96\left(d, J_{\mathrm{CP}}=12.1 \mathrm{~Hz}, \mathrm{CH}\right)$, $111.74\left(\mathrm{CH}_{2}\right), 94.90\left(d, J_{\mathrm{CP}}=12.3 \mathrm{~Hz}, \mathrm{C}_{\mathrm{q}}\right), 88.43\left(d, J_{\mathrm{CP}}=\right.$ $\left.11.8 \mathrm{~Hz}, \mathrm{C}_{\mathrm{q}}\right), 78.56\left(d, J_{\mathrm{CP}}=95.4 \mathrm{~Hz}, \mathrm{C}_{\mathrm{q}}\right), 75.05\left(d, J_{\mathrm{CP}}=\right.$ $12.0 \mathrm{~Hz}, \mathrm{CH}), 74.97\left(d, J_{\mathrm{CP}}=12.6 \mathrm{~Hz}, \mathrm{CH}\right), 73.48\left(d, J_{\mathrm{CP}}=\right.$ $\left.96.0 \mathrm{~Hz}, \mathrm{C}_{\mathrm{q}}\right), 71.22(\mathrm{CH}), 71.00\left(d, J_{\mathrm{CP}}=9.7 \mathrm{~Hz}, \mathrm{CH}\right), 70.72$ $(\mathrm{CH}), 70.10\left(d, J_{\mathrm{CP}}=10.4 \mathrm{~Hz}, \mathrm{CH}\right), 68.38\left(d, J_{\mathrm{CP}}=9.1 \mathrm{~Hz}\right.$, $\mathrm{CH}), 68.04\left(d, J_{\mathrm{CP}}=10.5 \mathrm{~Hz}, \mathrm{CH}\right), 64.38(\mathrm{CH}), 21.91\left(\mathrm{CH}_{3}\right)$. ${ }^{31} \mathrm{P}$ NMR: $\delta 40.52(\mathrm{~s})$. HRMS $\left(\mathrm{m} / z\right.$ calculated for $\mathrm{C}_{30} \mathrm{H}_{29} \mathrm{Fe}_{2}-$ OPS) $[M]^{+}$580.0376, found 580.0360; $[M+\mathrm{Na}]^{+}$603.0273, found 603.0273; $[M+\mathrm{K}]^{+}$619.0013, found 619.0018.

Analytical data for 8c: m.p. $174-175{ }^{\circ} \mathrm{C}$. ${ }^{1} \mathrm{H}$ NMR $\left(600 \mathrm{MHz}, \mathrm{CDCl}_{3}\right): \delta 8.20-8.14(m, 2 \mathrm{H}), 7.57-7.54(m, 3 \mathrm{H})$, $6.60(q, J=6.3 \mathrm{~Hz}, 1 \mathrm{H}), 4.93(m, 1 \mathrm{H}), 4.67(m, 2 \mathrm{H}), 4.49(m$, $1 \mathrm{H}), 4.38(\mathrm{~m}, 1 \mathrm{H}), 4.34(\mathrm{~m}, 1 \mathrm{H}), 4.11(s, 5 \mathrm{H}), 4.10(s, 5 \mathrm{H}), 1.90$ $(s, 3 \mathrm{H}), 1.83(d, J=6.3 \mathrm{~Hz}, 3 \mathrm{H}), 1.63(m, 1 \mathrm{H}), 1.43(d d, J=19.2$, $6.4 \mathrm{~Hz}, 1 \mathrm{H}), 1.40(d, J=6.6 \mathrm{~Hz}, 3 \mathrm{H}) \cdot{ }^{13} \mathrm{C}\left\{{ }^{1} \mathrm{H}\right\}$ NMR: $\delta 169.70$ $\left(\mathrm{C}_{\mathrm{q}}\right), 135.42\left(d, J_{\mathrm{CP}}=87.3 \mathrm{~Hz}, \mathrm{C}_{\mathrm{q}}\right), 132.21\left(d, J_{\mathrm{CP}}=10.5 \mathrm{~Hz}\right.$, $\mathrm{CH}), 131.47\left(d, J_{\mathrm{CP}}=2.9 \mathrm{~Hz}, \mathrm{CH}\right), 127.88\left(d, J_{\mathrm{CP}}=12.2 \mathrm{~Hz}\right.$, $\mathrm{CH}), 93.49\left(d, J_{\mathrm{CP}}=11.8 \mathrm{~Hz}, \mathrm{C}_{\mathrm{q}}\right), 93.47\left(d, J_{\mathrm{CP}}=13.5 \mathrm{~Hz}, \mathrm{C}_{\mathrm{q}}\right)$, $75.85\left(d, J_{\mathrm{CP}}=13.4 \mathrm{~Hz}, \mathrm{CH}\right), 74.53\left(\mathrm{C}_{\mathrm{q}}\right), 73.91\left(\mathrm{C}_{\mathrm{q}}\right), 71.53(d$, $\left.J_{\mathrm{CP}}=9.3 \mathrm{~Hz}, \mathrm{CH}\right), 70.93(\mathrm{CH}), 70.60\left(d, J_{\mathrm{CP}}=60.4 \mathrm{~Hz}, \mathrm{CH}\right)$, $70.58(\mathrm{CH}), 70.27\left(d, J_{\mathrm{CP}}=9.3 \mathrm{~Hz}, \mathrm{CH}\right), 69.48\left(d, J_{\mathrm{CP}}=\right.$ $10.1 \mathrm{~Hz}, \mathrm{CH}), 68.87(\mathrm{CH}), 68.56\left(d, J_{\mathrm{CP}}=10.9 \mathrm{~Hz}, \mathrm{CH}\right), 64.20$ $(\mathrm{CH}), 22.37\left(\mathrm{CH}_{3}\right), 22.23\left(\mathrm{CH}_{3}\right), 21.80\left(\mathrm{CH}_{3}\right) .{ }^{31} \mathrm{P}$ NMR: $\delta$ $39.12(s)$. HRMS $\left(m / z\right.$ calculated for $\left.\mathrm{C}_{32} \mathrm{H}_{33} \mathrm{Fe}_{2} \mathrm{O}_{3} \mathrm{PS}\right)[M]^{+}$ 640.0587 , found $640.0566 ;[M+\mathrm{Na}]^{+} 663.0485$, found 663.0463 .

2.1.3. Catalytic experiments: asymmetric allylic alkylation (Widhalm et al., 1996). In a flame-dried Schlenk tube, the diferrocene ligand $(0.010 \mathrm{mmol}, 1 \mathrm{~mol} \%)$ and $[\mathrm{Pd}(\text { allyl }) \mathrm{Cl}]_{2}$ (1.8 mg, $0.005 \mathrm{mmol}, 0.5 \mathrm{~mol} \%$ ) were dissolved in degassed DCM (1 ml) in that order under argon. The yellow solution was stirred for 20 min while it turned orange. To the solution, freshly distilled 1,3-diphenylallyl acetate $(252 \mathrm{mg}, 1.00 \mathrm{mmol})$, dimethyl malonate $(0.340 \mathrm{ml}, 3.00 \mathrm{mmol}, 3$ equiv. $)$, bis(trimethylsilyl)acetamide $(0.740 \mathrm{ml}, 3.00 \mathrm{mmol}, 3$ equiv. $)$ and a 


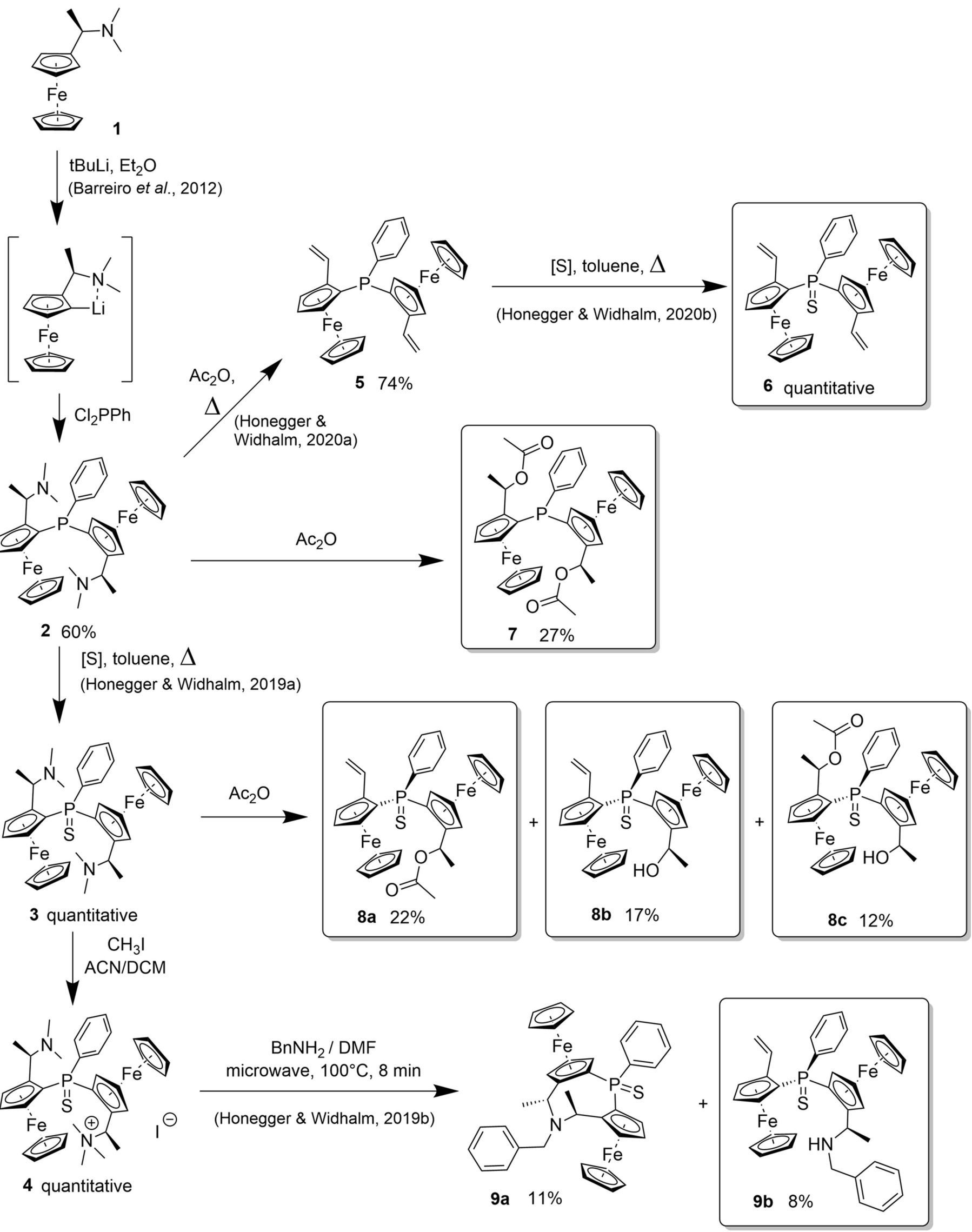

Figure 1

Synthetic route towards the crystallized phosphorous-linked diferrocenes $6,7,8 \mathbf{a}, 8 \mathbf{b}, 8 \mathbf{c}$ and $9 \mathbf{b}$. 
Table 1

Experimental details.

6

Crystal data

Chemical formula

$M_{\mathrm{r}}$

Crystal system, space group

Temperature (K)

$a, b, c(\AA)$

$\alpha, \beta, \gamma\left(^{\circ}\right)$

$V\left(\AA^{3}\right)$

$Z$

Radiation type

$\mu\left(\mathrm{mm}^{-1}\right)$

Crystal size (mm)

Data collection

Diffractometer

Absorption correction

$T_{\min }, T_{\max }$

No. of measured, independent and observed $[I>2 \sigma(I)]$ reflections

$R_{\text {int }}$

$(\sin \theta / \lambda)_{\max }\left(\AA^{-1}\right)$

Refinement

$R\left[F^{2}>2 \sigma\left(F^{2}\right)\right], w R\left(F^{2}\right), S$

No. of reflections

No. of parameters

No. of restraints

$\mathrm{H}$-atom treatment

$\Delta \rho_{\max }, \Delta \rho_{\min }\left(\mathrm{e} \AA^{-3}\right)$

Absolute structure

Absolute structure parameter
$\left[\mathrm{Fe}_{2}\left(\mathrm{C}_{5} \mathrm{H}_{5}\right)_{2}\left(\mathrm{C}_{20} \mathrm{H}_{17} \mathrm{PS}\right)\right]$

562.24

Monoclinic, $P 2_{1}$

130

8.4709 (7), 14.1401 (11),

$21.0310(17)$

90, $91.880(3), 90$

2517.7 (4)

Mo $K \alpha$

1.32

$0.28 \times 0.25 \times 0.13$

Bruker X8 APEXII

Multi-scan (SADABS; Bruker, 2008

$0.620,0.746$

$35484,14468,13352$

0.038

0.708

$0.032,0.071,1.03$

14468

613

1

$\mathrm{H}$-atom parameters constrained

$0.42,-0.37$

Flack $x$ determined using 5751 quotients $\left[\left(I^{+}\right)-\left(I^{-}\right)\right] /$

$\left[\left(I^{+}\right)+\left(I^{-}\right)\right]$(Parsons et al., 2013) $-0.019(5)$
7

$\left[\mathrm{Fe}_{2}\left(\mathrm{C}_{5} \mathrm{H}_{5}\right)_{2}\left(\mathrm{C}_{24} \mathrm{H}_{25} \mathrm{O}_{4} \mathrm{P}\right)\right]$

650.29

Orthorhombic, $P 2_{1} 2_{1} 2_{1}$

130

7.631 (2), 10.877 (2), 36.025 (8)

$90,90,90$

2990.2 (12)

Mo $K \alpha$

1.06

$0.15 \times 0.08 \times 0.06$

$8 \mathbf{a}$

Multi-scan (SADABS; Bruker, 2008)

$0.562,0.745$

$46099,5441,4085$

0.150

0.602

$0.065,0.122,1.04$

5441

374

0

$\mathrm{H}$-atom parameters constrained $0.94,-0.43$

Flack $x$ determined using 1235 quotients $\left[\left(I^{+}\right)-\left(I^{-}\right)\right] /$

$\left[\left(I^{+}\right)+\left(I^{-}\right)\right]$(Parsons et al., 2013) $-0.02(2)$
$\left[\mathrm{Fe}_{2}\left(\mathrm{C}_{5} \mathrm{H}_{5}\right)_{2}\left(\mathrm{C}_{22} \mathrm{H}_{21} \mathrm{O}_{2} \mathrm{PS}\right)\right]$

622.30

Orthorhombic, $P 2_{1} 2_{1} 2_{1}$

100

7.4923 (3), 12.0133 (4), 31.758 (1)

$90,90,90$

2858.45 (17)

Mo $K \alpha$

1.17

$0.21 \times 0.14 \times 0.05$

Bruker D8 Venture

Multi-scan ( $S A D A B S$; Bruker, 2012)

$0.607,0.746$

52739, 8330, 7214

0.062

0.704

$0.032,0.062,1.04$

8330

345

0

$\mathrm{H}$-atom parameters constrained

$0.34,-0.34$

Flack $x$ determined using 2809 quotients $\left[\left(I^{+}\right)-\left(I^{-}\right)\right] /$

$\left[\left(I^{+}\right)+\left(I^{-}\right)\right]$(Parsons et al., 2013) $-0.007(5)$
Crystal data

Chemical formula

$M_{\text {r }}$

Crystal system, space group

Temperature (K)

$a, b, c(\AA)$

$\alpha, \beta, \gamma\left({ }^{\circ}\right)$

$V\left(\AA^{3}\right)$

Z

Radiation type

$\mu\left(\mathrm{mm}^{-1}\right)$

Crystal size (mm)

Data collection

Diffractometer

Absorption correction

$T_{\min }, T_{\max }$

No. of measured, independent and observed $[I>2 \sigma(I)]$ reflections

$R_{\text {int }}$

$(\sin \theta / \lambda)_{\max }\left(\AA^{-1}\right)$

$\left[\mathrm{Fe}_{2}\left(\mathrm{C}_{5} \mathrm{H}_{5}\right)_{2}\left(\mathrm{C}_{20} \mathrm{H}_{19} \mathrm{OPS}\right)\right]$

580.26

Orthorhombic, $P 2_{1} 2_{1} 2_{1}$

130

7.5285 (3), 17.6463 (7)

$39.3333(15)$

90, 90,90

$5225.4(4)$

8

Mo $K \alpha$

1.27

$0.25 \times 0.2 \times 0.17$

$\left[\mathrm{Fe}_{2}\left(\mathrm{C}_{5} \mathrm{H}_{5}\right)_{2}\left(\mathrm{C}_{22} \mathrm{H}_{23} \mathrm{O}_{3} \mathrm{PS}\right)\right]$

640.31

Orthorhombic, $P 2_{1} 2_{1} 2_{1}$

130

7.8204 (11), 17.835 (3), 20.394 (2)

$90,90,90$

$2844.5(7)$

4

Mo $K \alpha$

1.18

$0.1 \times 0.06 \times 0.01$

Bruker X8 APEXII

Multi-scan (SADABS; Bruker, 2008)

$0.568,0.745$

$35430,5258,3417$

0.167

0.606

$0.054,0.093,1.02$

5258

356

0

635

$\mathrm{H}$-atom parameters constrained
$\left[\mathrm{Fe}_{2}\left(\mathrm{C}_{5} \mathrm{H}_{5}\right)_{2}\left(\mathrm{C}_{27} \mathrm{H}_{26} \mathrm{NPS}\right)\right]$

669.40

Orthorhombic, $P 2_{1} 2_{1} 2_{1}$

130

12.3578 (9), 14.4342 (10),

$17.4796(15)$

90, 90, 90

3117.9 (4)

4

Mo $K \alpha$

1.08

$0.22 \times 0.11 \times 0.09$

Bruker APEXII CCD

Multi-scan (SADABS; Bruker, 2008)

$0.486,0.746$

38997, 9160, 7062

0.075

0.706

$0.040,0.091,0.98$

9160

384

0

$\mathrm{H}$ atoms treated by a mixture of independent and constrained refinement 
Table 1 (continued)

\begin{tabular}{llll}
\hline & $\mathbf{8 b}$ & $\mathbf{8 c}$ & 9b \\
\hline$\Delta \rho_{\max }, \Delta \rho_{\min }\left(\mathrm{e} \AA^{-3}\right)$ & $2.08,-1.80$ & $0.42,-0.41$ & $0.43,-0.35$ \\
Absolute structure & Flack $x$ determined using 4140 & Flack $x$ determined using 1051 & Flack $x$ determined using 2549 \\
& quotients $\left[\left(I^{+}\right)-\left(I^{-}\right)\right] /$ & quotients $\left[\left(I^{+}\right)-\left(I^{-}\right)\right] /$ & quotients $\left[\left(I^{+}\right)-\left(I^{-}\right)\right] /$ \\
Absolute structure parameter & {$\left[\left(I^{+}\right)+\left(I^{-}\right)\right]($Parsons et al., 2013) } & {$\left[\left(I^{+}\right)+\left(I^{-}\right)\right]($Parsons et al., 2013) } & {$\left[\left(I^{+}\right)+\left(I^{-}\right)\right]($Parsons et al., 2013) } \\
& $-0.010(7)$ & $0.00(2)$ & $-0.016(10)$
\end{tabular}

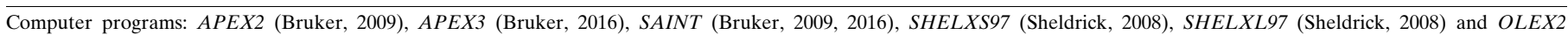
(Dolomanov et al., 2009).

catalytic amount of potassium acetate were added in that order. The reaction mixture was degassed once and stirred for $48 \mathrm{~h}$ at room temperature until the catalytic conversion was complete. To the solution, $\mathrm{Et}_{2} \mathrm{O}(15 \mathrm{ml})$ was added. The organic layer was washed twice with saturated aqueous $\mathrm{NH}_{4} \mathrm{Cl}$ solution, dried over $\mathrm{Na}_{2} \mathrm{SO}_{4}$ and the solvent removed under reduced pressure. The residue was dried, dissolved in DCM $(2 \mathrm{ml})$ and filtered through $\mathrm{SiO}_{2}$. The enantiomeric excess (e.e.) was detected via chiral high-performance liquid chromatography (HPLC; Chiralcel OD-H, 2\% isopropanol in $n$-heptane).

\subsection{Melting points}

The melting points were measured on a Reichelt Thermovar Kofler apparatus and are uncorrected.

\subsection{Chiral high-performance liquid chromatography (HPLC)}

HPLC analysis was performed on an Agilent Technologies 1200 series system using a Chiralcel OD-H chiral column.

\subsection{NMR spectroscopy}

Routine NMR spectra were recorded on a $400 \mathrm{MHz}$ Bruker AVIII 400 spectrometer operating at $400.27\left({ }^{1} \mathrm{H}\right), 100.66\left({ }^{13} \mathrm{C}\right)$ and $162.04 \mathrm{MHz}\left({ }^{31} \mathrm{P}\right)$ with an autosampler. The ${ }^{1} \mathrm{H}$ and ${ }^{13} \mathrm{C}\left\{{ }^{1} \mathrm{H}\right\}$ NMR spectra used for substance characterization were recorded either on a $600 \mathrm{MHz}$ Bruker AVIII 600 spectrometer operating at $600.25\left({ }^{1} \mathrm{H}\right)$ and $150.95 \mathrm{MHz}\left({ }^{13} \mathrm{C}\right)$ or on a Bruker AVIII 700 spectrometer operating at $700.40\left({ }^{1} \mathrm{H}\right)$ and $176.13 \mathrm{MHz}\left({ }^{13} \mathrm{C}\right) .{ }^{13} \mathrm{C}$ NMR spectra were recorded in
J-modulated mode. NMR chemical shifts are referenced to nondeuterated $\mathrm{CHCl}_{3}$ residual shifts at $7.26 \mathrm{ppm}$ for ${ }^{1} \mathrm{H} \mathrm{NMR}$ and to $\mathrm{CDCl}_{3}$ at $77.00 \mathrm{ppm}$ for ${ }^{13} \mathrm{C} \mathrm{NMR}$. Coupling patterns in the ${ }^{1} \mathrm{H}$ and ${ }^{13} \mathrm{C}$ NMR spectra are denoted using standard abbreviations: $s$ (singlet), $d$ (doublet), $t$ (triplet), $q$ (quartet), $m$ (multiplet) and $p$ (pseudo). For the ${ }^{13} \mathrm{C}$ NMR spectra, carbon resonances were identified as $\mathrm{C}_{\mathrm{q}}, \mathrm{CH}, \mathrm{CH}_{2}$ and $\mathrm{CH}_{3}$.

\subsection{High-resolution mass spectroscopy (HRMS)}

HRMS were recorded by a Bruker Maxis ESI oa-RTOF mass spectrometer equipped with a quadrupole analyzer ion guide.

\subsection{Preparative column chromatography}

Preparative column chromatography was carried out on an Biotage Isolera One automated flash chromatography instrument using self-packed columns containing either $\mathrm{SiO}_{2}$ (Macherey-Nagel silica gel $60 \mathrm{M}$, particle size $40-63 \mu \mathrm{m}$ ) or $\mathrm{Al}_{2} \mathrm{O}_{3}$ (Merck aluminium oxide 90 standardized, activation grade II-III).

\subsection{X-ray diffractometry}

X-ray diffraction was performed on a Bruker X8 APEXII diffractometer, a Bruker D8 Venture diffractometer and a Bruker APEXII CCD diffractometer, all with Mo $K \alpha$ radiation.

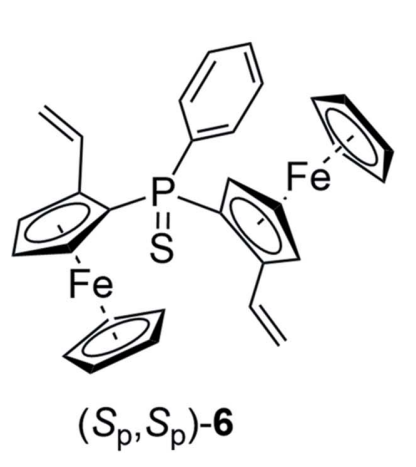

(a)

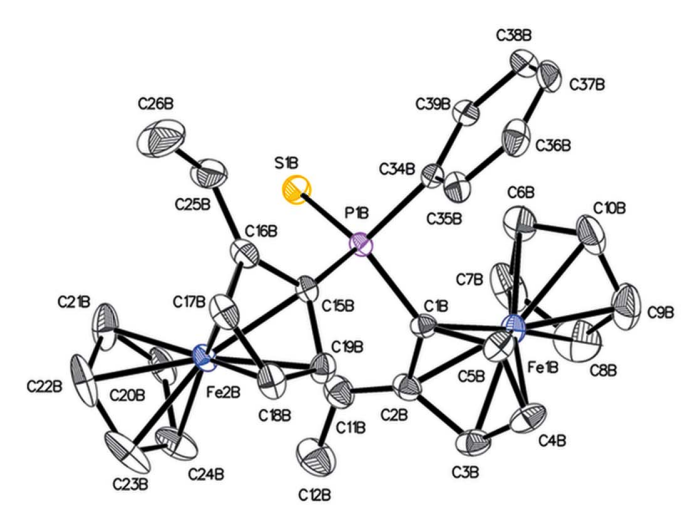

(b)

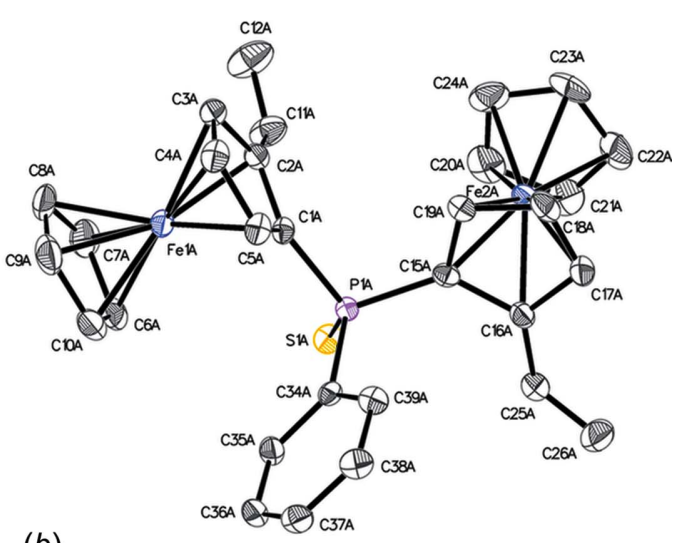

Figure 2

(a) Chemical structure and (b) displacement ellipsoid plot of divinyl 6. The ellipsoid probability level of this figure and all subsequent figures is $50 \%$. 


\subsection{Refinement}

The structures were solved by direct methods and refined using full-matrix least-squares techniques. Non-H atoms were refined with anisotropic displacement parameters. $\mathrm{H}$ atoms were inserted at calculated positions and refined using a riding model. $\mathrm{C}-\mathrm{H}$ bond lengths in the aromatic and olefin bond systems were constrained at $0.950 \AA$, aliphatic $\mathrm{CH}_{2}$ groups at $0.990 \AA$ and aliphatic $\mathrm{CH}_{3}$ groups at $0.980 \AA$. The default values of SHELXL (Sheldrick, 2008) were used for the ridingatom model. Fixed $U_{\text {iso }}$ values of 1.2 times were used for all $\mathrm{C}(\mathrm{H})$ and $\mathrm{C}(\mathrm{H}, \mathrm{H})$ groups, and fixed $U_{\text {iso }}$ values of 1.5 times were used for all $\mathrm{C}(\mathrm{H}, \mathrm{H}, \mathrm{H})$ and $\mathrm{O}(\mathrm{H})$ groups. Details for each compound are summarized in the CIF file under the keyword '_refine_special_details'.

The position of the acidic atom $\mathrm{H} 1 B$ at $\mathbf{8 b}$ was stabilized using a length-fixing restraint. Several reflections, primarily inner ones, have been omitted to avoid wrong interpretations.

The amine $\mathrm{H}$ atom of compound $\mathbf{9 b}$ was refined taking account of the two possible configurations of the $\mathrm{N}$ atom. The

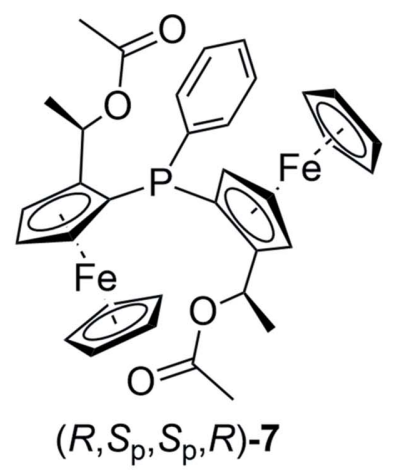

(a)

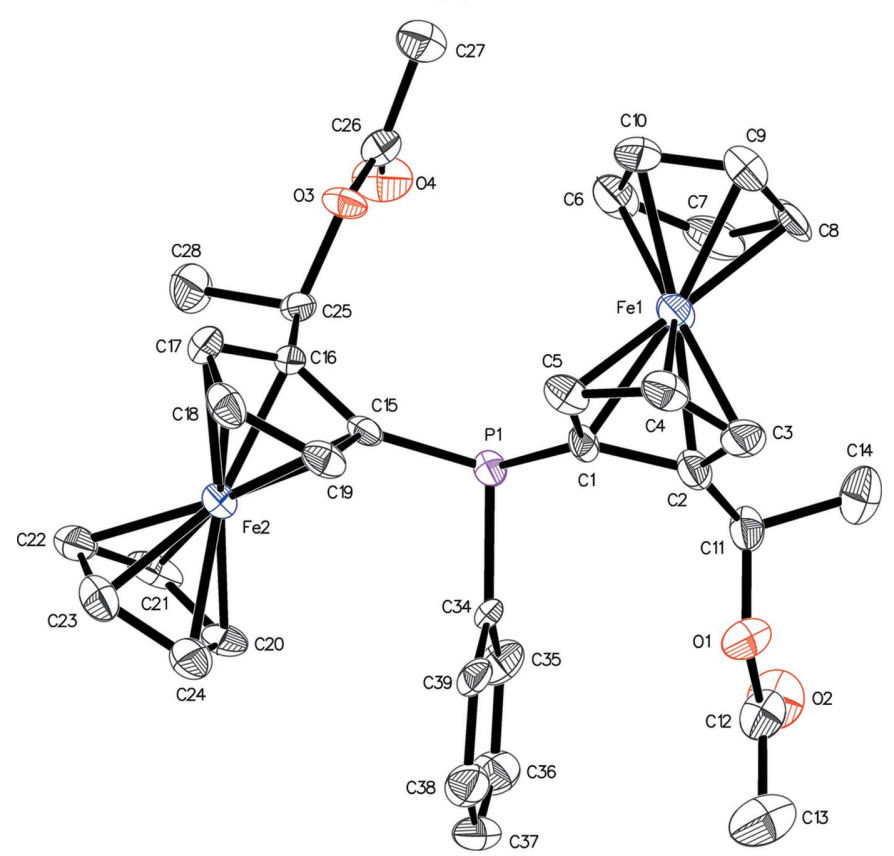

(b)

Figure 3

(a) Chemical structure and (b) displacement ellipsoid plot of diacetate 7.

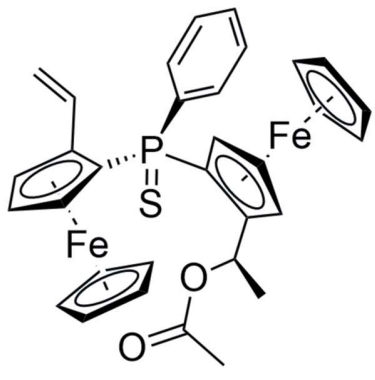

$$
\left(S_{\mathrm{p}}, S, S_{\mathrm{p}}, R\right)-\mathbf{8} \mathbf{a}
$$

(a)

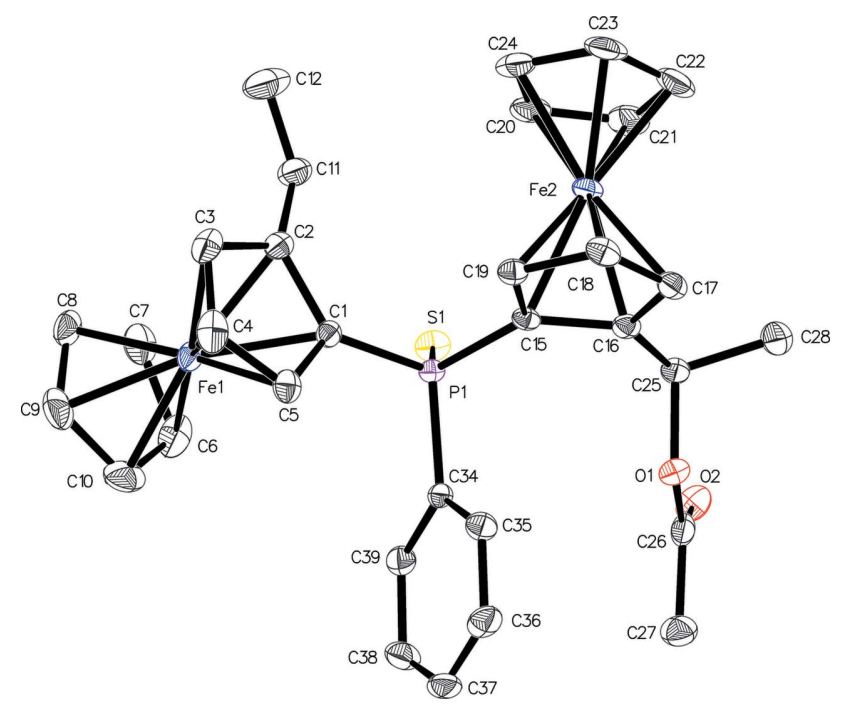

Figure 4

(b)

(a) Chemical structure and (b) displacement ellipsoid plot of monoacetate 8a.

choice was stable and in agreement with the position of available electron density.

Crystal data, data collection and structure refinement details are summarized in Table 1.

\section{Results and discussion}

The syntheses carried out in the framework of this study are summarized in Fig. 1. The structure of the central diferrocene precursor 2 has been deposited previously (Steiner \& Pioda, 1999) in the Cambridge Structural Database (Groom et al., 2016). First, we eliminated the dimethylamine groups of 2 to obtain divinyl structure $\left(S_{\mathrm{p}}, S_{\mathrm{p}}\right)-\mathbf{5}$ by heating in acetic anhydride according to Honegger \& Widhalm (2020). The sensitive phosphine was then protected by reaction with elemental sulfur to quantitatively produce divinylphosphine sulfide $\left(S_{\mathrm{p}}, S_{\mathrm{p}}\right)$-6, shown in Fig. 2 (Honegger et al., 2020). The substance was readily isolated as orange crystals upon removal of the solvent. Cyclization attempts of $\mathbf{6}$ via ring-closing metathesis (RCM) failed, possibly due to the separation of the vinyl $\mathrm{C}$ atoms, steric strain in the product or interference of the Grubbs catalyst with the phosphine sulfide. However, Lewis acid-catalyzed hydrovinylation afforded the desired allcarbon backbone (Honegger \& Widhalm, 2020). 


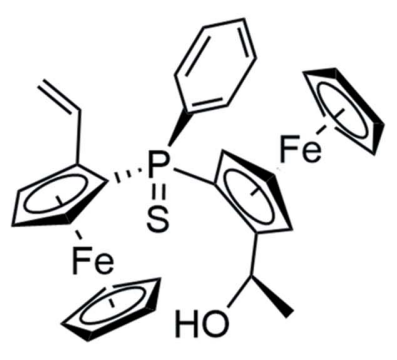

$\left(S_{p}, S, S_{p}, R\right)-8 b$

(a)

Figure 5

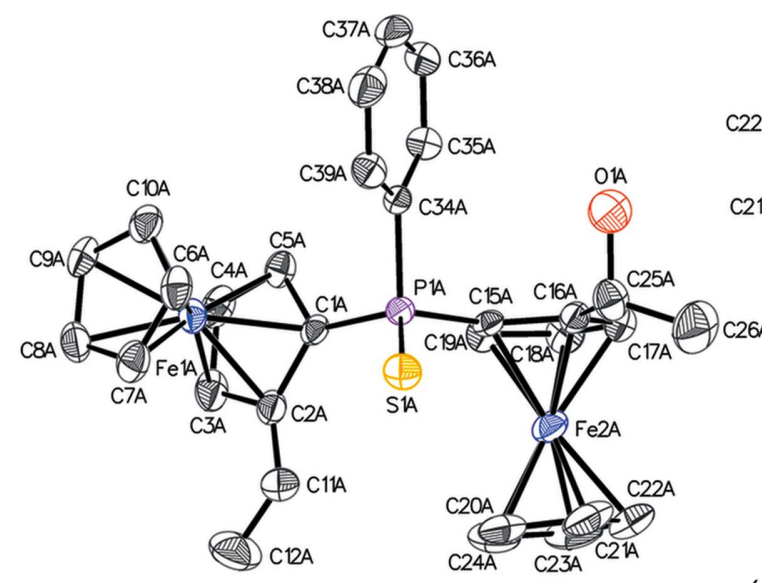

(b)

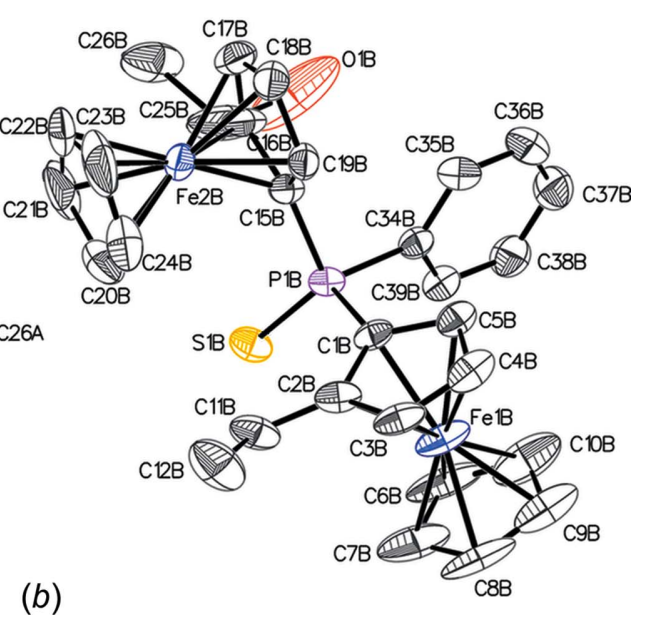

(a) Chemical structure and (b) displacement ellipsoid plot of monohydroxide $\mathbf{8 b}$.

For an alternative approach, we replaced the diamino groups with more capable leaving groups in order to close the ring with bidentate nucleophiles. In one of these attempts, we replaced the amines by acetate groups using acetic anhydride. The resulting diacetate $\left(R, S_{\mathrm{p}}, S_{\mathrm{p}}, R\right)-\mathbf{7}$ crystallized upon removal of the solvent (Fig. 3). Alternatively, the reactive phosphino group was protected by reaction with elemental sulfur to yield phosphine sulfide $\left(R, S_{\mathrm{p}}, S_{\mathrm{p}}, R\right)$-3 (Honegger \& Widhalm, 2019a). In contrast to the unprotected phosphine, we could not obtain the diacetate from derivative $\left(R, S_{\mathrm{p}}, S_{\mathrm{p}}, R\right)$ $\mathbf{3}$, but from the reaction mixture, three compounds, namely, $\left(S_{\mathrm{p}}, S, S_{\mathrm{p}}, R\right)-\mathbf{8 a} \quad$ (Fig. 4), $\quad\left(S_{\mathrm{p}}, S, S_{\mathrm{p}}, R\right)-\mathbf{8 b} \quad($ Fig. $\quad 5) \quad$ and $\left(R, S_{\mathrm{p}}, R, S_{\mathrm{p}}, R\right)$-8c (Fig. 6) with acetate, hydroxy or vinyl side groups instead, could be isolated, indicating that the substitution was followed by elimination or cleavage of the acetyl group.

Alternatively, an attempt was made to convert the diamine $\left(R, S_{\mathrm{p}}, S_{\mathrm{p}}, R\right)-3$ into a diammonium salt, yet only monomethiodide 4 was obtained in quantitative yield. Bridging with benzylamine afforded ring-closed 9a along with the monoeliminated crystalline side product $\left(S_{\mathrm{p}}, S, S_{\mathrm{p}}, R\right)$-9b (Fig. 7), both in poor yield (12 and $13 \%$, respectively) (Honegger \& Widhalm, 2019b).

In the symmetric diferrocenes $\left(S_{\mathrm{p}}, S_{\mathrm{p}}\right)-\mathbf{6}$ and $\left(S_{\mathrm{p}}, S_{\mathrm{p}}\right)-\mathbf{7}$, the ferrocene subunits are identical. Fig. 8 shows a system developed to distinguish them into an $r e$-site and an $s i$-site. Taking divinyl compound $\left(S_{\mathrm{p}}, S_{\mathrm{p}}\right)-\mathbf{6}$ as an example, both ferrocene units are planar-chiral $\left(S_{\mathrm{p}}\right)$. The $\mathrm{P}$ atom in compound $\left(S_{\mathrm{p}}, S_{\mathrm{p}}\right)-\mathbf{6}$ is prochiral; if any of the two vinylferrocene groups are modified, the ferrocene substituents become distinguishable and the $\mathrm{P}$ atom thus a chiral centre. Fig. 9 shows a hypothetical Markovnikov regioselective addition of $\mathrm{HNu}$ to divinyl $\left(S_{\mathrm{p}}, S_{\mathrm{p}}\right)-\mathbf{6}$, Nu standing for a generalized nucleophile. Depending on whether $\mathrm{HNu}$ is added to the re-site or si-site vinyl group, the $\mathrm{P}$ atom becomes an $(S)$ - or $(R)$-chiral centre, respectively. The two possible products are diastereomers since the reaction turns the P-atom centre from prochiral to centre-chiral. In addition, this reaction introduces a new chiral centre, but in a diastereoselective fashion since one of the two sites is blocked by the other ring of the ferrocenyl unit
(Marquarding et al., 1970). The two possible products are diastereomers, differing only in the resulting configuration of

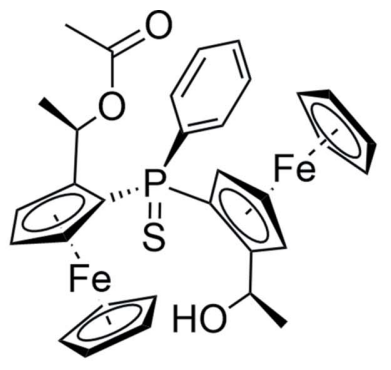

$\left(R, S_{\mathrm{p}}, R, S_{\mathrm{p}}, R\right)-8 \mathrm{c}$

(a)

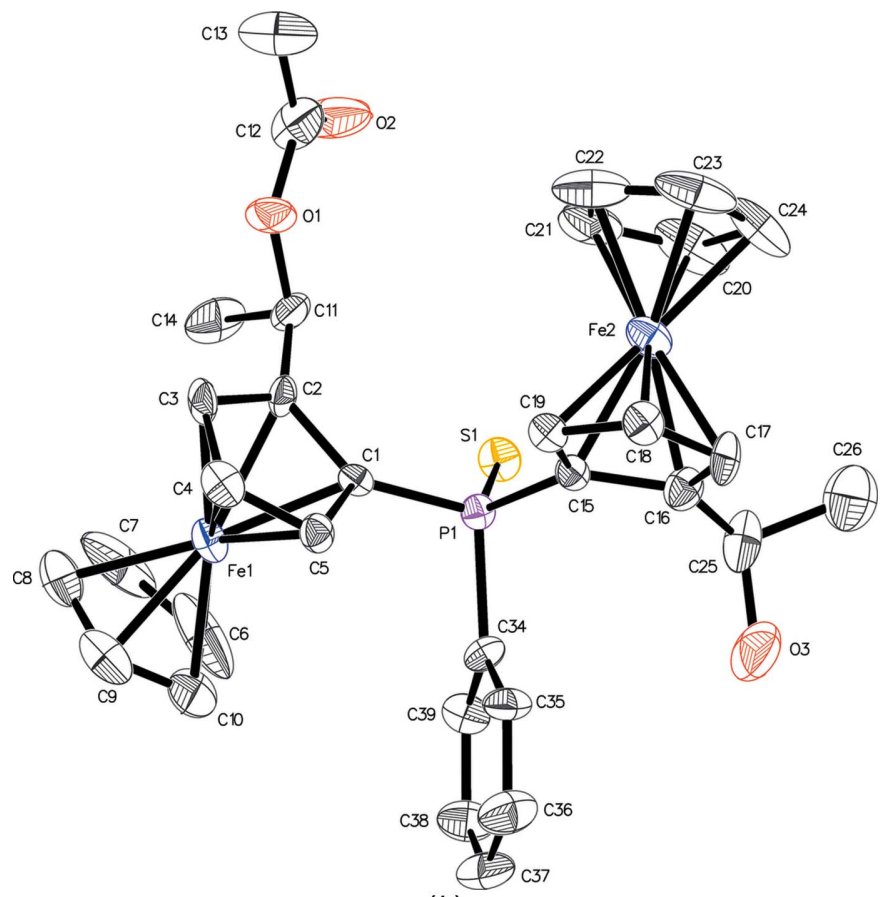

Figure 6

(b)

(a) Chemical structure and (b) displacement ellipsoid plot of monoacetatemonohydroxide $\mathbf{8 c}$. 


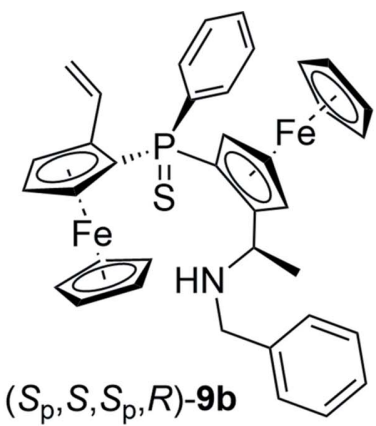

(a)

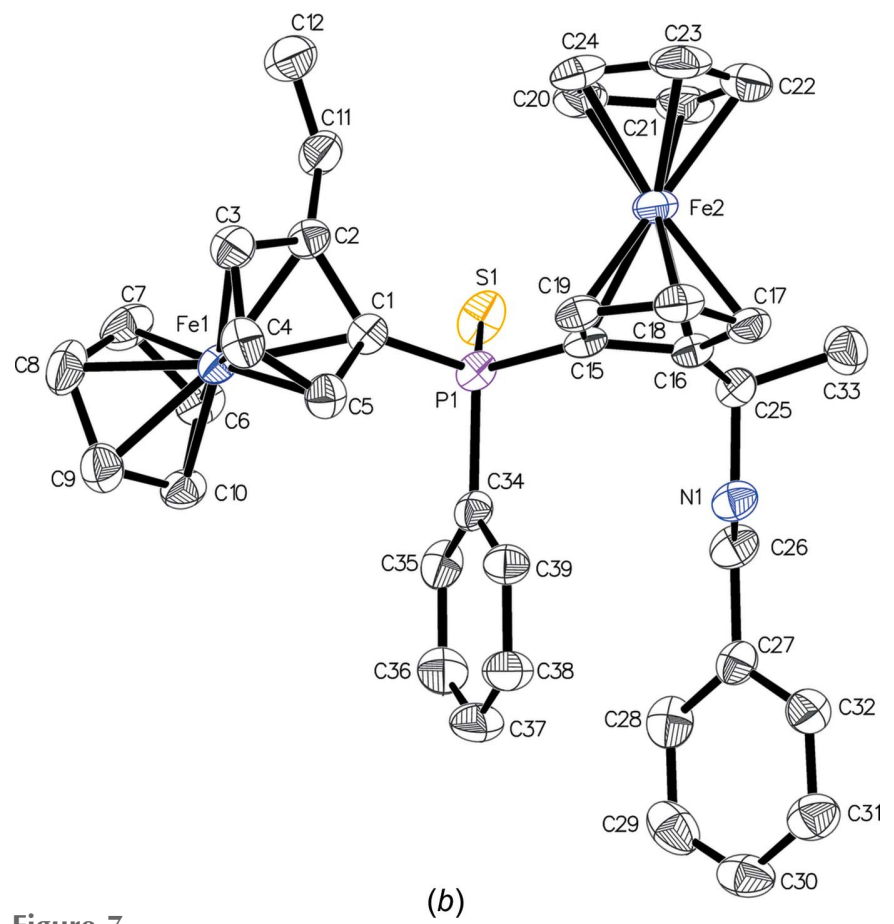

Figure 7

(a) Chemical structure and (b) displacement ellipsoid plot of benzylamine $9 b$.

the $\mathrm{P}$ atom (epimers). In the compounds presented in this article, we know the configuration at the $\left(S_{\mathrm{p}}\right)$-disubstituted ferrocene will be selectively $(R)$, since the approach of the nucleophile from the $(S)$-site is blocked by the other cyclopentadienyl (Cp) ring (Marquarding et al., 1970). Fig. 9 illustrates this by rotating the two possible products by $180^{\circ}$ for better comparison with the other product; again, only the configuration of the $\mathrm{P}$ atom differs. We only observed the formation of one of the two possible diastereomers, hence the reaction proceeds diastereoselectively, as was observed for different reactions throughout this study. Typically, the re-site of the symmetrical $\left(S_{\mathrm{p}}, S_{\mathrm{p}}\right)$-precursors was more reactive.

Thus, the vinylferrocene groups are diastereotopic and their respective NMR shifts can be distinguished despite their apparent equality in connectivity when neglecting stereochemical aspects. In fact, protons attached to the inner vinyl protons in $\left(S_{\mathrm{p}}, S_{\mathrm{p}}\right)-\mathbf{6}$ differ so greatly in chemical shift that one of the two is found at a higher field than even aromatic protons ( $\delta=8.1$ ppm; Honegger et al., 2020).

The preferred conformation of ferrocenyl units with both $\mathrm{Cp}$ rings is a perpendicular arrangement, with the reactive $r e$ ferrocene closer to the small sulfur residue and the less reactive $s i$-ferrocene shielded by the bulkier phenyl group.

For the asymmetric diferrocene compounds $\left(S_{\mathrm{p}}, S, S_{\mathrm{p}}, R\right)-\mathbf{8 a}$, $\left(S_{\mathrm{p}}, S, S_{\mathrm{p}}, R\right)-\mathbf{8 b}$ and $\left(S_{\mathrm{p}}, S, S_{\mathrm{p}}, R\right)-\mathbf{9 b}$, the ferrocenyl at the smaller sulfur group bears the more bulky substituent (acetate, hydroxy and benzyl), while the other ferrocenyl unit at the larger phenyl ring is substituted with a sterically less demanding vinyl group. Only hydroxyacetate $\left(S_{\mathrm{p}}, R, S_{\mathrm{p}}, R\right)$-8c shows the opposite preference. The preferred geometry might be mainly controlled by subtle inter- and intramolecular steric interactions as no $\pi-\pi$ interactions could be detected. The protic $\mathrm{H}$ atoms in compounds $8 \mathbf{b}, 8 \mathbf{c}(\mathrm{O}-\mathrm{H}$ group) and $\mathbf{9 b}(\mathrm{N}-\mathrm{H}$ group) form intramolecular hydrogen bonds with the $\pi$-system of the P-substituted phenyl group.

Since we obtained the sufficiently stable compound $\left(S_{\mathrm{p}}, S_{\mathrm{p}}\right)$ $\mathbf{6}$ in large enough quantities, we tested its asymmetry-inducing performance as a ligand in an in-situ formed $\mathrm{Pd}^{\mathrm{II}}$ complex used for asymmetric allylic alkylation according to Widhalm et al. (1996). This purely planar-chiral compound achieved an enantiomeric excess of $35 \%$, which is less than what we found for previously known $\left(R, S_{\mathrm{p}}, S_{\mathrm{p}}, R\right)-\mathbf{2}(57 \%)$. Regardless, the coordination structure of $\left(S_{\mathrm{p}}, S_{\mathrm{p}}\right)-\mathbf{6}$ and $\mathrm{Pd}^{\mathrm{II}}$ remains an interesting question since neither phosphine $\left(S_{\mathrm{p}}, S_{\mathrm{p}}\right)-\mathbf{5}$ nor its phosphine oxide analog were able to activate $\mathrm{Pd}^{\mathrm{II}}$. We could not isolate the catalytically active $\mathrm{Pd}^{\mathrm{II}}$ complex to study its structure, but we speculate that the phosphine sulfide might act as an electron donor to form a dative bond in transitionmetal catalysts.

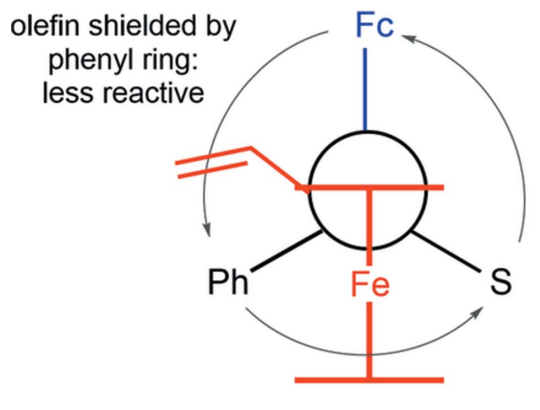

si-ferrocene

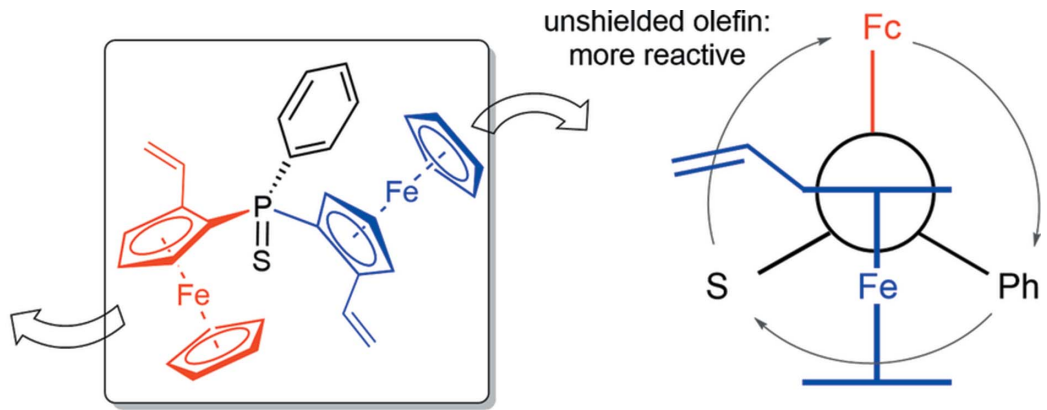

re-ferrocene

Figure 8

The re/si nomenclature for diferrocenes developed in the framework of this study to distinguish between the two ferrocenyl subunits. 


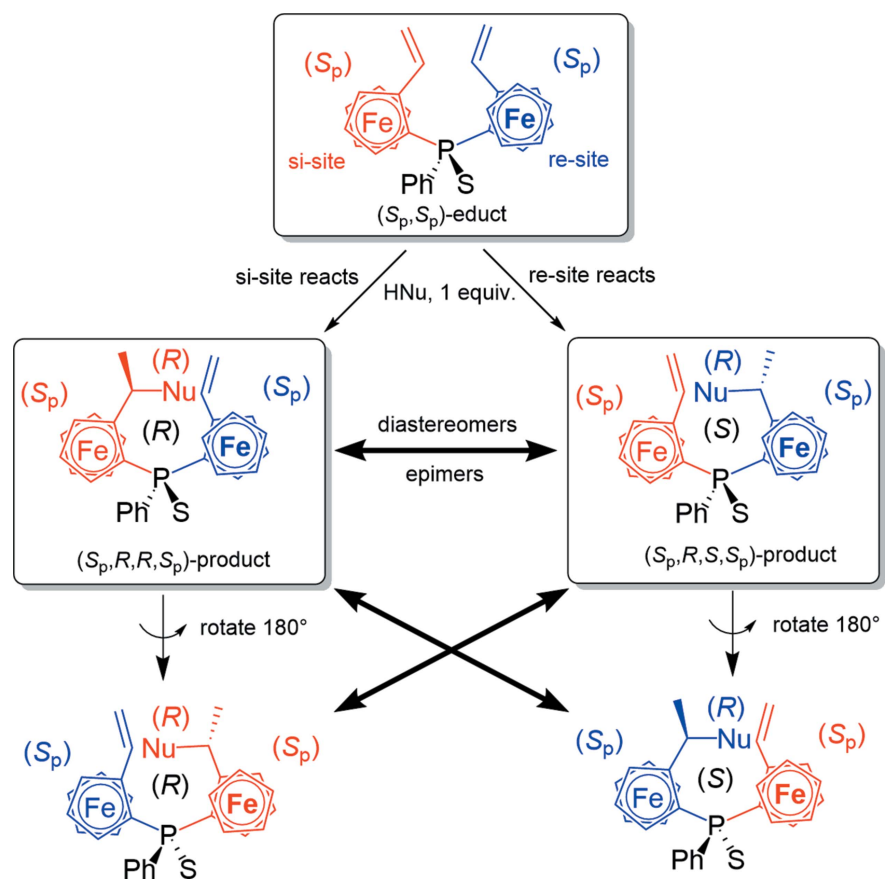

Figure 9

Hypothetical Markovnikov-selective addition of $\mathrm{HNu}$ to a symmetrical di(vinylferrocene), with $\mathrm{Nu}$ standing for an arbitrary nucleophile. The other Cp rings have been omitted for clarity and represented indirectly by the planar-chiral configuration $\left(S_{\mathrm{p}}\right)$.

\section{Conclusion}

We present the crystal structures of six homochiral phosphorous-linked diferrocenes. All the ferrocene units are planarchiral $\left(S_{\mathrm{p}}\right)$ and five of the compounds include one or two centre-chiral $\mathrm{C}$ atoms $(R)$ also. Interestingly, the molecules lack strong intermolecular interactions and exhibit no $\pi$-stacking, even though most of the $\mathrm{C}$ atoms are aromatic. Compounds $8 \mathbf{b}, 8 \mathbf{c}$ and $9 \mathbf{b}$ include acidic $\mathrm{H}$ atoms $(R \mathrm{O}-\mathrm{H}$ and $R_{2} \mathrm{~N}-\mathrm{H}$ ) capable of forming hydrogen bridges with the $\pi$-electron systems of the phenyl ring.

Four of the presented compounds contain two differently substituted ferrocene units, $\left[\left(\mathrm{Fc}^{\mathrm{A}}\right)\left(\mathrm{Fc}^{\mathrm{B}}\right)(\mathrm{Ph}) \mathrm{P}\right]$, while in the other two compounds, the two ferrocene compounds are equal, $\left[\left(\mathrm{Fc}^{\mathrm{A}}\right)_{2}(\mathrm{Ph}) \mathrm{P}\right]$. Due to the planar chirality of the ferrocene units, the linking $\mathrm{P}$ atom is prochiral and one of the two equal ferrocene units reacts far more readily with reagents than the other due to diastereoselectivity. This ability of selective chemical ferrocene subunit differentiation suggests the application of such diferrocenes in asymmetric organic chemistry, for instance, as ligands, catalysts and auxiliaries.

\section{References}

Barbaro, P., Bianchini, C., Giambastiani, G. \& Togni, A. (2003). Eur. J. Inorg. Chem. 2003, 4166-4172.
Barbaro, P., Bianchini, C., Oberhauser, W. \& Togni, A. (1999). J. Mol. Catal. A Chem. 145, 139-146.

Barbaro, P., Bianchini, C. \& Togni, A. (1997). Organometallics, 16, 3004-3014.

Barbaro, P. \& Togni, A. (1995). Organometallics, 14, 3570-3573.

Barreiro, E., Broggini, D., Adrio, L., White, A., Schwenk, R., Togni, A. \& Hii, K. (2012). Organometallics, 31, 3745-3754.

Blaser, H.-U., Pugin, B., Spindler, F. \& Thommen, M. (2007). Acc. Chem. Res. 40, 1240-1250.

Broggini, D. (2003). PhD thesis, Swiss Federal Institute of Technology Zurich, Switzerland.

Bruker (2008). SADABS. Bruker AXS Inc., Madison, Wisconsin, USA.

Bruker (2009). APEX2 and SAINT. Bruker AXS Inc., Madison, Wisconsin, USA.

Bruker (2012). SADABS. Bruker AXS Inc., Madison, Wisconsin, USA.

Bruker (2016). APEX3 and SAINT. Bruker AXS Inc., Madison, Wisconsin, USA.

Dolomanov, O. V., Bourhis, L. J., Gildea, R. J., Howard, J. A. K. \& Puschmann, H. (2009). J. Appl. Cryst. 42, 339-341.

Fadini, L. \& Togni, A. (2004). CHIMIA Int. J. Chem. 58, 208-211.

Fadini, L. \& Togni, A. (2007). Helv. Chim. Acta, 90, 411-424.

Fadini, L. \& Togni, A. (2008). Tetrahedron Asymmetry, 19, 2555-2562.

Gischig, S. \& Togni, A. (2004). Organometallics, 23, 2479-2487.

Gischig, S. \& Togni, A. (2005). Eur. J. Inorg. Chem. 2005, 47454754.

Groom, C. R., Bruno, I. J., Lightfoot, M. P. \& Ward, S. C. (2016). Acta Cryst. B72, 171-179.

Hayashi, T., Yamamoto, K. \& Kumada, M. (1974). Tetrahedron Lett. 15, 4405-4408.

Honegger, P., Scheibelberger, L. \& Widhalm, M. (2020). Molbank, 2020, M1130.

Honegger, P. \& Widhalm, M. (2019a). Molbank, 2019, M1090.

Honegger, P. \& Widhalm, M. (2019b). Molbank, 2019, M1098.

Honegger, P. \& Widhalm, M. (2020). Molbank, 2020, M1105.

Lee, H. M., Bianchini, C., Jia, G. \& Barbaro, P. (1999). Organometallics, 18, 1961-1966.

Marquarding, D., Klusacek, H., Gokel, G., Hoffmann, P. \& Ugi, I. (1970). J. Am. Chem. Soc. 92, 5389-5393.

Milosevic, S. \& Togni, A. (2013). J. Org. Chem. 78, 9638-9646.

Parsons, S., Flack, H. D. \& Wagner, T. (2013). Acta Cryst. B69, 249 259.

Sadow, A. D. \& Togni, A. (2005). J. Am. Chem. Soc. 127, 1701217024.

Schaarschmidt, D. \& Lang, H. (2013). Organometallics, 32, 56685704.

Sheldrick, G. M. (2008). Acta Cryst. A64, 112-122.

Smith, A. M. R., Rzepa, H. S., White, A. J. P., Billen, D. \& Hii, K. K. (2010). J. Org. Chem. 75, 3085-3096.

Steiner, I. \& Pioda, G. (1999). Private communication (refcode QUKKIQ; CCDC deposition number 134133). CCDC, Cambridge, UK.

Stepnicka, P. (2008). In Ferrocenes: Ligands, Materials and Biomolecules. Chichester: John Wiley \& Sons.

Walz, I. \& Togni, A. (2008). Chem. Commun. pp. 4315-4317.

Wang, Y., Weissensteiner, W., Mereiter, K. \& Spindler, F. (2006). Helv. Chim. Acta, 89, 1772-1782.

Widhalm, M., Wimmer, P. \& Klintschar, G. (1996). J. Organomet. Chem. 523, 167-178.

Xiao, L., Weissensteiner, W., Mereiter, K. \& Widhalm, M. (2002). J. Org. Chem. 67, 2206-2214. 


\section{supporting information}

Acta Cryst. (2021). C77, 152-160 [https://doi.org/10.1107/S2053229621001996]

\section{Synthesis and characterization of enantiopure planar-chiral phosphorus-linked diferrocenes}

\section{Philipp Honegger, Alexander Roller and Michael Widhalm}

Computing details

Data collection: APEX2 (Bruker, 2009) for (6), (7), (8b), (8c), (9b); APEX3 (Bruker, 2016) for (8a). Cell refinement: SAINT (Bruker, 2009) for (6), (7), (8b), (8c), (9b); SAINT (Bruker, 2016) for (8a). Data reduction: SAINT (Bruker, 2009) for (6), (7), (8b), (8c), (9b); SAINT (Bruker, 2016) for (8a). For all structures, program(s) used to solve structure: SHELXS97 (Sheldrick, 2008); program(s) used to refine structure: SHELXL97 (Sheldrick, 2008); molecular graphics: OLEX2 (Dolomanov et al., 2009); software used to prepare material for publication: OLEX2 (Dolomanov et al., 2009).

$\operatorname{Bis}\left[\left(2 S_{\mathrm{p}}\right)\right.$-2-ethenylferrocen-1-yl] phenylphosphane sulfide (6)

Crystal data

$\left[\mathrm{Fe}_{2}\left(\mathrm{C}_{5} \mathrm{H}_{5}\right)_{2}\left(\mathrm{C}_{20} \mathrm{H}_{17} \mathrm{PS}\right)\right]$

$M_{r}=562.24$

Monoclinic, $P 2_{1}$ $a=8.4709$ (7) $\AA$

$b=14.1401(11) \AA$

$c=21.0310(17) \AA$

$\beta=91.880(3)^{\circ}$

$V=2517.7(4) \AA^{3}$

$Z=4$

$F(000)=1160$

$D_{\mathrm{x}}=1.483 \mathrm{Mg} \mathrm{m}^{-3}$

Mo $K \alpha$ radiation, $\lambda=0.71073 \AA$

Cell parameters from 9832 reflections

$\theta=2.4-29.6^{\circ}$

$\mu=1.32 \mathrm{~mm}^{-1}$

$T=130 \mathrm{~K}$

Block, clear brown

$0.28 \times 0.25 \times 0.13 \mathrm{~mm}$

Data collection

Bruker X8 APEXII diffractometer

Radiation source: sealed xray tube, Incoatec IuS

Detector resolution: 8 pixels $\mathrm{mm}^{-1}$

$\varphi$ and $\omega$ scans

Absorption correction: multi-scan

(SADABS; Bruker, 2008

$T_{\min }=0.620, T_{\max }=0.746$

35484 measured reflections

14468 independent reflections

13352 reflections with $I>2 \sigma(I)$

$R_{\text {int }}=0.038$

$\theta_{\max }=30.2^{\circ}, \theta_{\min }=1.9^{\circ}$

$h=-11 \rightarrow 11$

$k=-18 \rightarrow 19$

$l=-29 \rightarrow 25$

Refinement

Refinement on $F^{2}$

Least-squares matrix: full

$R\left[F^{2}>2 \sigma\left(F^{2}\right)\right]=0.032$

$w R\left(F^{2}\right)=0.071$

$S=1.03$

14468 reflections

613 parameters

1 restraint

Primary atom site location: structure-invariant direct methods

Hydrogen site location: inferred from neighbouring sites

$\mathrm{H}$-atom parameters constrained

$w=1 /\left[\sigma^{2}\left(F_{\mathrm{o}}^{2}\right)+(0.0334 P)^{2}+0.1684 P\right]$

where $P=\left(F_{\mathrm{o}}^{2}+2 F_{\mathrm{c}}^{2}\right) / 3$

$(\Delta / \sigma)_{\max }=0.003$

$\Delta \rho_{\max }=0.42 \mathrm{e} \AA^{-3}$ 
$\Delta \rho_{\min }=-0.37 \mathrm{e} \AA^{-3}$

Special details

Geometry. All esds (except the esd in the dihedral angle between two 1.s. planes) are estimated using the full covariance matrix. The cell esds are taken into account individually in the estimation of esds in distances, angles and torsion angles; correlations between esds in cell parameters are only used when they are defined by crystal symmetry. An approximate (isotropic) treatment of cell esds is used for estimating esds involving l.s. planes.

Refinement._olex2_refinement_description

1. Fixed Uiso At 1.2 times of: All $\mathrm{C}(\mathrm{H})$ groups, $\mathrm{All} \mathrm{C}(\mathrm{H}, \mathrm{H})$ groups 2.a Aromatic/amide $\mathrm{H}$ refined with riding coordinates: C3A(H3A), C4A(H4A), C5A(H5A), C6A(H6A), C7A(H7A), C8A(H8A), C9A(H9A), C10A(H10A), C11A(H11A), C17A(H17A), C18A(H18A), C19A(H19A), C20A(H20A), C21A(H21A), C22A(H22A), C23A(H23A), C24A(H24A), C25A(H25A), C35A(H35A), C36A(H36A), C37A(H37A), C38A(H38A), C39A(H39A), C3B(H3B), C4B(H4B), C5B(H5B), C6B(H6B), C7B(H7B), C8B(H8B), C9B(H9B), C10B(H10B), C11B(H11B), C17B(H17B), C18B(H18B), C19B(H19B), C20B(H20B), C21B(H21B), C22B(H22B), C23B(H23B), C24B(H24B), C25B(H25B), C35B(H35B), $\mathrm{C} 36 \mathrm{~B}(\mathrm{H} 36 \mathrm{~B}), \mathrm{C} 37 \mathrm{~B}(\mathrm{H} 37 \mathrm{~B}), \mathrm{C} 38 \mathrm{~B}(\mathrm{H} 38 \mathrm{~B}), \mathrm{C} 39 \mathrm{~B}(\mathrm{H} 39 \mathrm{~B}) 2 . \mathrm{b} \mathrm{X}=\mathrm{CH} 2$ refined with riding coordinates: C12A(H12A,H12B), C26A(H26A,H26B), C12B(H12C,H12D), C26B(H26C,H26D)

Fractional atomic coordinates and isotropic or equivalent isotropic displacement parameters $\left(\AA^{2}\right)$

\begin{tabular}{|c|c|c|c|c|}
\hline & $x$ & $y$ & $z$ & $U_{\text {iso }} * / U_{\text {eq }}$ \\
\hline Fe1A & $0.59127(5)$ & $0.38914(3)$ & $0.76817(2)$ & $0.01812(9)$ \\
\hline $\mathrm{Fe} 2 \mathrm{~A}$ & $0.34181(5)$ & $0.28622(3)$ & $1.02977(2)$ & $0.02048(10)$ \\
\hline S1A & $0.19010(8)$ & $0.24467(5)$ & $0.83624(4)$ & $0.02041(15)$ \\
\hline P1A & $0.28675(8)$ & $0.35995(5)$ & $0.87163(3)$ & $0.01445(14)$ \\
\hline $\mathrm{C} 1 \mathrm{~A}$ & 0.4891 & $0.3803(2)$ & $0.85297(13)$ & $0.0169(5)$ \\
\hline $\mathrm{C} 2 \mathrm{~A}$ & $0.6190(3)$ & $0.3136(2)$ & 0.85085 (14) & $0.0200(6)$ \\
\hline $\mathrm{C} 3 \mathrm{~A}$ & 0.7578 & $0.3677(2)$ & $0.83929(14)$ & $0.0219(6)$ \\
\hline $\mathrm{H} 3 \mathrm{~A}$ & 0.860707 & 0.342545 & 0.834320 & $0.026^{*}$ \\
\hline $\mathrm{C} 4 \mathrm{~A}$ & $0.7172(4)$ & $0.4648(2)$ & $0.83646(15)$ & $0.0238(6)$ \\
\hline $\mathrm{H} 4 \mathrm{~A}$ & 0.788282 & 0.515584 & 0.830075 & $0.029 *$ \\
\hline C5A & 0.5525 & $0.4732(2)$ & $0.84477(14)$ & $0.0212(6)$ \\
\hline $\mathrm{H} 5 \mathrm{~A}$ & 0.494171 & 0.530619 & 0.844890 & $0.025 *$ \\
\hline C6A & $0.4324(4)$ & $0.3460(3)$ & $0.69950(15)$ & $0.0273(7)$ \\
\hline H6A & 0.329694 & 0.321977 & 0.706505 & $0.033^{*}$ \\
\hline C7A & $0.5717(4)$ & 0.2919 & $0.69722(16)$ & $0.0351(8)$ \\
\hline H7A & 0.579373 & 0.225308 & 0.702281 & $0.042 *$ \\
\hline C8A & $0.6980(4)$ & 0.3544 & $0.68606(17)$ & $0.0400(9)$ \\
\hline H8A & 0.805586 & 0.336929 & 0.682343 & $0.048 *$ \\
\hline C9A & $0.6370(5)$ & 0.4470 & $0.68138(17)$ & $0.0385(9)$ \\
\hline H9A & 0.696106 & 0.502846 & 0.674043 & $0.046^{*}$ \\
\hline $\mathrm{C} 10 \mathrm{~A}$ & $0.4714(4)$ & $0.4421(3)$ & $0.68958(16)$ & $0.0315(8)$ \\
\hline H10A & 0.399862 & 0.493815 & 0.688593 & $0.038 *$ \\
\hline C11A & $0.6117(4)$ & $0.2110(2)$ & $0.85674(19)$ & $0.0317(8)$ \\
\hline H11A & 0.510176 & 0.182481 & 0.857177 & $0.038 *$ \\
\hline $\mathrm{C} 12 \mathrm{~A}$ & $0.7362(5)$ & 0.1550 & $0.8615(2)$ & $0.0439(10)$ \\
\hline $\mathrm{H} 12 \mathrm{~A}$ & 0.839555 & 0.181071 & 0.861221 & $0.053 *$ \\
\hline H12B & 0.722528 & 0.088529 & 0.865200 & $0.053 *$ \\
\hline
\end{tabular}




\begin{tabular}{|c|c|c|c|c|}
\hline $\mathrm{C} 15 \mathrm{~A}$ & $0.2891(3)$ & $0.37435(19)$ & $0.95609(13)$ & $0.0165(5)$ \\
\hline $\mathrm{C} 16 \mathrm{~A}$ & $0.1574(3)$ & $0.3685(2)$ & $0.99792(14)$ & $0.0199(6)$ \\
\hline $\mathrm{C} 17 \mathrm{~A}$ & $0.2133(4)$ & $0.3990(2)$ & $1.05901(15)$ & $0.0251(6)$ \\
\hline H17A & 0.152217 & 0.401675 & 1.096047 & $0.030^{*}$ \\
\hline $\mathrm{C} 18 \mathrm{~A}$ & $0.3743(4)$ & 0.4249 (2) & $1.05595(15)$ & $0.0253(7)$ \\
\hline $\mathrm{H} 18 \mathrm{~A}$ & 0.439104 & 0.447892 & 1.090285 & $0.030^{*}$ \\
\hline C19A & 0.4219 (4) & $0.4105(2)$ & $0.99281(14)$ & $0.0200(6)$ \\
\hline H19A & 0.524061 & 0.422635 & 0.977344 & $0.024 *$ \\
\hline $\mathrm{C} 20 \mathrm{~A}$ & $0.3700(5)$ & $0.1500(2)$ & 0.99818 (19) & $0.0363(8)$ \\
\hline $\mathrm{H} 20 \mathrm{~A}$ & 0.354740 & 0.128376 & 0.955649 & $0.044 *$ \\
\hline $\mathrm{C} 21 \mathrm{~A}$ & $0.2537(5)$ & $0.1534(3)$ & $1.0451(2)$ & $0.0370(9)$ \\
\hline $\mathrm{H} 21 \mathrm{~A}$ & 0.146630 & 0.134393 & 1.039531 & $0.044 *$ \\
\hline $\mathrm{C} 22 \mathrm{~A}$ & $0.3241(5)$ & 0.1897 (3) & 1.10095 (19) & $0.0392(9)$ \\
\hline $\mathrm{H} 22 \mathrm{~A}$ & 0.272511 & 0.199723 & 1.139823 & $0.047^{*}$ \\
\hline $\mathrm{C} 23 \mathrm{~A}$ & $0.4849(5)$ & $0.2092(3)$ & $1.08999(19)$ & $0.0387(9)$ \\
\hline $\mathrm{H} 23 \mathrm{~A}$ & 0.560390 & 0.234164 & 1.119883 & $0.046^{*}$ \\
\hline $\mathrm{C} 24 \mathrm{~A}$ & $0.5121(5)$ & $0.1842(3)$ & 1.0259 (2) & $0.0384(9)$ \\
\hline $\mathrm{H} 24 \mathrm{~A}$ & 0.609726 & 0.189669 & 1.005267 & $0.046^{*}$ \\
\hline $\mathrm{C} 25 \mathrm{~A}$ & $-0.0024(4)$ & $0.3364(3)$ & $0.98061(15)$ & $0.0268(7)$ \\
\hline $\mathrm{H} 25 \mathrm{~A}$ & -0.015263 & 0.298115 & 0.943691 & $0.032 *$ \\
\hline $\mathrm{C} 26 \mathrm{~A}$ & $-0.1297(4)$ & $0.3564(3)$ & $1.01206(18)$ & $0.0425(10)$ \\
\hline $\mathrm{H} 26 \mathrm{~A}$ & -0.121857 & 0.394507 & 1.049228 & $0.051^{*}$ \\
\hline $\mathrm{H} 26 \mathrm{~B}$ & -0.229525 & 0.332743 & 0.997623 & $0.051^{*}$ \\
\hline C34A & $0.1858(3)$ & $0.4661(2)$ & $0.84285(13)$ & $0.0167(5)$ \\
\hline $\mathrm{C} 35 \mathrm{~A}$ & $0.1176(3)$ & $0.4667(2)$ & $0.78168(14)$ & $0.0208(6)$ \\
\hline $\mathrm{H} 35 \mathrm{~A}$ & 0.118585 & 0.411088 & 0.756380 & $0.025^{*}$ \\
\hline $\mathrm{C} 36 \mathrm{~A}$ & $0.0481(4)$ & $0.5487(2)$ & $0.75779(15)$ & $0.0247(6)$ \\
\hline H36A & 0.003166 & 0.549417 & 0.715804 & $0.030^{*}$ \\
\hline C37A & $0.0440(4)$ & $0.6296(2)$ & $0.79492(16)$ & $0.0255(7)$ \\
\hline H37A & -0.002851 & 0.685774 & 0.778184 & $0.031^{*}$ \\
\hline $\mathrm{C} 38 \mathrm{~A}$ & 0.1075 (4) & $0.6288(2)$ & $0.85581(16)$ & $0.0258(7)$ \\
\hline H38A & 0.102374 & 0.684046 & 0.881348 & $0.031 *$ \\
\hline C39A & $0.1794(4)$ & $0.5473(2)$ & $0.88043(16)$ & $0.0221(6)$ \\
\hline H39A & 0.223789 & 0.547136 & 0.922511 & $0.027^{*}$ \\
\hline Fe1B & $0.53902(5)$ & $0.23164(3)$ & $0.47208(2)$ & $0.01884(9)$ \\
\hline $\mathrm{Fe} 2 \mathrm{~B}$ & $0.80931(5)$ & $0.47524(3)$ & $0.26413(2)$ & $0.01774(9)$ \\
\hline S1B & $0.95491(9)$ & $0.23114(5)$ & $0.36024(3)$ & $0.02119(15)$ \\
\hline P1B & $0.85530(8)$ & $0.33963(5)$ & $0.40045(3)$ & $0.01426(14)$ \\
\hline C1B & $0.6510(3)$ & $0.32641(19)$ & $0.41800(14)$ & $0.0164(5)$ \\
\hline $\mathrm{C} 2 \mathrm{~B}$ & $0.5245(3)$ & $0.2811(2)$ & $0.38130(14)$ & 0.0217 (6) \\
\hline C3B & $0.3818(4)$ & $0.3004(2)$ & $0.41331(16)$ & $0.0266(7)$ \\
\hline H3B & 0.279689 & 0.278405 & 0.400389 & $0.032 *$ \\
\hline C4B & $0.4162(4)$ & $0.3570(2)$ & $0.46686(18)$ & $0.0277(7)$ \\
\hline H4B & 0.341367 & 0.380487 & 0.495600 & $0.033^{*}$ \\
\hline C5B & $0.5810(4)$ & $0.3730(2)$ & $0.47076(16)$ & $0.0234(6)$ \\
\hline H5B & 0.635955 & 0.408555 & 0.502793 & $0.028^{*}$ \\
\hline C6B & $0.6873(5)$ & $0.1251(2)$ & $0.50028(17)$ & $0.0333(8)$ \\
\hline H6B & 0.793577 & 0.117307 & 0.488182 & $0.040^{*}$ \\
\hline
\end{tabular}




\begin{tabular}{|c|c|c|c|c|}
\hline C7B & $0.5564(6)$ & $0.0883(3)$ & $0.46780(19)$ & $0.0437(11)$ \\
\hline H7B & 0.555952 & 0.051390 & 0.430039 & $0.052 *$ \\
\hline $\mathrm{C} 8 \mathrm{~B}$ & $0.4207(5)$ & $0.1171(3)$ & $0.5027(2)$ & $0.0516(12)$ \\
\hline H8B & 0.313519 & 0.102389 & 0.492333 & $0.062 *$ \\
\hline C9B & $0.4764(5)$ & $0.1711(3)$ & $0.5551(2)$ & $0.0450(10)$ \\
\hline H9B & 0.412882 & 0.199608 & 0.586226 & $0.054^{*}$ \\
\hline $\mathrm{C} 10 \mathrm{~B}$ & $0.6396(5)$ & $0.1755(3)$ & $0.55361(17)$ & $0.0375(9)$ \\
\hline H10B & 0.707315 & 0.207273 & 0.583520 & $0.045^{*}$ \\
\hline C11B & 0.5368 (4) & $0.2225(2)$ & $0.32414(15)$ & $0.0276(7)$ \\
\hline H11B & 0.637698 & 0.197376 & 0.315255 & $0.033^{*}$ \\
\hline C12B & $0.4184(5)$ & 0.2019 (3) & $0.28402(19)$ & $0.0410(9)$ \\
\hline $\mathrm{H} 12 \mathrm{C}$ & 0.315616 & 0.225699 & 0.291244 & $0.049^{*}$ \\
\hline H12D & 0.436039 & 0.163360 & 0.247952 & $0.049^{*}$ \\
\hline C15B & $0.8579(3)$ & $0.4497(2)$ & $0.35782(13)$ & $0.0167(5)$ \\
\hline C16B & 0.9899 (4) & $0.4966(2)$ & $0.32904(15)$ & $0.0225(6)$ \\
\hline C17B & $0.9330(4)$ & $0.5848(2)$ & $0.30432(15)$ & $0.0246(6)$ \\
\hline H17B & 0.994001 & 0.630332 & 0.282763 & $0.030 *$ \\
\hline C18B & $0.7721(4)$ & $0.5929(2)$ & $0.31718(15)$ & $0.0261(7)$ \\
\hline H18B & 0.706045 & 0.644825 & 0.305485 & $0.031 *$ \\
\hline C19B & $0.7242(4)$ & $0.5117(2)$ & $0.35024(15)$ & $0.0215(6)$ \\
\hline H19B & 0.621223 & 0.499955 & 0.364985 & $0.026^{*}$ \\
\hline $\mathrm{C} 20 \mathrm{~B}$ & $0.7661(4)$ & $0.3503(2)$ & $0.21765(15)$ & $0.0277(7)$ \\
\hline H20B & 0.763789 & 0.289255 & 0.236456 & $0.033^{*}$ \\
\hline C21B & $0.9011(5)$ & 0.3958 (3) & $0.19350(17)$ & $0.0379(9)$ \\
\hline H21B & 1.004752 & 0.370180 & 0.193194 & $0.046^{*}$ \\
\hline $\mathrm{C} 22 \mathrm{~B}$ & $0.8548(6)$ & $0.4862(3)$ & $0.16987(16)$ & $0.0424(10)$ \\
\hline H22B & 0.921530 & 0.531793 & 0.151302 & $0.051 *$ \\
\hline C23B & 0.6919 (6) & 0.4954 (3) & $0.17912(18)$ & 0.0454 (11) \\
\hline H23B & 0.628950 & 0.548636 & 0.167507 & $0.055^{*}$ \\
\hline C24B & $0.6367(5)$ & $0.4115(3)$ & $0.20880(18)$ & $0.0370(9)$ \\
\hline H24B & 0.531268 & 0.399269 & 0.220500 & $0.044^{*}$ \\
\hline $\mathrm{C} 25 \mathrm{~B}$ & $1.1506(4)$ & 0.4595 (3) & $0.32533(19)$ & $0.0378(9)$ \\
\hline $\mathrm{H} 25 \mathrm{~B}$ & 1.167593 & 0.395397 & 0.337110 & $0.045^{*}$ \\
\hline $\mathrm{C} 26 \mathrm{~B}$ & $1.2724(5)$ & $0.5077(4)$ & $0.3071(3)$ & $0.0589(14)$ \\
\hline $\mathrm{H} 26 \mathrm{C}$ & 1.260328 & 0.572105 & 0.294929 & $0.071^{*}$ \\
\hline $\mathrm{H} 26 \mathrm{D}$ & 1.373268 & 0.478476 & 0.305983 & $0.071^{*}$ \\
\hline C34B & 0.9487 (3) & $0.3666(2)$ & $0.47744(13)$ & $0.0173(5)$ \\
\hline C35B & 0.9515 (4) & $0.4587(2)$ & $0.50108(15)$ & $0.0235(6)$ \\
\hline H35B & 0.911046 & 0.509103 & 0.475552 & $0.028^{*}$ \\
\hline $\mathrm{C} 36 \mathrm{~B}$ & $1.0134(4)$ & $0.4770(3)$ & $0.56195(16)$ & $0.0297(7)$ \\
\hline H36B & 1.013790 & 0.539678 & 0.578296 & $0.036^{*}$ \\
\hline C37B & $1.0743(4)$ & $0.4036(3)$ & $0.59841(15)$ & $0.0321(8)$ \\
\hline H37B & 1.116488 & 0.416100 & 0.639959 & $0.039 *$ \\
\hline C38B & 1.0745 (4) & $0.3125(3)$ & $0.57538(15)$ & $0.0300(7)$ \\
\hline H38B & 1.117118 & 0.262545 & 0.600793 & $0.036^{*}$ \\
\hline C39B & $1.0121(4)$ & $0.2941(2)$ & $0.51466(14)$ & $0.0232(6)$ \\
\hline H39B & 1.012919 & 0.231342 & 0.498523 & $0.028^{*}$ \\
\hline
\end{tabular}


Atomic displacement parameters $\left(\AA^{2}\right)$

\begin{tabular}{|c|c|c|c|c|c|c|}
\hline & $U^{11}$ & $U^{22}$ & $U^{33}$ & $U^{12}$ & $U^{13}$ & $U^{23}$ \\
\hline Fe1A & $0.01786(19)$ & $0.0205(2)$ & $0.0162(2)$ & $-0.00154(15)$ & $0.00287(15)$ & $-0.00044(16)$ \\
\hline $\mathrm{Fe} 2 \mathrm{~A}$ & $0.0217(2)$ & $0.0224(2)$ & $0.0172(2)$ & $0.00156(17)$ & $-0.00156(16)$ & $0.00518(17)$ \\
\hline S1A & $0.0204(3)$ & $0.0172(3)$ & $0.0236(4)$ & -0.0040 & $0.0002(3)$ & $-0.0031(3)$ \\
\hline P1A & $0.0148(3)$ & $0.0146(3)$ & $0.0139(3)$ & $-0.0011(2)$ & $0.0003(2)$ & $0.0000(3)$ \\
\hline $\mathrm{C} 1 \mathrm{~A}$ & $0.0163(12)$ & $0.0175(13)$ & $0.0168(13)$ & $-0.0025(10)$ & $0.0010(10)$ & $-0.0007(11)$ \\
\hline $\mathrm{C} 2 \mathrm{~A}$ & 0.0179 (14) & $0.0248(15)$ & $0.0174(14)$ & $-0.0017(11)$ & $0.0001(11)$ & $0.0028(11)$ \\
\hline $\mathrm{C} 3 \mathrm{~A}$ & $0.0174(13)$ & $0.0282(16)$ & $0.0202(15)$ & $-0.0021(12)$ & $0.0015(11)$ & $0.0010(12)$ \\
\hline $\mathrm{C} 4 \mathrm{~A}$ & $0.0226(14)$ & $0.0249(15)$ & $0.0240(16)$ & $-0.0087(12)$ & $0.0036(12)$ & $-0.0057(13)$ \\
\hline C5A & $0.0221(14)$ & $0.0198(13)$ & $0.0219(15)$ & $-0.0039(12)$ & $0.0050(11)$ & $-0.0035(12)$ \\
\hline C6A & $0.0272(16)$ & $0.0371(18)$ & $0.0175(15)$ & $-0.0047(14)$ & $-0.0011(12)$ & $-0.0032(14)$ \\
\hline C7A & 0.0388 (19) & $0.041(2)$ & $0.0251(17)$ & $0.0039(16)$ & $0.0015(14)$ & $-0.0160(16)$ \\
\hline C8A & $0.0300(18)$ & $0.070(3)$ & $0.0203(17)$ & $0.0019(18)$ & $0.0075(13)$ & $-0.0093(18)$ \\
\hline C9A & $0.037(2)$ & $0.057(2)$ & $0.0213(17)$ & $-0.0127(18)$ & $0.0047(15)$ & $0.0087(17)$ \\
\hline $\mathrm{C} 10 \mathrm{~A}$ & $0.0304(18)$ & $0.043(2)$ & $0.0209(16)$ & $-0.0024(15)$ & -0.0030 & $0.0068(15)$ \\
\hline C11A & $0.0235(16)$ & $0.0253(17)$ & $0.047(2)$ & $0.0009(12)$ & 0.0064 (14) & $0.0094(15)$ \\
\hline $\mathrm{C} 12 \mathrm{~A}$ & $0.0326(19)$ & $0.0250(18)$ & 0.075 & $0.0052(15)$ & $0.0124(19)$ & 0.0104 (19) \\
\hline C15A & $0.0183(12)$ & $0.0147(13)$ & $0.0165(13)$ & $0.0004(10)$ & $-0.0005(10)$ & $0.0012(11)$ \\
\hline C16A & $0.0218(14)$ & $0.0227(14)$ & $0.0153(13)$ & $0.0029(11)$ & $-0.0007(10)$ & $0.0014(11)$ \\
\hline C17A & $0.0279(16)$ & $0.0308(17)$ & $0.0168(14)$ & $0.0054(13)$ & $0.0038(12)$ & $-0.0003(13)$ \\
\hline C18A & $0.0315(17)$ & $0.0266(15)$ & $0.0174(15)$ & 0.0013 (13) & $-0.0044(13)$ & $-0.0024(12)$ \\
\hline C19A & $0.0205(14)$ & $0.0211(14)$ & $0.0183(14)$ & $-0.0009(11)$ & $-0.0025(11)$ & $0.0005(11)$ \\
\hline $\mathrm{C} 20 \mathrm{~A}$ & $0.050(2)$ & $0.0211(16)$ & $0.038(2)$ & $0.0080(15)$ & $0.0013(17)$ & $0.0050(15)$ \\
\hline $\mathrm{C} 21 \mathrm{~A}$ & $0.038(2)$ & $0.0249(17)$ & $0.048(2)$ & $-0.0015(15)$ & $0.0046(17)$ & $0.0132(16)$ \\
\hline $\mathrm{C} 22 \mathrm{~A}$ & $0.052(2)$ & 0.0340 (19) & $0.032(2)$ & $0.0101(17)$ & 0.0047 (17) & $0.0196(16)$ \\
\hline $\mathrm{C} 23 \mathrm{~A}$ & $0.042(2)$ & $0.037(2)$ & $0.037(2)$ & $0.0070(16)$ & $-0.0144(17)$ & $0.0147(16)$ \\
\hline $\mathrm{C} 24 \mathrm{~A}$ & $0.0331(18)$ & $0.0302(18)$ & $0.052(2)$ & $0.0112(15)$ & $0.0042(17)$ & $0.0125(17)$ \\
\hline $\mathrm{C} 25 \mathrm{~A}$ & $0.0216(14)$ & $0.0384(18)$ & $0.0204(15)$ & $0.0005(13)$ & $-0.0007(12)$ & 0.0009 (14) \\
\hline $\mathrm{C} 26 \mathrm{~A}$ & $0.0217(16)$ & $0.073(3)$ & $0.033(2)$ & $-0.0001(18)$ & 0.0025 (14) & $-0.007(2)$ \\
\hline C34A & $0.0143(12)$ & $0.0185(13)$ & $0.0173(13)$ & $-0.0009(10)$ & $0.0016(10)$ & $0.0018(11)$ \\
\hline $\mathrm{C} 35 \mathrm{~A}$ & $0.0221(14)$ & $0.0244(15)$ & $0.0159(14)$ & $-0.0006(12)$ & $0.0014(11)$ & $0.0022(12)$ \\
\hline C36A & $0.0238(15)$ & $0.0310(16)$ & $0.0193(15)$ & $0.0006(13)$ & $-0.0013(12)$ & $0.0072(13)$ \\
\hline C37A & $0.0229(15)$ & $0.0218(15)$ & $0.0318(18)$ & $0.0021(12)$ & $0.0023(13)$ & $0.0099(13)$ \\
\hline C38A & $0.0273(16)$ & $0.0191(14)$ & $0.0309(18)$ & $0.0016(12)$ & $0.0003(13)$ & $0.0012(13)$ \\
\hline C39A & $0.0233(15)$ & $0.0199(14)$ & $0.0229(15)$ & $-0.0001(11)$ & $-0.0022(12)$ & $0.0001(12)$ \\
\hline Fe1B & $0.0214(2)$ & 0.01669 (19) & $0.0186(2)$ & $-0.00228(16)$ & $0.00382(16)$ & $0.00082(17)$ \\
\hline $\mathrm{Fe} 2 \mathrm{~B}$ & $0.0239(2)$ & $0.01526(19)$ & $0.01395(19)$ & $0.00233(16)$ & $-0.00102(15)$ & $0.00088(16)$ \\
\hline S1B & $0.0253(3)$ & $0.0184(3)$ & $0.0198(4)$ & $0.0053(3)$ & 0.0009 & $-0.0027(3)$ \\
\hline P1B & $0.0159(3)$ & $0.0139(3)$ & $0.0129(3)$ & $0.0000(2)$ & $-0.0004(3)$ & $-0.0002(3)$ \\
\hline C1B & $0.0156(13)$ & $0.0144(12)$ & $0.0192(14)$ & $0.0004(10)$ & $0.0002(10)$ & $0.0024(11)$ \\
\hline $\mathrm{C} 2 \mathrm{~B}$ & $0.0211(14)$ & $0.0219(14)$ & $0.0217(15)$ & $-0.0046(12)$ & $-0.0023(11)$ & $0.0052(12)$ \\
\hline $\mathrm{C} 3 \mathrm{~B}$ & $0.0180(14)$ & $0.0252(16)$ & $0.0364(19)$ & $-0.0037(12)$ & $-0.0020(13)$ & 0.0067 (14) \\
\hline C4B & 0.0197 (14) & $0.0209(15)$ & $0.043(2)$ & $0.0014(12)$ & $0.0064(13)$ & $0.0007(14)$ \\
\hline C5B & $0.0220(14)$ & $0.0176(14)$ & 0.0307 (17) & $-0.0002(11)$ & $0.0045(12)$ & $-0.0038(12)$ \\
\hline C6B & $0.046(2)$ & $0.0219(15)$ & 0.0324 (19) & $0.0095(15)$ & $0.0063(16)$ & $0.0082(14)$ \\
\hline $\mathrm{C} 7 \mathrm{~B}$ & $0.081(3)$ & $0.0175(16)$ & $0.032(2)$ & $-0.0041(18)$ & $-0.002(2)$ & $0.0038(15)$ \\
\hline
\end{tabular}




$\begin{array}{lllllll}\text { C8B } & 0.045(2) & 0.038(2) & 0.071(3) & -0.0213(18) & -0.002(2) & 0.029(2) \\ \text { C9B } & 0.056(3) & 0.043(2) & 0.036(2) & -0.0007(19) & 0.0186(19) & 0.0113(19) \\ \text { C10B } & 0.056(2) & 0.037(2) & 0.0202(17) & 0.0078(17) & 0.0025(16) & 0.0074(15) \\ \text { C11B } & 0.0306(16) & 0.0288(16) & 0.0232(16) & -0.0076(13) & -0.0019(13) & 0.0043(13) \\ \text { C12B } & 0.044(2) & 0.045(2) & 0.033(2) & -0.0086(17) & -0.0111(17) & -0.0043(17) \\ \text { C15B } & 0.0200(13) & 0.0163(13) & 0.0139(13) & -0.0007(10) & 0.0003(10) & 0.0004(10) \\ \text { C16B } & 0.0225(14) & 0.0239(15) & 0.0211(15) & -0.0057(12) & -0.0015(12) & 0.0048(12) \\ \text { C17B } & 0.0346(17) & 0.0190(14) & 0.0201(15) & -0.0079(13) & -0.0015(13) & 0.0026(12) \\ \text { C18B } & 0.0415(19) & 0.0151(14) & 0.0220(16) & 0.0049(12) & 0.0053(14) & -0.0007(12) \\ \text { C19B } & 0.0260(15) & 0.0171(13) & 0.0215(15) & 0.0043(11) & 0.0052(12) & 0.0003(12) \\ \text { C20B } & 0.048(2) & 0.0199(15) & 0.0150(14) & 0.0001(14) & -0.0025(13) & -0.0033(12) \\ \text { C21B } & 0.062(2) & 0.0283(18) & 0.0248(17) & 0.0006(17) & 0.0159(17) & -0.0055(15) \\ \text { C22B } & 0.083(3) & 0.0294(19) & 0.0151(16) & -0.0097(19) & 0.0058(17) & 0.0034(15) \\ \text { C23B } & 0.080(3) & 0.0237(18) & 0.030(2) & -0.0011(18) & -0.028(2) & 0.0049(15) \\ \text { C24B } & 0.044(2) & 0.0324(18) & 0.0337(19) & -0.0021(15) & -0.0188(17) & -0.0024(15) \\ \text { C25B } & 0.0209(16) & 0.049(2) & 0.044(2) & 0.0001(15) & -0.0007(15) & 0.0204(18) \\ \text { C26B } & 0.026(2) & 0.069(3) & 0.082(4) & -0.007(2) & 0.007(2) & 0.024(3) \\ \text { C34B } & 0.0149(12) & 0.0227(14) & 0.0143(13) & -0.0003(10) & 0.0013(10) & 0.0002(11) \\ \text { C35B } & 0.0265(15) & 0.0237(15) & 0.0204(15) & -0.0032(12) & 0.0009(12) & -0.0008(12) \\ \text { C36B } & 0.0325(17) & 0.0344(17) & 0.0225(16) & -0.0091(15) & 0.0035(13) & -0.0099(15) \\ \text { C37B } & 0.0254(16) & 0.056(2) & 0.0148(14) & -0.0070(15) & 0.0002(12) & -0.0051(15) \\ \text { C38B } & 0.0273(16) & 0.044(2) & 0.0190(16) & 0.0052(14) & -0.0014(13) & 0.0073(15) \\ \text { C39B } & 0.0212(14) & 0.0293(16) & 0.0189(14) & 0.0037(12) & -0.0014(11) & -0.0005(13) \\ & & & & & & \end{array}$

Geometric parameters $\left(\AA,{ }^{\circ}\right)$

\begin{tabular}{|c|c|c|c|}
\hline $\mathrm{Fe} 1 \mathrm{~A}-\mathrm{C} 1 \mathrm{~A}$ & $2.012(3)$ & $\mathrm{Fe} 1 \mathrm{~B}-\mathrm{C} 1 \mathrm{~B}$ & $2.015(3)$ \\
\hline $\mathrm{Fe} 1 \mathrm{~A}-\mathrm{C} 2 \mathrm{~A}$ & $2.048(3)$ & $\mathrm{Fe} 1 \mathrm{~B}-\mathrm{C} 2 \mathrm{~B}$ & $2.033(3)$ \\
\hline $\mathrm{Fe} 1 \mathrm{~A}-\mathrm{C} 3 \mathrm{~A}$ & $2.045(3)$ & $\mathrm{Fe} 1 \mathrm{~B}-\mathrm{C} 3 \mathrm{~B}$ & $2.034(3)$ \\
\hline $\mathrm{Fe} 1 \mathrm{~A}-\mathrm{C} 4 \mathrm{~A}$ & $2.060(3)$ & $\mathrm{Fe} 1 \mathrm{~B}-\mathrm{C} 4 \mathrm{~B}$ & $2.056(3)$ \\
\hline $\mathrm{Fe} 1 \mathrm{~A}-\mathrm{C} 5 \mathrm{~A}$ & $2.038(3)$ & $\mathrm{Fe} 1 \mathrm{~B}-\mathrm{C} 5 \mathrm{~B}$ & $2.031(3)$ \\
\hline $\mathrm{Fe} 1 \mathrm{~A}-\mathrm{C} 6 \mathrm{~A}$ & $2.035(3)$ & $\mathrm{Fe} 1 \mathrm{~B}-\mathrm{C} 6 \mathrm{~B}$ & $2.037(3)$ \\
\hline $\mathrm{Fe} 1 \mathrm{~A}-\mathrm{C} 7 \mathrm{~A}$ & $2.032(3)$ & $\mathrm{Fe} 1 \mathrm{~B}-\mathrm{C} 7 \mathrm{~B}$ & $2.035(4)$ \\
\hline $\mathrm{Fe} 1 \mathrm{~A}-\mathrm{C} 8 \mathrm{~A}$ & $2.035(4)$ & $\mathrm{Fe} 1 \mathrm{~B}-\mathrm{C} 8 \mathrm{~B}$ & $2.021(4)$ \\
\hline $\mathrm{Fe} 1 \mathrm{~A}-\mathrm{C} 9 \mathrm{~A}$ & 2.049 (4) & $\mathrm{Fe} 1 \mathrm{~B}-\mathrm{C} 9 \mathrm{~B}$ & $2.031(4)$ \\
\hline $\mathrm{Fe} 1 \mathrm{~A}-\mathrm{C} 10 \mathrm{~A}$ & $2.052(3)$ & $\mathrm{Fe} 1 \mathrm{~B}-\mathrm{C} 10 \mathrm{~B}$ & 2.049 (4) \\
\hline $\mathrm{Fe} 2 \mathrm{~A}-\mathrm{C} 15 \mathrm{~A}$ & $2.027(3)$ & $\mathrm{Fe} 2 \mathrm{~B}-\mathrm{C} 15 \mathrm{~B}$ & $2.032(3)$ \\
\hline $\mathrm{Fe} 2 \mathrm{~A}-\mathrm{C} 16 \mathrm{~A}$ & $2.043(3)$ & $\mathrm{Fe} 2 \mathrm{~B}-\mathrm{C} 16 \mathrm{~B}$ & $2.039(3)$ \\
\hline $\mathrm{Fe} 2 \mathrm{~A}-\mathrm{C} 17 \mathrm{~A}$ & $2.038(3)$ & $\mathrm{Fe} 2 \mathrm{~B}-\mathrm{C} 17 \mathrm{~B}$ & $2.038(3)$ \\
\hline $\mathrm{Fe} 2 \mathrm{~A}-\mathrm{C} 18 \mathrm{~A}$ & $2.053(3)$ & $\mathrm{Fe} 2 \mathrm{~B}-\mathrm{C} 18 \mathrm{~B}$ & $2.034(3)$ \\
\hline $\mathrm{Fe} 2 \mathrm{~A}-\mathrm{C} 19 \mathrm{~A}$ & $2.046(3)$ & $\mathrm{Fe} 2 \mathrm{~B}-\mathrm{C} 19 \mathrm{~B}$ & $2.037(3)$ \\
\hline $\mathrm{Fe} 2 \mathrm{~A}-\mathrm{C} 20 \mathrm{~A}$ & $2.055(4)$ & $\mathrm{Fe} 2 \mathrm{~B}-\mathrm{C} 20 \mathrm{~B}$ & $2.046(3)$ \\
\hline $\mathrm{Fe} 2 \mathrm{~A}-\mathrm{C} 21 \mathrm{~A}$ & $2.050(4)$ & $\mathrm{Fe} 2 \mathrm{~B}-\mathrm{C} 21 \mathrm{~B}$ & $2.037(3)$ \\
\hline $\mathrm{Fe} 2 \mathrm{~A}-\mathrm{C} 22 \mathrm{~A}$ & $2.035(3)$ & $\mathrm{Fe} 2 \mathrm{~B}-\mathrm{C} 22 \mathrm{~B}$ & $2.038(4)$ \\
\hline $\mathrm{Fe} 2 \mathrm{~A}-\mathrm{C} 23 \mathrm{~A}$ & $2.040(3)$ & $\mathrm{Fe} 2 \mathrm{~B}-\mathrm{C} 23 \mathrm{~B}$ & $2.037(3)$ \\
\hline $\mathrm{Fe} 2 \mathrm{~A}-\mathrm{C} 24 \mathrm{~A}$ & $2.044(4)$ & $\mathrm{Fe} 2 \mathrm{~B}-\mathrm{C} 24 \mathrm{~B}$ & $2.048(3)$ \\
\hline $\mathrm{S} 1 \mathrm{~A}-\mathrm{P} 1 \mathrm{~A}$ & $1.9600(10)$ & $\mathrm{S} 1 \mathrm{~B}-\mathrm{P} 1 \mathrm{~B}$ & $1.9563(10)$ \\
\hline $\mathrm{P} 1 \mathrm{~A}-\mathrm{C} 1 \mathrm{~A}$ & $1.794(3)$ & $\mathrm{P} 1 \mathrm{~B}-\mathrm{C} 1 \mathrm{~B}$ & $1.792(3)$ \\
\hline
\end{tabular}




\begin{tabular}{|c|c|c|c|}
\hline $\mathrm{P} 1 \mathrm{~A}-\mathrm{C} 15 \mathrm{~A}$ & $1.787(3)$ & $\mathrm{P} 1 \mathrm{~B}-\mathrm{C} 15 \mathrm{~B}$ & $1.796(3)$ \\
\hline $\mathrm{P} 1 \mathrm{~A}-\mathrm{C} 34 \mathrm{~A}$ & $1.821(3)$ & $\mathrm{P} 1 \mathrm{~B}-\mathrm{C} 34 \mathrm{~B}$ & $1.819(3)$ \\
\hline $\mathrm{C} 1 \mathrm{~A}-\mathrm{C} 2 \mathrm{~A}$ & $1.451(4)$ & $\mathrm{C} 1 \mathrm{~B}-\mathrm{C} 2 \mathrm{~B}$ & $1.449(4)$ \\
\hline $\mathrm{C} 1 \mathrm{~A}-\mathrm{C} 5 \mathrm{~A}$ & $1.432(4)$ & $\mathrm{C} 1 \mathrm{~B}-\mathrm{C} 5 \mathrm{~B}$ & $1.435(4)$ \\
\hline $\mathrm{C} 2 \mathrm{~A}-\mathrm{C} 3 \mathrm{~A}$ & $1.431(4)$ & $\mathrm{C} 2 \mathrm{~B}-\mathrm{C} 3 \mathrm{~B}$ & $1.430(4)$ \\
\hline $\mathrm{C} 2 \mathrm{~A}-\mathrm{C} 11 \mathrm{~A}$ & $1.457(4)$ & $\mathrm{C} 2 \mathrm{~B}-\mathrm{C} 11 \mathrm{~B}$ & $1.466(5)$ \\
\hline $\mathrm{C} 3 \mathrm{~A}-\mathrm{H} 3 \mathrm{~A}$ & 0.9500 & $\mathrm{C} 3 \mathrm{~B}-\mathrm{H} 3 \mathrm{~B}$ & 0.9500 \\
\hline $\mathrm{C} 3 \mathrm{~A}-\mathrm{C} 4 \mathrm{~A}$ & $1.416(5)$ & $\mathrm{C} 3 \mathrm{~B}-\mathrm{C} 4 \mathrm{~B}$ & $1.404(5)$ \\
\hline $\mathrm{C} 4 \mathrm{~A}-\mathrm{H} 4 \mathrm{~A}$ & 0.9500 & $\mathrm{C} 4 \mathrm{~B}-\mathrm{H} 4 \mathrm{~B}$ & 0.9500 \\
\hline $\mathrm{C} 4 \mathrm{~A}-\mathrm{C} 5 \mathrm{~A}$ & $1.417(4)$ & $\mathrm{C} 4 \mathrm{~B}-\mathrm{C} 5 \mathrm{~B}$ & $1.414(4)$ \\
\hline $\mathrm{C} 5 \mathrm{~A}-\mathrm{H} 5 \mathrm{~A}$ & 0.9500 & $\mathrm{C} 5 \mathrm{~B}-\mathrm{H} 5 \mathrm{~B}$ & 0.9500 \\
\hline C6A-H6A & 0.9500 & $\mathrm{C} 6 \mathrm{~B}-\mathrm{H} 6 \mathrm{~B}$ & 0.9500 \\
\hline $\mathrm{C} 6 \mathrm{~A}-\mathrm{C} 7 \mathrm{~A}$ & $1.407(5)$ & $\mathrm{C} 6 \mathrm{~B}-\mathrm{C} 7 \mathrm{~B}$ & $1.385(6)$ \\
\hline $\mathrm{C} 6 \mathrm{~A}-\mathrm{C} 10 \mathrm{~A}$ & $1.416(5)$ & $\mathrm{C} 6 \mathrm{~B}-\mathrm{C} 10 \mathrm{~B}$ & $1.400(5)$ \\
\hline $\mathrm{C} 7 \mathrm{~A}-\mathrm{H} 7 \mathrm{~A}$ & 0.9500 & $\mathrm{C} 7 \mathrm{~B}-\mathrm{H} 7 \mathrm{~B}$ & 0.9500 \\
\hline $\mathrm{C} 7 \mathrm{~A}-\mathrm{C} 8 \mathrm{~A}$ & $1.413(6)$ & $\mathrm{C} 7 \mathrm{~B}-\mathrm{C} 8 \mathrm{~B}$ & $1.443(6)$ \\
\hline $\mathrm{C} 8 \mathrm{~A}-\mathrm{H} 8 \mathrm{~A}$ & 0.9500 & $\mathrm{C} 8 \mathrm{~B}-\mathrm{H} 8 \mathrm{~B}$ & 0.9500 \\
\hline $\mathrm{C} 8 \mathrm{~A}-\mathrm{C} 9 \mathrm{~A}$ & $1.411(6)$ & $\mathrm{C} 8 \mathrm{~B}-\mathrm{C} 9 \mathrm{~B}$ & $1.409(6)$ \\
\hline C9A-H9A & 0.9500 & C9B-H9B & 0.9500 \\
\hline $\mathrm{C} 9 \mathrm{~A}-\mathrm{C} 10 \mathrm{~A}$ & $1.420(5)$ & $\mathrm{C} 9 \mathrm{~B}-\mathrm{C} 10 \mathrm{~B}$ & $1.385(6)$ \\
\hline $\mathrm{C} 10 \mathrm{~A}-\mathrm{H} 10 \mathrm{~A}$ & 0.9500 & $\mathrm{C} 10 \mathrm{~B}-\mathrm{H} 10 \mathrm{~B}$ & 0.9500 \\
\hline $\mathrm{C} 11 \mathrm{~A}-\mathrm{H} 11 \mathrm{~A}$ & 0.9500 & $\mathrm{C} 11 \mathrm{~B}-\mathrm{H} 11 \mathrm{~B}$ & 0.9500 \\
\hline $\mathrm{C} 11 \mathrm{~A}-\mathrm{C} 12 \mathrm{~A}$ & $1.321(5)$ & $\mathrm{C} 11 \mathrm{~B}-\mathrm{C} 12 \mathrm{~B}$ & $1.322(5)$ \\
\hline $\mathrm{C} 12 \mathrm{~A}-\mathrm{H} 12 \mathrm{~A}$ & 0.9500 & $\mathrm{C} 12 \mathrm{~B}-\mathrm{H} 12 \mathrm{C}$ & 0.9500 \\
\hline $\mathrm{C} 12 \mathrm{~A}-\mathrm{H} 12 \mathrm{~B}$ & 0.9500 & $\mathrm{C} 12 \mathrm{~B}-\mathrm{H} 12 \mathrm{D}$ & 0.9500 \\
\hline $\mathrm{C} 15 \mathrm{~A}-\mathrm{C} 16 \mathrm{~A}$ & $1.445(4)$ & $\mathrm{C} 15 \mathrm{~B}-\mathrm{C} 16 \mathrm{~B}$ & $1.450(4)$ \\
\hline $\mathrm{C} 15 \mathrm{~A}-\mathrm{C} 19 \mathrm{~A}$ & $1.438(4)$ & $\mathrm{C} 15 \mathrm{~B}-\mathrm{C} 19 \mathrm{~B}$ & $1.437(4)$ \\
\hline $\mathrm{C} 16 \mathrm{~A}-\mathrm{C} 17 \mathrm{~A}$ & $1.422(4)$ & $\mathrm{C} 16 \mathrm{~B}-\mathrm{C} 17 \mathrm{~B}$ & $1.429(4)$ \\
\hline $\mathrm{C} 16 \mathrm{~A}-\mathrm{C} 25 \mathrm{~A}$ & $1.462(4)$ & $\mathrm{C} 16 \mathrm{~B}-\mathrm{C} 25 \mathrm{~B}$ & $1.463(5)$ \\
\hline $\mathrm{C} 17 \mathrm{~A}-\mathrm{H} 17 \mathrm{~A}$ & 0.9500 & $\mathrm{C} 17 \mathrm{~B}-\mathrm{H} 17 \mathrm{~B}$ & 0.9500 \\
\hline $\mathrm{C} 17 \mathrm{~A}-\mathrm{C} 18 \mathrm{~A}$ & $1.416(5)$ & $\mathrm{C} 17 \mathrm{~B}-\mathrm{C} 18 \mathrm{~B}$ & $1.404(5)$ \\
\hline $\mathrm{C} 18 \mathrm{~A}-\mathrm{H} 18 \mathrm{~A}$ & 0.9500 & $\mathrm{C} 18 \mathrm{~B}-\mathrm{H} 18 \mathrm{~B}$ & 0.9500 \\
\hline $\mathrm{C} 18 \mathrm{~A}-\mathrm{C} 19 \mathrm{~A}$ & $1.415(4)$ & $\mathrm{C} 18 \mathrm{~B}-\mathrm{C} 19 \mathrm{~B}$ & 1.409 (4) \\
\hline $\mathrm{C} 19 \mathrm{~A}-\mathrm{H} 19 \mathrm{~A}$ & 0.9500 & $\mathrm{C} 19 \mathrm{~B}-\mathrm{H} 19 \mathrm{~B}$ & 0.9500 \\
\hline $\mathrm{C} 20 \mathrm{~A}-\mathrm{H} 20 \mathrm{~A}$ & 0.9500 & $\mathrm{C} 20 \mathrm{~B}-\mathrm{H} 20 \mathrm{~B}$ & 0.9500 \\
\hline $\mathrm{C} 20 \mathrm{~A}-\mathrm{C} 21 \mathrm{~A}$ & $1.418(6)$ & $\mathrm{C} 20 \mathrm{~B}-\mathrm{C} 21 \mathrm{~B}$ & $1.420(5)$ \\
\hline $\mathrm{C} 20 \mathrm{~A}-\mathrm{C} 24 \mathrm{~A}$ & $1.406(6)$ & $\mathrm{C} 20 \mathrm{~B}-\mathrm{C} 24 \mathrm{~B}$ & $1.405(5)$ \\
\hline $\mathrm{C} 21 \mathrm{~A}-\mathrm{H} 21 \mathrm{~A}$ & 0.9500 & $\mathrm{C} 21 \mathrm{~B}-\mathrm{H} 21 \mathrm{~B}$ & 0.9500 \\
\hline $\mathrm{C} 21 \mathrm{~A}-\mathrm{C} 22 \mathrm{~A}$ & $1.397(6)$ & $\mathrm{C} 21 \mathrm{~B}-\mathrm{C} 22 \mathrm{~B}$ & $1.421(5)$ \\
\hline $\mathrm{C} 22 \mathrm{~A}-\mathrm{H} 22 \mathrm{~A}$ & 0.9500 & $\mathrm{C} 22 \mathrm{~B}-\mathrm{H} 22 \mathrm{~B}$ & 0.9500 \\
\hline $\mathrm{C} 22 \mathrm{~A}-\mathrm{C} 23 \mathrm{~A}$ & $1.416(6)$ & $\mathrm{C} 22 \mathrm{~B}-\mathrm{C} 23 \mathrm{~B}$ & $1.407(6)$ \\
\hline $\mathrm{C} 23 \mathrm{~A}-\mathrm{H} 23 \mathrm{~A}$ & 0.9500 & $\mathrm{C} 23 \mathrm{~B}-\mathrm{H} 23 \mathrm{~B}$ & 0.9500 \\
\hline $\mathrm{C} 23 \mathrm{~A}-\mathrm{C} 24 \mathrm{~A}$ & $1.419(6)$ & $\mathrm{C} 23 \mathrm{~B}-\mathrm{C} 24 \mathrm{~B}$ & $1.426(5)$ \\
\hline $\mathrm{C} 24 \mathrm{~A}-\mathrm{H} 24 \mathrm{~A}$ & 0.9500 & $\mathrm{C} 24 \mathrm{~B}-\mathrm{H} 24 \mathrm{~B}$ & 0.9500 \\
\hline $\mathrm{C} 25 \mathrm{~A}-\mathrm{H} 25 \mathrm{~A}$ & 0.9500 & $\mathrm{C} 25 \mathrm{~B}-\mathrm{H} 25 \mathrm{~B}$ & 0.9500 \\
\hline $\mathrm{C} 25 \mathrm{~A}-\mathrm{C} 26 \mathrm{~A}$ & $1.314(5)$ & $\mathrm{C} 25 \mathrm{~B}-\mathrm{C} 26 \mathrm{~B}$ & $1.305(5)$ \\
\hline $\mathrm{C} 26 \mathrm{~A}-\mathrm{H} 26 \mathrm{~A}$ & 0.9500 & $\mathrm{C} 26 \mathrm{~B}-\mathrm{H} 26 \mathrm{C}$ & 0.9500 \\
\hline $\mathrm{C} 26 \mathrm{~A}-\mathrm{H} 26 \mathrm{~B}$ & 0.9500 & $\mathrm{C} 26 \mathrm{~B}-\mathrm{H} 26 \mathrm{D}$ & 0.9500 \\
\hline
\end{tabular}




C34A-C35A
C34A-C39A
C35A-H35A
C35A-C36A
C36A-H36A
C36A-C37A
C37A-H37A
C37A-C38A
C38A-H38A
C38A-C39A
C39A-H39A
C1A-Fe1A-C2A
C1A-Fe1A-C3A
C1A-Fe1A-C4A
C1A-Fe1A-C5A
C1A-Fe1A-C6A
C1A-Fe1A-C7A
C1A-Fe1A-C8A
C1A-Fe1A-C9A
C1A-Fe1A-C10A
C2A-Fe1A-C4A
C2A-Fe1A-C9A
C2A-Fe1A-C10A
C3A-Fe1A-C2A
C3A-Fe1A-C4A
C3A-Fe1A-C9A
C3A-Fe1A-C10A
C5A-Fe1A-C2A
C5A-Fe1A-C3A
C5A-Fe1A-C4A
C5A-Fe1A-C9A
C5A-Fe1A-C10A
C6A-Fe1A-C2A
C6A-Fe1A-C3A
C6A-Fe1A-C4A
C6A-Fe1A-C5A
C6A-Fe1A-C8A
C6A-Fe1A-C9A
C6A-Fe1A-C10A
C7A-Fe1A-C2A
C7A-Fe1A-C3A
C7A-Fe1A-C4A
C7A-Fe1A-C5A
C7A-Fe1A-C6A
C7A-Fe1A-C8A
C7A-Fe1A-C9A
C7A-Fe1A-C10A

$1.393(4)$
$1.396(4)$
0.9500
$1.387(4)$
0.9500
$1.386(5)$
0.9500
$1.373(5)$
0.9500
$1.395(4)$
0.9500

$41.88(11)$

$69.45(11)$

$69.03(12)$

$41.41(11)$

$108.47(13)$

$125.59(14)$

$162.38(16)$

$156.06(15)$

$121.38(13)$

$68.68(12)$

$160.94(15)$

$156.06(13)$

40.91 (11)

40.35 (13)

$124.40(14)$

$162.62(13)$

$69.51(12)$

$68.50(12)$

$40.46(12)$

$120.75(16)$

$109.61(14)$

$120.05(13)$

$154.00(14)$

$164.96(14)$

$128.04(13)$

$68.11(14)$

$68.13(15)$

$40.52(14)$

$105.96(14)$

$118.34(14)$

$153.03(14)$

$164.61(14)$

40.50 (14)

$40.65(16)$

$68.24(17)$

$68.22(16)$

$\begin{array}{ll}\text { C34B-C35B } & 1.393(4) \\ \text { C34B-C39B } & 1.388(4) \\ \text { C35B-H35B } & 0.9500 \\ \text { C35B-C36B } & 1.392(4) \\ \text { C36B-H36B } & 0.9500 \\ \text { C36B-C37B } & 1.381(5) \\ \text { C37B-H37B } & 0.9500 \\ \text { C37B-C38B } & 1.376(5) \\ \text { C38B-H38B } & 0.9500 \\ \text { C38B-C39B } & 1.391(4) \\ \text { C39B-H39B } & 0.9500\end{array}$

C1B-Fe1B-C2B $\quad 41.95$ (11)

$\mathrm{C} 1 \mathrm{~B}-\mathrm{Fe} 1 \mathrm{~B}-\mathrm{C} 3 \mathrm{~B} \quad 69.53(12)$

$\mathrm{C} 1 \mathrm{~B}-\mathrm{Fe} 1 \mathrm{~B}-\mathrm{C} 4 \mathrm{~B} \quad 69.11$ (12)

$\mathrm{C} 1 \mathrm{~B}-\mathrm{Fe} 1 \mathrm{~B}-\mathrm{C} 5 \mathrm{~B} \quad 41.55$ (12)

C1B-Fe1B-C6B 111.05 (14)

C1B-Fe1B-C7B 126.99 (16)

C1B-Fe1B-C8B 163.84 (17)

$\mathrm{C} 1 \mathrm{~B}-\mathrm{Fe} 1 \mathrm{~B}-\mathrm{C} 9 \mathrm{~B} \quad 155.05(15)$

$\mathrm{C} 1 \mathrm{~B}-\mathrm{Fe} 1 \mathrm{~B}-\mathrm{C} 10 \mathrm{~B} \quad 122.64(15)$

$\mathrm{C} 2 \mathrm{~B}-\mathrm{Fe} 1 \mathrm{~B}-\mathrm{C} 3 \mathrm{~B} \quad 41.16(13)$

$\mathrm{C} 2 \mathrm{~B}-\mathrm{Fe} 1 \mathrm{~B}-\mathrm{C} 4 \mathrm{~B} \quad 68.82(14)$

C2B-Fe1B-C6B 123.05 (14)

$\mathrm{C} 2 \mathrm{~B}-\mathrm{Fe} 1 \mathrm{~B}-\mathrm{C} 7 \mathrm{~B} \quad 107.65(14)$

C2B-Fe1B-C10B $158.35(15)$

$\mathrm{C} 3 \mathrm{~B}-\mathrm{Fe} 1 \mathrm{~B}-\mathrm{C} 4 \mathrm{~B} \quad 40.15$ (14)

C3B-Fe1B-C6B $156.26(14)$

$\mathrm{C} 3 \mathrm{~B}-\mathrm{Fe} 1 \mathrm{~B}-\mathrm{C} 7 \mathrm{~B} \quad 119.73(15)$

C3B-Fe1B-C10B $159.93(15)$

$\mathrm{C} 5 \mathrm{~B}-\mathrm{Fe} 1 \mathrm{~B}-\mathrm{C} 2 \mathrm{~B} \quad 69.75(13)$

$\mathrm{C} 5 \mathrm{~B}-\mathrm{Fe} 1 \mathrm{~B}-\mathrm{C} 3 \mathrm{~B} \quad 68.46(13)$

$\mathrm{C} 5 \mathrm{~B}-\mathrm{Fe} 1 \mathrm{~B}-\mathrm{C} 4 \mathrm{~B} \quad 40.49$ (12)

C5B-Fe1B-C6B $128.71(14)$

C5B-Fe1B-C7B $165.29(16)$

C5B-Fe1B-C9B $118.51(16)$

C5B-Fe1B-C10B $108.93(15)$

C6B-Fe1B-C4B $163.54(15)$

$\mathrm{C} 6 \mathrm{~B}-\mathrm{Fe} 1 \mathrm{~B}-\mathrm{C} 10 \mathrm{~B} \quad 40.06(15)$

C7B-Fe1B-C4B $153.31(16)$

$\mathrm{C} 7 \mathrm{~B}-\mathrm{Fe} 1 \mathrm{~B}-\mathrm{C} 6 \mathrm{~B} \quad 39.77(16)$

$\mathrm{C} 7 \mathrm{~B}-\mathrm{Fe} 1 \mathrm{~B}-\mathrm{C} 10 \mathrm{~B} \quad 67.83(16)$

$\mathrm{C} 8 \mathrm{~B}-\mathrm{Fe} 1 \mathrm{~B}-\mathrm{C} 2 \mathrm{~B} \quad 124.04$ (16)

$\mathrm{C} 8 \mathrm{~B}-\mathrm{Fe} 1 \mathrm{~B}-\mathrm{C} 3 \mathrm{~B} \quad 104.77$ (16)

$\mathrm{C} 8 \mathrm{~B}-\mathrm{Fe} 1 \mathrm{~B}-\mathrm{C} 4 \mathrm{~B} \quad 116.88(16)$

C8B-Fe1B-C5B $151.88(18)$

$\mathrm{C} 8 \mathrm{~B}-\mathrm{Fe} 1 \mathrm{~B}-\mathrm{C} 6 \mathrm{~B} \quad 67.79(17)$

$\mathrm{C} 8 \mathrm{~B}-\mathrm{Fe} 1 \mathrm{~B}-\mathrm{C} 7 \mathrm{~B} \quad 41.68(19)$ 


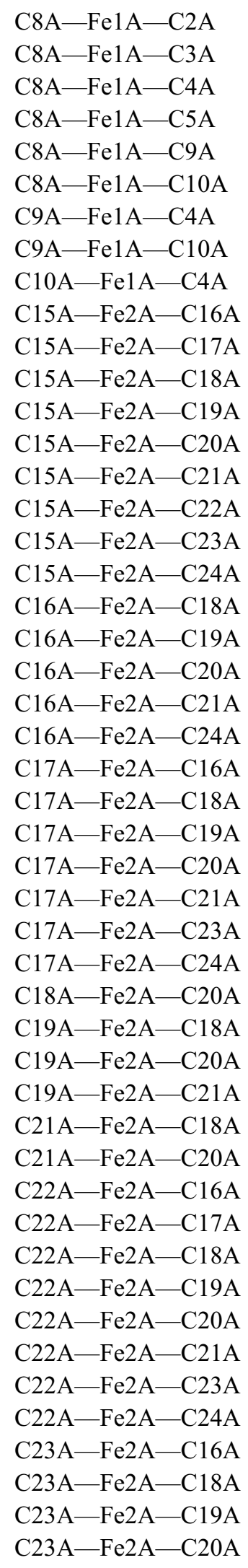

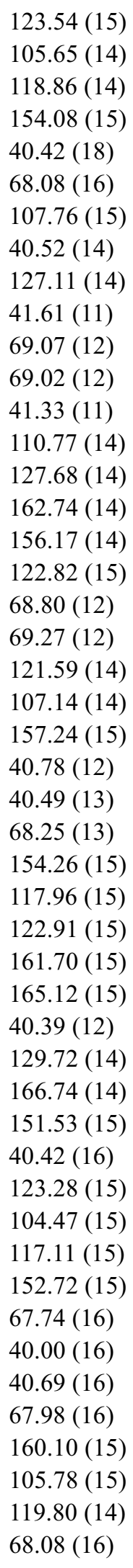

$123.54(15)$

$105.65(14)$

$118.86(14)$

$154.08(15)$

$40.42(18)$

$68.08(16)$

$107.76(15)$

$40.52(14)$

$127.11(14)$

41.61 (11)

$69.07(12)$

$69.02(12)$

$41.33(11)$

$110.77(14)$

$127.68(14)$

$162.74(14)$

$156.17(14)$

$122.82(15)$

$68.80(12)$

$69.27(12)$

$121.59(14)$

$107.14(14)$

$157.24(15)$

40.78 (12)

40.49 (13)

68.25 (13)

$154.26(15)$

$117.96(15)$

$122.91(15)$

$161.70(15)$

$165.12(15)$

40.39 (12)

$129.72(14)$

$166.74(14)$

$151.53(15)$

40.42 (16)

$123.28(15)$

$104.47(15)$

$117.11(15)$

$152.72(15)$

$67.74(16)$

$40.00(16)$

$40.69(16)$

$67.98(16)$

$160.10(15)$

$105.78(15)$

$119.80(14)$

68.08 (16)

$\mathrm{C} 8 \mathrm{~B}-\mathrm{Fe} 1 \mathrm{~B}-\mathrm{C} 9 \mathrm{~B}$

$\mathrm{C} 8 \mathrm{~B}-\mathrm{Fe} 1 \mathrm{~B}-\mathrm{C} 10 \mathrm{~B}$

$\mathrm{C} 9 \mathrm{~B}-\mathrm{Fe} 1 \mathrm{~B}-\mathrm{C} 2 \mathrm{~B}$

$\mathrm{C} 9 \mathrm{~B}-\mathrm{Fe} 1 \mathrm{~B}-\mathrm{C} 3 \mathrm{~B}$

$\mathrm{C} 9 \mathrm{~B}-\mathrm{Fe} 1 \mathrm{~B}-\mathrm{C} 4 \mathrm{~B}$

$\mathrm{C} 9 \mathrm{~B}-\mathrm{Fe} 1 \mathrm{~B}-\mathrm{C} 6 \mathrm{~B}$

C9B-Fe1B-C7B

$\mathrm{C} 9 \mathrm{~B}-\mathrm{Fe} 1 \mathrm{~B}-\mathrm{C} 10 \mathrm{~B}$

$\mathrm{C} 10 \mathrm{~B}-\mathrm{Fe} 1 \mathrm{~B}-\mathrm{C} 4 \mathrm{~B}$

$\mathrm{C} 15 \mathrm{~B}-\mathrm{Fe} 2 \mathrm{~B}-\mathrm{C} 16 \mathrm{~B}$

$\mathrm{C} 15 \mathrm{~B}-\mathrm{Fe} 2 \mathrm{~B}-\mathrm{C} 17 \mathrm{~B}$

$\mathrm{C} 15 \mathrm{~B}-\mathrm{Fe} 2 \mathrm{~B}-\mathrm{C} 18 \mathrm{~B}$

$\mathrm{C} 15 \mathrm{~B}-\mathrm{Fe} 2 \mathrm{~B}-\mathrm{C} 19 \mathrm{~B}$

$\mathrm{C} 15 \mathrm{~B}-\mathrm{Fe} 2 \mathrm{~B}-\mathrm{C} 20 \mathrm{~B}$

$\mathrm{C} 15 \mathrm{~B}-\mathrm{Fe} 2 \mathrm{~B}-\mathrm{C} 21 \mathrm{~B}$

$\mathrm{C} 15 \mathrm{~B}-\mathrm{Fe} 2 \mathrm{~B}-\mathrm{C} 22 \mathrm{~B}$

$\mathrm{C} 15 \mathrm{~B}-\mathrm{Fe} 2 \mathrm{~B}-\mathrm{C} 23 \mathrm{~B}$

$\mathrm{C} 15 \mathrm{~B}-\mathrm{Fe} 2 \mathrm{~B}-\mathrm{C} 24 \mathrm{~B}$

$\mathrm{C} 16 \mathrm{~B}-\mathrm{Fe} 2 \mathrm{~B}-\mathrm{C} 20 \mathrm{~B}$

$\mathrm{C} 16 \mathrm{~B}-\mathrm{Fe} 2 \mathrm{~B}-\mathrm{C} 24 \mathrm{~B}$

$\mathrm{C} 17 \mathrm{~B}-\mathrm{Fe} 2 \mathrm{~B}-\mathrm{C} 16 \mathrm{~B}$

C17B-Fe2B-C20B

$\mathrm{C} 17 \mathrm{~B}-\mathrm{Fe} 2 \mathrm{~B}-\mathrm{C} 24 \mathrm{~B}$

$\mathrm{C} 18 \mathrm{~B}-\mathrm{Fe} 2 \mathrm{~B}-\mathrm{C} 16 \mathrm{~B}$

$\mathrm{C} 18 \mathrm{~B}-\mathrm{Fe} 2 \mathrm{~B}-\mathrm{C} 17 \mathrm{~B}$

$\mathrm{C} 18 \mathrm{~B}-\mathrm{Fe} 2 \mathrm{~B}-\mathrm{C} 19 \mathrm{~B}$

$\mathrm{C} 18 \mathrm{~B}-\mathrm{Fe} 2 \mathrm{~B}-\mathrm{C} 20 \mathrm{~B}$

$\mathrm{C} 18 \mathrm{~B}-\mathrm{Fe} 2 \mathrm{~B}-\mathrm{C} 21 \mathrm{~B}$

$\mathrm{C} 18 \mathrm{~B}-\mathrm{Fe} 2 \mathrm{~B}-\mathrm{C} 22 \mathrm{~B}$

$\mathrm{C} 18 \mathrm{~B}-\mathrm{Fe} 2 \mathrm{~B}-\mathrm{C} 23 \mathrm{~B}$

$\mathrm{C} 18 \mathrm{~B}-\mathrm{Fe} 2 \mathrm{~B}-\mathrm{C} 24 \mathrm{~B}$

$\mathrm{C} 19 \mathrm{~B}-\mathrm{Fe} 2 \mathrm{~B}-\mathrm{C} 16 \mathrm{~B}$

$\mathrm{C} 19 \mathrm{~B}-\mathrm{Fe} 2 \mathrm{~B}-\mathrm{C} 17 \mathrm{~B}$

$\mathrm{C} 19 \mathrm{~B}-\mathrm{Fe} 2 \mathrm{~B}-\mathrm{C} 20 \mathrm{~B}$

$\mathrm{C} 19 \mathrm{~B}-\mathrm{Fe} 2 \mathrm{~B}-\mathrm{C} 22 \mathrm{~B}$

$\mathrm{C} 19 \mathrm{~B}-\mathrm{Fe} 2 \mathrm{~B}-\mathrm{C} 24 \mathrm{~B}$

$\mathrm{C} 20 \mathrm{~B}-\mathrm{Fe} 2 \mathrm{~B}-\mathrm{C} 24 \mathrm{~B}$

$\mathrm{C} 21 \mathrm{~B}-\mathrm{Fe} 2 \mathrm{~B}-\mathrm{C} 16 \mathrm{~B}$

$\mathrm{C} 21 \mathrm{~B}-\mathrm{Fe} 2 \mathrm{~B}-\mathrm{C} 17 \mathrm{~B}$

$\mathrm{C} 21 \mathrm{~B}-\mathrm{Fe} 2 \mathrm{~B}-\mathrm{C} 19 \mathrm{~B}$

$\mathrm{C} 21 \mathrm{~B}-\mathrm{Fe} 2 \mathrm{~B}-\mathrm{C} 20 \mathrm{~B}$

$\mathrm{C} 21 \mathrm{~B}-\mathrm{Fe} 2 \mathrm{~B}-\mathrm{C} 22 \mathrm{~B}$

$\mathrm{C} 21 \mathrm{~B}-\mathrm{Fe} 2 \mathrm{~B}-\mathrm{C} 23 \mathrm{~B}$

$\mathrm{C} 21 \mathrm{~B}-\mathrm{Fe} 2 \mathrm{~B}-\mathrm{C} 24 \mathrm{~B}$

$\mathrm{C} 22 \mathrm{~B}-\mathrm{Fe} 2 \mathrm{~B}-\mathrm{C} 16 \mathrm{~B}$

$\mathrm{C} 22 \mathrm{~B}-\mathrm{Fe} 2 \mathrm{~B}-\mathrm{C} 17 \mathrm{~B}$

$\mathrm{C} 22 \mathrm{~B}-\mathrm{Fe} 2 \mathrm{~B}-\mathrm{C} 20 \mathrm{~B}$

$\mathrm{C} 22 \mathrm{~B}-\mathrm{Fe} 2 \mathrm{~B}-\mathrm{C} 24 \mathrm{~B}$
40.70 (19)

$67.65(18)$

$160.57(15)$

$122.65(16)$

$105.26(16)$

$67.28(16)$

68.87 (17)

$39.68(17)$

$125.04(15)$

$41.72(12)$

$69.40(12)$

$69.14(12)$

$41.36(11)$

$109.69(12)$

$122.62(14)$

$156.63(16)$

$162.42(17)$

$126.22(14)$

$124.48(13)$

$162.13(13)$

41.02 (12)

159.35 (14)

$156.64(14)$

68.79 (13)

40.32 (14)

$40.50(12)$

160.04 (14)

157.09 (14)

$120.62(14)$

$106.68(15)$

$123.43(15)$

69.34 (13)

68.36 (13)

$125.46(13)$

158.33 (15)

$110.46(15)$

40.13 (14)

105.89 (16)

$121.21(15)$

160.47 (14)

40.71 (15)

40.83 (15)

67.92 (18)

68.01 (17)

118.57 (16)

$103.58(15)$

68.73 (14)

68.59 (17) 


\begin{tabular}{|c|c|}
\hline $\mathrm{C} 23 \mathrm{~A}-\mathrm{Fe} 2 \mathrm{~A}-\mathrm{C} 21 \mathrm{~A}$ & $67.97(16)$ \\
\hline $\mathrm{C} 23 \mathrm{~A}-\mathrm{Fe} 2 \mathrm{~A}-\mathrm{C} 24 \mathrm{~A}$ & $40.67(16)$ \\
\hline $\mathrm{C} 24 \mathrm{~A}-\mathrm{Fe} 2 \mathrm{~A}-\mathrm{C} 18 \mathrm{~A}$ & $126.61(15)$ \\
\hline $\mathrm{C} 24 \mathrm{~A}-\mathrm{Fe} 2 \mathrm{~A}-\mathrm{C} 19 \mathrm{~A}$ & $110.37(15)$ \\
\hline $\mathrm{C} 24 \mathrm{~A}-\mathrm{Fe} 2 \mathrm{~A}-\mathrm{C} 20 \mathrm{~A}$ & $40.12(16)$ \\
\hline $\mathrm{C} 24 \mathrm{~A}-\mathrm{Fe} 2 \mathrm{~A}-\mathrm{C} 21 \mathrm{~A}$ & $67.70(16)$ \\
\hline $\mathrm{C} 1 \mathrm{~A}-\mathrm{P} 1 \mathrm{~A}-\mathrm{S} 1 \mathrm{~A}$ & $116.13(10)$ \\
\hline $\mathrm{C} 1 \mathrm{~A}-\mathrm{P} 1 \mathrm{~A}-\mathrm{C} 34 \mathrm{~A}$ & $103.68(13)$ \\
\hline $\mathrm{C} 15 \mathrm{~A}-\mathrm{P} 1 \mathrm{~A}-\mathrm{S} 1 \mathrm{~A}$ & $117.58(10)$ \\
\hline $\mathrm{C} 15 \mathrm{~A}-\mathrm{P} 1 \mathrm{~A}-\mathrm{C} 1 \mathrm{~A}$ & $102.69(13)$ \\
\hline $\mathrm{C} 15 \mathrm{~A}-\mathrm{P} 1 \mathrm{~A}-\mathrm{C} 34 \mathrm{~A}$ & $103.07(13)$ \\
\hline $\mathrm{C} 34 \mathrm{~A}-\mathrm{P} 1 \mathrm{~A}-\mathrm{S} 1 \mathrm{~A}$ & $111.94(9)$ \\
\hline $\mathrm{P} 1 \mathrm{~A}-\mathrm{C} 1 \mathrm{~A}-\mathrm{Fe} 1 \mathrm{~A}$ & $130.23(15)$ \\
\hline $\mathrm{C} 2 \mathrm{~A}-\mathrm{C} 1 \mathrm{~A}-\mathrm{Fe} 1 \mathrm{~A}$ & $70.39(16)$ \\
\hline $\mathrm{C} 2 \mathrm{~A}-\mathrm{C} 1 \mathrm{~A}-\mathrm{P} 1 \mathrm{~A}$ & $129.3(2)$ \\
\hline $\mathrm{C} 5 \mathrm{~A}-\mathrm{C} 1 \mathrm{~A}-\mathrm{Fe} 1 \mathrm{~A}$ & $70.26(17)$ \\
\hline $\mathrm{C} 5 \mathrm{~A}-\mathrm{C} 1 \mathrm{~A}-\mathrm{P} 1 \mathrm{~A}$ & $122.6(2)$ \\
\hline $\mathrm{C} 5 \mathrm{~A}-\mathrm{C} 1 \mathrm{~A}-\mathrm{C} 2 \mathrm{~A}$ & $107.8(2)$ \\
\hline $\mathrm{C} 1 \mathrm{~A}-\mathrm{C} 2 \mathrm{~A}-\mathrm{Fe} 1 \mathrm{~A}$ & $67.73(16)$ \\
\hline $\mathrm{C} 1 \mathrm{~A}-\mathrm{C} 2 \mathrm{~A}-\mathrm{C} 11 \mathrm{~A}$ & $127.6(3)$ \\
\hline $\mathrm{C} 3 \mathrm{~A}-\mathrm{C} 2 \mathrm{~A}-\mathrm{Fe} 1 \mathrm{~A}$ & $69.43(17)$ \\
\hline $\mathrm{C} 3 \mathrm{~A}-\mathrm{C} 2 \mathrm{~A}-\mathrm{C} 1 \mathrm{~A}$ & $106.6(3)$ \\
\hline $\mathrm{C} 3 \mathrm{~A}-\mathrm{C} 2 \mathrm{~A}-\mathrm{C} 11 \mathrm{~A}$ & $125.8(3)$ \\
\hline $\mathrm{C} 11 \mathrm{~A}-\mathrm{C} 2 \mathrm{~A}-\mathrm{Fe} 1 \mathrm{~A}$ & $126.0(2)$ \\
\hline $\mathrm{Fe} 1 \mathrm{~A}-\mathrm{C} 3 \mathrm{~A}-\mathrm{H} 3 \mathrm{~A}$ & 126.0 \\
\hline $\mathrm{C} 2 \mathrm{~A}-\mathrm{C} 3 \mathrm{~A}-\mathrm{Fe} 1 \mathrm{~A}$ & $69.65(16)$ \\
\hline $\mathrm{C} 2 \mathrm{~A}-\mathrm{C} 3 \mathrm{~A}-\mathrm{H} 3 \mathrm{~A}$ & 125.5 \\
\hline $\mathrm{C} 4 \mathrm{~A}-\mathrm{C} 3 \mathrm{~A}-\mathrm{Fe} 1 \mathrm{~A}$ & $70.39(18)$ \\
\hline $\mathrm{C} 4 \mathrm{~A}-\mathrm{C} 3 \mathrm{~A}-\mathrm{C} 2 \mathrm{~A}$ & $109.0(3)$ \\
\hline $\mathrm{C} 4 \mathrm{~A}-\mathrm{C} 3 \mathrm{~A}-\mathrm{H} 3 \mathrm{~A}$ & 125.5 \\
\hline $\mathrm{Fe} 1 \mathrm{~A}-\mathrm{C} 4 \mathrm{~A}-\mathrm{H} 4 \mathrm{~A}$ & 127.6 \\
\hline $\mathrm{C} 3 \mathrm{~A}-\mathrm{C} 4 \mathrm{~A}-\mathrm{Fe} 1 \mathrm{~A}$ & $69.25(17)$ \\
\hline $\mathrm{C} 3 \mathrm{~A}-\mathrm{C} 4 \mathrm{~A}-\mathrm{H} 4 \mathrm{~A}$ & 125.8 \\
\hline $\mathrm{C} 3 \mathrm{~A}-\mathrm{C} 4 \mathrm{~A}-\mathrm{C} 5 \mathrm{~A}$ & $108.4(3)$ \\
\hline $\mathrm{C} 5 \mathrm{~A}-\mathrm{C} 4 \mathrm{~A}-\mathrm{Fe} 1 \mathrm{~A}$ & $68.93(17)$ \\
\hline $\mathrm{C} 5 \mathrm{~A}-\mathrm{C} 4 \mathrm{~A}-\mathrm{H} 4 \mathrm{~A}$ & 125.8 \\
\hline $\mathrm{Fe} 1 \mathrm{~A}-\mathrm{C} 5 \mathrm{~A}-\mathrm{H} 5 \mathrm{~A}$ & 126.7 \\
\hline $\mathrm{C} 1 \mathrm{~A}-\mathrm{C} 5 \mathrm{~A}-\mathrm{Fe} 1 \mathrm{~A}$ & $68.32(17)$ \\
\hline $\mathrm{C} 1 \mathrm{~A}-\mathrm{C} 5 \mathrm{~A}-\mathrm{H} 5 \mathrm{~A}$ & 125.9 \\
\hline $\mathrm{C} 4 \mathrm{~A}-\mathrm{C} 5 \mathrm{~A}-\mathrm{Fe} 1 \mathrm{~A}$ & $70.62(18)$ \\
\hline $\mathrm{C} 4 \mathrm{~A}-\mathrm{C} 5 \mathrm{~A}-\mathrm{C} 1 \mathrm{~A}$ & $108.2(3)$ \\
\hline $\mathrm{C} 4 \mathrm{~A}-\mathrm{C} 5 \mathrm{~A}-\mathrm{H} 5 \mathrm{~A}$ & 125.9 \\
\hline $\mathrm{Fe} 1 \mathrm{~A}-\mathrm{C} 6 \mathrm{~A}-\mathrm{H} 6 \mathrm{~A}$ & 125.8 \\
\hline $\mathrm{C} 7 \mathrm{~A}-\mathrm{C} 6 \mathrm{~A}-\mathrm{Fe} 1 \mathrm{~A}$ & $69.62(19)$ \\
\hline $\mathrm{C} 7 \mathrm{~A}-\mathrm{C} 6 \mathrm{~A}-\mathrm{H} 6 \mathrm{~A}$ & 125.8 \\
\hline $\mathrm{C} 7 \mathrm{~A}-\mathrm{C} 6 \mathrm{~A}-\mathrm{C} 10 \mathrm{~A}$ & $108.4(3)$ \\
\hline $\mathrm{C} 10 \mathrm{~A}-\mathrm{C} 6 \mathrm{~A}-\mathrm{Fe} 1 \mathrm{~A}$ & $70.40(19)$ \\
\hline & \\
\hline
\end{tabular}

$\begin{array}{ll}\text { C23B-Fe2B-C16B } & 154.27(16) \\ \text { C23B-Fe2B-C17B } & 119.17(14) \\ \text { C23B-Fe2B-C19B } & 124.60(17) \\ \text { C23B-Fe2B-C20B } & 68.09(14) \\ \text { C23B-Fe2B-C22B } & 40.39(18) \\ \text { C23B-Fe2B-C24B } & 40.85(15) \\ \text { C1B-P1B-S1B } & 116.14(10) \\ \text { C1B-P1B-C15B } & 102.76(13) \\ \text { C1B-P1B-C34B } & 103.49(13) \\ \text { C15B-P1B-S1B } & 116.81(10) \\ \text { C15B-P1B-C34B } & 104.50(13) \\ \text { C34B-P1B-S1B } & 111.60(10) \\ \text { P1B-C1B-Fe1B } & 131.42(15) \\ \text { C2B-C1B-Fe1B } & 69.70(16) \\ \text { C2B-C1B-P1B } & 129.7(2) \\ \text { C5B-C1B-Fe1B } & 69.83(17) \\ \text { C5B-C1B-P1B } & 122.5(2) \\ \text { C5B-C1B-C2B } & 107.4(3) \\ \text { C1B-C2B-Fe1B } & 68.35(16) \\ \text { C1B-C2B-C11B } & 128.0(3) \\ \text { C3B-C2B-Fe1B } & 69.48(18) \\ \text { C3B-C2B-C1B } & 106.7(3) \\ \text { C3B-C2B-C11B } & 125.3(3) \\ \text { C11B-C2B-Fe1B } & 124.9(2) \\ \text { Fe1B-C3B-H3B } & 126.1 \\ \text { C2B-C3B-Fe1B } & 69.37(17) \\ \text { C2B-C3B-H3B } & 125.4 \\ \text { C4B-C3B-Fe1B } & 70.76(18) \\ \text { C4B-C3B-C2B } & 109.3(3) \\ \text { C4B-C3B-H3B } & 125.4 \\ \text { Fe1B-C4B-H4B } & 127.9 \\ \text { C3B-C4B-Fe1B } & 69.09(18) \\ \text { C3B-C4B-H4B } & 125.8 \\ \text { C3B-C4B-C5B } & 108.5(3) \\ \text { C5B-C4B-Fe1B } & 68.79(17) \\ \text { C5B-C4B-H4B } & 125.8 \\ \text { Fe1B-C5B-H5B } & 126.4 \\ \text { C1B-C5B-Fe1B } & 68.62(16) \\ \text { C1B-C5B-H5B } & 125.9 \\ \text { C4B-C5B-Fe1B } & 70.72(18) \\ \text { C4B-C5B-C1B } & 108.3(3) \\ \text { C4B-C5B-H5B } & 125.9 \\ \text { Fe1B-C6B-H6B } & 126.1 \\ \text { C7B-C6B-Fe1B } & 70.0(2) \\ \text { C7B-C6B-H6B } & 125.1 \\ \text { C7B-C6B-C10B } & \\ \text { C10B-C6B-Fe1B } & \\ \text { C10B-C6B-H6B } & \\ & \end{array}$


Fe1A-C7A-H7A

C6A-C7A-Fe1A

C6A-C7A-H7A

$\mathrm{C} 6 \mathrm{~A}-\mathrm{C} 7 \mathrm{~A}-\mathrm{C} 8 \mathrm{~A}$

$\mathrm{C} 8 \mathrm{~A}-\mathrm{C} 7 \mathrm{~A}-\mathrm{Fe} 1 \mathrm{~A}$

C8A-C7A-H7A

$\mathrm{Fe} 1 \mathrm{~A}-\mathrm{C} 8 \mathrm{~A}-\mathrm{H} 8 \mathrm{~A}$

$\mathrm{C} 7 \mathrm{~A}-\mathrm{C} 8 \mathrm{~A}-\mathrm{Fe} 1 \mathrm{~A}$

C7A-C8A-H8A

$\mathrm{C} 9 \mathrm{~A}-\mathrm{C} 8 \mathrm{~A}-\mathrm{Fe} 1 \mathrm{~A}$

C9A $-\mathrm{C} 8 \mathrm{~A}-\mathrm{C} 7 \mathrm{~A}$

C9A-C8A-H8A

Fe1A-C9A-H9A

$\mathrm{C} 8 \mathrm{~A}-\mathrm{C} 9 \mathrm{~A}-\mathrm{Fe} 1 \mathrm{~A}$

$\mathrm{C} 8 \mathrm{~A}-\mathrm{C} 9 \mathrm{~A}-\mathrm{H} 9 \mathrm{~A}$

$\mathrm{C} 8 \mathrm{~A}-\mathrm{C} 9 \mathrm{~A}-\mathrm{C} 10 \mathrm{~A}$

C10A-C9A-Fe1A

C10A-C9A-H9A

Fe1A-C10A-H10A

C6A-C10A-Fe1A

C6A-C10A-C9A

C6A-C10A-H10A

$\mathrm{C} 9 \mathrm{~A}-\mathrm{C} 10 \mathrm{~A}-\mathrm{Fe} 1 \mathrm{~A}$

C9A-C10A-H10A

$\mathrm{C} 2 \mathrm{~A}-\mathrm{C} 11 \mathrm{~A}-\mathrm{H} 11 \mathrm{~A}$

$\mathrm{C} 12 \mathrm{~A}-\mathrm{C} 11 \mathrm{~A}-\mathrm{C} 2 \mathrm{~A}$

C12A-C11A-H11A

C11A-C12A-H12A

C11A-C12A-H12B

H12A-C12A-H12B

$\mathrm{P} 1 \mathrm{~A}-\mathrm{C} 15 \mathrm{~A}-\mathrm{Fe} 2 \mathrm{~A}$

$\mathrm{C} 16 \mathrm{~A}-\mathrm{C} 15 \mathrm{~A}-\mathrm{Fe} 2 \mathrm{~A}$

C16A-C15A-P1A

$\mathrm{C} 19 \mathrm{~A}-\mathrm{C} 15 \mathrm{~A}-\mathrm{Fe} 2 \mathrm{~A}$

C19A-C15A-P1A

$\mathrm{C} 19 \mathrm{~A}-\mathrm{C} 15 \mathrm{~A}-\mathrm{C} 16 \mathrm{~A}$

$\mathrm{C} 15 \mathrm{~A}-\mathrm{C} 16 \mathrm{~A}-\mathrm{Fe} 2 \mathrm{~A}$

$\mathrm{C} 15 \mathrm{~A}-\mathrm{C} 16 \mathrm{~A}-\mathrm{C} 25 \mathrm{~A}$

$\mathrm{C} 17 \mathrm{~A}-\mathrm{C} 16 \mathrm{~A}-\mathrm{Fe} 2 \mathrm{~A}$

$\mathrm{C} 17 \mathrm{~A}-\mathrm{C} 16 \mathrm{~A}-\mathrm{C} 15 \mathrm{~A}$

$\mathrm{C} 17 \mathrm{~A}-\mathrm{C} 16 \mathrm{~A}-\mathrm{C} 25 \mathrm{~A}$

$\mathrm{C} 25 \mathrm{~A}-\mathrm{C} 16 \mathrm{~A}-\mathrm{Fe} 2 \mathrm{~A}$

Fe2A-C17A-H17A

C16A-C17A-Fe2A

C16A-C17A-H17A

$\mathrm{C} 18 \mathrm{~A}-\mathrm{C} 17 \mathrm{~A}-\mathrm{Fe} 2 \mathrm{~A}$

$\mathrm{C} 18 \mathrm{~A}-\mathrm{C} 17 \mathrm{~A}-\mathrm{C} 16 \mathrm{~A}$

$\mathrm{C} 18 \mathrm{~A}-\mathrm{C} 17 \mathrm{~A}-\mathrm{H} 17 \mathrm{~A}$
125.8

69.9 (2)

126.1

107.9 (4)

69.8 (2)

126.1

125.9

69.53 (19)

125.8

70.3 (2)

$108.3(3)$

125.8

126.3

69.3 (2)

126.1

107.8 (3)

69.9 (2)

126.1

126.6

69.08 (19)

107.5 (3)

126.2

69.6 (2)

126.2

117.7

124.6 (3)

117.7

120.0

120.0

120.0

133.21 (15)

69.79 (16)

127.9 (2)

70.06 (16)

123.9 (2)

107.4 (3)

68.61 (16)

126.4 (3)

69.41 (17)

107.0 (3)

126.7 (3)

126.7 (2)

126.1

69.81 (17)

125.4

70.33 (19)

109.3 (3)

125.4
$\mathrm{Fe} 1 \mathrm{~B}-\mathrm{C} 7 \mathrm{~B}-\mathrm{H} 7 \mathrm{~B}$

$\mathrm{C} 6 \mathrm{~B}-\mathrm{C} 7 \mathrm{~B}-\mathrm{Fe} 1 \mathrm{~B}$

$\mathrm{C} 6 \mathrm{~B}-\mathrm{C} 7 \mathrm{~B}-\mathrm{H} 7 \mathrm{~B}$

$\mathrm{C} 6 \mathrm{~B}-\mathrm{C} 7 \mathrm{~B}-\mathrm{C} 8 \mathrm{~B}$

$\mathrm{C} 8 \mathrm{~B}-\mathrm{C} 7 \mathrm{~B}-\mathrm{Fe} 1 \mathrm{~B}$

$\mathrm{C} 8 \mathrm{~B}-\mathrm{C} 7 \mathrm{~B}-\mathrm{H} 7 \mathrm{~B}$

$\mathrm{Fe} 1 \mathrm{~B}-\mathrm{C} 8 \mathrm{~B}-\mathrm{H} 8 \mathrm{~B}$

$\mathrm{C} 7 \mathrm{~B}-\mathrm{C} 8 \mathrm{~B}-\mathrm{Fe} 1 \mathrm{~B}$

$\mathrm{C} 7 \mathrm{~B}-\mathrm{C} 8 \mathrm{~B}-\mathrm{H} 8 \mathrm{~B}$

$\mathrm{C} 9 \mathrm{~B}-\mathrm{C} 8 \mathrm{~B}-\mathrm{Fe} 1 \mathrm{~B}$

$\mathrm{C} 9 \mathrm{~B}-\mathrm{C} 8 \mathrm{~B}-\mathrm{C} 7 \mathrm{~B}$

$\mathrm{C} 9 \mathrm{~B}-\mathrm{C} 8 \mathrm{~B}-\mathrm{H} 8 \mathrm{~B}$

$\mathrm{Fe} 1 \mathrm{~B}-\mathrm{C} 9 \mathrm{~B}-\mathrm{H} 9 \mathrm{~B}$

$\mathrm{C} 8 \mathrm{~B}-\mathrm{C} 9 \mathrm{~B}-\mathrm{Fe} 1 \mathrm{~B}$

$\mathrm{C} 8 \mathrm{~B}-\mathrm{C} 9 \mathrm{~B}-\mathrm{H} 9 \mathrm{~B}$

$\mathrm{C} 10 \mathrm{~B}-\mathrm{C} 9 \mathrm{~B}-\mathrm{Fe} 1 \mathrm{~B}$

$\mathrm{C} 10 \mathrm{~B}-\mathrm{C} 9 \mathrm{~B}-\mathrm{C} 8 \mathrm{~B}$

$\mathrm{C} 10 \mathrm{~B}-\mathrm{C} 9 \mathrm{~B}-\mathrm{H} 9 \mathrm{~B}$

$\mathrm{Fe} 1 \mathrm{~B}-\mathrm{C} 10 \mathrm{~B}-\mathrm{H} 10 \mathrm{~B}$

$\mathrm{C} 6 \mathrm{~B}-\mathrm{C} 10 \mathrm{~B}-\mathrm{Fe} 1 \mathrm{~B}$

$\mathrm{C} 6 \mathrm{~B}-\mathrm{C} 10 \mathrm{~B}-\mathrm{H} 10 \mathrm{~B}$

$\mathrm{C} 9 \mathrm{~B}-\mathrm{C} 10 \mathrm{~B}-\mathrm{Fe} 1 \mathrm{~B}$

$\mathrm{C} 9 \mathrm{~B}-\mathrm{C} 10 \mathrm{~B}-\mathrm{C} 6 \mathrm{~B}$

$\mathrm{C} 9 \mathrm{~B}-\mathrm{C} 10 \mathrm{~B}-\mathrm{H} 10 \mathrm{~B}$

$\mathrm{C} 2 \mathrm{~B}-\mathrm{C} 11 \mathrm{~B}-\mathrm{H} 11 \mathrm{~B}$

$\mathrm{C} 12 \mathrm{~B}-\mathrm{C} 11 \mathrm{~B}-\mathrm{C} 2 \mathrm{~B}$

C12B-C11B-H11B

$\mathrm{C} 11 \mathrm{~B}-\mathrm{C} 12 \mathrm{~B}-\mathrm{H} 12 \mathrm{C}$

$\mathrm{C} 11 \mathrm{~B}-\mathrm{C} 12 \mathrm{~B}-\mathrm{H} 12 \mathrm{D}$

$\mathrm{H} 12 \mathrm{C}-\mathrm{C} 12 \mathrm{~B}-\mathrm{H} 12 \mathrm{D}$

$\mathrm{P} 1 \mathrm{~B}-\mathrm{C} 15 \mathrm{~B}-\mathrm{Fe} 2 \mathrm{~B}$

$\mathrm{C} 16 \mathrm{~B}-\mathrm{C} 15 \mathrm{~B}-\mathrm{Fe} 2 \mathrm{~B}$

$\mathrm{C} 16 \mathrm{~B}-\mathrm{C} 15 \mathrm{~B}-\mathrm{P} 1 \mathrm{~B}$

$\mathrm{C} 19 \mathrm{~B}-\mathrm{C} 15 \mathrm{~B}-\mathrm{Fe} 2 \mathrm{~B}$

$\mathrm{C} 19 \mathrm{~B}-\mathrm{C} 15 \mathrm{~B}-\mathrm{P} 1 \mathrm{~B}$

$\mathrm{C} 19 \mathrm{~B}-\mathrm{C} 15 \mathrm{~B}-\mathrm{C} 16 \mathrm{~B}$

$\mathrm{C} 15 \mathrm{~B}-\mathrm{C} 16 \mathrm{~B}-\mathrm{Fe} 2 \mathrm{~B}$

$\mathrm{C} 15 \mathrm{~B}-\mathrm{C} 16 \mathrm{~B}-\mathrm{C} 25 \mathrm{~B}$

$\mathrm{C} 17 \mathrm{~B}-\mathrm{C} 16 \mathrm{~B}-\mathrm{Fe} 2 \mathrm{~B}$

$\mathrm{C} 17 \mathrm{~B}-\mathrm{C} 16 \mathrm{~B}-\mathrm{C} 15 \mathrm{~B}$

$\mathrm{C} 17 \mathrm{~B}-\mathrm{C} 16 \mathrm{~B}-\mathrm{C} 25 \mathrm{~B}$

$\mathrm{C} 25 \mathrm{~B}-\mathrm{C} 16 \mathrm{~B}-\mathrm{Fe} 2 \mathrm{~B}$

$\mathrm{Fe} 2 \mathrm{~B}-\mathrm{C} 17 \mathrm{~B}-\mathrm{H} 17 \mathrm{~B}$

$\mathrm{C} 16 \mathrm{~B}-\mathrm{C} 17 \mathrm{~B}-\mathrm{Fe} 2 \mathrm{~B}$

$\mathrm{C} 16 \mathrm{~B}-\mathrm{C} 17 \mathrm{~B}-\mathrm{H} 17 \mathrm{~B}$

$\mathrm{C} 18 \mathrm{~B}-\mathrm{C} 17 \mathrm{~B}-\mathrm{Fe} 2 \mathrm{~B}$

$\mathrm{C} 18 \mathrm{~B}-\mathrm{C} 17 \mathrm{~B}-\mathrm{C} 16 \mathrm{~B}$

$\mathrm{C} 18 \mathrm{~B}-\mathrm{C} 17 \mathrm{~B}-\mathrm{H} 17 \mathrm{~B}$
125.9

$70.2(2)$

126.8

106.3 (4)

68.7 (2)

126.8

125.6

69.7 (2)

126.3

$70.0(2)$

107.4 (4)

126.3

125.6

69.3 (2)

125.8

70.9 (2)

108.4 (4)

125.8

126.6

69.5 (2)

126.0

69.5 (2)

108.1 (4)

126.0

117.4

125.1 (3)

117.4

120.0

120.0

120.0

129.25 (15)

69.40 (16)

128.8 (2)

69.51 (17)

124.2 (2)

106.9 (3)

68.88 (16)

126.2 (3)

69.44 (17)

107.2 (3)

126.6 (3)

126.2 (3)

126.7

69.53 (17)

125.7

69.68 (18)

108.7 (3)

125.7 


\begin{tabular}{|c|c|}
\hline $\mathrm{Fe} 2 \mathrm{~A}-\mathrm{C} 18 \mathrm{~A}-\mathrm{H} 18 \mathrm{~A}$ & 126.9 \\
\hline $\mathrm{C} 17 \mathrm{~A}-\mathrm{C} 18 \mathrm{~A}-\mathrm{Fe} 2 \mathrm{~A}$ & 69.17 (19) \\
\hline $\mathrm{C} 17 \mathrm{~A}-\mathrm{C} 18 \mathrm{~A}-\mathrm{H} 18 \mathrm{~A}$ & 126.0 \\
\hline $\mathrm{C} 19 \mathrm{~A}-\mathrm{C} 18 \mathrm{~A}-\mathrm{Fe} 2 \mathrm{~A}$ & $69.56(18)$ \\
\hline $\mathrm{C} 19 \mathrm{~A}-\mathrm{C} 18 \mathrm{~A}-\mathrm{C} 17 \mathrm{~A}$ & $108.1(3)$ \\
\hline $\mathrm{C} 19 \mathrm{~A}-\mathrm{C} 18 \mathrm{~A}-\mathrm{H} 18 \mathrm{~A}$ & 126.0 \\
\hline $\mathrm{Fe} 2 \mathrm{~A}-\mathrm{C} 19 \mathrm{~A}-\mathrm{H} 19 \mathrm{~A}$ & 127.0 \\
\hline $\mathrm{C} 15 \mathrm{~A}-\mathrm{C} 19 \mathrm{~A}-\mathrm{Fe} 2 \mathrm{~A}$ & $68.61(16)$ \\
\hline $\mathrm{C} 15 \mathrm{~A}-\mathrm{C} 19 \mathrm{~A}-\mathrm{H} 19 \mathrm{~A}$ & 125.9 \\
\hline $\mathrm{C} 18 \mathrm{~A}-\mathrm{C} 19 \mathrm{~A}-\mathrm{Fe} 2 \mathrm{~A}$ & $70.05(18)$ \\
\hline $\mathrm{C} 18 \mathrm{~A}-\mathrm{C} 19 \mathrm{~A}-\mathrm{C} 15 \mathrm{~A}$ & $108.2(3)$ \\
\hline $\mathrm{C} 18 \mathrm{~A}-\mathrm{C} 19 \mathrm{~A}-\mathrm{H} 19 \mathrm{~A}$ & 125.9 \\
\hline $\mathrm{Fe} 2 \mathrm{~A}-\mathrm{C} 20 \mathrm{~A}-\mathrm{H} 20 \mathrm{~A}$ & 126.3 \\
\hline $\mathrm{C} 21 \mathrm{~A}-\mathrm{C} 20 \mathrm{~A}-\mathrm{Fe} 2 \mathrm{~A}$ & $69.6(2)$ \\
\hline $\mathrm{C} 21 \mathrm{~A}-\mathrm{C} 20 \mathrm{~A}-\mathrm{H} 20 \mathrm{~A}$ & 126.2 \\
\hline $\mathrm{C} 24 \mathrm{~A}-\mathrm{C} 20 \mathrm{~A}-\mathrm{Fe} 2 \mathrm{~A}$ & $69.5(2)$ \\
\hline $\mathrm{C} 24 \mathrm{~A}-\mathrm{C} 20 \mathrm{~A}-\mathrm{H} 20 \mathrm{~A}$ & 126.2 \\
\hline $\mathrm{C} 24 \mathrm{~A}-\mathrm{C} 20 \mathrm{~A}-\mathrm{C} 21 \mathrm{~A}$ & $107.7(4)$ \\
\hline $\mathrm{Fe} 2 \mathrm{~A}-\mathrm{C} 21 \mathrm{~A}-\mathrm{H} 21 \mathrm{~A}$ & 126.2 \\
\hline $\mathrm{C} 20 \mathrm{~A}-\mathrm{C} 21 \mathrm{~A}-\mathrm{Fe} 2 \mathrm{~A}$ & $70.0(2)$ \\
\hline $\mathrm{C} 20 \mathrm{~A}-\mathrm{C} 21 \mathrm{~A}-\mathrm{H} 21 \mathrm{~A}$ & 125.9 \\
\hline $\mathrm{C} 22 \mathrm{~A}-\mathrm{C} 21 \mathrm{~A}-\mathrm{Fe} 2 \mathrm{~A}$ & $69.4(2)$ \\
\hline $\mathrm{C} 22 \mathrm{~A}-\mathrm{C} 21 \mathrm{~A}-\mathrm{C} 20 \mathrm{~A}$ & $108.1(3)$ \\
\hline $\mathrm{C} 22 \mathrm{~A}-\mathrm{C} 21 \mathrm{~A}-\mathrm{H} 21 \mathrm{~A}$ & 125.9 \\
\hline $\mathrm{Fe} 2 \mathrm{~A}-\mathrm{C} 22 \mathrm{~A}-\mathrm{H} 22 \mathrm{~A}$ & 125.5 \\
\hline $\mathrm{C} 21 \mathrm{~A}-\mathrm{C} 22 \mathrm{~A}-\mathrm{Fe} 2 \mathrm{~A}$ & $70.6(2)$ \\
\hline $\mathrm{C} 21 \mathrm{~A}-\mathrm{C} 22 \mathrm{~A}-\mathrm{H} 22 \mathrm{~A}$ & 125.7 \\
\hline $\mathrm{C} 21 \mathrm{~A}-\mathrm{C} 22 \mathrm{~A}-\mathrm{C} 23 \mathrm{~A}$ & $108.7(4)$ \\
\hline $\mathrm{C} 23 \mathrm{~A}-\mathrm{C} 22 \mathrm{~A}-\mathrm{Fe} 2 \mathrm{~A}$ & $69.9(2)$ \\
\hline $\mathrm{C} 23 \mathrm{~A}-\mathrm{C} 22 \mathrm{~A}-\mathrm{H} 22 \mathrm{~A}$ & 125.7 \\
\hline $\mathrm{Fe} 2 \mathrm{~A}-\mathrm{C} 23 \mathrm{~A}-\mathrm{H} 23 \mathrm{~A}$ & 125.8 \\
\hline $\mathrm{C} 22 \mathrm{~A}-\mathrm{C} 23 \mathrm{~A}-\mathrm{Fe} 2 \mathrm{~A}$ & $69.46(19)$ \\
\hline $\mathrm{C} 22 \mathrm{~A}-\mathrm{C} 23 \mathrm{~A}-\mathrm{H} 23 \mathrm{~A}$ & 126.5 \\
\hline $\mathrm{C} 22 \mathrm{~A}-\mathrm{C} 23 \mathrm{~A}-\mathrm{C} 24 \mathrm{~A}$ & $107.0(3)$ \\
\hline $\mathrm{C} 24 \mathrm{~A}-\mathrm{C} 23 \mathrm{~A}-\mathrm{Fe} 2 \mathrm{~A}$ & $69.81(19)$ \\
\hline $\mathrm{C} 24 \mathrm{~A}-\mathrm{C} 23 \mathrm{~A}-\mathrm{H} 23 \mathrm{~A}$ & 126.5 \\
\hline $\mathrm{Fe} 2 \mathrm{~A}-\mathrm{C} 24 \mathrm{~A}-\mathrm{H} 24 \mathrm{~A}$ & 125.9 \\
\hline $\mathrm{C} 20 \mathrm{~A}-\mathrm{C} 24 \mathrm{~A}-\mathrm{Fe} 2 \mathrm{~A}$ & $70.4(2)$ \\
\hline $\mathrm{C} 20 \mathrm{~A}-\mathrm{C} 24 \mathrm{~A}-\mathrm{C} 23 \mathrm{~A}$ & $108.5(4)$ \\
\hline $\mathrm{C} 20 \mathrm{~A}-\mathrm{C} 24 \mathrm{~A}-\mathrm{H} 24 \mathrm{~A}$ & 125.8 \\
\hline $\mathrm{C} 23 \mathrm{~A}-\mathrm{C} 24 \mathrm{~A}-\mathrm{Fe} 2 \mathrm{~A}$ & $69.5(2)$ \\
\hline $\mathrm{C} 23 \mathrm{~A}-\mathrm{C} 24 \mathrm{~A}-\mathrm{H} 24 \mathrm{~A}$ & 125.8 \\
\hline $\mathrm{C} 16 \mathrm{~A}-\mathrm{C} 25 \mathrm{~A}-\mathrm{H} 25 \mathrm{~A}$ & 117.4 \\
\hline $\mathrm{C} 26 \mathrm{~A}-\mathrm{C} 25 \mathrm{~A}-\mathrm{C} 16 \mathrm{~A}$ & $125.2(3)$ \\
\hline $\mathrm{C} 26 \mathrm{~A}-\mathrm{C} 25 \mathrm{~A}-\mathrm{H} 25 \mathrm{~A}$ & 117.4 \\
\hline $\mathrm{C} 25 \mathrm{~A}-\mathrm{C} 26 \mathrm{~A}-\mathrm{H} 26 \mathrm{~A}$ & 120.0 \\
\hline $\mathrm{C} 25 \mathrm{~A}-\mathrm{C} 26 \mathrm{~A}-\mathrm{H} 26 \mathrm{~B}$ & 120.0 \\
\hline & \\
\hline
\end{tabular}

\begin{tabular}{|c|c|}
\hline $\mathrm{Fe} 2 \mathrm{~B}-\mathrm{C} 18 \mathrm{~B}-\mathrm{H} 18 \mathrm{~B}$ & 126.2 \\
\hline $\mathrm{C} 17 \mathrm{~B}-\mathrm{C} 18 \mathrm{~B}-\mathrm{Fe} 2 \mathrm{~B}$ & $70.00(18)$ \\
\hline $\mathrm{C} 17 \mathrm{~B}-\mathrm{C} 18 \mathrm{~B}-\mathrm{H} 18 \mathrm{~B}$ & 125.5 \\
\hline $\mathrm{C} 17 \mathrm{~B}-\mathrm{C} 18 \mathrm{~B}-\mathrm{C} 19 \mathrm{~B}$ & $109.0(3)$ \\
\hline $\mathrm{C} 19 \mathrm{~B}-\mathrm{C} 18 \mathrm{~B}-\mathrm{Fe} 2 \mathrm{~B}$ & $69.88(17)$ \\
\hline $\mathrm{C} 19 \mathrm{~B}-\mathrm{C} 18 \mathrm{~B}-\mathrm{H} 18 \mathrm{~B}$ & 125.5 \\
\hline $\mathrm{Fe} 2 \mathrm{~B}-\mathrm{C} 19 \mathrm{~B}-\mathrm{H} 19 \mathrm{~B}$ & 127.0 \\
\hline $\mathrm{C} 15 \mathrm{~B}-\mathrm{C} 19 \mathrm{~B}-\mathrm{Fe} 2 \mathrm{~B}$ & $69.13(17)$ \\
\hline $\mathrm{C} 15 \mathrm{~B}-\mathrm{C} 19 \mathrm{~B}-\mathrm{H} 19 \mathrm{~B}$ & 125.9 \\
\hline $\mathrm{C} 18 \mathrm{~B}-\mathrm{C} 19 \mathrm{~B}-\mathrm{Fe} 2 \mathrm{~B}$ & $69.62(18)$ \\
\hline $\mathrm{C} 18 \mathrm{~B}-\mathrm{C} 19 \mathrm{~B}-\mathrm{C} 15 \mathrm{~B}$ & $108.3(3)$ \\
\hline $\mathrm{C} 18 \mathrm{~B}-\mathrm{C} 19 \mathrm{~B}-\mathrm{H} 19 \mathrm{~B}$ & 125.9 \\
\hline $\mathrm{Fe} 2 \mathrm{~B}-\mathrm{C} 20 \mathrm{~B}-\mathrm{H} 20 \mathrm{~B}$ & 126.3 \\
\hline $\mathrm{C} 21 \mathrm{~B}-\mathrm{C} 20 \mathrm{~B}-\mathrm{Fe} 2 \mathrm{~B}$ & 69.29 (19) \\
\hline $\mathrm{C} 21 \mathrm{~B}-\mathrm{C} 20 \mathrm{~B}-\mathrm{H} 20 \mathrm{~B}$ & 126.0 \\
\hline $\mathrm{C} 24 \mathrm{~B}-\mathrm{C} 20 \mathrm{~B}-\mathrm{Fe} 2 \mathrm{~B}$ & $69.98(19)$ \\
\hline $\mathrm{C} 24 \mathrm{~B}-\mathrm{C} 20 \mathrm{~B}-\mathrm{H} 20 \mathrm{~B}$ & 126.0 \\
\hline $\mathrm{C} 24 \mathrm{~B}-\mathrm{C} 20 \mathrm{~B}-\mathrm{C} 21 \mathrm{~B}$ & $107.9(3)$ \\
\hline $\mathrm{Fe} 2 \mathrm{~B}-\mathrm{C} 21 \mathrm{~B}-\mathrm{H} 21 \mathrm{~B}$ & 126.2 \\
\hline $\mathrm{C} 20 \mathrm{~B}-\mathrm{C} 21 \mathrm{~B}-\mathrm{Fe} 2 \mathrm{~B}$ & $70.0(2)$ \\
\hline $\mathrm{C} 20 \mathrm{~B}-\mathrm{C} 21 \mathrm{~B}-\mathrm{H} 21 \mathrm{~B}$ & 125.8 \\
\hline $\mathrm{C} 20 \mathrm{~B}-\mathrm{C} 21 \mathrm{~B}-\mathrm{C} 22 \mathrm{~B}$ & $108.4(4)$ \\
\hline $\mathrm{C} 22 \mathrm{~B}-\mathrm{C} 21 \mathrm{~B}-\mathrm{Fe} 2 \mathrm{~B}$ & $69.6(2)$ \\
\hline $\mathrm{C} 22 \mathrm{~B}-\mathrm{C} 21 \mathrm{~B}-\mathrm{H} 21 \mathrm{~B}$ & 125.8 \\
\hline $\mathrm{Fe} 2 \mathrm{~B}-\mathrm{C} 22 \mathrm{~B}-\mathrm{H} 22 \mathrm{~B}$ & 125.8 \\
\hline $\mathrm{C} 21 \mathrm{~B}-\mathrm{C} 22 \mathrm{~B}-\mathrm{Fe} 2 \mathrm{~B}$ & $69.55(19)$ \\
\hline $\mathrm{C} 21 \mathrm{~B}-\mathrm{C} 22 \mathrm{~B}-\mathrm{H} 22 \mathrm{~B}$ & 126.4 \\
\hline $\mathrm{C} 23 \mathrm{~B}-\mathrm{C} 22 \mathrm{~B}-\mathrm{Fe} 2 \mathrm{~B}$ & $69.8(2)$ \\
\hline $\mathrm{C} 23 \mathrm{~B}-\mathrm{C} 22 \mathrm{~B}-\mathrm{C} 21 \mathrm{~B}$ & $107.2(4)$ \\
\hline $\mathrm{C} 23 \mathrm{~B}-\mathrm{C} 22 \mathrm{~B}-\mathrm{H} 22 \mathrm{~B}$ & 126.4 \\
\hline $\mathrm{Fe} 2 \mathrm{~B}-\mathrm{C} 23 \mathrm{~B}-\mathrm{H} 23 \mathrm{~B}$ & 126.2 \\
\hline $\mathrm{C} 22 \mathrm{~B}-\mathrm{C} 23 \mathrm{~B}-\mathrm{Fe} 2 \mathrm{~B}$ & $69.8(2)$ \\
\hline $\mathrm{C} 22 \mathrm{~B}-\mathrm{C} 23 \mathrm{~B}-\mathrm{H} 23 \mathrm{~B}$ & 125.6 \\
\hline $\mathrm{C} 22 \mathrm{~B}-\mathrm{C} 23 \mathrm{~B}-\mathrm{C} 24 \mathrm{~B}$ & $108.7(3)$ \\
\hline $\mathrm{C} 24 \mathrm{~B}-\mathrm{C} 23 \mathrm{~B}-\mathrm{Fe} 2 \mathrm{~B}$ & $69.96(19)$ \\
\hline $\mathrm{C} 24 \mathrm{~B}-\mathrm{C} 23 \mathrm{~B}-\mathrm{H} 23 \mathrm{~B}$ & 125.6 \\
\hline $\mathrm{Fe} 2 \mathrm{~B}-\mathrm{C} 24 \mathrm{~B}-\mathrm{H} 24 \mathrm{~B}$ & 126.4 \\
\hline $\mathrm{C} 20 \mathrm{~B}-\mathrm{C} 24 \mathrm{~B}-\mathrm{Fe} 2 \mathrm{~B}$ & 69.89 (19) \\
\hline $\mathrm{C} 20 \mathrm{~B}-\mathrm{C} 24 \mathrm{~B}-\mathrm{C} 23 \mathrm{~B}$ & $107.8(4)$ \\
\hline $\mathrm{C} 20 \mathrm{~B}-\mathrm{C} 24 \mathrm{~B}-\mathrm{H} 24 \mathrm{~B}$ & 126.1 \\
\hline $\mathrm{C} 23 \mathrm{~B}-\mathrm{C} 24 \mathrm{~B}-\mathrm{Fe} 2 \mathrm{~B}$ & $69.2(2)$ \\
\hline $\mathrm{C} 23 \mathrm{~B}-\mathrm{C} 24 \mathrm{~B}-\mathrm{H} 24 \mathrm{~B}$ & 126.1 \\
\hline $\mathrm{C} 16 \mathrm{~B}-\mathrm{C} 25 \mathrm{~B}-\mathrm{H} 25 \mathrm{~B}$ & 117.5 \\
\hline $\mathrm{C} 26 \mathrm{~B}-\mathrm{C} 25 \mathrm{~B}-\mathrm{C} 16 \mathrm{~B}$ & $125.1(4)$ \\
\hline $\mathrm{C} 26 \mathrm{~B}-\mathrm{C} 25 \mathrm{~B}-\mathrm{H} 25 \mathrm{~B}$ & 117.5 \\
\hline $\mathrm{C} 25 \mathrm{~B}-\mathrm{C} 26 \mathrm{~B}-\mathrm{H} 26 \mathrm{C}$ & 120.0 \\
\hline $\mathrm{C} 25 \mathrm{~B}-\mathrm{C} 26 \mathrm{~B}-\mathrm{H} 26 \mathrm{D}$ & 120.0 \\
\hline $\mathrm{H} 26 \mathrm{C}-\mathrm{C} 26 \mathrm{~B}-\mathrm{H} 26 \mathrm{D}$ & 120.0 \\
\hline
\end{tabular}




$\begin{array}{ll}\text { C35A-C34A-P1A } & 119.2(2) \\ \text { C35A-C34A-C39A } & 119.7(3) \\ \text { C39A-C34A-P1A } & 121.1(2) \\ \text { C34A-C35A-H35A } & 120.1 \\ \text { C36A-C35A-C34A } & 119.9(3) \\ \text { C36A-C35A-H35A } & 120.1 \\ \text { C35A-C36A-H36A } & 119.9 \\ \text { C37A-C36A-C35A } & 120.3(3) \\ \text { C37A-C36A-H36A } & 119.9 \\ \text { C36A-C37A-H37A } & 119.9 \\ \text { C38A-C37A-C36A } & 120.1(3) \\ \text { C38A-C37A-H37A } & 119.9 \\ \text { C37A-C38A-H38A } & 119.8 \\ \text { C37A-C38A-C39A } & 120.4(3) \\ \text { C39A-C38A-H38A } & 119.8 \\ \text { C34A-C39A-H39A } & 120.2 \\ \text { C38A-C39A-C34A } & 119.6(3) \\ \text { C38A-C39A-H39A } & 120.2 \\ & \\ \text { Fe1A-C1A-C2A-C3A } & 58.8(2) \\ \text { Fe1A-C1A-C2A-C11A } & -119.1(3) \\ \text { Fe1A-C1A-C5A-C4A } & -59.6(2) \\ \text { Fe1A-C2A-C3A-C4A } & 59.6(2) \\ \text { Fe1A-C2A-C11A-C12A } & 99.8(4) \\ \text { Fe1A-C3A-C4A-C5A } & 57.9(2) \\ \text { Fe1A-C4A-C5A-C1A } & 58.2(2) \\ \text { Fe1A-C6A-C7A-C8A } & -59.7(2) \\ \text { Fe1A-C6A-C10A-C9A } & 59.2(2) \\ \text { Fe1A-C7A-C8A-C9A } & -59.8(3) \\ \text { Fe1A-C8A-C9A-C10A } & -59.4(3) \\ \text { Fe1A-C9A-C10A-C6A } & -58.9(2) \\ \text { Fe2A-C15A-C16A-C17A } & 59.0(2) \\ \text { Fe2A-C15A-C16A-C25A } & -120.5(3) \\ \text { Fe2A-C15A-C19A-C18A } & -59.0(2) \\ \text { Fe2A-C16A-C17A-C18A } & 59.4(2) \\ \text { Fe2A-C16A-C25A-C26A } & 111.9(4) \\ \text { Fe2A-C17A-C18A-C19A } & 58.9(2) \\ \text { Fe2A-C18A-C19A-C15A } & 58.1(2) \\ \text { Fe2A-C20A-C21A-C22A } & -59.1(2) \\ \text { Fe2A-C20A-C24A-C23A } & 59.3(2) \\ \text { Fe2A-C21A-C22A-C23A } & -59.7(3) \\ \text { Fe2A-C22A-C23A-C24A } & -60.0(2) \\ \text { Fe2A-C23A-C24A-C20A } & -59.8(2) \\ \text { S1A-P1A-C1A-Fe1A } & 56.0(2) \\ \text { S1A-P1A-C1A-C2A } & -41.1(3) \\ \text { S1A-P1A-C1A-C5A } & 146.9(2) \\ \text { S1A-P1A-C15A-Fe2A } & 44.7(2) \\ \text { S1A-P1A-C15A-C16A } & -52.8(3) \\ & \end{array}$

$\begin{array}{ll}\mathrm{C} 35 \mathrm{~B}-\mathrm{C} 34 \mathrm{~B}-\mathrm{P} 1 \mathrm{~B} & 121.1(2) \\ \mathrm{C} 39 \mathrm{~B}-\mathrm{C} 34 \mathrm{~B}-\mathrm{P} 1 \mathrm{~B} & 119.7(2) \\ \mathrm{C} 39 \mathrm{~B}-\mathrm{C} 34 \mathrm{~B}-\mathrm{C} 35 \mathrm{~B} & 119.2(3) \\ \mathrm{C} 34 \mathrm{~B}-\mathrm{C} 35 \mathrm{~B}-\mathrm{H} 35 \mathrm{~B} & 119.9 \\ \mathrm{C} 36 \mathrm{~B}-\mathrm{C} 35 \mathrm{~B}-\mathrm{C} 34 \mathrm{~B} & 120.3(3) \\ \mathrm{C} 36 \mathrm{~B}-\mathrm{C} 35 \mathrm{~B}-\mathrm{H} 35 \mathrm{~B} & 119.9 \\ \mathrm{C} 35 \mathrm{~B}-\mathrm{C} 36 \mathrm{~B}-\mathrm{H} 36 \mathrm{~B} & 120.2 \\ \mathrm{C} 37 \mathrm{~B}-\mathrm{C} 36 \mathrm{~B}-\mathrm{C} 35 \mathrm{~B} & 119.5(3) \\ \mathrm{C} 37 \mathrm{~B}-\mathrm{C} 36 \mathrm{~B}-\mathrm{H} 36 \mathrm{~B} & 120.2 \\ \mathrm{C} 36 \mathrm{~B}-\mathrm{C} 37 \mathrm{~B}-\mathrm{H} 37 \mathrm{~B} & 119.5 \\ \mathrm{C} 38 \mathrm{~B}-\mathrm{C} 37 \mathrm{~B}-\mathrm{C} 36 \mathrm{~B} & 120.9(3) \\ \mathrm{C} 38 \mathrm{~B}-\mathrm{C} 37 \mathrm{~B}-\mathrm{H} 37 \mathrm{~B} & 119.5 \\ \mathrm{C} 37 \mathrm{~B}-\mathrm{C} 38 \mathrm{~B}-\mathrm{H} 38 \mathrm{~B} & 120.2 \\ \mathrm{C} 37 \mathrm{~B}-\mathrm{C} 38 \mathrm{~B}-\mathrm{C} 39 \mathrm{~B} & 119.6(3) \\ \mathrm{C} 39 \mathrm{~B}-\mathrm{C} 38 \mathrm{~B}-\mathrm{H} 38 \mathrm{~B} & 120.2 \\ \mathrm{C} 34 \mathrm{~B}-\mathrm{C} 39 \mathrm{~B}-\mathrm{C} 38 \mathrm{~B} & 120.5(3) \\ \text { C34B-C39B-H39B } & 119.7 \\ \mathrm{C} 38 \mathrm{~B}-\mathrm{C} 39 \mathrm{~B}-\mathrm{H} 39 \mathrm{~B} & 119.7\end{array}$

$\mathrm{Fe} 1 \mathrm{~B}-\mathrm{C} 1 \mathrm{~B}-\mathrm{C} 2 \mathrm{~B}-\mathrm{C} 3 \mathrm{~B} \quad 59.2(2)$

$\mathrm{Fe} 1 \mathrm{~B}-\mathrm{C} 1 \mathrm{~B}-\mathrm{C} 2 \mathrm{~B}-\mathrm{C} 11 \mathrm{~B} \quad-118.1$ (3)

$\mathrm{Fe} 1 \mathrm{~B}-\mathrm{C} 1 \mathrm{~B}-\mathrm{C} 5 \mathrm{~B}-\mathrm{C} 4 \mathrm{~B} \quad-59.8(2)$

$\mathrm{Fe} 1 \mathrm{~B}-\mathrm{C} 2 \mathrm{~B}-\mathrm{C} 3 \mathrm{~B}-\mathrm{C} 4 \mathrm{~B} \quad 59.7$ (2)

$\mathrm{Fe} 1 \mathrm{~B}-\mathrm{C} 2 \mathrm{~B}-\mathrm{C} 11 \mathrm{~B}-\mathrm{C} 12 \mathrm{~B} \quad 108.8(4)$

$\mathrm{Fe} 1 \mathrm{~B}-\mathrm{C} 3 \mathrm{~B}-\mathrm{C} 4 \mathrm{~B}-\mathrm{C} 5 \mathrm{~B} \quad 57.6(2)$

$\mathrm{Fe} 1 \mathrm{~B}-\mathrm{C} 4 \mathrm{~B}-\mathrm{C} 5 \mathrm{~B}-\mathrm{C} 1 \mathrm{~B} \quad 58.5$ (2)

$\mathrm{Fe} 1 \mathrm{~B}-\mathrm{C} 6 \mathrm{~B}-\mathrm{C} 7 \mathrm{~B}-\mathrm{C} 8 \mathrm{~B} \quad-59.5$ (3)

$\mathrm{Fe} 1 \mathrm{~B}-\mathrm{C} 6 \mathrm{~B}-\mathrm{C} 10 \mathrm{~B}-\mathrm{C} 9 \mathrm{~B} \quad 58.9$ (3)

$\mathrm{Fe} 1 \mathrm{~B}-\mathrm{C} 7 \mathrm{~B}-\mathrm{C} 8 \mathrm{~B}-\mathrm{C} 9 \mathrm{~B} \quad-60.1$ (3)

$\mathrm{Fe} 1 \mathrm{~B}-\mathrm{C} 8 \mathrm{~B}-\mathrm{C} 9 \mathrm{~B}-\mathrm{C} 10 \mathrm{~B} \quad-60.3$ (3)

$\mathrm{Fe} 1 \mathrm{~B}-\mathrm{C} 9 \mathrm{~B}-\mathrm{C} 10 \mathrm{~B}-\mathrm{C} 6 \mathrm{~B} \quad-59.0$ (2)

$\mathrm{Fe} 2 \mathrm{~B}-\mathrm{C} 15 \mathrm{~B}-\mathrm{C} 16 \mathrm{~B}-\mathrm{C} 17 \mathrm{~B} \quad 59.1(2)$

$\mathrm{Fe} 2 \mathrm{~B}-\mathrm{C} 15 \mathrm{~B}-\mathrm{C} 16 \mathrm{~B}-\mathrm{C} 25 \mathrm{~B} \quad-120.1$ (3)

$\mathrm{Fe} 2 \mathrm{~B}-\mathrm{C} 15 \mathrm{~B}-\mathrm{C} 19 \mathrm{~B}-\mathrm{C} 18 \mathrm{~B} \quad-58.8$ (2)

$\mathrm{Fe} 2 \mathrm{~B}-\mathrm{C} 16 \mathrm{~B}-\mathrm{C} 17 \mathrm{~B}-\mathrm{C} 18 \mathrm{~B} \quad 58.9(2)$

$\mathrm{Fe} 2 \mathrm{~B}-\mathrm{C} 16 \mathrm{~B}-\mathrm{C} 25 \mathrm{~B}-\mathrm{C} 26 \mathrm{~B} \quad 100.9$ (5)

$\mathrm{Fe} 2 \mathrm{~B}-\mathrm{C} 17 \mathrm{~B}-\mathrm{C} 18 \mathrm{~B}-\mathrm{C} 19 \mathrm{~B} \quad 59.2(2)$

$\mathrm{Fe} 2 \mathrm{~B}-\mathrm{C} 18 \mathrm{~B}-\mathrm{C} 19 \mathrm{~B}-\mathrm{C} 15 \mathrm{~B} \quad 58.5$ (2)

$\mathrm{Fe} 2 \mathrm{~B}-\mathrm{C} 20 \mathrm{~B}-\mathrm{C} 21 \mathrm{~B}-\mathrm{C} 22 \mathrm{~B} \quad-59.2(2)$

$\mathrm{Fe} 2 \mathrm{~B}-\mathrm{C} 20 \mathrm{~B}-\mathrm{C} 24 \mathrm{~B}-\mathrm{C} 23 \mathrm{~B} \quad 59.0$ (2)

$\mathrm{Fe} 2 \mathrm{~B}-\mathrm{C} 21 \mathrm{~B}-\mathrm{C} 22 \mathrm{~B}-\mathrm{C} 23 \mathrm{~B}-59.9$ (3)

$\mathrm{Fe} 2 \mathrm{~B}-\mathrm{C} 22 \mathrm{~B}-\mathrm{C} 23 \mathrm{~B}-\mathrm{C} 24 \mathrm{~B}-59.3$ (3)

$\mathrm{Fe} 2 \mathrm{~B}-\mathrm{C} 23 \mathrm{~B}-\mathrm{C} 24 \mathrm{~B}-\mathrm{C} 20 \mathrm{~B} \quad-59.5$ (2)

$\mathrm{S} 1 \mathrm{~B}-\mathrm{P} 1 \mathrm{~B}-\mathrm{C} 1 \mathrm{~B}-\mathrm{Fe} 1 \mathrm{~B} \quad 58.5$ (2)

$\mathrm{S} 1 \mathrm{~B}-\mathrm{P} 1 \mathrm{~B}-\mathrm{C} 1 \mathrm{~B}-\mathrm{C} 2 \mathrm{~B} \quad-39.0(3)$

$\mathrm{S} 1 \mathrm{~B}-\mathrm{P} 1 \mathrm{~B}-\mathrm{C} 1 \mathrm{~B}-\mathrm{C} 5 \mathrm{~B} \quad 149.5$ (2)

$\mathrm{S} 1 \mathrm{~B}-\mathrm{P} 1 \mathrm{~B}-\mathrm{C} 15 \mathrm{~B}-\mathrm{Fe} 2 \mathrm{~B} \quad 45.0$ (2)

$\mathrm{S} 1 \mathrm{~B}-\mathrm{P} 1 \mathrm{~B}-\mathrm{C} 15 \mathrm{~B}-\mathrm{C} 16 \mathrm{~B} \quad-49.2$ (3) 


$\begin{array}{ll}\text { S1A-P1A-C15A-C19A } & 138.5(2) \\ \text { S1A-P1A-C34A-C35A } & -32.4(3) \\ \text { S1A-P1A-C34A-C39A } & 149.3(2) \\ \text { P1A-C1A-C2A-Fe1A } & 126.5(2) \\ \text { P1A-C1A-C2A-C3A } & -174.8(2) \\ \text { P1A-C1A-C2A-C11A } & 7.4(5) \\ \text { P1A-C1A-C5A-Fe1A } & -125.8(2) \\ \text { P1A-C1A-C5A-C4A } & 174.6(2) \\ \text { P1A-C15A-C16A-Fe2A } & 129.6(2) \\ \text { P1A-C15A-C16A-C17A } & -171.3(2) \\ \text { P1A-C15A-C16A-C25A } & 9.1(5) \\ \text { P1A-C15A-C19A-Fe2A } & -129.3(2) \\ \text { P1A-C15A-C19A-C18A } & 171.7(2) \\ \text { P1A-C34A-C35A-C36A } & -176.4(2) \\ \text { P1A-C34A-C39A-C38A } & 177.1(2) \\ \text { C1A-P1A-C15A-Fe2A } & -84.1(2) \\ \text { C1A-P1A-C15A-C16A } & 178.3(3) \\ \text { C1A-P1A-C15A-C19A } & 9.7(3) \\ \text { C1A-P1A-C34A-C35A } & 93.5(2) \\ \text { C1A-P1A-C34A-C39A } & -84.8(3) \\ \text { C1A-C2A-C3A-Fe1A } & -57.69(19) \\ \text { C1A-C2A-C3A-C4A } & 1.9(3) \\ \text { C1A-C2A-C11A-C12A } & -172.1(4) \\ \text { C2A-C1A-C5A-Fe1A } & 60.7(2) \\ \text { C2A-C1A-C5A-C4A } & 1.1(3) \\ \text { C2A-C3A-C4A-Fe1A } & -59.1(2) \\ \text { C2A-C3A-C4A-C5A } & -1.2(4) \\ \text { C3A-C2A-C11A-C12A } & 10.5(6) \\ \text { C3A-C4A-C5A-Fe1A } & -58.1(2) \\ \text { C3A-C4A-C5A-C1A } & 0.0(4) \\ \text { C5A-C1A-C2A-Fe1A } & -60.6(2) \\ \text { C5A-C1A-C2A-C3A } & -1.9(3) \\ \text { C5A-C1A-C2A-C11A } & -179.7(3) \\ \text { C6A-C7A-C8A-Fe1A } & 59.8(2) \\ \text { C6A-C7A-C8A-C9A } & 0.0(4) \\ \text { C7A-C6A-C10A-Fe1A } & -59.4(2) \\ \text { C7A-C6A-C10A-C9A } & -0.2(4) \\ \text { C7A-C8A-C9A-Fe1A } & 59.3(2) \\ \text { C7A-C8A-C9A-C10A } & -0.1(4) \\ \text { C8A-C9A-C10A-Fe1A } & 59.1(3) \\ \text { C8A-C9A-C10A-C6A } & 0.2(4) \\ \text { C10A-C6A-C7A-Fe1A } & 59.9(2) \\ \text { C10A-C6A-C7A-C8A } & 0.2(4) \\ \text { C11A-C2A-C3A-Fe1A } & 120.2(3) \\ \text { C11A-C2A-C3A-C4A } & 179.8(3) \\ \text { C15A-P1A-C1A-Fe1A } & -174.24(19) \\ \text { C15A-P1A-C1A-C2A } & 88.6(3) \\ \text { C15A-P1A-C1A-C5A } & -83.4(3) \\ & \end{array}$

$\begin{array}{ll}\text { S1B-P1B-C15B-C19B } & 135.5(2) \\ \text { S1B-P1B-C34B-C35B } & 150.6(2) \\ \text { S1B-P1B-C34B-C39B } & -32.2(3) \\ \text { P1B-C1B-C2B-Fe1B } & 127.6(2) \\ \text { P1B-C1B-C2B-C3B } & -173.3(2) \\ \text { P1B-C1B-C2B-C11B } & 9.5(5) \\ \text { P1B-C1B-C5B-Fe1B } & -127.0(2) \\ \text { P1B-C1B-C5B-C4B } & 173.2(2) \\ \text { P1B-C15B-C16B-Fe2B } & 124.4(2) \\ \text { P1B-C15B-C16B-C17B } & -176.5(2) \\ \text { P1B-C15B-C16B-C25B } & 4.3(5) \\ \text { P1B-C15B-C19B-Fe2B } & -124.2(2) \\ \text { P1B-C15B-C19B-C18B } & 177.0(2) \\ \text { P1B-C34B-C35B-C36B } & 175.4(2) \\ \text { P1B-C34B-C39B-C38B } & -175.7(2) \\ \text { C1B-P1B-C15B-Fe2B } & -83.3(2) \\ \text { C1B-P1B-C15B-C16B } & -177.6(3) \\ \text { C1B-P1B-C15B-C19B } & 7.1(3) \\ \text { C1B-P1B-C34B-C35B } & -83.7(3) \\ \text { C1B-P1B-C34B-C39B } & 93.4(2) \\ \text { C1B-C2B-C3B-Fe1B } & -58.46(19) \\ \text { C1B-C2B-C3B-C4B } & 1.2(3) \\ \text { C1B-C2B-C11B-C12B } & -162.7(3) \\ \text { C2B-C1B-C5B-Fe1B } & 59.9(2) \\ \text { C2B-C1B-C5B-C4B } & 0.0(3) \\ \text { C2B-C3B-C4B-Fe1B } & -58.8(2) \\ \text { C2B-C3B-C4B-C5B } & -1.2(4) \\ \text { C3B-C2B-C11B-C12B } & 20.5(5) \\ \text { C3B-C4B-C5B-Fe1B } & -57.8(2) \\ \text { C3B-C4B-C5B-C1B } & 0.7(4) \\ \text { C5B-C1B-C2B-Fe1B } & -59.9(2) \\ \text { C5B-C1B-C2B-C3B } & -0.8(3) \\ \text { C5B-C1B-C2B-C11B } & -178.0(3) \\ \text { C6B-C7B-C8B-Fe1B } & 60.5(2) \\ \text { C6B-C7B-C8B-C9B } & 0.3(4) \\ \text { C7B-C6B-C10B-Fe1B } & -59.1(3) \\ \text { C7B-C6B-C10B-C9B } & -0.1(4) \\ \text { C7B-C8B-C9B-Fe1B } & 59.9(3) \\ \text { C7B-C8B-C9B-C10B } & -0.4(4) \\ \text { C8B-C9B-C10B-Fe1B } & 59.3(3) \\ \text { C8B-C9B-C10B-C6B } & 0.4(4) \\ \text { C10B-C6B-C7B-Fe1B } & 59.3(3) \\ \text { C10B-C6B-C7B-C8B } & -0.1(4) \\ \text { C11B-C2B-C3B-Fe1B } & 118.9(3) \\ \text { C11B-C2B-C3B-C4B } & 178.6(3) \\ \text { C15B-P1B-C1B-Fe1B } & -172.69(19) \\ \text { C15B-P1B-C1B-C2B } & 89.8(3) \\ \text { C15B-P1B-C1B-C5B } & -81.7(3) \\ & \end{array}$




\begin{tabular}{|c|c|c|c|}
\hline $\mathrm{C} 15 \mathrm{~A}-\mathrm{P} 1 \mathrm{~A}-\mathrm{C} 34 \mathrm{~A}-\mathrm{C} 35 \mathrm{~A}$ & $-159.7(2)$ & $\mathrm{C} 15 \mathrm{~B}-\mathrm{P} 1 \mathrm{~B}-\mathrm{C} 34 \mathrm{~B}-\mathrm{C} 35 \mathrm{~B}$ & $23.5(3)$ \\
\hline $\mathrm{C} 15 \mathrm{~A}-\mathrm{P} 1 \mathrm{~A}-\mathrm{C} 34 \mathrm{~A}-\mathrm{C} 39 \mathrm{~A}$ & $22.0(3)$ & $\mathrm{C} 15 \mathrm{~B}-\mathrm{P} 1 \mathrm{~B}-\mathrm{C} 34 \mathrm{~B}-\mathrm{C} 39 \mathrm{~B}$ & $-159.4(2)$ \\
\hline $\mathrm{C} 15 \mathrm{~A}-\mathrm{C} 16 \mathrm{~A}-\mathrm{C} 17 \mathrm{~A}-\mathrm{Fe} 2 \mathrm{~A}$ & $-58.5(2)$ & $\mathrm{C} 15 \mathrm{~B}-\mathrm{C} 16 \mathrm{~B}-\mathrm{C} 17 \mathrm{~B}-\mathrm{Fe} 2 \mathrm{~B}$ & $-58.7(2)$ \\
\hline $\mathrm{C} 15 \mathrm{~A}-\mathrm{C} 16 \mathrm{~A}-\mathrm{C} 17 \mathrm{~A}-\mathrm{C} 18 \mathrm{~A}$ & $0.9(3)$ & $\mathrm{C} 15 \mathrm{~B}-\mathrm{C} 16 \mathrm{~B}-\mathrm{C} 17 \mathrm{~B}-\mathrm{C} 18 \mathrm{~B}$ & $0.1(4)$ \\
\hline $\mathrm{C} 15 \mathrm{~A}-\mathrm{C} 16 \mathrm{~A}-\mathrm{C} 25 \mathrm{~A}-\mathrm{C} 26 \mathrm{~A}$ & $-159.1(4)$ & $\mathrm{C} 15 \mathrm{~B}-\mathrm{C} 16 \mathrm{~B}-\mathrm{C} 25 \mathrm{~B}-\mathrm{C} 26 \mathrm{~B}$ & $-170.2(4)$ \\
\hline $\mathrm{C} 16 \mathrm{~A}-\mathrm{C} 15 \mathrm{~A}-\mathrm{C} 19 \mathrm{~A}-\mathrm{Fe} 2 \mathrm{~A}$ & $60.06(19)$ & $\mathrm{C} 16 \mathrm{~B}-\mathrm{C} 15 \mathrm{~B}-\mathrm{C} 19 \mathrm{~B}-\mathrm{Fe} 2 \mathrm{~B}$ & $59.6(2)$ \\
\hline $\mathrm{C} 16 \mathrm{~A}-\mathrm{C} 15 \mathrm{~A}-\mathrm{C} 19 \mathrm{~A}-\mathrm{C} 18 \mathrm{~A}$ & $1.0(3)$ & $\mathrm{C} 16 \mathrm{~B}-\mathrm{C} 15 \mathrm{~B}-\mathrm{C} 19 \mathrm{~B}-\mathrm{C} 18 \mathrm{~B}$ & $0.9(3)$ \\
\hline $\mathrm{C} 16 \mathrm{~A}-\mathrm{C} 17 \mathrm{~A}-\mathrm{C} 18 \mathrm{~A}-\mathrm{Fe} 2 \mathrm{~A}$ & $-59.1(2)$ & $\mathrm{C} 16 \mathrm{~B}-\mathrm{C} 17 \mathrm{~B}-\mathrm{C} 18 \mathrm{~B}-\mathrm{Fe} 2 \mathrm{~B}$ & $-58.8(2)$ \\
\hline $\mathrm{C} 16 \mathrm{~A}-\mathrm{C} 17 \mathrm{~A}-\mathrm{C} 18 \mathrm{~A}-\mathrm{C} 19 \mathrm{~A}$ & $-0.2(4)$ & $\mathrm{C} 16 \mathrm{~B}-\mathrm{C} 17 \mathrm{~B}-\mathrm{C} 18 \mathrm{~B}-\mathrm{C} 19 \mathrm{~B}$ & $0.4(4)$ \\
\hline $\mathrm{C} 17 \mathrm{~A}-\mathrm{C} 16 \mathrm{~A}-\mathrm{C} 25 \mathrm{~A}-\mathrm{C} 26 \mathrm{~A}$ & $21.5(6)$ & $\mathrm{C} 17 \mathrm{~B}-\mathrm{C} 16 \mathrm{~B}-\mathrm{C} 25 \mathrm{~B}-\mathrm{C} 26 \mathrm{~B}$ & $10.8(7)$ \\
\hline $\mathrm{C} 17 \mathrm{~A}-\mathrm{C} 18 \mathrm{~A}-\mathrm{C} 19 \mathrm{~A}-\mathrm{Fe} 2 \mathrm{~A}$ & $-58.6(2)$ & $\mathrm{C} 17 \mathrm{~B}-\mathrm{C} 18 \mathrm{~B}-\mathrm{C} 19 \mathrm{~B}-\mathrm{Fe} 2 \mathrm{~B}$ & $-59.3(2)$ \\
\hline $\mathrm{C} 17 \mathrm{~A}-\mathrm{C} 18 \mathrm{~A}-\mathrm{C} 19 \mathrm{~A}-\mathrm{C} 15 \mathrm{~A}$ & $-0.5(4)$ & $\mathrm{C} 17 \mathrm{~B}-\mathrm{C} 18 \mathrm{~B}-\mathrm{C} 19 \mathrm{~B}-\mathrm{C} 15 \mathrm{~B}$ & $-0.8(4)$ \\
\hline $\mathrm{C} 19 \mathrm{~A}-\mathrm{C} 15 \mathrm{~A}-\mathrm{C} 16 \mathrm{~A}-\mathrm{Fe} 2 \mathrm{~A}$ & $-60.23(19)$ & $\mathrm{C} 19 \mathrm{~B}-\mathrm{C} 15 \mathrm{~B}-\mathrm{C} 16 \mathrm{~B}-\mathrm{Fe} 2 \mathrm{~B}$ & $-59.7(2)$ \\
\hline $\mathrm{C} 19 \mathrm{~A}-\mathrm{C} 15 \mathrm{~A}-\mathrm{C} 16 \mathrm{~A}-\mathrm{C} 17 \mathrm{~A}$ & $-1.2(3)$ & $\mathrm{C} 19 \mathrm{~B}-\mathrm{C} 15 \mathrm{~B}-\mathrm{C} 16 \mathrm{~B}-\mathrm{C} 17 \mathrm{~B}$ & $-0.6(3)$ \\
\hline $\mathrm{C} 19 \mathrm{~A}-\mathrm{C} 15 \mathrm{~A}-\mathrm{C} 16 \mathrm{~A}-\mathrm{C} 25 \mathrm{~A}$ & $179.3(3)$ & $\mathrm{C} 19 \mathrm{~B}-\mathrm{C} 15 \mathrm{~B}-\mathrm{C} 16 \mathrm{~B}-\mathrm{C} 25 \mathrm{~B}$ & $-179.8(3)$ \\
\hline $\mathrm{C} 20 \mathrm{~A}-\mathrm{C} 21 \mathrm{~A}-\mathrm{C} 22 \mathrm{~A}-\mathrm{Fe} 2 \mathrm{~A}$ & $59.5(2)$ & $\mathrm{C} 20 \mathrm{~B}-\mathrm{C} 21 \mathrm{~B}-\mathrm{C} 22 \mathrm{~B}-\mathrm{Fe} 2 \mathrm{~B}$ & $59.4(2)$ \\
\hline $\mathrm{C} 20 \mathrm{~A}-\mathrm{C} 21 \mathrm{~A}-\mathrm{C} 22 \mathrm{~A}-\mathrm{C} 23 \mathrm{~A}$ & $-0.2(4)$ & $\mathrm{C} 20 \mathrm{~B}-\mathrm{C} 21 \mathrm{~B}-\mathrm{C} 22 \mathrm{~B}-\mathrm{C} 23 \mathrm{~B}$ & $-0.5(4)$ \\
\hline $\mathrm{C} 21 \mathrm{~A}-\mathrm{C} 20 \mathrm{~A}-\mathrm{C} 24 \mathrm{~A}-\mathrm{Fe} 2 \mathrm{~A}$ & $-59.4(2)$ & $\mathrm{C} 21 \mathrm{~B}-\mathrm{C} 20 \mathrm{~B}-\mathrm{C} 24 \mathrm{~B}-\mathrm{Fe} 2 \mathrm{~B}$ & $-59.1(2)$ \\
\hline $\mathrm{C} 21 \mathrm{~A}-\mathrm{C} 20 \mathrm{~A}-\mathrm{C} 24 \mathrm{~A}-\mathrm{C} 23 \mathrm{~A}$ & $-0.1(4)$ & $\mathrm{C} 21 \mathrm{~B}-\mathrm{C} 20 \mathrm{~B}-\mathrm{C} 24 \mathrm{~B}-\mathrm{C} 23 \mathrm{~B}$ & $-0.1(4)$ \\
\hline $\mathrm{C} 21 \mathrm{~A}-\mathrm{C} 22 \mathrm{~A}-\mathrm{C} 23 \mathrm{~A}-\mathrm{Fe} 2 \mathrm{~A}$ & $60.1(2)$ & $\mathrm{C} 21 \mathrm{~B}-\mathrm{C} 22 \mathrm{~B}-\mathrm{C} 23 \mathrm{~B}-\mathrm{Fe} 2 \mathrm{~B}$ & $59.8(2)$ \\
\hline $\mathrm{C} 21 \mathrm{~A}-\mathrm{C} 22 \mathrm{~A}-\mathrm{C} 23 \mathrm{~A}-\mathrm{C} 24 \mathrm{~A}$ & $0.2(4)$ & $\mathrm{C} 21 \mathrm{~B}-\mathrm{C} 22 \mathrm{~B}-\mathrm{C} 23 \mathrm{~B}-\mathrm{C} 24 \mathrm{~B}$ & $0.4(4)$ \\
\hline $\mathrm{C} 22 \mathrm{~A}-\mathrm{C} 23 \mathrm{~A}-\mathrm{C} 24 \mathrm{~A}-\mathrm{Fe} 2 \mathrm{~A}$ & $59.8(2)$ & $\mathrm{C} 22 \mathrm{~B}-\mathrm{C} 23 \mathrm{~B}-\mathrm{C} 24 \mathrm{~B}-\mathrm{Fe} 2 \mathrm{~B}$ & $59.3(3)$ \\
\hline $\mathrm{C} 22 \mathrm{~A}-\mathrm{C} 23 \mathrm{~A}-\mathrm{C} 24 \mathrm{~A}-\mathrm{C} 20 \mathrm{~A}$ & $-0.1(4)$ & $\mathrm{C} 22 \mathrm{~B}-\mathrm{C} 23 \mathrm{~B}-\mathrm{C} 24 \mathrm{~B}-\mathrm{C} 20 \mathrm{~B}$ & $-0.2(4)$ \\
\hline $\mathrm{C} 24 \mathrm{~A}-\mathrm{C} 20 \mathrm{~A}-\mathrm{C} 21 \mathrm{~A}-\mathrm{Fe} 2 \mathrm{~A}$ & $59.3(2)$ & $\mathrm{C} 24 \mathrm{~B}-\mathrm{C} 20 \mathrm{~B}-\mathrm{C} 21 \mathrm{~B}-\mathrm{Fe} 2 \mathrm{~B}$ & $59.5(2)$ \\
\hline $\mathrm{C} 24 \mathrm{~A}-\mathrm{C} 20 \mathrm{~A}-\mathrm{C} 21 \mathrm{~A}-\mathrm{C} 22 \mathrm{~A}$ & $0.2(4)$ & $\mathrm{C} 24 \mathrm{~B}-\mathrm{C} 20 \mathrm{~B}-\mathrm{C} 21 \mathrm{~B}-\mathrm{C} 22 \mathrm{~B}$ & $0.3(4)$ \\
\hline $\mathrm{C} 25 \mathrm{~A}-\mathrm{C} 16 \mathrm{~A}-\mathrm{C} 17 \mathrm{~A}-\mathrm{Fe} 2 \mathrm{~A}$ & $121.0(3)$ & $\mathrm{C} 25 \mathrm{~B}-\mathrm{C} 16 \mathrm{~B}-\mathrm{C} 17 \mathrm{~B}-\mathrm{Fe} 2 \mathrm{~B}$ & $120.4(4)$ \\
\hline $\mathrm{C} 25 \mathrm{~A}-\mathrm{C} 16 \mathrm{~A}-\mathrm{C} 17 \mathrm{~A}-\mathrm{C} 18 \mathrm{~A}$ & $-179.6(3)$ & $\mathrm{C} 25 \mathrm{~B}-\mathrm{C} 16 \mathrm{~B}-\mathrm{C} 17 \mathrm{~B}-\mathrm{C} 18 \mathrm{~B}$ & $179.3(3)$ \\
\hline $\mathrm{C} 34 \mathrm{~A}-\mathrm{P} 1 \mathrm{~A}-\mathrm{C} 1 \mathrm{~A}-\mathrm{Fe} 1 \mathrm{~A}$ & $-67.2(2)$ & $\mathrm{C} 34 \mathrm{~B}-\mathrm{P} 1 \mathrm{~B}-\mathrm{C} 1 \mathrm{~B}-\mathrm{Fe} 1 \mathrm{~B}$ & $-64.1(2)$ \\
\hline $\mathrm{C} 34 \mathrm{~A}-\mathrm{P} 1 \mathrm{~A}-\mathrm{C} 1 \mathrm{~A}-\mathrm{C} 2 \mathrm{~A}$ & $-164.3(3)$ & $\mathrm{C} 34 \mathrm{~B}-\mathrm{P} 1 \mathrm{~B}-\mathrm{C} 1 \mathrm{~B}-\mathrm{C} 2 \mathrm{~B}$ & $-161.7(3)$ \\
\hline $\mathrm{C} 34 \mathrm{~A}-\mathrm{P} 1 \mathrm{~A}-\mathrm{C} 1 \mathrm{~A}-\mathrm{C} 5 \mathrm{~A}$ & $23.7(3)$ & $\mathrm{C} 34 \mathrm{~B}-\mathrm{P} 1 \mathrm{~B}-\mathrm{C} 1 \mathrm{~B}-\mathrm{C} 5 \mathrm{~B}$ & $26.9(3)$ \\
\hline $\mathrm{C} 34 \mathrm{~A}-\mathrm{P} 1 \mathrm{~A}-\mathrm{C} 15 \mathrm{~A}-\mathrm{Fe} 2 \mathrm{~A}$ & $168.35(19)$ & $\mathrm{C} 34 \mathrm{~B}-\mathrm{P} 1 \mathrm{~B}-\mathrm{C} 15 \mathrm{~B}-\mathrm{Fe} 2 \mathrm{~B}$ & $168.87(18)$ \\
\hline $\mathrm{C} 34 \mathrm{~A}-\mathrm{P} 1 \mathrm{~A}-\mathrm{C} 15 \mathrm{~A}-\mathrm{C} 16 \mathrm{~A}$ & $70.8(3)$ & $\mathrm{C} 34 \mathrm{~B}-\mathrm{P} 1 \mathrm{~B}-\mathrm{C} 15 \mathrm{~B}-\mathrm{C} 16 \mathrm{~B}$ & $74.6(3)$ \\
\hline $\mathrm{C} 34 \mathrm{~A}-\mathrm{P} 1 \mathrm{~A}-\mathrm{C} 15 \mathrm{~A}-\mathrm{C} 19 \mathrm{~A}$ & $-97.8(3)$ & $\mathrm{C} 34 \mathrm{~B}-\mathrm{P} 1 \mathrm{~B}-\mathrm{C} 15 \mathrm{~B}-\mathrm{C} 19 \mathrm{~B}$ & $-100.7(3)$ \\
\hline $\mathrm{C} 34 \mathrm{~A}-\mathrm{C} 35 \mathrm{~A}-\mathrm{C} 36 \mathrm{~A}-\mathrm{C} 37 \mathrm{~A}$ & $-1.1(5)$ & $\mathrm{C} 34 \mathrm{~B}-\mathrm{C} 35 \mathrm{~B}-\mathrm{C} 36 \mathrm{~B}-\mathrm{C} 37 \mathrm{~B}$ & $1.0(5)$ \\
\hline $\mathrm{C} 35 \mathrm{~A}-\mathrm{C} 34 \mathrm{~A}-\mathrm{C} 39 \mathrm{~A}-\mathrm{C} 38 \mathrm{~A}$ & $-1.3(4)$ & $\mathrm{C} 35 \mathrm{~B}-\mathrm{C} 34 \mathrm{~B}-\mathrm{C} 39 \mathrm{~B}-\mathrm{C} 38 \mathrm{~B}$ & $1.5(5)$ \\
\hline $\mathrm{C} 35 \mathrm{~A}-\mathrm{C} 36 \mathrm{~A}-\mathrm{C} 37 \mathrm{~A}-\mathrm{C} 38 \mathrm{~A}$ & $-0.6(5)$ & $\mathrm{C} 35 \mathrm{~B}-\mathrm{C} 36 \mathrm{~B}-\mathrm{C} 37 \mathrm{~B}-\mathrm{C} 38 \mathrm{~B}$ & $0.0(5)$ \\
\hline $\mathrm{C} 36 \mathrm{~A}-\mathrm{C} 37 \mathrm{~A}-\mathrm{C} 38 \mathrm{~A}-\mathrm{C} 39 \mathrm{~A}$ & $1.3(5)$ & $\mathrm{C} 36 \mathrm{~B}-\mathrm{C} 37 \mathrm{~B}-\mathrm{C} 38 \mathrm{~B}-\mathrm{C} 39 \mathrm{~B}$ & $-0.3(5)$ \\
\hline $\mathrm{C} 37 \mathrm{~A}-\mathrm{C} 38 \mathrm{~A}-\mathrm{C} 39 \mathrm{~A}-\mathrm{C} 34 \mathrm{~A}$ & $-0.4(5)$ & $\mathrm{C} 37 \mathrm{~B}-\mathrm{C} 38 \mathrm{~B}-\mathrm{C} 39 \mathrm{~B}-\mathrm{C} 34 \mathrm{~B}$ & $-0.5(5)$ \\
\hline $\mathrm{C} 39 \mathrm{~A}-\mathrm{C} 34 \mathrm{~A}-\mathrm{C} 35 \mathrm{~A}-\mathrm{C} 36 \mathrm{~A}$ & $2.0(4)$ & $\mathrm{C} 39 \mathrm{~B}-\mathrm{C} 34 \mathrm{~B}-\mathrm{C} 35 \mathrm{~B}-\mathrm{C} 36 \mathrm{~B}$ & $-1.7(5)$ \\
\hline
\end{tabular}

1,1"-(Phenylphosphanediyl)bis $\left\{\left(2 S_{\mathrm{p}}\right)-2-[(1 R)-1-\backslash\right.$ (acetyloxy)ethyl]ferrocene $\}$ (7)

Crystal data

$\left[\mathrm{Fe}_{2}\left(\mathrm{C}_{5} \mathrm{H}_{5}\right)_{2}\left(\mathrm{C}_{24} \mathrm{H}_{25} \mathrm{O}_{4} \mathrm{P}\right)\right]$

$M_{r}=650.29$

Orthorhombic, $P 22_{1} 2_{1} 2_{1}$

$a=7.631(2) \AA$

$b=10.877(2) \AA$

$c=36.025(8) \AA$
$V=2990.2(12) \AA^{3}$

$Z=4$

$F(000)=1352$

$D_{\mathrm{x}}=1.444 \mathrm{Mg} \mathrm{m}^{-3}$

Mo $K \alpha$ radiation, $\lambda=0.71073 \AA$

Cell parameters from 3094 reflections 
$\theta=2.2-19.9^{\circ}$

$\mu=1.06 \mathrm{~mm}^{-1}$

$T=130 \mathrm{~K}$

\section{Data collection}

\section{Bruker X8 APEXII}

diffractometer

Radiation source: sealed xray tube, Incoatec IuS

$\varphi$ and $\omega$ scans

Absorption correction: multi-scan

(SADABS; Bruker, 2008)

$T_{\min }=0.562, T_{\max }=0.745$

46099 measured reflections

\section{Refinement}

Refinement on $F^{2}$

Least-squares matrix: full

$R\left[F^{2}>2 \sigma\left(F^{2}\right)\right]=0.065$

$w R\left(F^{2}\right)=0.122$

$S=1.04$

5441 reflections

374 parameters

0 restraints

Primary atom site location: structure-invariant direct methods
Block, clear orange

$0.15 \times 0.08 \times 0.06 \mathrm{~mm}$

5441 independent reflections

4085 reflections with $I>2 \sigma(I)$

$R_{\text {int }}=0.150$

$\theta_{\max }=25.4^{\circ}, \theta_{\min }=2.2^{\circ}$

$h=-9 \rightarrow 9$

$k=-13 \rightarrow 13$

$l=-38 \rightarrow 43$

Hydrogen site location: inferred from neighbouring sites

$\mathrm{H}$-atom parameters constrained

$w=1 /\left[\sigma^{2}\left(F_{\mathrm{o}}^{2}\right)+(0.0421 P)^{2}+2.7228 P\right]$

where $P=\left(F_{\mathrm{o}}^{2}+2 F_{\mathrm{c}}^{2}\right) / 3$

$(\Delta / \sigma)_{\max }<0.001$

$\Delta \rho_{\max }=0.94$ e $\AA^{-3}$

$\Delta \rho_{\text {min }}=-0.43$ e $\AA^{-3}$

Absolute structure: Flack $x$ determined using 1235 quotients $[(\mathrm{I}+)-(\mathrm{I}-)] /[(\mathrm{I}+)+(\mathrm{I}-)]$ (Parsons et al., 2013)

Absolute structure parameter: -0.02 (2)

\section{Special details}

Geometry. All esds (except the esd in the dihedral angle between two 1.s. planes) are estimated using the full covariance matrix. The cell esds are taken into account individually in the estimation of esds in distances, angles and torsion angles; correlations between esds in cell parameters are only used when they are defined by crystal symmetry. An approximate (isotropic) treatment of cell esds is used for estimating esds involving l.s. planes.

Refinement._olex2_refinement_description

1. Fixed Uiso At 1.2 times of: All $\mathrm{C}(\mathrm{H})$ groups At 1.5 times of: All $\mathrm{C}(\mathrm{H}, \mathrm{H}, \mathrm{H})$ groups 2.a Ternary $\mathrm{CH}$ refined with riding coordinates: $\mathrm{C} 11(\mathrm{H} 11), \mathrm{C} 25(\mathrm{H} 25)$ 2.b Aromatic/amide $\mathrm{H}$ refined with riding coordinates: $\mathrm{C} 3(\mathrm{H} 3), \mathrm{C} 4(\mathrm{H} 4)$, C5(H5), C6(H6), C7(H7), C8(H8), C9(H9), C10(H10), C17(H17), C18(H18), C19(H19), C20(H20), C21(H21), C22(H22), C23(H23), C24(H24), C35(H35), C36(H36), C37(H37), C38(H38), C39(H39) 2.c Idealised Me refined as rotating group: C13(H13A,H13B,H13C), C14(H14A,H14B,H14C), C27(H27A,H27B,H27C), C28(H28A,H28B, H28C)

Fractional atomic coordinates and isotropic or equivalent isotropic displacement parameters $\left(\AA^{2}\right)$

\begin{tabular}{lllll}
\hline & $x$ & $y$ & $z$ & $U_{\text {iso }} * / U_{\text {eq }}$ \\
\hline Fe1 & $0.79439(15)$ & $0.89449(11)$ & $0.38276(3)$ & $0.0264(3)$ \\
Fe2 & $1.24405(15)$ & $0.40848(10)$ & $0.40108(3)$ & $0.0235(3)$ \\
P1 & $0.8827(3)$ & $0.5855(2)$ & $0.37276(5)$ & $0.0218(5)$ \\
O1 & $0.7061(8)$ & $0.7131(5)$ & $0.27691(15)$ & $0.0339(15)$ \\
O2 & $0.4850(9)$ & $0.5800(7)$ & $0.26629(19)$ & $0.0519(19)$ \\
O3 & $0.8911(7)$ & $0.5751(5)$ & $0.48204(14)$ & $0.0275(14)$ \\
O4 & $0.6319(8)$ & $0.4892(6)$ & $0.49674(18)$ & $0.0430(18)$ \\
C1 & $0.9068(11)$ & $0.7452(7)$ & $0.3578(2)$ & $0.020(2)$ \\
C2 & $0.7857(12)$ & $0.8062(7)$ & $0.3331(2)$ & $0.026(2)$ \\
C3 & $0.8444(12)$ & $0.9294(8)$ & $0.3278(2)$ & $0.033(2)$
\end{tabular}




\begin{tabular}{|c|c|c|c|c|}
\hline $\mathrm{H} 3$ & 0.790570 & 0.989466 & 0.312382 & $0.039^{*}$ \\
\hline $\mathrm{C} 4$ & $0.9979(12)$ & $0.9465(8)$ & $0.3496(2)$ & $0.031(2)$ \\
\hline H4 & 1.061986 & 1.021088 & 0.351643 & $0.037 *$ \\
\hline $\mathrm{C} 5$ & $1.0401(12)$ & $0.8347(8)$ & $0.3678(2)$ & $0.026(2)$ \\
\hline H5 & 1.137758 & 0.820808 & 0.383551 & $0.032 *$ \\
\hline C6 & $0.7112(15)$ & $0.8428(9)$ & $0.4348(3)$ & $0.044(3)$ \\
\hline H6 & 0.740860 & 0.768806 & 0.447365 & $0.053^{*}$ \\
\hline $\mathrm{C} 7$ & $0.5651(14)$ & $0.8616(10)$ & $0.4100(3)$ & $0.044(3)$ \\
\hline H7 & 0.480269 & 0.801654 & 0.403352 & $0.053 *$ \\
\hline $\mathrm{C} 8$ & $0.5702(12)$ & $0.9839(8)$ & $0.3975(3)$ & $0.034(2)$ \\
\hline H8 & 0.489830 & 1.020809 & 0.380758 & $0.041^{*}$ \\
\hline C9 & $0.7151(12)$ & $1.0423(8)$ & $0.4141(2)$ & $0.033(2)$ \\
\hline H9 & 0.749057 & 1.125501 & 0.410609 & $0.040 *$ \\
\hline $\mathrm{C} 10$ & $0.8010(15)$ & $0.9556(10)$ & $0.4368(2)$ & $0.050(3)$ \\
\hline H10 & 0.903184 & 0.970965 & 0.451170 & $0.060^{*}$ \\
\hline $\mathrm{C} 11$ & $0.6349(12)$ & $0.7474(8)$ & $0.3133(2)$ & $0.029(2)$ \\
\hline H11 & 0.598509 & 0.671501 & 0.326949 & $0.035^{*}$ \\
\hline $\mathrm{C} 12$ & $0.6172(13)$ & $0.6303(8)$ & $0.2565(3)$ & $0.036(2)$ \\
\hline $\mathrm{C} 13$ & $0.7073(15)$ & $0.6108(9)$ & $0.2201(2)$ & $0.055(3)$ \\
\hline $\mathrm{H} 13 \mathrm{~A}$ & 0.785645 & 0.680231 & 0.215101 & $0.082 *$ \\
\hline H13B & 0.619605 & 0.604836 & 0.200333 & $0.082 *$ \\
\hline $\mathrm{H} 13 \mathrm{C}$ & 0.775670 & 0.534615 & 0.221092 & $0.082 *$ \\
\hline C14 & $0.4754(13)$ & $0.8309(9)$ & 0.3079 (3) & $0.043(3)$ \\
\hline H14A & 0.506901 & 0.899428 & 0.291501 & $0.064^{*}$ \\
\hline H14B & 0.437420 & 0.863080 & 0.331953 & $0.064^{*}$ \\
\hline $\mathrm{H} 14 \mathrm{C}$ & 0.379809 & 0.783626 & 0.296612 & $0.064 *$ \\
\hline C15 & $1.0846(10)$ & $0.5607(7)$ & $0.3990(2)$ & $0.0198(19)$ \\
\hline $\mathrm{C} 16$ & $1.0900(11)$ & $0.5102(8)$ & $0.4361(2)$ & $0.021(2)$ \\
\hline $\mathrm{C} 17$ & $1.2691(13)$ & $0.5118(8)$ & $0.4482(2)$ & $0.032(2)$ \\
\hline H17 & 1.310570 & 0.484611 & 0.471699 & $0.038^{*}$ \\
\hline $\mathrm{C} 18$ & $1.3738(12)$ & $0.5607(8)$ & $0.4194(2)$ & $0.029(2)$ \\
\hline H18 & 1.497201 & 0.571824 & 0.420079 & $0.035^{*}$ \\
\hline $\mathrm{C} 19$ & $1.2615(11)$ & $0.5902(7)$ & $0.3889(2)$ & $0.0265(18)$ \\
\hline H19 & 1.297952 & 0.623840 & 0.365846 & $0.032 *$ \\
\hline $\mathrm{C} 20$ & $1.1716(14)$ & $0.2763(8)$ & $0.3636(3)$ & $0.038(3)$ \\
\hline $\mathrm{H} 20$ & 1.073876 & 0.281040 & 0.347373 & $0.045^{*}$ \\
\hline $\mathrm{C} 21$ & $1.1677(14)$ & $0.2267(8)$ & $0.3999(3)$ & 0.037 (3) \\
\hline H21 & 1.069405 & 0.191373 & 0.412034 & $0.044^{*}$ \\
\hline $\mathrm{C} 22$ & $1.3408(14)$ & $0.2401(9)$ & $0.4146(3)$ & $0.035(3)$ \\
\hline $\mathrm{H} 22$ & 1.378716 & 0.215886 & 0.438661 & $0.042 *$ \\
\hline $\mathrm{C} 23$ & $1.4448(13)$ & $0.2959(8)$ & $0.3869(3)$ & $0.036(2)$ \\
\hline $\mathrm{H} 23$ & 1.565586 & 0.315609 & 0.389227 & $0.043^{*}$ \\
\hline $\mathrm{C} 24$ & $1.3406(13)$ & $0.3175(8)$ & $0.3553(3)$ & $0.033(2)$ \\
\hline $\mathrm{H} 24$ & 1.378053 & 0.353351 & 0.332601 & $0.039 *$ \\
\hline $\mathrm{C} 25$ & $0.9380(12)$ & $0.4680(7)$ & $0.4591(2)$ & $0.022(2)$ \\
\hline $\mathrm{H} 25$ & 0.837562 & 0.444799 & 0.442727 & $0.027 *$ \\
\hline $\mathrm{C} 26$ & $0.7380(13)$ & $0.5720(8)$ & $0.4997(2)$ & $0.031(2)$ \\
\hline $\mathrm{C} 27$ & $0.7112(14)$ & $0.6845(8)$ & $0.5235(2)$ & $0.043(3)$ \\
\hline
\end{tabular}


supporting information

$\begin{array}{lllll}\text { H27A } & 0.816621 & 0.735870 & 0.522558 & 0.064^{*} \\ \text { H27B } & 0.688940 & 0.659261 & 0.549145 & 0.064^{*} \\ \text { H27C } & 0.610816 & 0.731348 & 0.514134 & 0.064^{*} \\ \text { C28 } & 0.9831(14) & 0.3608(9) & 0.4855(3) & 0.044(3) \\ \text { H28A } & 1.065265 & 0.389470 & 0.504559 & 0.065^{*} \\ \text { H28B } & 1.037004 & 0.293848 & 0.471332 & 0.065^{*} \\ \text { H28C } & 0.875797 & 0.330975 & 0.497440 & 0.065^{*} \\ \text { C34 } & 0.9200(11) & 0.5057(7) & 0.3283(2) & 0.0196(19) \\ \text { C35 } & 0.7961(11) & 0.4143(8) & 0.3191(2) & 0.033(2) \\ \text { H35 } & 0.697616 & 0.400786 & 0.334657 & 0.039^{*} \\ \text { C36 } & 0.8189(14) & 0.3433(8) & 0.2867(2) & 0.038(3) \\ \text { H36 } & 0.734927 & 0.282046 & 0.280597 & 0.046^{*} \\ \text { C37 } & 0.9591(13) & 0.3611(9) & 0.2643(3) & 0.039(3) \\ \text { H37 } & 0.974890 & 0.311589 & 0.242803 & 0.047^{*} \\ \text { C38 } & 1.0812(12) & 0.4537(9) & 0.2730(2) & 0.036(2) \\ \text { H38 } & 1.179345 & 0.467063 & 0.257293 & 0.043^{*} \\ \text { C39 } & 1.0589(11) & 0.5252(8) & 0.3043(2) & 0.024(2) \\ \text { H39 } & 1.140352 & 0.589012 & 0.309469 & 0.029^{*}\end{array}$

Atomic displacement parameters $\left(\AA^{2}\right)$

\begin{tabular}{|c|c|c|c|c|c|c|}
\hline & $U^{11}$ & $U^{22}$ & $U^{33}$ & $U^{12}$ & $U^{13}$ & $U^{23}$ \\
\hline $\mathrm{Fe} 1$ & $0.0298(7)$ & $0.0221(6)$ & $0.0273(7)$ & $0.0056(6)$ & $0.0064(5)$ & $0.0017(6)$ \\
\hline $\mathrm{Fe} 2$ & $0.0249(6)$ & $0.0214(6)$ & $0.0243(6)$ & $0.0049(6)$ & $-0.0004(5)$ & $-0.0025(5)$ \\
\hline P1 & $0.0228(11)$ & $0.0202(11)$ & $0.0224(12)$ & $0.0041(10)$ & $0.0004(9)$ & $0.0004(10)$ \\
\hline $\mathrm{O} 1$ & 0.043 (4) & $0.035(3)$ & $0.024(3)$ & $-0.008(3)$ & $-0.002(3)$ & $0.000(3)$ \\
\hline $\mathrm{O} 2$ & $0.048(5)$ & $0.050(5)$ & $0.058(5)$ & -0.009 (4) & -0.003 (4) & $0.002(4)$ \\
\hline $\mathrm{O} 3$ & $0.027(3)$ & $0.031(4)$ & $0.024(3)$ & $0.004(3)$ & 0.009 (3) & $-0.008(3)$ \\
\hline $\mathrm{O} 4$ & $0.036(4)$ & $0.050(4)$ & $0.043(4)$ & $-0.013(4)$ & $0.006(3)$ & $-0.011(4)$ \\
\hline $\mathrm{C} 1$ & $0.024(5)$ & $0.014(4)$ & $0.022(5)$ & $0.004(4)$ & $0.005(4)$ & 0.006 (4) \\
\hline $\mathrm{C} 2$ & $0.029(6)$ & $0.020(4)$ & $0.030(5)$ & $0.006(4)$ & $0.001(4)$ & $0.001(4)$ \\
\hline $\mathrm{C} 3$ & $0.043(6)$ & $0.033(6)$ & $0.022(5)$ & $0.005(5)$ & $0.010(4)$ & $0.003(4)$ \\
\hline $\mathrm{C} 4$ & $0.040(6)$ & $0.022(5)$ & $0.031(6)$ & -0.001 & $0.011(4)$ & $0.001(4)$ \\
\hline $\mathrm{C} 5$ & $0.022(5)$ & $0.024(5)$ & $0.032(6)$ & -0.003 & $0.010(4)$ & -0.001 \\
\hline C6 & $0.065(9)$ & $0.033(6)$ & $0.034(6)$ & $0.019(6)$ & $0.025(6)$ & $0.013(5)$ \\
\hline $\mathrm{C} 7$ & $0.040(7)$ & $0.043(6)$ & $0.050(7)$ & $-0.003(5)$ & $0.026(5)$ & $-0.009(5)$ \\
\hline $\mathrm{C} 8$ & $0.029(5)$ & $0.028(5)$ & $0.045(6)$ & $0.017(4)$ & $0.003(5)$ & $-0.010(5)$ \\
\hline C9 & $0.030(6)$ & $0.028(5)$ & $0.043(6)$ & $0.005(4)$ & $0.005(5)$ & -0.004 (4) \\
\hline $\mathrm{C} 10$ & $0.054(7)$ & $0.071(8)$ & $0.024(5)$ & $0.030(6)$ & $0.004(5)$ & $-0.010(5)$ \\
\hline $\mathrm{C} 11$ & $0.030(6)$ & $0.024(5)$ & $0.034(6)$ & 0.005 & $-0.004(4)$ & $0.004(4)$ \\
\hline $\mathrm{C} 12$ & $0.037(6)$ & $0.034(6)$ & $0.037(6)$ & $-0.008(5)$ & $-0.007(5)$ & $0.005(5)$ \\
\hline $\mathrm{C} 13$ & $0.085(8)$ & $0.041(6)$ & $0.039(6)$ & $-0.027(7)$ & $-0.004(6)$ & $0.002(5)$ \\
\hline $\mathrm{C} 14$ & $0.032(6)$ & $0.051(7)$ & $0.045(7)$ & $0.001(5)$ & $-0.007(5)$ & $0.012(5)$ \\
\hline $\mathrm{C} 15$ & $0.020(5)$ & $0.018(5)$ & $0.022(5)$ & $0.004(3)$ & $0.003(4)$ & $-0.006(4)$ \\
\hline $\mathrm{C} 16$ & $0.028(5)$ & $0.019(5)$ & $0.016(5)$ & 0.007 (4) & $-0.004(4)$ & $-0.004(4)$ \\
\hline $\mathrm{C} 17$ & $0.044(6)$ & $0.031(5)$ & $0.020(5)$ & $0.010(5)$ & -0.009 (5) & $0.000(4)$ \\
\hline $\mathrm{C} 18$ & $0.019(5)$ & $0.026(5)$ & $0.043(6)$ & $0.003(4)$ & $-0.001(4)$ & -0.005 (4) \\
\hline C19 & $0.033(5)$ & $0.015(4)$ & $0.032(5)$ & -0.002 & $0.006(4)$ & -0.003 \\
\hline
\end{tabular}




$\begin{array}{lllllll}\mathrm{C} 20 & 0.056(7) & 0.025(5) & 0.032(6) & 0.009(5) & -0.005(5) & -0.015(4) \\ \text { C21 } & 0.046(7) & 0.022(5) & 0.042(6) & -0.001(4) & 0.020(5) & -0.010(5) \\ \text { C22 } & 0.053(8) & 0.028(5) & 0.025(5) & 0.011(5) & 0.002(5) & -0.004(4) \\ \text { C23 } & 0.032(6) & 0.036(6) & 0.040(6) & 0.013(5) & 0.002(5) & -0.001(5) \\ \text { C24 } & 0.044(7) & 0.022(5) & 0.033(5) & 0.003(4) & 0.000(5) & -0.002(4) \\ \text { C25 } & 0.033(5) & 0.019(5) & 0.014(5) & 0.003(4) & -0.001(4) & -0.002(4) \\ \text { C26 } & 0.035(6) & 0.035(5) & 0.023(5) & 0.004(5) & -0.009(4) & -0.005(4) \\ \text { C27 } & 0.043(7) & 0.049(6) & 0.037(6) & 0.010(5) & 0.004(5) & -0.009(5) \\ \text { C28 } & 0.061(8) & 0.036(6) & 0.034(6) & 0.013(5) & 0.008(5) & 0.014(5) \\ \text { C34 } & 0.026(5) & 0.022(5) & 0.012(4) & 0.005(4) & -0.005(4) & 0.000(4) \\ \text { C35 } & 0.029(5) & 0.042(5) & 0.027(5) & -0.011(5) & -0.005(4) & 0.002(5) \\ \text { C36 } & 0.044(7) & 0.035(5) & 0.037(6) & -0.009(5) & -0.006(5) & -0.006(5) \\ \text { C37 } & 0.044(7) & 0.046(6) & 0.028(6) & 0.009(5) & -0.004(5) & -0.016(5) \\ \text { C38 } & 0.029(6) & 0.052(7) & 0.027(5) & 0.005(5) & 0.003(4) & 0.001(5) \\ \text { C39 } & 0.020(5) & 0.034(5) & 0.019(5) & -0.001(4) & -0.007(4) & 0.001(4)\end{array}$

Geometric parameters $\left(A,{ }^{\circ}\right)$

\begin{tabular}{llll}
\hline $\mathrm{Fe} 1-\mathrm{C} 1$ & $2.044(8)$ & $\mathrm{C} 11-\mathrm{H} 11$ & 1.0000 \\
$\mathrm{Fe} 1-\mathrm{C} 2$ & $2.032(8)$ & $\mathrm{C} 11-\mathrm{C} 14$ & $1.531(12)$ \\
$\mathrm{Fe} 1-\mathrm{C} 3$ & $2.053(8)$ & $\mathrm{C} 12-\mathrm{C} 13$ & $1.496(13)$ \\
$\mathrm{Fe} 1-\mathrm{C} 4$ & $2.040(9)$ & $\mathrm{C} 13-\mathrm{H} 13 \mathrm{~A}$ & 0.9800 \\
$\mathrm{Fe} 1-\mathrm{C} 5$ & $2.057(9)$ & $\mathrm{C} 13-\mathrm{H} 13 \mathrm{~B}$ & 0.9800 \\
$\mathrm{Fe} 1-\mathrm{C} 6$ & $2.059(9)$ & $\mathrm{C} 13-\mathrm{H} 13 \mathrm{C}$ & 0.9800 \\
$\mathrm{Fe} 1-\mathrm{C} 7$ & $2.038(10)$ & $\mathrm{C} 14-\mathrm{H} 14 \mathrm{~A}$ & 0.9800 \\
$\mathrm{Fe} 1-\mathrm{C} 8$ & $2.038(8)$ & $\mathrm{C} 14-\mathrm{H} 14 \mathrm{~B}$ & 0.9800 \\
$\mathrm{Fe} 1-\mathrm{C} 9$ & $2.056(8)$ & $\mathrm{C} 14-\mathrm{H} 14 \mathrm{C}$ & 0.9800 \\
$\mathrm{Fe} 1-\mathrm{C} 10$ & $2.059(9)$ & $\mathrm{C} 15-\mathrm{C} 16$ & $1.446(11)$ \\
$\mathrm{Fe} 2-\mathrm{C} 15$ & $2.056(8)$ & $\mathrm{C} 15-\mathrm{C} 19$ & $1.434(11)$ \\
$\mathrm{Fe} 2-\mathrm{C} 16$ & $2.050(8)$ & $\mathrm{C} 16-\mathrm{C} 17$ & $1.435(12)$ \\
$\mathrm{Fe} 2-\mathrm{C} 17$ & $2.046(8)$ & $\mathrm{C} 16-\mathrm{C} 25$ & $1.497(12)$ \\
$\mathrm{Fe} 2-\mathrm{C} 18$ & $2.038(9)$ & $\mathrm{C} 17-\mathrm{H} 17$ & 0.9500 \\
$\mathrm{Fe} 2-\mathrm{C} 19$ & $2.029(8)$ & $\mathrm{C} 17-\mathrm{C} 18$ & $1.415(12)$ \\
$\mathrm{Fe} 2-\mathrm{C} 20$ & $2.047(9)$ & $\mathrm{C} 18-\mathrm{H} 18$ & 0.9500 \\
$\mathrm{Fe} 2-\mathrm{C} 21$ & $2.062(9)$ & $\mathrm{C} 18-\mathrm{C} 19$ & $1.428(11)$ \\
$\mathrm{Fe} 2-\mathrm{C} 22$ & $2.034(10)$ & $\mathrm{C} 19-\mathrm{H} 19$ & 0.9500 \\
$\mathrm{Fe} 2-\mathrm{C} 23$ & $2.027(9)$ & $\mathrm{C} 20-\mathrm{H} 20$ & 0.9500 \\
$\mathrm{Fe} 2-\mathrm{C} 24$ & $2.059(9)$ & $\mathrm{C} 20-\mathrm{C} 21$ & $1.412(12)$ \\
$\mathrm{P} 1-\mathrm{C} 1$ & $1.828(8)$ & $\mathrm{C} 20-\mathrm{C} 24$ & $1.398(13)$ \\
$\mathrm{P} 1-\mathrm{C} 15$ & $1.828(8)$ & $\mathrm{C} 21-\mathrm{H} 21$ & 0.9500 \\
$\mathrm{P} 1-\mathrm{C} 34$ & $1.845(8)$ & $\mathrm{C} 21-\mathrm{C} 22$ & $1.432(13)$ \\
$\mathrm{O} 1-\mathrm{C} 11$ & $1.468(10)$ & $\mathrm{C} 22-\mathrm{H} 22$ & 0.9500 \\
$\mathrm{O} 1-\mathrm{C} 12$ & $1.345(10)$ & $\mathrm{C} 22-\mathrm{C} 23$ & $1.412(13)$ \\
$\mathrm{O} 2-\mathrm{C} 12$ & $1.200(10)$ & $\mathrm{C} 23-\mathrm{H} 23$ & 0.9500 \\
$\mathrm{O} 3-\mathrm{C} 25$ & $1.472(9)$ & $\mathrm{C} 23-\mathrm{C} 24$ & $1.409(12)$ \\
$\mathrm{O} 3-\mathrm{C} 26$ & $1.331(10)$ & $\mathrm{C} 24-\mathrm{H} 24$ & 1.0000 \\
$\mathrm{O} 4-\mathrm{C} 26$ & $1.216(10)$ & $\mathrm{C} 25-\mathrm{H} 25$ & $\mathrm{C} 25-\mathrm{C} 28$ \\
$\mathrm{C} 1-\mathrm{C} 2$ & $1.446(11)$ & &
\end{tabular}




\begin{tabular}{|c|c|c|c|}
\hline $\mathrm{C} 1-\mathrm{C} 5$ & $1.452(12)$ & $\mathrm{C} 26-\mathrm{C} 27$ & $1.508(11)$ \\
\hline $\mathrm{C} 2-\mathrm{C} 3$ & $1.426(12)$ & $\mathrm{C} 27-\mathrm{H} 27 \mathrm{~A}$ & 0.9800 \\
\hline $\mathrm{C} 2-\mathrm{C} 11$ & 1.497 (12) & $\mathrm{C} 27-\mathrm{H} 27 \mathrm{~B}$ & 0.9800 \\
\hline $\mathrm{C} 3-\mathrm{H} 3$ & 0.9500 & $\mathrm{C} 27-\mathrm{H} 27 \mathrm{C}$ & 0.9800 \\
\hline $\mathrm{C} 3-\mathrm{C} 4$ & $1.422(12)$ & $\mathrm{C} 28-\mathrm{H} 28 \mathrm{~A}$ & 0.9800 \\
\hline $\mathrm{C} 4-\mathrm{H} 4$ & 0.9500 & $\mathrm{C} 28-\mathrm{H} 28 \mathrm{~B}$ & 0.9800 \\
\hline $\mathrm{C} 4-\mathrm{C} 5$ & $1.419(11)$ & $\mathrm{C} 28-\mathrm{H} 28 \mathrm{C}$ & 0.9800 \\
\hline $\mathrm{C} 5-\mathrm{H} 5$ & 0.9500 & C34-C35 & $1.411(11)$ \\
\hline $\mathrm{C} 6-\mathrm{H} 6$ & 0.9500 & C34-C39 & $1.383(11)$ \\
\hline $\mathrm{C} 6-\mathrm{C} 7$ & $1.443(14)$ & C $35-\mathrm{H} 35$ & 0.9500 \\
\hline $\mathrm{C} 6-\mathrm{C} 10$ & $1.408(14)$ & $\mathrm{C} 35-\mathrm{C} 36$ & $1.409(12)$ \\
\hline $\mathrm{C} 7-\mathrm{H} 7$ & 0.9500 & C36-H36 & 0.9500 \\
\hline $\mathrm{C} 7-\mathrm{C} 8$ & $1.406(13)$ & $\mathrm{C} 36-\mathrm{C} 37$ & $1.356(13)$ \\
\hline $\mathrm{C} 8-\mathrm{H} 8$ & 0.9500 & C37-H37 & 0.9500 \\
\hline $\mathrm{C} 8-\mathrm{C} 9$ & $1.408(13)$ & $\mathrm{C} 37-\mathrm{C} 38$ & $1.407(13)$ \\
\hline C9- & 0.9500 & C $38-\mathrm{H} 38$ & 0.9500 \\
\hline $\mathrm{C} 9-\mathrm{C} 10$ & $1.410(12)$ & $\mathrm{C} 38-\mathrm{C} 39$ & $1.380(12)$ \\
\hline $\mathrm{C} 10-\mathrm{H} 10$ & 0.9500 & C39-H39 & 0.9500 \\
\hline $\mathrm{C} 1-\mathrm{Fe} 1-\mathrm{C} 3$ & $69.2(3)$ & $\mathrm{C} 7-\mathrm{C} 8-\mathrm{Fe} 1$ & $69.8(5)$ \\
\hline $\mathrm{C} 1-\mathrm{Fe} 1-\mathrm{C} 5$ & $41.5(3)$ & $\mathrm{C} 7-\mathrm{C} 8-\mathrm{H} 8$ & 125.9 \\
\hline $\mathrm{C} 1-\mathrm{Fe} 1-\mathrm{C} 6$ & $108.2(3)$ & $\mathrm{C} 7-\mathrm{C} 8-\mathrm{C} 9$ & $108.2(9)$ \\
\hline $\mathrm{C} 1-\mathrm{Fe} 1-\mathrm{C} 9$ & $170.4(4)$ & $\mathrm{C} 9-\mathrm{C} 8-\mathrm{Fe} 1$ & $70.5(5)$ \\
\hline $\mathrm{C} 1-\mathrm{Fe} 1-\mathrm{C} 10$ & $131.4(4)$ & $\mathrm{C} 9-\mathrm{C} 8-\mathrm{H} 8$ & 125.9 \\
\hline $\mathrm{C} 2-\mathrm{Fe} 1-\mathrm{C} 1$ & $41.5(3)$ & $\mathrm{Fe} 1-\mathrm{C} 9-\mathrm{H} 9$ & 126.3 \\
\hline $\mathrm{C} 2-\mathrm{Fe} 1-\mathrm{C} 3$ & $40.9(3)$ & $\mathrm{C} 8-\mathrm{C} 9-\mathrm{Fe} 1$ & $69.2(5)$ \\
\hline $\mathrm{C} 2-\mathrm{Fe} 1-\mathrm{C} 4$ & $68.9(4)$ & $\mathrm{C} 8-\mathrm{C} 9-\mathrm{H} 9$ & 126.0 \\
\hline $\mathrm{C} 2-\mathrm{Fe} 1-\mathrm{C} 5$ & $69.4(4)$ & $\mathrm{C} 8-\mathrm{C} 9-\mathrm{C} 10$ & $108.1(9)$ \\
\hline $\mathrm{C} 2-\mathrm{Fe} 1-\mathrm{C} 6$ & $131.6(4)$ & $\mathrm{C} 10-\mathrm{C} 9-\mathrm{Fe} 1$ & $70.1(5)$ \\
\hline $\mathrm{C} 2-\mathrm{Fe} 1-\mathrm{C} 7$ & $108.3(4)$ & $\mathrm{C} 10-\mathrm{C} 9-\mathrm{H} 9$ & 126.0 \\
\hline $\mathrm{C} 2-\mathrm{Fe} 1-\mathrm{C} 8$ & $115.3(4)$ & $\mathrm{Fe} 1-\mathrm{C} 10-\mathrm{H} 10$ & 126.3 \\
\hline $\mathrm{C} 2-\mathrm{Fe} 1-\mathrm{C} 9$ & $147.5(3)$ & $\mathrm{C} 6-\mathrm{C} 10-\mathrm{Fe} 1$ & $70.0(5)$ \\
\hline $\mathrm{C} 2-\mathrm{Fe} 1-\mathrm{C} 10$ & $170.6(4)$ & $\mathrm{C} 6-\mathrm{C} 10-\mathrm{C} 9$ & $109.1(9)$ \\
\hline $\mathrm{C} 3-\mathrm{Fe} 1-\mathrm{C} 5$ & $68.6(4)$ & $\mathrm{C} 6-\mathrm{C} 10-\mathrm{H} 10$ & 125.5 \\
\hline $\mathrm{C} 3-\mathrm{Fe} 1-\mathrm{C} 6$ & $170.8(4)$ & $\mathrm{C} 9-\mathrm{C} 10-\mathrm{Fe} 1$ & $69.8(5)$ \\
\hline $\mathrm{C} 3-\mathrm{Fe} 1-\mathrm{C} 9$ & $116.1(4)$ & $\mathrm{C} 9-\mathrm{C} 10-\mathrm{H} 10$ & 125.5 \\
\hline $\mathrm{C} 3-\mathrm{Fe} 1-\mathrm{C} 10$ & $148.1(4)$ & $\mathrm{O} 1-\mathrm{C} 11-\mathrm{C} 2$ & $104.4(7)$ \\
\hline $\mathrm{C} 4-\mathrm{Fe} 1-\mathrm{C} 1$ & $69.1(3)$ & $\mathrm{O} 1-\mathrm{C} 11-\mathrm{H} 11$ & 109.4 \\
\hline $\mathrm{C} 4-\mathrm{Fe} 1-\mathrm{C} 3$ & $40.7(3)$ & $\mathrm{O} 1-\mathrm{C} 11-\mathrm{C} 14$ & $109.3(7)$ \\
\hline $\mathrm{C} 4-\mathrm{Fe} 1-\mathrm{C} 5$ & $40.5(3)$ & $\mathrm{C} 2-\mathrm{C} 11-\mathrm{H} 11$ & 109.4 \\
\hline $\mathrm{C} 4-\mathrm{Fe} 1-\mathrm{C} 6$ & $147.6(4)$ & $\mathrm{C} 2-\mathrm{C} 11-\mathrm{C} 14$ & $114.7(7)$ \\
\hline $\mathrm{C} 4-\mathrm{Fe} 1-\mathrm{C} 9$ & $109.2(4)$ & $\mathrm{C} 14-\mathrm{C} 11-\mathrm{H} 11$ & 109.4 \\
\hline $\mathrm{C} 4-\mathrm{Fe} 1-\mathrm{C} 10$ & $116.5(4)$ & $\mathrm{O} 1-\mathrm{C} 12-\mathrm{C} 13$ & $110.0(8)$ \\
\hline $\mathrm{C} 5-\mathrm{Fe} 1-\mathrm{C} 6$ & $115.7(4)$ & $\mathrm{O} 2-\mathrm{C} 12-\mathrm{O} 1$ & $124.7(9)$ \\
\hline $\mathrm{C} 5-\mathrm{Fe} 1-\mathrm{C} 10$ & $109.2(4)$ & $\mathrm{O} 2-\mathrm{C} 12-\mathrm{C} 13$ & $125.4(9)$ \\
\hline $\mathrm{C} 7-\mathrm{Fe} 1-\mathrm{C} 1$ & $115.6(4)$ & $\mathrm{C} 12-\mathrm{C} 13-\mathrm{H} 13 \mathrm{~A}$ & 109.5 \\
\hline $\mathrm{C} 7-\mathrm{Fe} 1-\mathrm{C} 3$ & $131.1(4)$ & $\mathrm{C} 12-\mathrm{C} 13-\mathrm{H} 13 \mathrm{~B}$ & 109.5 \\
\hline $\mathrm{C} 7-\mathrm{Fe} 1-\mathrm{C} 4$ & $170.0(4)$ & $\mathrm{C} 12-\mathrm{C} 13-\mathrm{H} 13 \mathrm{C}$ & 109.5 \\
\hline
\end{tabular}




$$
\begin{aligned}
& \text { C7-Fe1-C5 } \\
& \text { C7-Fe1-C6 } \\
& \text { C7-Fe1-C9 } \\
& \text { C7-Fe1-C10 } \\
& \text { C8-Fe1-C1 } \\
& \text { C8-Fe1-C3 } \\
& \text { C8-Fe1-C4 } \\
& \text { C8-Fe1-C5 } \\
& \text { C8-Fe1-C6 } \\
& \text { C8-Fe1-C7 } \\
& \text { C8-Fe1-C9 } \\
& \text { C8-Fe1-C10 } \\
& \text { C9-Fe1-C5 } \\
& \text { C9-Fe1-C6 } \\
& \text { C9-Fe1-C10 } \\
& \text { C10-Fe1-C6 } \\
& \text { C15-Fe2-C21 } \\
& \text { C15-Fe2-C24 } \\
& \text { C16-Fe2-C15 } \\
& \text { C16-Fe2-C21 } \\
& \text { C16-Fe2-C24 } \\
& \text { C17-Fe2-C15 } \\
& \text { C17-Fe2-C16 } \\
& \text { C17-Fe2-C20 } \\
& \text { C17-Fe2-C21 } \\
& \text { C17-Fe2-C24 } \\
& \text { C18-Fe2-C15 } \\
& \text { C18-Fe2-C16 } \\
& \text { C18-Fe2-C17 } \\
& \text { C18-Fe2-C20 } \\
& \text { C18-Fe2-C21 } \\
& \text { C18-Fe2-C24 } \\
& \text { C19-Fe2-C15 } \\
& \text { C19-Fe2-C16 } \\
& \text { C19-Fe2-C17 } \\
& \text { C19-Fe2-C18 } \\
& \text { C19-Fe2-C20 } \\
& \text { C19-Fe2-C21 } \\
& \text { C19-Fe2-C22 } \\
& \text { C19-Fe2-C24 } \\
& \text { C20-Fe2-C15 } \\
& \text { C20-Fe2-C16 } \\
& \text { C20-Fe2-C21 } \\
& \text { C20-Fe2-C24 } \\
& \text { C22-Fe2-C15 } \\
& \text { C22-Fe2-C16 } \\
& \text { C22-Fe2-C17 } \\
& \text { C22-Fe2-C18 }
\end{aligned}
$$

\begin{tabular}{|c|c|}
\hline $\mathrm{H} 13 \mathrm{~A}-\mathrm{C} 13-\mathrm{H} 13 \mathrm{~B}$ & 109.5 \\
\hline $\mathrm{H} 13 \mathrm{~A}-\mathrm{C} 13-\mathrm{H} 13 \mathrm{C}$ & 109.5 \\
\hline $\mathrm{H} 13 \mathrm{~B}-\mathrm{C} 13-\mathrm{H} 13 \mathrm{C}$ & 109.5 \\
\hline $\mathrm{C} 11-\mathrm{C} 14-\mathrm{H} 14 \mathrm{~A}$ & 109.5 \\
\hline $\mathrm{C} 11-\mathrm{C} 14-\mathrm{H} 14 \mathrm{~B}$ & 109.5 \\
\hline $\mathrm{C} 11-\mathrm{C} 14-\mathrm{H} 14 \mathrm{C}$ & 109.5 \\
\hline $\mathrm{H} 14 \mathrm{~A}-\mathrm{C} 14-\mathrm{H} 14 \mathrm{~B}$ & 109.5 \\
\hline $\mathrm{H} 14 \mathrm{~A}-\mathrm{C} 14-\mathrm{H} 14 \mathrm{C}$ & 109.5 \\
\hline $\mathrm{H} 14 \mathrm{~B}-\mathrm{C} 14-\mathrm{H} 14 \mathrm{C}$ & 109.5 \\
\hline $\mathrm{P} 1-\mathrm{C} 15-\mathrm{Fe} 2$ & $129.5(4)$ \\
\hline $\mathrm{C} 16-\mathrm{C} 15-\mathrm{Fe} 2$ & $69.1(4)$ \\
\hline $\mathrm{C} 16-\mathrm{C} 15-\mathrm{P} 1$ & $123.9(6)$ \\
\hline $\mathrm{C} 19-\mathrm{C} 15-\mathrm{Fe} 2$ & $68.4(4)$ \\
\hline $\mathrm{C} 19-\mathrm{C} 15-\mathrm{P} 1$ & $129.0(6)$ \\
\hline $\mathrm{C} 19-\mathrm{C} 15-\mathrm{C} 16$ & $107.0(7)$ \\
\hline $\mathrm{C} 15-\mathrm{C} 16-\mathrm{Fe} 2$ & $69.6(4)$ \\
\hline $\mathrm{C} 15-\mathrm{C} 16-\mathrm{C} 25$ & $127.3(7)$ \\
\hline $\mathrm{C} 17-\mathrm{C} 16-\mathrm{Fe} 2$ & $69.3(5)$ \\
\hline $\mathrm{C} 17-\mathrm{C} 16-\mathrm{C} 15$ & $107.6(7)$ \\
\hline $\mathrm{C} 17-\mathrm{C} 16-\mathrm{C} 25$ & $125.0(7)$ \\
\hline $\mathrm{C} 25-\mathrm{C} 16-\mathrm{Fe} 2$ & $128.3(6)$ \\
\hline $\mathrm{Fe} 2-\mathrm{C} 17-\mathrm{H} 17$ & 126.8 \\
\hline $\mathrm{C} 16-\mathrm{C} 17-\mathrm{Fe} 2$ & $69.7(5)$ \\
\hline $\mathrm{C} 16-\mathrm{C} 17-\mathrm{H} 17$ & 125.7 \\
\hline $\mathrm{C} 18-\mathrm{C} 17-\mathrm{Fe} 2$ & $69.4(5)$ \\
\hline $\mathrm{C} 18-\mathrm{C} 17-\mathrm{C} 16$ & $108.6(7)$ \\
\hline $\mathrm{C} 18-\mathrm{C} 17-\mathrm{H} 17$ & 125.7 \\
\hline $\mathrm{Fe} 2-\mathrm{C} 18-\mathrm{H} 18$ & 126.5 \\
\hline $\mathrm{C} 17-\mathrm{C} 18-\mathrm{Fe} 2$ & $70.0(5)$ \\
\hline $\mathrm{C} 17-\mathrm{C} 18-\mathrm{H} 18$ & 126.0 \\
\hline $\mathrm{C} 17-\mathrm{C} 18-\mathrm{C} 19$ & $108.1(8)$ \\
\hline $\mathrm{C} 19-\mathrm{C} 18-\mathrm{Fe} 2$ & $69.1(5)$ \\
\hline $\mathrm{C} 19-\mathrm{C} 18-\mathrm{H} 18$ & 126.0 \\
\hline $\mathrm{Fe} 2-\mathrm{C} 19-\mathrm{H} 19$ & 125.7 \\
\hline $\mathrm{C} 15-\mathrm{C} 19-\mathrm{Fe} 2$ & $70.5(4)$ \\
\hline $\mathrm{C} 15-\mathrm{C} 19-\mathrm{H} 19$ & 125.7 \\
\hline $\mathrm{C} 18-\mathrm{C} 19-\mathrm{Fe} 2$ & $69.8(5)$ \\
\hline $\mathrm{C} 18-\mathrm{C} 19-\mathrm{C} 15$ & $108.7(7)$ \\
\hline $\mathrm{C} 18-\mathrm{C} 19-\mathrm{H} 19$ & 125.7 \\
\hline $\mathrm{Fe} 2-\mathrm{C} 20-\mathrm{H} 20$ & 125.5 \\
\hline $\mathrm{C} 21-\mathrm{C} 20-\mathrm{Fe} 2$ & $70.5(5)$ \\
\hline $\mathrm{C} 21-\mathrm{C} 20-\mathrm{H} 20$ & 125.0 \\
\hline $\mathrm{C} 24-\mathrm{C} 20-\mathrm{Fe} 2$ & $70.6(6)$ \\
\hline $\mathrm{C} 24-\mathrm{C} 20-\mathrm{H} 20$ & 125.0 \\
\hline $\mathrm{C} 24-\mathrm{C} 20-\mathrm{C} 21$ & $109.9(9)$ \\
\hline $\mathrm{Fe} 2-\mathrm{C} 21-\mathrm{H} 21$ & 127.0 \\
\hline $\mathrm{C} 20-\mathrm{C} 21-\mathrm{Fe} 2$ & $69.3(5)$ \\
\hline $\mathrm{C} 20-\mathrm{C} 21-\mathrm{H} 21$ & 126.7 \\
\hline
\end{tabular}

$$
\begin{aligned}
& 148.6(4) \\
& 41.2(4) \\
& 67.7(4) \\
& 67.8(4) \\
& 147.8(4) \\
& 108.6(4) \\
& 131.3(4) \\
& 169.9(4) \\
& 68.5(4) \\
& 40.3(4) \\
& 40.2(4) \\
& 67.6(4) \\
& 131.3(4) \\
& 67.8(4) \\
& 40.1(3) \\
& 40.0(4) \\
& 127.2(4) \\
& 124.7(3) \\
& 41.3(3) \\
& 111.6(4) \\
& 163.3(4) \\
& 69.1(3) \\
& 41.0(3) \\
& 163.3(4) \\
& 124.8(4) \\
& 153.6(4) \\
& 69.2(3) \\
& 69.0(3) \\
& 40.5(3) \\
& 156.1(4) \\
& 157.4(4) \\
& 118.4(4) \\
& 41.1(3) \\
& 69.2(3) \\
& 68.8(3) \\
& 41.1(3) \\
& 124.0(3) \\
& 161.5(4) \\
& 154.8(4) \\
& 105.8(3) \\
& 112.5(4) \\
& 129.1(4) \\
& 40.2(4) \\
& 39.8(4) \\
& 161.5(4) \\
& 123.1(4) \\
& 105.2(3) \\
& 118.5(4) \\
&
\end{aligned}
$$




\begin{tabular}{|c|c|c|c|}
\hline $\mathrm{C} 22-\mathrm{Fe} 2-\mathrm{C} 20$ & $67.9(4)$ & $\mathrm{C} 20-\mathrm{C} 21-\mathrm{C} 22$ & $106.6(9)$ \\
\hline $\mathrm{C} 22-\mathrm{Fe} 2-\mathrm{C} 21$ & 40.9 (4) & $\mathrm{C} 22-\mathrm{C} 21-\mathrm{Fe} 2$ & $68.5(6)$ \\
\hline $\mathrm{C} 22-\mathrm{Fe} 2-\mathrm{C} 24$ & $68.3(4)$ & $\mathrm{C} 22-\mathrm{C} 21-\mathrm{H} 21$ & 126.7 \\
\hline $\mathrm{C} 23-\mathrm{Fe} 2-\mathrm{C} 15$ & $157.7(4)$ & $\mathrm{Fe} 2-\mathrm{C} 22-\mathrm{H} 22$ & 125.4 \\
\hline $\mathrm{C} 23-\mathrm{Fe} 2-\mathrm{C} 16$ & $156.2(3)$ & $\mathrm{C} 21-\mathrm{C} 22-\mathrm{Fe} 2$ & $70.6(6)$ \\
\hline $\mathrm{C} 23-\mathrm{Fe} 2-\mathrm{C} 17$ & $118.1(4)$ & $\mathrm{C} 21-\mathrm{C} 22-\mathrm{H} 22$ & 126.3 \\
\hline $\mathrm{C} 23-\mathrm{Fe} 2-\mathrm{C} 18$ & $101.8(4)$ & $\mathrm{C} 23-\mathrm{C} 22-\mathrm{Fe} 2$ & $69.4(5)$ \\
\hline $\mathrm{C} 23-\mathrm{Fe} 2-\mathrm{C} 19$ & $119.0(4)$ & $\mathrm{C} 23-\mathrm{C} 22-\mathrm{C} 21$ & $107.4(9)$ \\
\hline $\mathrm{C} 23-\mathrm{Fe} 2-\mathrm{C} 20$ & $67.3(4)$ & $\mathrm{C} 23-\mathrm{C} 22-\mathrm{H} 22$ & 126.3 \\
\hline $\mathrm{C} 23-\mathrm{Fe} 2-\mathrm{C} 21$ & $68.2(4)$ & $\mathrm{Fe} 2-\mathrm{C} 23-\mathrm{H} 23$ & 125.1 \\
\hline $\mathrm{C} 23-\mathrm{Fe} 2-\mathrm{C} 22$ & 40.7 (4) & $\mathrm{C} 22-\mathrm{C} 23-\mathrm{Fe} 2$ & $69.9(5)$ \\
\hline $\mathrm{C} 23-\mathrm{Fe} 2-\mathrm{C} 24$ & $40.3(4)$ & $\mathrm{C} 22-\mathrm{C} 23-\mathrm{H} 23$ & 125.5 \\
\hline $\mathrm{C} 24-\mathrm{Fe} 2-\mathrm{C} 21$ & $67.9(4)$ & $\mathrm{C} 24-\mathrm{C} 23-\mathrm{Fe} 2$ & $71.1(5)$ \\
\hline $\mathrm{C} 1-\mathrm{P} 1-\mathrm{C} 15$ & $102.0(4)$ & $\mathrm{C} 24-\mathrm{C} 23-\mathrm{C} 22$ & $109.0(9)$ \\
\hline $\mathrm{C} 1-\mathrm{P} 1-\mathrm{C} 34$ & $100.1(4)$ & $\mathrm{C} 24-\mathrm{C} 23-\mathrm{H} 23$ & 125.5 \\
\hline $\mathrm{C} 15-\mathrm{P} 1-\mathrm{C} 34$ & $104.5(4)$ & $\mathrm{Fe} 2-\mathrm{C} 24-\mathrm{H} 24$ & 126.9 \\
\hline $\mathrm{C} 12-\mathrm{O} 1-\mathrm{C} 11$ & $118.1(7)$ & $\mathrm{C} 20-\mathrm{C} 24-\mathrm{Fe} 2$ & $69.6(5)$ \\
\hline $\mathrm{C} 26-\mathrm{O} 3-\mathrm{C} 25$ & $117.5(7)$ & $\mathrm{C} 20-\mathrm{C} 24-\mathrm{C} 23$ & $107.1(9)$ \\
\hline $\mathrm{P} 1-\mathrm{C} 1-\mathrm{Fe} 1$ & $125.7(4)$ & $\mathrm{C} 20-\mathrm{C} 24-\mathrm{H} 24$ & 126.5 \\
\hline $\mathrm{C} 2-\mathrm{C} 1-\mathrm{Fe} 1$ & $68.8(4)$ & $\mathrm{C} 23-\mathrm{C} 24-\mathrm{Fe} 2$ & $68.6(5)$ \\
\hline $\mathrm{C} 2-\mathrm{C} 1-\mathrm{P} 1$ & $123.6(6)$ & $\mathrm{C} 23-\mathrm{C} 24-\mathrm{H} 24$ & 126.5 \\
\hline $\mathrm{C} 2-\mathrm{C} 1-\mathrm{C} 5$ & $107.0(7)$ & $\mathrm{O} 3-\mathrm{C} 25-\mathrm{C} 16$ & $104.8(6)$ \\
\hline $\mathrm{C} 5-\mathrm{C} 1-\mathrm{Fe} 1$ & $69.7(5)$ & $\mathrm{O} 3-\mathrm{C} 25-\mathrm{H} 25$ & 110.2 \\
\hline $\mathrm{C} 5-\mathrm{C} 1-\mathrm{P} 1$ & $129.4(6)$ & $\mathrm{O} 3-\mathrm{C} 25-\mathrm{C} 28$ & $107.8(6)$ \\
\hline $\mathrm{C} 1-\mathrm{C} 2-\mathrm{Fe} 1$ & $69.7(5)$ & $\mathrm{C} 16-\mathrm{C} 25-\mathrm{H} 25$ & 110.2 \\
\hline $\mathrm{C} 1-\mathrm{C} 2-\mathrm{C} 11$ & $126.1(7)$ & $\mathrm{C} 16-\mathrm{C} 25-\mathrm{C} 28$ & $113.6(7)$ \\
\hline $\mathrm{C} 3-\mathrm{C} 2-\mathrm{Fe} 1$ & $70.4(5)$ & $\mathrm{C} 28-\mathrm{C} 25-\mathrm{H} 25$ & 110.2 \\
\hline $\mathrm{C} 3-\mathrm{C} 2-\mathrm{C} 1$ & $108.2(8)$ & $\mathrm{O} 3-\mathrm{C} 26-\mathrm{C} 27$ & $111.7(8)$ \\
\hline $\mathrm{C} 3-\mathrm{C} 2-\mathrm{C} 11$ & $125.4(8)$ & $\mathrm{O} 4-\mathrm{C} 26-\mathrm{O} 3$ & $124.2(8)$ \\
\hline $\mathrm{C} 11-\mathrm{C} 2-\mathrm{Fe} 1$ & $130.3(6)$ & $\mathrm{O} 4-\mathrm{C} 26-\mathrm{C} 27$ & $124.1(9)$ \\
\hline $\mathrm{Fe} 1-\mathrm{C} 3-\mathrm{H} 3$ & 127.6 & $\mathrm{C} 26-\mathrm{C} 27-\mathrm{H} 27 \mathrm{~A}$ & 109.5 \\
\hline $\mathrm{C} 2-\mathrm{C} 3-\mathrm{Fe} 1$ & $68.8(5)$ & $\mathrm{C} 26-\mathrm{C} 27-\mathrm{H} 27 \mathrm{~B}$ & 109.5 \\
\hline $\mathrm{C} 2-\mathrm{C} 3-\mathrm{H} 3$ & 126.0 & $\mathrm{C} 26-\mathrm{C} 27-\mathrm{H} 27 \mathrm{C}$ & 109.5 \\
\hline $\mathrm{C} 4-\mathrm{C} 3-\mathrm{Fe} 1$ & $69.2(5)$ & $\mathrm{H} 27 \mathrm{~A}-\mathrm{C} 27-\mathrm{H} 27 \mathrm{~B}$ & 109.5 \\
\hline $\mathrm{C} 4-\mathrm{C} 3-\mathrm{C} 2$ & $107.9(8)$ & $\mathrm{H} 27 \mathrm{~A}-\mathrm{C} 27-\mathrm{H} 27 \mathrm{C}$ & 109.5 \\
\hline $\mathrm{C} 4-\mathrm{C} 3-\mathrm{H} 3$ & 126.0 & $\mathrm{H} 27 \mathrm{~B}-\mathrm{C} 27-\mathrm{H} 27 \mathrm{C}$ & 109.5 \\
\hline $\mathrm{Fe} 1-\mathrm{C} 4-\mathrm{H} 4$ & 125.7 & $\mathrm{C} 25-\mathrm{C} 28-\mathrm{H} 28 \mathrm{~A}$ & 109.5 \\
\hline $\mathrm{C} 3-\mathrm{C} 4-\mathrm{Fe} 1$ & $70.2(5)$ & $\mathrm{C} 25-\mathrm{C} 28-\mathrm{H} 28 \mathrm{~B}$ & 109.5 \\
\hline $\mathrm{C} 3-\mathrm{C} 4-\mathrm{H} 4$ & 125.4 & $\mathrm{C} 25-\mathrm{C} 28-\mathrm{H} 28 \mathrm{C}$ & 109.5 \\
\hline $\mathrm{C} 5-\mathrm{C} 4-\mathrm{Fe} 1$ & $70.4(5)$ & $\mathrm{H} 28 \mathrm{~A}-\mathrm{C} 28-\mathrm{H} 28 \mathrm{~B}$ & 109.5 \\
\hline $\mathrm{C} 5-\mathrm{C} 4-\mathrm{C} 3$ & $109.2(8)$ & $\mathrm{H} 28 \mathrm{~A}-\mathrm{C} 28-\mathrm{H} 28 \mathrm{C}$ & 109.5 \\
\hline $\mathrm{C} 5-\mathrm{C} 4-\mathrm{H} 4$ & 125.4 & $\mathrm{H} 28 \mathrm{~B}-\mathrm{C} 28-\mathrm{H} 28 \mathrm{C}$ & 109.5 \\
\hline $\mathrm{Fe} 1-\mathrm{C} 5-\mathrm{H} 5$ & 127.5 & $\mathrm{C} 35-\mathrm{C} 34-\mathrm{P} 1$ & $115.6(6)$ \\
\hline $\mathrm{C} 1-\mathrm{C} 5-\mathrm{Fe} 1$ & $68.8(5)$ & $\mathrm{C} 39-\mathrm{C} 34-\mathrm{P} 1$ & $126.0(6)$ \\
\hline $\mathrm{C} 1-\mathrm{C} 5-\mathrm{H} 5$ & 126.2 & $\mathrm{C} 39-\mathrm{C} 34-\mathrm{C} 35$ & $118.4(8)$ \\
\hline $\mathrm{C} 4-\mathrm{C} 5-\mathrm{Fe} 1$ & $69.1(5)$ & $\mathrm{C} 34-\mathrm{C} 35-\mathrm{H} 35$ & 120.1 \\
\hline $\mathrm{C} 4-\mathrm{C} 5-\mathrm{C} 1$ & $107.6(8)$ & $\mathrm{C} 36-\mathrm{C} 35-\mathrm{C} 34$ & $119.9(8)$ \\
\hline $\mathrm{C} 4-\mathrm{C} 5-\mathrm{H} 5$ & 126.2 & $\mathrm{C} 36-\mathrm{C} 35-\mathrm{H} 35$ & 120.1 \\
\hline
\end{tabular}




\begin{tabular}{|c|c|c|c|}
\hline $\mathrm{Fe} 1-\mathrm{C} 6-\mathrm{H} 6$ & 126.2 & $\mathrm{C} 35-\mathrm{C} 36-\mathrm{H} 36$ & 119.6 \\
\hline $\mathrm{C} 7-\mathrm{C} 6-\mathrm{Fe} 1$ & $68.6(5)$ & $\mathrm{C} 37-\mathrm{C} 36-\mathrm{C} 35$ & $120.8(9)$ \\
\hline $\mathrm{C} 7-\mathrm{C} 6-\mathrm{H} 6$ & 126.7 & $\mathrm{C} 37-\mathrm{C} 36-\mathrm{H} 36$ & 119.6 \\
\hline $\mathrm{C} 10-\mathrm{C} 6-\mathrm{Fe} 1$ & $70.0(5)$ & $\mathrm{C} 36-\mathrm{C} 37-\mathrm{H} 37$ & 120.3 \\
\hline $\mathrm{C} 10-\mathrm{C} 6-\mathrm{H} 6$ & 126.7 & $\mathrm{C} 36-\mathrm{C} 37-\mathrm{C} 38$ & $119.4(9)$ \\
\hline $\mathrm{C} 10-\mathrm{C} 6-\mathrm{C} 7$ & $106.5(8)$ & $\mathrm{C} 38-\mathrm{C} 37-\mathrm{H} 37$ & 120.3 \\
\hline $\mathrm{Fe} 1-\mathrm{C} 7-\mathrm{H} 7$ & 125.7 & $\mathrm{C} 37-\mathrm{C} 38-\mathrm{H} 38$ & 119.9 \\
\hline $\mathrm{C} 6-\mathrm{C} 7-\mathrm{Fe} 1$ & $70.2(6)$ & $\mathrm{C} 39-\mathrm{C} 38-\mathrm{C} 37$ & $120.3(9)$ \\
\hline $\mathrm{C} 6-\mathrm{C} 7-\mathrm{H} 7$ & 125.9 & $\mathrm{C} 39-\mathrm{C} 38-\mathrm{H} 38$ & 119.9 \\
\hline $\mathrm{C} 8-\mathrm{C} 7-\mathrm{Fe} 1$ & $69.8(6)$ & $\mathrm{C} 34-\mathrm{C} 39-\mathrm{H} 39$ & 119.4 \\
\hline $\mathrm{C} 8-\mathrm{C} 7-\mathrm{C} 6$ & $108.1(10)$ & $\mathrm{C} 38-\mathrm{C} 39-\mathrm{C} 34$ & $121.2(8)$ \\
\hline $\mathrm{C} 8-\mathrm{C} 7-\mathrm{H} 7$ & 125.9 & $\mathrm{C} 38-\mathrm{C} 39-\mathrm{H} 39$ & 119.4 \\
\hline $\mathrm{Fe} 1-\mathrm{C} 8-\mathrm{H} 8$ & 125.3 & & \\
\hline $\mathrm{Fe} 1-\mathrm{C} 1-\mathrm{C} 2-\mathrm{C} 3$ & $60.0(6)$ & $\mathrm{C} 7-\mathrm{C} 6-\mathrm{C} 10-\mathrm{C} 9$ & $-0.1(10)$ \\
\hline $\mathrm{Fe} 1-\mathrm{C} 1-\mathrm{C} 2-\mathrm{C} 11$ & $-125.7(9)$ & $\mathrm{C} 7-\mathrm{C} 8-\mathrm{C} 9-\mathrm{Fe} 1$ & $59.9(6)$ \\
\hline $\mathrm{Fe} 1-\mathrm{C} 1-\mathrm{C} 5-\mathrm{C} 4$ & $-58.4(6)$ & $\mathrm{C} 7-\mathrm{C} 8-\mathrm{C} 9-\mathrm{C} 10$ & $0.4(10)$ \\
\hline $\mathrm{Fe} 1-\mathrm{C} 2-\mathrm{C} 3-\mathrm{C} 4$ & $58.2(6)$ & $\mathrm{C} 8-\mathrm{C} 9-\mathrm{C} 10-\mathrm{Fe} 1$ & $59.0(6)$ \\
\hline $\mathrm{Fe} 1-\mathrm{C} 2-\mathrm{C} 11-\mathrm{O} 1$ & $173.2(5)$ & $\mathrm{C} 8-\mathrm{C} 9-\mathrm{C} 10-\mathrm{C} 6$ & $-0.2(10)$ \\
\hline $\mathrm{Fe} 1-\mathrm{C} 2-\mathrm{C} 11-\mathrm{C} 14$ & $53.6(11)$ & $\mathrm{C} 10-\mathrm{C} 6-\mathrm{C} 7-\mathrm{Fe} 1$ & $60.1(6)$ \\
\hline $\mathrm{Fe} 1-\mathrm{C} 3-\mathrm{C} 4-\mathrm{C} 5$ & $59.7(6)$ & $\mathrm{C} 10-\mathrm{C} 6-\mathrm{C} 7-\mathrm{C} 8$ & $0.3(10)$ \\
\hline $\mathrm{Fe} 1-\mathrm{C} 4-\mathrm{C} 5-\mathrm{C} 1$ & $58.2(6)$ & $\mathrm{C} 11-\mathrm{O} 1-\mathrm{C} 12-\mathrm{O} 2$ & $-1.7(13)$ \\
\hline $\mathrm{Fe} 1-\mathrm{C} 6-\mathrm{C} 7-\mathrm{C} 8$ & $-59.7(6)$ & $\mathrm{C} 11-\mathrm{O} 1-\mathrm{C} 12-\mathrm{C} 13$ & $178.2(7)$ \\
\hline $\mathrm{Fe} 1-\mathrm{C} 6-\mathrm{C} 10-\mathrm{C} 9$ & $59.1(6)$ & $\mathrm{C} 11-\mathrm{C} 2-\mathrm{C} 3-\mathrm{Fe} 1$ & $126.1(9)$ \\
\hline $\mathrm{Fe} 1-\mathrm{C} 7-\mathrm{C} 8-\mathrm{C} 9$ & $-60.4(6)$ & $\mathrm{C} 11-\mathrm{C} 2-\mathrm{C} 3-\mathrm{C} 4$ & $-175.7(8)$ \\
\hline $\mathrm{Fe} 1-\mathrm{C} 8-\mathrm{C} 9-\mathrm{C} 10$ & $-59.5(6)$ & $\mathrm{C} 12-\mathrm{O} 1-\mathrm{C} 11-\mathrm{C} 2$ & $162.8(7)$ \\
\hline $\mathrm{Fe} 1-\mathrm{C} 9-\mathrm{C} 10-\mathrm{C} 6$ & $-59.2(6)$ & $\mathrm{C} 12-\mathrm{O} 1-\mathrm{C} 11-\mathrm{C} 14$ & $-74.0(9)$ \\
\hline $\mathrm{Fe} 2-\mathrm{C} 15-\mathrm{C} 16-\mathrm{C} 17$ & $59.1(6)$ & $\mathrm{C} 15-\mathrm{P} 1-\mathrm{C} 1-\mathrm{Fe} 1$ & $-98.9(5)$ \\
\hline $\mathrm{Fe} 2-\mathrm{C} 15-\mathrm{C} 16-\mathrm{C} 25$ & $-123.2(8)$ & $\mathrm{C} 15-\mathrm{P} 1-\mathrm{C} 1-\mathrm{C} 2$ & $174.5(7)$ \\
\hline $\mathrm{Fe} 2-\mathrm{C} 15-\mathrm{C} 19-\mathrm{C} 18$ & $-59.5(5)$ & $\mathrm{C} 15-\mathrm{P} 1-\mathrm{C} 1-\mathrm{C} 5$ & $-6.7(8)$ \\
\hline $\mathrm{Fe} 2-\mathrm{C} 16-\mathrm{C} 17-\mathrm{C} 18$ & $58.6(6)$ & $\mathrm{C} 15-\mathrm{P} 1-\mathrm{C} 34-\mathrm{C} 35$ & $122.9(6)$ \\
\hline $\mathrm{Fe} 2-\mathrm{C} 16-\mathrm{C} 25-\mathrm{O} 3$ & $173.3(5)$ & $\mathrm{C} 15-\mathrm{P} 1-\mathrm{C} 34-\mathrm{C} 39$ & $-55.1(8)$ \\
\hline $\mathrm{Fe} 2-\mathrm{C} 16-\mathrm{C} 25-\mathrm{C} 28$ & $55.9(10)$ & $\mathrm{C} 15-\mathrm{C} 16-\mathrm{C} 17-\mathrm{Fe} 2$ & $-59.3(5)$ \\
\hline $\mathrm{Fe} 2-\mathrm{C} 17-\mathrm{C} 18-\mathrm{C} 19$ & $58.8(6)$ & $\mathrm{C} 15-\mathrm{C} 16-\mathrm{C} 17-\mathrm{C} 18$ & $-0.6(9)$ \\
\hline $\mathrm{Fe} 2-\mathrm{C} 18-\mathrm{C} 19-\mathrm{C} 15$ & $60.0(5)$ & $\mathrm{C} 15-\mathrm{C} 16-\mathrm{C} 25-\mathrm{O} 3$ & $-94.2(9)$ \\
\hline $\mathrm{Fe} 2-\mathrm{C} 20-\mathrm{C} 21-\mathrm{C} 22$ & $-58.6(6)$ & $\mathrm{C} 15-\mathrm{C} 16-\mathrm{C} 25-\mathrm{C} 28$ & $148.4(8)$ \\
\hline $\mathrm{Fe} 2-\mathrm{C} 20-\mathrm{C} 24-\mathrm{C} 23$ & $58.6(6)$ & $\mathrm{C} 16-\mathrm{C} 15-\mathrm{C} 19-\mathrm{Fe} 2$ & $58.6(5)$ \\
\hline $\mathrm{Fe} 2-\mathrm{C} 21-\mathrm{C} 22-\mathrm{C} 23$ & $-59.8(6)$ & $\mathrm{C} 16-\mathrm{C} 15-\mathrm{C} 19-\mathrm{C} 18$ & $-1.0(9)$ \\
\hline $\mathrm{Fe} 2-\mathrm{C} 22-\mathrm{C} 23-\mathrm{C} 24$ & $-60.6(6)$ & $\mathrm{C} 16-\mathrm{C} 17-\mathrm{C} 18-\mathrm{Fe} 2$ & $-58.8(6)$ \\
\hline $\mathrm{Fe} 2-\mathrm{C} 23-\mathrm{C} 24-\mathrm{C} 20$ & $-59.2(6)$ & $\mathrm{C} 16-\mathrm{C} 17-\mathrm{C} 18-\mathrm{C} 19$ & $0.0(9)$ \\
\hline $\mathrm{P} 1-\mathrm{C} 1-\mathrm{C} 2-\mathrm{Fe} 1$ & $119.6(6)$ & $\mathrm{C} 17-\mathrm{C} 16-\mathrm{C} 25-\mathrm{O} 3$ & $83.1(9)$ \\
\hline $\mathrm{P} 1-\mathrm{C} 1-\mathrm{C} 2-\mathrm{C} 3$ & $179.6(6)$ & $\mathrm{C} 17-\mathrm{C} 16-\mathrm{C} 25-\mathrm{C} 28$ & $-34.3(12)$ \\
\hline $\mathrm{P} 1-\mathrm{C} 1-\mathrm{C} 2-\mathrm{C} 11$ & $-6.1(12)$ & $\mathrm{C} 17-\mathrm{C} 18-\mathrm{C} 19-\mathrm{Fe} 2$ & $-59.4(6)$ \\
\hline $\mathrm{P} 1-\mathrm{C} 1-\mathrm{C} 5-\mathrm{Fe} 1$ & $-120.1(7)$ & $\mathrm{C} 17-\mathrm{C} 18-\mathrm{C} 19-\mathrm{C} 15$ & $0.6(9)$ \\
\hline $\mathrm{P} 1-\mathrm{C} 1-\mathrm{C} 5-\mathrm{C} 4$ & $-178.5(6)$ & $\mathrm{C} 19-\mathrm{C} 15-\mathrm{C} 16-\mathrm{Fe} 2$ & $-58.1(5)$ \\
\hline $\mathrm{P} 1-\mathrm{C} 15-\mathrm{C} 16-\mathrm{Fe} 2$ & $124.3(6)$ & $\mathrm{C} 19-\mathrm{C} 15-\mathrm{C} 16-\mathrm{C} 17$ & $1.0(9)$ \\
\hline $\mathrm{P} 1-\mathrm{C} 15-\mathrm{C} 16-\mathrm{C} 17$ & $-176.5(6)$ & $\mathrm{C} 19-\mathrm{C} 15-\mathrm{C} 16-\mathrm{C} 25$ & $178.7(8)$ \\
\hline $\mathrm{P} 1-\mathrm{C} 15-\mathrm{C} 16-\mathrm{C} 25$ & $1.2(12)$ & $\mathrm{C} 20-\mathrm{C} 21-\mathrm{C} 22-\mathrm{Fe} 2$ & $59.2(6)$ \\
\hline
\end{tabular}




$\begin{array}{ll}\mathrm{P} 1-\mathrm{C} 15-\mathrm{C} 19-\mathrm{Fe} 2 & -124.1(7) \\ \mathrm{P} 1-\mathrm{C} 15-\mathrm{C} 19-\mathrm{C} 18 & 176.4(6) \\ \mathrm{P} 1-\mathrm{C} 34-\mathrm{C} 35-\mathrm{C} 36 & -176.3(7) \\ \mathrm{P} 1-\mathrm{C} 34-\mathrm{C} 39-\mathrm{C} 38 & 175.0(6) \\ \mathrm{C} 1-\mathrm{P} 1-\mathrm{C} 15-\mathrm{Fe} 2 & -140.8(5) \\ \mathrm{C} 1-\mathrm{P} 1-\mathrm{C} 15-\mathrm{C} 16 & 129.3(7) \\ \mathrm{C} 1-\mathrm{P} 1-\mathrm{C} 15-\mathrm{C} 19 & -47.6(8) \\ \mathrm{C} 1-\mathrm{P} 1-\mathrm{C} 34-\mathrm{C} 35 & -131.8(6) \\ \mathrm{C} 1-\mathrm{P} 1-\mathrm{C} 34-\mathrm{C} 39 & 50.2(8) \\ \mathrm{C} 1-\mathrm{C} 2-\mathrm{C} 3-\mathrm{Fe} 1 & -59.6(6) \\ \mathrm{C} 1-\mathrm{C} 2-\mathrm{C} 3-\mathrm{C} 4 & -1.4(9) \\ \mathrm{C} 1-\mathrm{C} 2-\mathrm{C} 11-\mathrm{O} 1 & -93.7(10) \\ \mathrm{C} 1-\mathrm{C} 2-\mathrm{C} 11-\mathrm{C} 14 & 146.7(8) \\ \mathrm{C} 2-\mathrm{C} 1-\mathrm{C} 5-\mathrm{Fe} 1 & 58.9(6) \\ \mathrm{C} 2-\mathrm{C} 1-\mathrm{C} 5-\mathrm{C} 4 & 0.5(9) \\ \mathrm{C} 2-\mathrm{C} 3-\mathrm{C} 4-\mathrm{Fe} 1 & -58.0(6) \\ \mathrm{C} 2-\mathrm{C} 3-\mathrm{C} 4-\mathrm{C} 5 & 1.7(10) \\ \mathrm{C} 3-\mathrm{C} 2-\mathrm{C} 11-\mathrm{O} 1 & 79.6(9) \\ \mathrm{C} 3-\mathrm{C} 2-\mathrm{C} 11-\mathrm{C} 14 & -40.0(12) \\ \mathrm{C} 3-\mathrm{C} 4-\mathrm{C} 5-\mathrm{Fe} 1 & -59.6(6) \\ \mathrm{C} 3-\mathrm{C} 4-\mathrm{C} 5-\mathrm{C} 1 & -1.4(10) \\ \mathrm{C} 5-\mathrm{C} 1-\mathrm{C} 2-\mathrm{Fe} 1 & -59.5(6) \\ \mathrm{C} 5-\mathrm{C} 1-\mathrm{C} 2-\mathrm{C} 3 & 0.5(9) \\ \mathrm{C} 5-\mathrm{C} 1-\mathrm{C} 2-\mathrm{C} 11 & 174.8(8) \\ \mathrm{C} 6-\mathrm{C} 7-\mathrm{C} 8-\mathrm{Fe} 1 & 59.9(6) \\ \mathrm{C} 6-\mathrm{C} 7-\mathrm{C} 8-\mathrm{C} 9 & -0.5(10) \\ \mathrm{C} 7-\mathrm{C} 6-\mathrm{C} 10-\mathrm{Fe} 1 & -59.2(6) \\ & \end{array}$

$\begin{array}{ll}\mathrm{C} 20-\mathrm{C} 21-\mathrm{C} 22-\mathrm{C} 23 & -0.7(10) \\ \mathrm{C} 21-\mathrm{C} 20-\mathrm{C} 24-\mathrm{Fe} 2 & -59.7(6) \\ \mathrm{C} 21-\mathrm{C} 20-\mathrm{C} 24-\mathrm{C} 23 & -1.1(10) \\ \mathrm{C} 21-\mathrm{C} 22-\mathrm{C} 23-\mathrm{Fe} 2 & 60.6(6) \\ \mathrm{C} 21-\mathrm{C} 22-\mathrm{C} 23-\mathrm{C} 24 & 0.0(11) \\ \mathrm{C} 22-\mathrm{C} 23-\mathrm{C} 24-\mathrm{Fe} 2 & 59.9(6) \\ \mathrm{C} 22-\mathrm{C} 23-\mathrm{C} 24-\mathrm{C} 20 & 0.6(10) \\ \mathrm{C} 24-\mathrm{C} 20-\mathrm{C} 21-\mathrm{Fe} 2 & 59.7(6) \\ \mathrm{C} 24-\mathrm{C} 20-\mathrm{C} 21-\mathrm{C} 22 & 1.1(10) \\ \mathrm{C} 25-\mathrm{O} 3-\mathrm{C} 26-\mathrm{O} 4 & -3.3(12) \\ \mathrm{C} 25-\mathrm{O} 3-\mathrm{C} 26-\mathrm{C} 27 & 177.2(7) \\ \mathrm{C} 25-\mathrm{C} 16-\mathrm{C} 17-\mathrm{Fe} 2 & 123.0(8) \\ \mathrm{C} 25-\mathrm{C} 16-\mathrm{C} 17-\mathrm{C} 18 & -178.4(7) \\ \mathrm{C} 26-\mathrm{O} 3-\mathrm{C} 25-\mathrm{C} 16 & 166.5(6) \\ \mathrm{C} 26-\mathrm{O} 3-\mathrm{C} 25-\mathrm{C} 28 & -72.2(9) \\ \mathrm{C} 34-\mathrm{P} 1-\mathrm{C} 1-\mathrm{Fe} 1 & 153.8(5) \\ \mathrm{C} 34-\mathrm{P} 1-\mathrm{C} 1-\mathrm{C} 2 & 67.1(7) \\ \mathrm{C} 34-\mathrm{P} 1-\mathrm{C} 1-\mathrm{C} 5 & -114.0(8) \\ \mathrm{C} 34-\mathrm{P} 1-\mathrm{C} 15-\mathrm{Fe} 2 & -36.8(6) \\ \mathrm{C} 34-\mathrm{P} 1-\mathrm{C} 15-\mathrm{C} 16 & -126.8(7) \\ \mathrm{C} 34-\mathrm{P} 1-\mathrm{C} 15-\mathrm{C} 19 & 56.3(8) \\ \mathrm{C} 34-\mathrm{C} 35-\mathrm{C} 36-\mathrm{C} 37 & 0.3(14) \\ \mathrm{C} 35-\mathrm{C} 34-\mathrm{C} 39-\mathrm{C} 38 & -2.9(12) \\ \mathrm{C} 35-\mathrm{C} 36-\mathrm{C} 37-\mathrm{C} 38 & -1.4(14) \\ \mathrm{C} 36-\mathrm{C} 37-\mathrm{C} 38-\mathrm{C} 39 & 0.4(14) \\ \mathrm{C} 37-\mathrm{C} 38-\mathrm{C} 39-\mathrm{C} 34 & 1.8(13) \\ \mathrm{C} 39-\mathrm{C} 34-\mathrm{C} 35-\mathrm{C} 36 & 1.9(12) \\ & \end{array}$

$\left\{\left(2 S_{\mathrm{p}}\right)-2-[(1 R)-1\right.$-(Acetyloxy)ethyl] ferrocen-1-yl\}[(2S $)$-2-ethenylferrocen-1-yl]phenyl-(S)-phosphane sulfide (8a)

Crystal data

$\left[\mathrm{Fe}_{2}\left(\mathrm{C}_{5} \mathrm{H}_{5}\right)_{2}\left(\mathrm{C}_{22} \mathrm{H}_{21} \mathrm{O}_{2} \mathrm{PS}\right)\right]$

$M_{r}=622.30$

Orthorhombic, $P 22_{1} 2_{1} 2_{1}$

$a=7.4923(3) \AA$

$b=12.0133(4) \AA$

$c=31.758(1) \AA$

$V=2858.45(17) \AA^{3}$

$Z=4$

$F(000)=1288$

\section{Data collection}

Bruker D8 Venture diffractometer

Radiation source: sealed xray tube, Incoatec IuS $\varphi$ and $\omega$ scans

Absorption correction: multi-scan

(SADABS; Bruker, 2012)

$T_{\min }=0.607, T_{\max }=0.746$

52739 measured reflections
$D_{\mathrm{x}}=1.446 \mathrm{Mg} \mathrm{m}^{-3}$

Mo $K \alpha$ radiation, $\lambda=0.71073 \AA$

Cell parameters from 9862 reflections

$\theta=2.6-29.6^{\circ}$

$\mu=1.17 \mathrm{~mm}^{-1}$

$T=100 \mathrm{~K}$

Plate, clear orange

$0.21 \times 0.14 \times 0.05 \mathrm{~mm}$

8330 independent reflections

7214 reflections with $I>2 \sigma(I)$

$R_{\text {int }}=0.062$

$\theta_{\text {max }}=30.0^{\circ}, \theta_{\text {min }}=2.6^{\circ}$

$h=-10 \rightarrow 10$

$k=-16 \rightarrow 15$

$l=-44 \rightarrow 40$ 


\author{
Refinement \\ Refinement on $F^{2}$ \\ Least-squares matrix: full \\ $R\left[F^{2}>2 \sigma\left(F^{2}\right)\right]=0.032$ \\ $w R\left(F^{2}\right)=0.062$ \\ $S=1.04$ \\ 8330 reflections \\ 345 parameters \\ 0 restraints \\ Primary atom site location: structure-invariant \\ direct methods
}

Hydrogen site location: inferred from neighbouring sites

$\mathrm{H}$-atom parameters constrained

$w=1 /\left[\sigma^{2}\left(F_{\mathrm{o}}^{2}\right)+(0.0231 P)^{2}+0.8513 P\right]$

where $P=\left(F_{\mathrm{o}}{ }^{2}+2 F_{\mathrm{c}}{ }^{2}\right) / 3$

$(\Delta / \sigma)_{\max }=0.001$

$\Delta \rho_{\max }=0.34 \mathrm{e}^{-3}$

$\Delta \rho_{\min }=-0.34$ e $\AA^{-3}$

Absolute structure: Flack $x$ determined using 2809 quotients $[(\mathrm{I}+)-(\mathrm{I}-)] /[(\mathrm{I}+)+(\mathrm{I}-)]$ (Parsons et al., 2013)

Absolute structure parameter: -0.007 (5)

Special details

Geometry. All esds (except the esd in the dihedral angle between two l.s. planes) are estimated using the full covariance matrix. The cell esds are taken into account individually in the estimation of esds in distances, angles and torsion angles; correlations between esds in cell parameters are only used when they are defined by crystal symmetry. An approximate (isotropic) treatment of cell esds is used for estimating esds involving l.s. planes.

Refinement._olex2_refinement_description 1. Fixed Uiso At 1.2 times of: All C(H) groups, $\mathrm{All} \mathrm{C}(\mathrm{H}, \mathrm{H})$ groups At 1.5 times of: $\mathrm{All} \mathrm{C}(\mathrm{H}, \mathrm{H}, \mathrm{H})$ groups 2 .a Ternary $\mathrm{CH}$ refined with riding coordinates: $\mathrm{C} 25(\mathrm{H} 25)$ 2.b Aromatic/amide $\mathrm{H}$ refined with riding coordinates: $\mathrm{C} 3(\mathrm{H} 3), \mathrm{C} 4(\mathrm{H} 4)$, C5(H5), C6(H6), C7(H7), C8(H8), C9(H9), C10(H10), C11(H11), C17(H17), C18(H18), C19(H19), C20(H20), C21(H21), C22(H22), C23(H23), C24(H24), C35(H35), C36(H36), C37(H37), C38(H38), C39(H39) 2.c X=CH2 refined with riding coordinates: $\mathrm{C} 12(\mathrm{H} 12 \mathrm{~A}, \mathrm{H} 12 \mathrm{~B})$ 2.d Idealised Me refined as rotating group: $\mathrm{C} 27(\mathrm{H} 27 \mathrm{~A}, \mathrm{H} 27 \mathrm{~B}, \mathrm{H} 27 \mathrm{C})$, C28(H28A,H28B,H28C)

Fractional atomic coordinates and isotropic or equivalent isotropic displacement parameters $\left(\AA^{2}\right)$

\begin{tabular}{lllll}
\hline & $x$ & $y$ & $z$ & $U_{\text {iso }} * U_{\text {eq }}$ \\
\hline Fe1 & $0.84121(5)$ & $-0.18090(3)$ & $0.40555(2)$ & $0.01684(9)$ \\
Fe2 & $0.60566(5)$ & $0.30603(3)$ & $0.41975(2)$ & $0.01652(9)$ \\
S1 & $0.45722(9)$ & $0.02593(6)$ & $0.34750(2)$ & $0.01925(14)$ \\
P1 & $0.70409(9)$ & $0.06730(5)$ & $0.35977(2)$ & $0.01195(13)$ \\
O1 & $0.6817(2)$ & $0.30513(15)$ & $0.28448(5)$ & $0.0175(4)$ \\
O2 & $0.4435(3)$ & $0.26315(16)$ & $0.24431(6)$ & $0.0236(4)$ \\
C1 & $0.8179(4)$ & $-0.01370(19)$ & $0.39926(7)$ & $0.0148(5)$ \\
C2 & $0.7513(4)$ & $-0.0495(2)$ & $0.43976(8)$ & $0.0183(6)$ \\
C3 & $0.8949(4)$ & $-0.1031(2)$ & $0.46113(8)$ & $0.0230(6)$ \\
H3 & 0.888873 & -0.135570 & 0.488372 & $0.028^{*}$ \\
C4 & $1.0480(4)$ & $-0.0999(2)$ & $0.43502(9)$ & $0.0254(6)$ \\
H4 & 1.161922 & -0.129537 & 0.441885 & $0.030^{*}$ \\
C5 & $1.0028(4)$ & $-0.0452(2)$ & $0.39690(8)$ & $0.0190(6)$ \\
H5 & 1.080914 & -0.031729 & 0.373894 & $0.023^{*}$ \\
C6 & $0.7115(5)$ & $-0.2603(2)$ & $0.35696(9)$ & $0.0329(8)$ \\
H6 & 0.659643 & -0.226079 & 0.332938 & $0.039^{*}$ \\
C7 & $0.6251(4)$ & $-0.2816(2)$ & $0.39548(9)$ & $0.0275(7)$ \\
H7 & 0.504649 & -0.264033 & 0.402018 & $0.033^{*}$ \\
C8 & $0.7482(4)$ & $-0.3334(2)$ & $0.42265(9)$ & $0.0265(6)$ \\
H8 & 0.724760 & -0.357321 & 0.450621 & $0.032^{*}$ \\
C9 & $0.9111(5)$ & $-0.3437(2)$ & $0.40135(10)$ & $0.0301(7)$
\end{tabular}




\begin{tabular}{|c|c|c|c|c|}
\hline H9 & 1.017630 & -0.375068 & 0.412418 & $0.036^{*}$ \\
\hline $\mathrm{C} 10$ & $0.8890(5)$ & $-0.2990(2)$ & $0.36035(9)$ & $0.0346(7)$ \\
\hline $\mathrm{H} 10$ & 0.977676 & -0.295624 & 0.339000 & $0.042 *$ \\
\hline C11 & $0.5680(4)$ & $-0.0391(2)$ & $0.45499(8)$ & $0.0246(6)$ \\
\hline H11 & 0.476791 & -0.027092 & 0.434716 & $0.030^{*}$ \\
\hline $\mathrm{C} 12$ & $0.5201(5)$ & $-0.0454(3)$ & $0.49504(9)$ & $0.0380(8)$ \\
\hline $\mathrm{H} 12 \mathrm{~A}$ & 0.607820 & -0.057404 & 0.516165 & $0.046^{*}$ \\
\hline H12B & 0.398118 & -0.037912 & 0.502653 & $0.046^{*}$ \\
\hline $\mathrm{C} 15$ & $0.7387(3)$ & $0.2077(2)$ & $0.37792(7)$ & $0.0133(5)$ \\
\hline $\mathrm{C} 16$ & $0.6821(3)$ & $0.3103(2)$ & $0.35835(7)$ & $0.0154(5)$ \\
\hline $\mathrm{C} 17$ & $0.7645(4)$ & $0.3989(2)$ & $0.38082(8)$ & 0.0183 \\
\hline H17 & 0.752333 & 0.475814 & 0.374486 & $0.022 *$ \\
\hline $\mathrm{C} 18$ & $0.8673(4)$ & $0.3543(2)$ & $0.41406(8)$ & $0.0191(5)$ \\
\hline H18 & 0.934574 & 0.395864 & 0.433926 & $0.023 *$ \\
\hline $\mathrm{C} 19$ & $0.8526(4)$ & $0.2363(2)$ & $0.41266(7)$ & 0.0168 \\
\hline H19 & 0.908377 & 0.185425 & 0.431394 & $0.020 *$ \\
\hline $\mathrm{C} 20$ & $0.3838(4)$ & $0.2247(2)$ & $0.44182(9)$ & 0.0270 \\
\hline $\mathrm{H} 20$ & 0.345910 & 0.151978 & 0.434147 & $0.032^{*}$ \\
\hline $\mathrm{C} 21$ & $0.3342(4)$ & $0.3246(2)$ & $0.42145(9)$ & $0.0294(6)$ \\
\hline $\mathrm{H} 21$ & 0.257300 & 0.330909 & 0.397791 & $0.035^{*}$ \\
\hline $\mathrm{C} 22$ & $0.4204(4)$ & $0.4145(3)$ & $0.44278(10)$ & $0.0312(7)$ \\
\hline $\mathrm{H} 22$ & 0.411066 & 0.491136 & 0.435758 & $0.037 *$ \\
\hline $\mathrm{C} 23$ & $0.5225(5)$ & $0.3691(3)$ & $0.47631(9)$ & $0.0306(7)$ \\
\hline $\mathrm{H} 23$ & 0.593323 & 0.409909 & 0.495730 & $0.037^{*}$ \\
\hline $\mathrm{C} 24$ & $0.4997(4)$ & $0.2512(3)$ & $0.47563(9)$ & $0.0291(7)$ \\
\hline $\mathrm{H} 24$ & 0.552832 & 0.199583 & 0.494519 & $0.035^{*}$ \\
\hline $\mathrm{C} 25$ & $0.5635(3)$ & $0.3234(2)$ & $0.32061(7)$ & $0.0166(5)$ \\
\hline $\mathrm{H} 25$ & 0.468303 & 0.265037 & 0.321063 & $0.020^{*}$ \\
\hline $\mathrm{C} 26$ & $0.6027(4)$ & $0.2739(2)$ & $0.24842(8)$ & $0.0193(6)$ \\
\hline $\mathrm{C} 27$ & $0.7367(4)$ & $0.2552(2)$ & $0.21417(8)$ & $0.0261(6)$ \\
\hline $\mathrm{H} 27 \mathrm{~A}$ & 0.785854 & 0.326950 & 0.205125 & $0.039^{*}$ \\
\hline H27B & 0.678720 & 0.218646 & 0.190224 & $0.039 *$ \\
\hline $\mathrm{H} 27 \mathrm{C}$ & 0.833351 & 0.207881 & 0.224748 & $0.039^{*}$ \\
\hline $\mathrm{C} 28$ & $0.4786(4)$ & $0.4381(2)$ & $0.31699(9)$ & $0.0250(6)$ \\
\hline $\mathrm{H} 28 \mathrm{~A}$ & 0.571985 & 0.495146 & 0.317601 & $0.037 *$ \\
\hline $\mathrm{H} 28 \mathrm{~B}$ & 0.396514 & 0.449686 & 0.340620 & $0.037 *$ \\
\hline $\mathrm{H} 28 \mathrm{C}$ & 0.412520 & 0.443228 & 0.290430 & $0.037 *$ \\
\hline C34 & $0.8497(3)$ & 0.05608 (19) & $0.31425(7)$ & $0.0142(5)$ \\
\hline $\mathrm{C} 35$ & $0.9988(4)$ & $0.1234(2)$ & $0.30989(8)$ & $0.0186(5)$ \\
\hline H35 & 1.024300 & 0.178211 & 0.330583 & $0.022 *$ \\
\hline $\mathrm{C} 36$ & $1.1110(4)$ & $0.1110(2)$ & $0.27541(9)$ & $0.0247(6)$ \\
\hline H36 & 1.211512 & 0.158452 & 0.272101 & $0.030 *$ \\
\hline C37 & $1.0754(4)$ & $0.0290(3)$ & $0.24583(8)$ & $0.0268(7)$ \\
\hline H37 & 1.152680 & 0.019673 & 0.222391 & $0.032 *$ \\
\hline C38 & $0.9290(4)$ & $-0.0387(2)$ & $0.25025(8)$ & $0.0265(7)$ \\
\hline H38 & 0.906227 & -0.095129 & 0.229993 & $0.032 *$ \\
\hline C39 & $0.8142(4)$ & $-0.0252(2)$ & $0.28415(8)$ & $0.0195(5)$ \\
\hline H39 & 0.711711 & -0.071215 & 0.286801 & $0.023 *$ \\
\hline
\end{tabular}


Atomic displacement parameters $\left(\AA^{2}\right)$

\begin{tabular}{|c|c|c|c|c|c|c|}
\hline & $U^{11}$ & $U^{22}$ & $U^{33}$ & $U^{12}$ & $U^{13}$ & $U^{23}$ \\
\hline Fe1 & $0.0231(2)$ & $0.01293(16)$ & $0.01450(16)$ & $-0.00341(15)$ & $-0.00318(14)$ & $0.00026(14)$ \\
\hline $\mathrm{Fe} 2$ & 0.01752 (19) & $0.01750(17)$ & $0.01454(16)$ & $-0.00029(15)$ & $0.00374(14)$ & $-0.00430(14)$ \\
\hline $\mathrm{S} 1$ & $0.0127(3)$ & $0.0242(3)$ & $0.0209(3)$ & $-0.0063(3)$ & $-0.0003(3)$ & $-0.0018(3)$ \\
\hline P1 & 0.0113 & $0.0130(3)$ & $0.0116(3)$ & $-0.0025(2)$ & $0.0007(2)$ & $-0.0012(2)$ \\
\hline $\mathrm{O} 1$ & $0.0184(9)$ & $0.0206(8)$ & $0.0136(8)$ & $-0.0016(8)$ & $0.0010(7)$ & $0.0006(7)$ \\
\hline $\mathrm{O} 2$ & $0.0214(11)$ & $0.0287(10)$ & $0.0207(9)$ & $-0.0047(9)$ & $-0.0054(8)$ & $0.0040(8)$ \\
\hline $\mathrm{C} 1$ & $0.0188(13)$ & $0.0124(10)$ & $0.0133(11)$ & $-0.0021(10)$ & $-0.0010(10)$ & $-0.0003(9)$ \\
\hline $\mathrm{C} 2$ & $0.0269(15)$ & $0.0146(12)$ & $0.0134(11)$ & $-0.0072(11)$ & $-0.0002(10)$ & $-0.0033(10)$ \\
\hline $\mathrm{C} 3$ & $0.0358(17)$ & $0.0188(12)$ & $0.0144(11)$ & $-0.0083(12)$ & $-0.0098(12)$ & $0.0003(10)$ \\
\hline $\mathrm{C} 4$ & $0.0277(16)$ & $0.0198(13)$ & $0.0287(14)$ & $-0.0040(12)$ & $-0.0142(13)$ & $0.0005(11)$ \\
\hline $\mathrm{C} 5$ & $0.0198(14)$ & $0.0150(12)$ & $0.0221(13)$ & $-0.0034(10)$ & $-0.0025(11)$ & $-0.0004(10)$ \\
\hline C6 & $0.057(2)$ & $0.0205(13)$ & $0.0216(14)$ & $-0.0149(14)$ & $-0.0163(15)$ & $0.0001(11)$ \\
\hline $\mathrm{C} 7$ & $0.0308(18)$ & $0.0168(12)$ & $0.0350(16)$ & $-0.0085(12)$ & $-0.0030(14)$ & $-0.0040(11)$ \\
\hline $\mathrm{C} 8$ & 0.0429 (19) & $0.0139(12)$ & $0.0226(13)$ & $-0.0077(12)$ & $-0.0001(13)$ & $0.0016(11)$ \\
\hline $\mathrm{C} 9$ & 0.0397 (19) & $0.0150(12)$ & $0.0357(15)$ & $0.0006(12)$ & $-0.0045(15)$ & $-0.0046(11)$ \\
\hline $\mathrm{C} 10$ & $0.049(2)$ & $0.0257(14)$ & $0.0292(15)$ & $-0.0113(15)$ & $0.0122(15)$ & $-0.0129(13)$ \\
\hline C11 & $0.0329(18)$ & $0.0222(14)$ & $0.0186(12)$ & $-0.0047(12)$ & $0.0068(12)$ & $-0.0007(10)$ \\
\hline $\mathrm{C} 12$ & $0.044(2)$ & $0.0470(19)$ & $0.0227(14)$ & $-0.0067(17)$ & $0.0117(14)$ & $0.0002(14)$ \\
\hline $\mathrm{C} 15$ & $0.0116(12)$ & $0.0145(11)$ & $0.0138(11)$ & $-0.0021(9)$ & $0.0023(9)$ & $-0.0031(9)$ \\
\hline $\mathrm{C} 16$ & $0.0139(12)$ & $0.0160(11)$ & $0.0164(11)$ & $-0.0021(11)$ & $0.0042(9)$ & $-0.0008(10)$ \\
\hline $\mathrm{C} 17$ & $0.0181(14)$ & $0.0157(12)$ & $0.0210(12)$ & $-0.0017(10)$ & $0.0046(11)$ & $-0.0031(10)$ \\
\hline $\mathrm{C} 18$ & $0.0167(14)$ & $0.0205(12)$ & $0.0200(12)$ & $-0.0029(10)$ & $-0.0013(11)$ & $-0.0072(10)$ \\
\hline C19 & $0.0178(14)$ & $0.0185(11)$ & $0.0142(11)$ & $-0.0008(10)$ & $0.0000(10)$ & $-0.0023(9)$ \\
\hline $\mathrm{C} 20$ & 0.0249 (16) & $0.0296(14)$ & 0.0265 (14) & $-0.0057(13)$ & $0.0144(13)$ & $-0.0052(11)$ \\
\hline $\mathrm{C} 21$ & 0.0189 (14) & $0.0390(16)$ & $0.0304(14)$ & 0.0033 (13) & $0.0090(12)$ & $-0.0015(14)$ \\
\hline $\mathrm{C} 22$ & $0.0305(18)$ & $0.0254(14)$ & $0.0379(16)$ & 0.0057 (13) & $0.0168(14)$ & $-0.0085(13)$ \\
\hline $\mathrm{C} 23$ & $0.0371(19)$ & $0.0316(15)$ & $0.0230(14)$ & 0.0005 (14) & $0.0134(13)$ & $-0.0124(12)$ \\
\hline $\mathrm{C} 24$ & $0.035(2)$ & $0.0334(16)$ & $0.0192(13)$ & -0.0010 & $0.0133(13)$ & $-0.0008(12)$ \\
\hline $\mathrm{C} 25$ & $0.0151(13)$ & $0.0180(12)$ & $0.0168(11)$ & $0.0013(10)$ & $0.0028(9)$ & $0.0001(10)$ \\
\hline $\mathrm{C} 26$ & $0.0243(16)$ & $0.0142(11)$ & $0.0195(12)$ & $0.0009(11)$ & $-0.0018(11)$ & $0.0043(9)$ \\
\hline $\mathrm{C} 27$ & $0.0283(17)$ & $0.0325(16)$ & $0.0176(13)$ & $0.0052(13)$ & $-0.0007(12)$ & $-0.0006(12)$ \\
\hline $\mathrm{C} 28$ & $0.0253(16)$ & $0.0253(14)$ & $0.0242(13)$ & $0.0065(12)$ & 0.0005 (12) & $0.0018(11)$ \\
\hline C34 & 0.0145 (13) & $0.0162(11)$ & $0.0119(10)$ & $0.0038(10)$ & $0.0001(9)$ & $0.0015(9)$ \\
\hline $\mathrm{C} 35$ & $0.0161(14)$ & $0.0204(13)$ & $0.0194(12)$ & $-0.0002(10)$ & $0.0010(10)$ & $-0.0015(10)$ \\
\hline $\mathrm{C} 36$ & $0.0164(14)$ & $0.0315(15)$ & $0.0261(14)$ & $0.0021(12)$ & $0.0066(12)$ & $0.0080(11)$ \\
\hline $\mathrm{C} 37$ & $0.0252(17)$ & $0.0359(16)$ & $0.0195(13)$ & $0.0146(14)$ & 0.0089 (11) & $0.0051(12)$ \\
\hline $\mathrm{C} 38$ & $0.0336(18)$ & $0.0271(15)$ & $0.0187(12)$ & $0.0080(13)$ & $0.0020(12)$ & $-0.0056(11)$ \\
\hline C39 & $0.0230(15)$ & $0.0182(12)$ & $0.0171(11)$ & $0.0001(11)$ & $-0.0005(11)$ & $-0.0002(10)$ \\
\hline
\end{tabular}

Geometric parameters $\left(A,{ }^{\circ}\right)$

\begin{tabular}{llll}
\hline $\mathrm{Fe} 1-\mathrm{C} 1$ & $2.026(2)$ & $\mathrm{C} 10-\mathrm{H} 10$ & 0.9500 \\
$\mathrm{Fe} 1-\mathrm{C} 2$ & $2.032(3)$ & $\mathrm{C} 11-\mathrm{H} 11$ & 0.9500 \\
$\mathrm{Fe} 1-\mathrm{C} 3$ & $2.038(2)$ & $\mathrm{C} 11-\mathrm{C} 12$ & $1.324(4)$ \\
$\mathrm{Fe} 1-\mathrm{C} 4$ & $2.055(3)$ & $\mathrm{C} 12-\mathrm{H} 12 \mathrm{~A}$ & 0.9500
\end{tabular}




\begin{tabular}{|c|c|c|c|}
\hline $\mathrm{Fe} 1-\mathrm{C} 5$ & $2.049(3)$ & $\mathrm{C} 12-\mathrm{H} 12 \mathrm{~B}$ & 0.9500 \\
\hline $\mathrm{Fe} 1-\mathrm{C} 6$ & $2.058(3)$ & $\mathrm{C} 15-\mathrm{C} 16$ & $1.444(3)$ \\
\hline $\mathrm{Fe} 1-\mathrm{C} 7$ & $2.046(3)$ & $\mathrm{C} 15-\mathrm{C} 19$ & $1.437(3)$ \\
\hline $\mathrm{Fe} 1-\mathrm{C} 8$ & $2.034(3)$ & $\mathrm{C} 16-\mathrm{C} 17$ & $1.423(4)$ \\
\hline $\mathrm{Fe} 1-\mathrm{C} 9$ & $2.029(3)$ & $\mathrm{C} 16-\mathrm{C} 25$ & $1.500(3)$ \\
\hline $\mathrm{Fe} 1-\mathrm{C} 10$ & $2.050(3)$ & C17-H17 & 0.9500 \\
\hline $\mathrm{Fe} 2-\mathrm{C} 15$ & $2.038(2)$ & $\mathrm{C} 17-\mathrm{C} 18$ & $1.412(4)$ \\
\hline $\mathrm{Fe} 2-\mathrm{C} 16$ & $2.033(2)$ & C18-H18 & 0.9500 \\
\hline $\mathrm{Fe} 2-\mathrm{C} 17$ & $2.047(3)$ & $\mathrm{C} 18-\mathrm{C} 19$ & $1.422(3)$ \\
\hline $\mathrm{Fe} 2-\mathrm{C} 18$ & $2.052(3)$ & C19-H19 & 0.9500 \\
\hline $\mathrm{Fe} 2-\mathrm{C} 19$ & $2.043(3)$ & $\mathrm{C} 20-\mathrm{H} 20$ & 0.9500 \\
\hline $\mathrm{Fe} 2-\mathrm{C} 20$ & $2.052(3)$ & $\mathrm{C} 20-\mathrm{C} 21$ & $1.414(4)$ \\
\hline $\mathrm{Fe} 2-\mathrm{C} 21$ & $2.047(3)$ & $\mathrm{C} 20-\mathrm{C} 24$ & $1.417(4)$ \\
\hline $\mathrm{Fe} 2-\mathrm{C} 22$ & $2.039(3)$ & $\mathrm{C} 21-\mathrm{H} 21$ & 0.9500 \\
\hline $\mathrm{Fe} 2-\mathrm{C} 23$ & $2.047(3)$ & $\mathrm{C} 21-\mathrm{C} 22$ & $1.429(4)$ \\
\hline $\mathrm{Fe} 2-\mathrm{C} 24$ & $2.053(3)$ & $\mathrm{C} 22-\mathrm{H} 22$ & 0.9500 \\
\hline $\mathrm{S} 1-\mathrm{P} 1$ & $1.9544(9)$ & $\mathrm{C} 22-\mathrm{C} 23$ & $1.420(5)$ \\
\hline $\mathrm{P} 1-\mathrm{C} 1$ & $1.802(2)$ & $\mathrm{C} 23-\mathrm{H} 23$ & 0.9500 \\
\hline $\mathrm{P} 1-\mathrm{C} 15$ & $1.801(2)$ & $\mathrm{C} 23-\mathrm{C} 24$ & $1.427(4)$ \\
\hline $\mathrm{P} 1-\mathrm{C} 34$ & $1.816(2)$ & $\mathrm{C} 24-\mathrm{H} 24$ & 0.9500 \\
\hline $\mathrm{O} 1-\mathrm{C} 25$ & $1.466(3)$ & $\mathrm{C} 25-\mathrm{H} 25$ & 1.0000 \\
\hline $\mathrm{O} 1-\mathrm{C} 26$ & $1.343(3)$ & $\mathrm{C} 25-\mathrm{C} 28$ & $1.522(4)$ \\
\hline $\mathrm{O} 2-\mathrm{C} 26$ & $1.207(3)$ & $\mathrm{C} 26-\mathrm{C} 27$ & $1.497(4)$ \\
\hline $\mathrm{C} 1-\mathrm{C} 2$ & $1.445(3)$ & $\mathrm{C} 27-\mathrm{H} 27 \mathrm{~A}$ & 0.9800 \\
\hline $\mathrm{C} 1-\mathrm{C} 5$ & $1.438(4)$ & C27-H27B & 0.9800 \\
\hline $\mathrm{C} 2-\mathrm{C} 3$ & $1.426(4)$ & $\mathrm{C} 27-\mathrm{H} 27 \mathrm{C}$ & 0.9800 \\
\hline $\mathrm{C} 2-\mathrm{C} 11$ & $1.461(4)$ & $\mathrm{C} 28-\mathrm{H} 28 \mathrm{~A}$ & 0.9800 \\
\hline $\mathrm{C} 3-\mathrm{H} 3$ & 0.9500 & $\mathrm{C} 28-\mathrm{H} 28 \mathrm{~B}$ & 0.9800 \\
\hline $\mathrm{C} 3-\mathrm{C} 4$ & $1.416(4)$ & $\mathrm{C} 28-\mathrm{H} 28 \mathrm{C}$ & 0.9800 \\
\hline $\mathrm{C} 4-\mathrm{H} 4$ & 0.9500 & $\mathrm{C} 34-\mathrm{C} 35$ & $1.386(4)$ \\
\hline $\mathrm{C} 4-\mathrm{C} 5$ & $1.419(4)$ & C34-C39 & $1.392(3)$ \\
\hline $\mathrm{C} 5-\mathrm{H} 5$ & 0.9500 & C $35-\mathrm{H} 35$ & 0.9500 \\
\hline $\mathrm{C} 6-\mathrm{H} 6$ & 0.9500 & $\mathrm{C} 35-\mathrm{C} 36$ & $1.388(4)$ \\
\hline $\mathrm{C} 6-\mathrm{C} 7$ & $1.407(4)$ & $\mathrm{C} 36-\mathrm{H} 36$ & 0.9500 \\
\hline $\mathrm{C} 6-\mathrm{C} 10$ & $1.413(5)$ & $\mathrm{C} 36-\mathrm{C} 37$ & $1.387(4)$ \\
\hline $\mathrm{C} 7-\mathrm{H} 7$ & 0.9500 & $\mathrm{C} 37-\mathrm{H} 37$ & 0.9500 \\
\hline $\mathrm{C} 7-\mathrm{C} 8$ & $1.408(4)$ & $\mathrm{C} 37-\mathrm{C} 38$ & $1.372(4)$ \\
\hline $\mathrm{C} 8-\mathrm{H} 8$ & 0.9500 & C $38-\mathrm{H} 38$ & 0.9500 \\
\hline $\mathrm{C} 8-\mathrm{C} 9$ & $1.401(4)$ & C38-C39 & $1.388(4)$ \\
\hline $\mathrm{C} 9-\mathrm{H} 9$ & 0.9500 & C39-H39 & 0.9500 \\
\hline $\mathrm{C} 9-\mathrm{C} 10$ & $1.418(4)$ & & \\
\hline $\mathrm{C} 1-\mathrm{Fe} 1-\mathrm{C} 2$ & $41.73(10)$ & $\mathrm{C} 10-\mathrm{C} 6-\mathrm{Fe} 1$ & $69.56(17)$ \\
\hline $\mathrm{C} 1-\mathrm{Fe} 1-\mathrm{C} 3$ & $69.38(10)$ & $\mathrm{C} 10-\mathrm{C} 6-\mathrm{H} 6$ & 126.1 \\
\hline $\mathrm{C} 1-\mathrm{Fe} 1-\mathrm{C} 4$ & $68.95(11)$ & $\mathrm{Fe} 1-\mathrm{C} 7-\mathrm{H} 7$ & 125.9 \\
\hline $\mathrm{C} 1-\mathrm{Fe} 1-\mathrm{C} 5$ & $41.33(11)$ & $\mathrm{C} 6-\mathrm{C} 7-\mathrm{Fe} 1$ & $70.41(17)$ \\
\hline $\mathrm{C} 1-\mathrm{Fe} 1-\mathrm{C} 6$ & $110.18(11)$ & $\mathrm{C} 6-\mathrm{C} 7-\mathrm{H} 7$ & 125.9 \\
\hline $\mathrm{C} 1-\mathrm{Fe} 1-\mathrm{C} 7$ & $120.16(11)$ & $\mathrm{C} 6-\mathrm{C} 7-\mathrm{C} 8$ & $108.2(3)$ \\
\hline
\end{tabular}




$$
\begin{aligned}
& \text { C1-Fe1-C8 } \\
& \text { C1-Fe1-C9 } \\
& \text { C1-Fe1-C10 } \\
& \text { C2-Fe1-C3 } \\
& \text { C2-Fe1-C4 } \\
& \text { C2-Fe1-C5 } \\
& \text { C2-Fe1-C6 } \\
& \text { C2-Fe1-C7 } \\
& \text { C2-Fe1-C8 } \\
& \text { C2-Fe1-C10 } \\
& \text { C3-Fe1-C4 } \\
& \text { C3-Fe1-C5 } \\
& \text { C3-Fe1-C6 } \\
& \text { C3-Fe1-C7 } \\
& \text { C3-Fe1-C10 } \\
& \text { C4-Fe1-C6 } \\
& \text { C5-Fe1-C4 } \\
& \text { C5-Fe1-C6 } \\
& \text { C5-Fe1-C10 } \\
& \text { C7-Fe1-C4 } \\
& \text { C7-Fe1-C5 } \\
& \text { C7-Fe1-C6 } \\
& \text { C7-Fe1-C10 } \\
& \text { C8-Fe1-C3 } \\
& \text { C8-Fe1-C4 } \\
& \text { C8-Fe1-C5 } \\
& \text { C8-Fe1-C6 } \\
& \text { C8-Fe1-C7 } \\
& \text { C8-Fe1-C10 } \\
& \text { C9-Fe1-C2 } \\
& \text { C9-Fe1-C3 } \\
& \text { C9-Fe1-C4 } \\
& \text { C9-Fe1-C5 } \\
& \text { C9-Fe1-C6 } \\
& \text { C9-Fe1-C7 } \\
& \text { C9-Fe1-C8 } \\
& \text { C9-Fe1-C10 } \\
& \text { C10-Fe1-C4 } \\
& \text { C10-Fe1-C6 } \\
& \text { C15-Fe2-C17 } \\
& \text { C15-Fe2-C18 } \\
& \text { C15-Fe2-C19 } \\
& \text { C15-Fe2-C20 } \\
& \text { C15-Fe2-C21 } \\
& \text { C15-Fe2-C22 } \\
& \text { C15-Fe2-C23 } \\
& \text { C15-Fe2-C24 } \\
& \text { C16-Fe2-C15 }
\end{aligned}
$$

$152.84(12)$

\begin{tabular}{|c|c|}
\hline $\mathrm{C} 8-\mathrm{C} 7-\mathrm{Fe} 1$ & $69.36(17)$ \\
\hline $\mathrm{C} 8-\mathrm{C} 7-\mathrm{H} 7$ & 125.9 \\
\hline $\mathrm{Fe} 1-\mathrm{C} 8-\mathrm{H} 8$ & 125.8 \\
\hline $\mathrm{C} 7-\mathrm{C} 8-\mathrm{Fe} 1$ & $70.28(15)$ \\
\hline $\mathrm{C} 7-\mathrm{C} 8-\mathrm{H} 8$ & 125.9 \\
\hline $\mathrm{C} 9-\mathrm{C} 8-\mathrm{Fe} 1$ & $69.63(15)$ \\
\hline $\mathrm{C} 9-\mathrm{C} 8-\mathrm{C} 7$ & $108.3(3)$ \\
\hline $\mathrm{C} 9-\mathrm{C} 8-\mathrm{H} 8$ & 125.9 \\
\hline $\mathrm{Fe} 1-\mathrm{C} 9-\mathrm{H} 9$ & 125.1 \\
\hline $\mathrm{C} 8-\mathrm{C} 9-\mathrm{Fe} 1$ & $70.03(16)$ \\
\hline $\mathrm{C} 8-\mathrm{C} 9-\mathrm{H} 9$ & 126.0 \\
\hline $\mathrm{C} 8-\mathrm{C} 9-\mathrm{C} 10$ & $108.0(3)$ \\
\hline $\mathrm{C} 10-\mathrm{C} 9-\mathrm{Fe} 1$ & $70.44(16)$ \\
\hline $\mathrm{C} 10-\mathrm{C} 9-\mathrm{H} 9$ & 126.0 \\
\hline $\mathrm{Fe} 1-\mathrm{C} 10-\mathrm{H} 10$ & 126.3 \\
\hline $\mathrm{C} 6-\mathrm{C} 10-\mathrm{Fe} 1$ & $70.21(17)$ \\
\hline $\mathrm{C} 6-\mathrm{C} 10-\mathrm{C} 9$ & $107.7(3)$ \\
\hline $\mathrm{C} 6-\mathrm{C} 10-\mathrm{H} 10$ & 126.1 \\
\hline $\mathrm{C} 9-\mathrm{C} 10-\mathrm{Fe} 1$ & $68.87(15)$ \\
\hline $\mathrm{C} 9-\mathrm{C} 10-\mathrm{H} 10$ & 126.1 \\
\hline $\mathrm{C} 2-\mathrm{C} 11-\mathrm{H} 11$ & 117.7 \\
\hline $\mathrm{C} 12-\mathrm{C} 11-\mathrm{C} 2$ & $124.6(3)$ \\
\hline $\mathrm{C} 12-\mathrm{C} 11-\mathrm{H} 11$ & 117.7 \\
\hline $\mathrm{C} 11-\mathrm{C} 12-\mathrm{H} 12 \mathrm{~A}$ & 120.0 \\
\hline $\mathrm{C} 11-\mathrm{C} 12-\mathrm{H} 12 \mathrm{~B}$ & 120.0 \\
\hline $\mathrm{H} 12 \mathrm{~A}-\mathrm{C} 12-\mathrm{H} 12 \mathrm{~B}$ & 120.0 \\
\hline $\mathrm{P} 1-\mathrm{C} 15-\mathrm{Fe} 2$ & $132.94(14)$ \\
\hline $\mathrm{C} 16-\mathrm{C} 15-\mathrm{Fe} 2$ & $69.03(13)$ \\
\hline $\mathrm{C} 16-\mathrm{C} 15-\mathrm{P} 1$ & $128.28(18)$ \\
\hline $\mathrm{C} 19-\mathrm{C} 15-\mathrm{Fe} 2$ & $69.58(14)$ \\
\hline $\mathrm{C} 19-\mathrm{C} 15-\mathrm{P} 1$ & $123.75(19)$ \\
\hline $\mathrm{C} 19-\mathrm{C} 15-\mathrm{C} 16$ & $107.5(2)$ \\
\hline $\mathrm{C} 15-\mathrm{C} 16-\mathrm{Fe} 2$ & $69.43(13)$ \\
\hline $\mathrm{C} 15-\mathrm{C} 16-\mathrm{C} 25$ & $127.4(2)$ \\
\hline $\mathrm{C} 17-\mathrm{C} 16-\mathrm{Fe} 2$ & $70.12(14)$ \\
\hline $\mathrm{C} 17-\mathrm{C} 16-\mathrm{C} 15$ & $107.2(2)$ \\
\hline $\mathrm{C} 17-\mathrm{C} 16-\mathrm{C} 25$ & $125.4(2)$ \\
\hline $\mathrm{C} 25-\mathrm{C} 16-\mathrm{Fe} 2$ & $127.01(17)$ \\
\hline $\mathrm{Fe} 2-\mathrm{C} 17-\mathrm{H} 17$ & 127.0 \\
\hline $\mathrm{C} 16-\mathrm{C} 17-\mathrm{Fe} 2$ & $69.06(14)$ \\
\hline $\mathrm{C} 16-\mathrm{C} 17-\mathrm{H} 17$ & 125.5 \\
\hline $\mathrm{C} 18-\mathrm{C} 17-\mathrm{Fe} 2$ & $70.03(16)$ \\
\hline $\mathrm{C} 18-\mathrm{C} 17-\mathrm{C} 16$ & $109.1(2)$ \\
\hline $\mathrm{C} 18-\mathrm{C} 17-\mathrm{H} 17$ & 125.5 \\
\hline $\mathrm{Fe} 2-\mathrm{C} 18-\mathrm{H} 18$ & 126.7 \\
\hline $\mathrm{C} 17-\mathrm{C} 18-\mathrm{Fe} 2$ & $69.66(16)$ \\
\hline $\mathrm{C} 17-\mathrm{C} 18-\mathrm{H} 18$ & 125.9 \\
\hline $\mathrm{C} 17-\mathrm{C} 18-\mathrm{C} 19$ & $108.2(2)$ \\
\hline
\end{tabular}

$166.25(12)$

$129.21(11)$

$41.03(11)$

$68.80(12)$

$69.47(11)$

$127.22(13)$

$106.31(12)$

$116.34(11)$

$166.03(12)$

$40.48(12)$

$68.56(11)$

$162.84(14)$

$124.25(12)$

$152.83(13)$

$156.49(13)$

$40.45(10)$

$123.19(11)$

$110.70(12)$

$161.36(11)$

$156.48(11)$

$40.10(12)$

$67.65(13)$

$104.45(11)$

$124.27(11)$

$162.71(12)$

$67.71(12)$

$40.36(12)$

$67.88(12)$

$150.44(11)$

$116.62(12)$

$106.95(13)$

$127.26(13)$

$68.02(13)$

$67.92(13)$

$40.34(13)$

40.69 (12)

120.98 (14)

40.23 (14)

$68.78(10)$

68.85 (10)

41.22 (9)

110.03 (10)

124.49 (11)

$159.53(12)$

159.31 (12)

124.51 (11)

41.54 (10) 


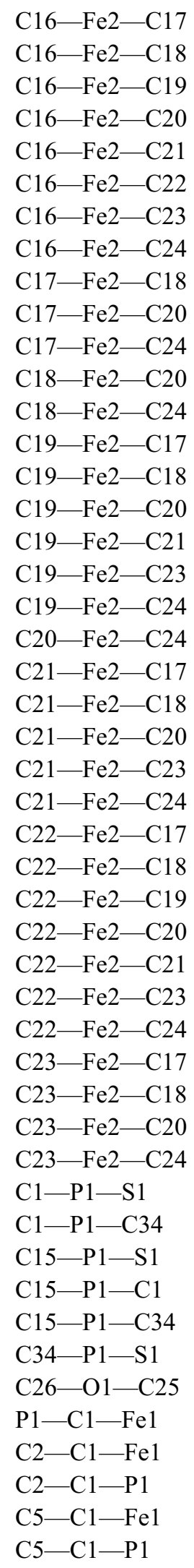

\begin{tabular}{|c|c|c|}
\hline $40.82(10)$ & $\mathrm{C} 19-\mathrm{C} 18-\mathrm{Fe} 2$ & $69.37(16)$ \\
\hline $68.85(10)$ & $\mathrm{C} 19-\mathrm{C} 18-\mathrm{H} 18$ & 125.9 \\
\hline $69.47(10)$ & $\mathrm{Fe} 2-\mathrm{C} 19-\mathrm{H} 19$ & 126.4 \\
\hline $124.60(11)$ & $\mathrm{C} 15-\mathrm{C} 19-\mathrm{Fe} 2$ & $69.19(15)$ \\
\hline $107.61(11)$ & $\mathrm{C} 15-\mathrm{C} 19-\mathrm{H} 19$ & 126.0 \\
\hline $121.31(12)$ & $\mathrm{C} 18-\mathrm{C} 19-\mathrm{Fe} 2$ & $70.00(16)$ \\
\hline $156.69(11)$ & $\mathrm{C} 18-\mathrm{C} 19-\mathrm{C} 15$ & $108.0(2)$ \\
\hline $161.13(11)$ & $\mathrm{C} 18-\mathrm{C} 19-\mathrm{H} 19$ & 126.0 \\
\hline $40.31(10)$ & $\mathrm{Fe} 2-\mathrm{C} 20-\mathrm{H} 20$ & 126.3 \\
\hline $159.57(12)$ & $\mathrm{C} 21-\mathrm{C} 20-\mathrm{Fe} 2$ & $69.63(16)$ \\
\hline $157.21(12)$ & $\mathrm{C} 21-\mathrm{C} 20-\mathrm{H} 20$ & 125.8 \\
\hline $159.80(12)$ & $\mathrm{C} 21-\mathrm{C} 20-\mathrm{C} 24$ & 108.5 \\
\hline $122.43(12)$ & $\mathrm{C} 24-\mathrm{C} 20-\mathrm{Fe} 2$ & $69.85(17)$ \\
\hline $68.30(10)$ & $\mathrm{C} 24-\mathrm{C} 20-\mathrm{H} 20$ & 125.8 \\
\hline $40.63(10)$ & $\mathrm{Fe} 2-\mathrm{C} 21-\mathrm{H} 21$ & 126.2 \\
\hline $125.16(11)$ & $\mathrm{C} 20-\mathrm{C} 21-\mathrm{Fe} 2$ & $70.01(17)$ \\
\hline $161.33(11)$ & $\mathrm{C} 20-\mathrm{C} 21-\mathrm{H} 21$ & 126.1 \\
\hline $121.61(12)$ & $\mathrm{C} 20-\mathrm{C} 21-\mathrm{C} 22$ & $107.8(3)$ \\
\hline $108.30(12)$ & $\mathrm{C} 22-\mathrm{C} 21-\mathrm{Fe} 2$ & $69.26(17)$ \\
\hline $40.40(12)$ & $\mathrm{C} 22-\mathrm{C} 21-\mathrm{H} 21$ & 126.1 \\
\hline $122.29(12)$ & $\mathrm{Fe} 2-\mathrm{C} 22-\mathrm{H} 22$ & 125.8 \\
\hline $157.03(11)$ & $\mathrm{C} 21-\mathrm{C} 22-\mathrm{Fe} 2$ & $69.81(16)$ \\
\hline $40.36(12)$ & $\mathrm{C} 21-\mathrm{C} 22-\mathrm{H} 22$ & 126.0 \\
\hline 68.53 (13) & $\mathrm{C} 23-\mathrm{C} 22-\mathrm{Fe} 2$ & $69.94(17)$ \\
\hline $68.16(13)$ & $\mathrm{C} 23-\mathrm{C} 22-\mathrm{C} 21$ & $108.0(3)$ \\
\hline $105.31(12)$ & $\mathrm{C} 23-\mathrm{C} 22-\mathrm{H} 22$ & 126.0 \\
\hline 120.09 (12) & $\mathrm{Fe} 2-\mathrm{C} 23-\mathrm{H} 23$ & 126.2 \\
\hline $156.58(12)$ & $\mathrm{C} 22-\mathrm{C} 23-\mathrm{Fe} 2$ & $69.38(16)$ \\
\hline $68.32(12)$ & $\mathrm{C} 22-\mathrm{C} 23-\mathrm{H} 23$ & 126.1 \\
\hline 40.93 (12) & $\mathrm{C} 22-\mathrm{C} 23-\mathrm{C} 24$ & $107.8(3)$ \\
\hline 40.68 (13) & $\mathrm{C} 24-\mathrm{C} 23-\mathrm{Fe} 2$ & $69.87(16)$ \\
\hline $68.40(13)$ & $\mathrm{C} 24-\mathrm{C} 23-\mathrm{H} 23$ & 126.1 \\
\hline $120.36(12)$ & $\mathrm{Fe} 2-\mathrm{C} 24-\mathrm{H} 24$ & 126.4 \\
\hline $105.29(12)$ & $\mathrm{C} 20-\mathrm{C} 24-\mathrm{Fe} 2$ & $69.76(16)$ \\
\hline $68.27(12)$ & $\mathrm{C} 20-\mathrm{C} 24-\mathrm{C} 23$ & $107.9(3)$ \\
\hline 40.73 (12) & $\mathrm{C} 20-\mathrm{C} 24-\mathrm{H} 24$ & 126.0 \\
\hline $116.69(9)$ & $\mathrm{C} 23-\mathrm{C} 24-\mathrm{Fe} 2$ & $69.40(17)$ \\
\hline $103.27(11)$ & $\mathrm{C} 23-\mathrm{C} 24-\mathrm{H} 24$ & 126.0 \\
\hline $115.98(9)$ & $\mathrm{O} 1-\mathrm{C} 25-\mathrm{C} 16$ & $104.57(19)$ \\
\hline $102.41(11)$ & $\mathrm{O} 1-\mathrm{C} 25-\mathrm{H} 25$ & 109.7 \\
\hline $103.76(11)$ & $\mathrm{O} 1-\mathrm{C} 25-\mathrm{C} 28$ & $109.2(2)$ \\
\hline $113.02(9)$ & $\mathrm{C} 16-\mathrm{C} 25-\mathrm{H} 25$ & 109.7 \\
\hline $116.3(2)$ & $\mathrm{C} 16-\mathrm{C} 25-\mathrm{C} 28$ & $113.8(2)$ \\
\hline $130.16(13)$ & $\mathrm{C} 28-\mathrm{C} 25-\mathrm{H} 25$ & 109.7 \\
\hline $69.34(13)$ & $\mathrm{O} 1-\mathrm{C} 26-\mathrm{C} 27$ & $111.4(2)$ \\
\hline $128.1(2)$ & $\mathrm{O} 2-\mathrm{C} 26-\mathrm{O} 1$ & $123.9(3)$ \\
\hline $70.20(14)$ & $\mathrm{O} 2-\mathrm{C} 26-\mathrm{C} 27$ & $124.6(3)$ \\
\hline $124.18(19)$ & $\mathrm{C} 26-\mathrm{C} 27-\mathrm{H} 27 \mathrm{~A}$ & 109.5 \\
\hline
\end{tabular}




\begin{tabular}{|c|c|c|c|}
\hline $\mathrm{C} 5-\mathrm{C} 1-\mathrm{C} 2$ & $107.5(2)$ & $\mathrm{C} 26-\mathrm{C} 27-\mathrm{H} 27 \mathrm{~B}$ & 109.5 \\
\hline $\mathrm{C} 1-\mathrm{C} 2-\mathrm{Fe} 1$ & $68.93(14)$ & $\mathrm{C} 26-\mathrm{C} 27-\mathrm{H} 27 \mathrm{C}$ & 109.5 \\
\hline $\mathrm{C} 1-\mathrm{C} 2-\mathrm{C} 11$ & $126.4(2)$ & $\mathrm{H} 27 \mathrm{~A}-\mathrm{C} 27-\mathrm{H} 27 \mathrm{~B}$ & 109.5 \\
\hline $\mathrm{C} 3-\mathrm{C} 2-\mathrm{Fe} 1$ & $69.71(15)$ & $\mathrm{H} 27 \mathrm{~A}-\mathrm{C} 27-\mathrm{H} 27 \mathrm{C}$ & 109.5 \\
\hline $\mathrm{C} 3-\mathrm{C} 2-\mathrm{C} 1$ & $107.3(2)$ & $\mathrm{H} 27 \mathrm{~B}-\mathrm{C} 27-\mathrm{H} 27 \mathrm{C}$ & 109.5 \\
\hline $\mathrm{C} 3-\mathrm{C} 2-\mathrm{C} 11$ & $126.2(2)$ & $\mathrm{C} 25-\mathrm{C} 28-\mathrm{H} 28 \mathrm{~A}$ & 109.5 \\
\hline $\mathrm{C} 11-\mathrm{C} 2-\mathrm{Fe} 1$ & $123.68(18)$ & $\mathrm{C} 25-\mathrm{C} 28-\mathrm{H} 28 \mathrm{~B}$ & 109.5 \\
\hline $\mathrm{Fe} 1-\mathrm{C} 3-\mathrm{H} 3$ & 126.2 & $\mathrm{C} 25-\mathrm{C} 28-\mathrm{H} 28 \mathrm{C}$ & 109.5 \\
\hline $\mathrm{C} 2-\mathrm{C} 3-\mathrm{Fe} 1$ & $69.26(14)$ & $\mathrm{H} 28 \mathrm{~A}-\mathrm{C} 28-\mathrm{H} 28 \mathrm{~B}$ & 109.5 \\
\hline $\mathrm{C} 2-\mathrm{C} 3-\mathrm{H} 3$ & 125.7 & $\mathrm{H} 28 \mathrm{~A}-\mathrm{C} 28-\mathrm{H} 28 \mathrm{C}$ & 109.5 \\
\hline $\mathrm{C} 4-\mathrm{C} 3-\mathrm{Fe} 1$ & $70.42(15)$ & $\mathrm{H} 28 \mathrm{~B}-\mathrm{C} 28-\mathrm{H} 28 \mathrm{C}$ & 109.5 \\
\hline $\mathrm{C} 4-\mathrm{C} 3-\mathrm{C} 2$ & $108.7(2)$ & $\mathrm{C} 35-\mathrm{C} 34-\mathrm{P} 1$ & $121.37(19)$ \\
\hline $\mathrm{C} 4-\mathrm{C} 3-\mathrm{H} 3$ & 125.7 & $\mathrm{C} 35-\mathrm{C} 34-\mathrm{C} 39$ & $119.6(2)$ \\
\hline $\mathrm{Fe} 1-\mathrm{C} 4-\mathrm{H} 4$ & 127.2 & $\mathrm{C} 39-\mathrm{C} 34-\mathrm{P} 1$ & $118.9(2)$ \\
\hline $\mathrm{C} 3-\mathrm{C} 4-\mathrm{Fe} 1$ & $69.10(16)$ & $\mathrm{C} 34-\mathrm{C} 35-\mathrm{H} 35$ & 119.8 \\
\hline $\mathrm{C} 3-\mathrm{C} 4-\mathrm{H} 4$ & 125.7 & $\mathrm{C} 34-\mathrm{C} 35-\mathrm{C} 36$ & $120.3(2)$ \\
\hline $\mathrm{C} 3-\mathrm{C} 4-\mathrm{C} 5$ & $108.6(3)$ & $\mathrm{C} 36-\mathrm{C} 35-\mathrm{H} 35$ & 119.8 \\
\hline $\mathrm{C} 5-\mathrm{C} 4-\mathrm{Fe} 1$ & $69.56(15)$ & $\mathrm{C} 35-\mathrm{C} 36-\mathrm{H} 36$ & 120.2 \\
\hline $\mathrm{C} 5-\mathrm{C} 4-\mathrm{H} 4$ & 125.7 & $\mathrm{C} 37-\mathrm{C} 36-\mathrm{C} 35$ & $119.5(3)$ \\
\hline $\mathrm{Fe} 1-\mathrm{C} 5-\mathrm{H} 5$ & 127.1 & $\mathrm{C} 37-\mathrm{C} 36-\mathrm{H} 36$ & 120.2 \\
\hline $\mathrm{C} 1-\mathrm{C} 5-\mathrm{Fe} 1$ & $68.47(14)$ & $\mathrm{C} 36-\mathrm{C} 37-\mathrm{H} 37$ & 119.8 \\
\hline $\mathrm{C} 1-\mathrm{C} 5-\mathrm{H} 5$ & 126.0 & $\mathrm{C} 38-\mathrm{C} 37-\mathrm{C} 36$ & $120.4(3)$ \\
\hline $\mathrm{C} 4-\mathrm{C} 5-\mathrm{Fe} 1$ & $69.99(15)$ & $\mathrm{C} 38-\mathrm{C} 37-\mathrm{H} 37$ & 119.8 \\
\hline $\mathrm{C} 4-\mathrm{C} 5-\mathrm{C} 1$ & $107.9(2)$ & $\mathrm{C} 37-\mathrm{C} 38-\mathrm{H} 38$ & 119.8 \\
\hline $\mathrm{C} 4-\mathrm{C} 5-\mathrm{H} 5$ & 126.0 & $\mathrm{C} 37-\mathrm{C} 38-\mathrm{C} 39$ & $120.3(3)$ \\
\hline $\mathrm{Fe} 1-\mathrm{C} 6-\mathrm{H} 6$ & 126.5 & $\mathrm{C} 39-\mathrm{C} 38-\mathrm{H} 38$ & 119.8 \\
\hline $\mathrm{C} 7-\mathrm{C} 6-\mathrm{Fe} 1$ & $69.49(16)$ & C $34-\mathrm{C} 39-\mathrm{H} 39$ & 120.1 \\
\hline $\mathrm{C} 7-\mathrm{C} 6-\mathrm{H} 6$ & 126.1 & $\mathrm{C} 38-\mathrm{C} 39-\mathrm{C} 34$ & $119.8(3)$ \\
\hline $\mathrm{C} 7-\mathrm{C} 6-\mathrm{C} 10$ & $107.9(3)$ & $\mathrm{C} 38-\mathrm{C} 39-\mathrm{H} 39$ & 120.1 \\
\hline $\mathrm{Fe} 1-\mathrm{C} 1-\mathrm{C} 2-\mathrm{C} 3$ & $59.38(17)$ & $\mathrm{C} 5-\mathrm{C} 1-\mathrm{C} 2-\mathrm{C} 11$ & $-177.1(2)$ \\
\hline $\mathrm{Fe} 1-\mathrm{C} 1-\mathrm{C} 2-\mathrm{C} 11$ & $-117.0(3)$ & $\mathrm{C} 6-\mathrm{C} 7-\mathrm{C} 8-\mathrm{Fe} 1$ & $59.95(19)$ \\
\hline $\mathrm{Fe} 1-\mathrm{C} 1-\mathrm{C} 5-\mathrm{C} 4$ & $-59.07(18)$ & $\mathrm{C} 6-\mathrm{C} 7-\mathrm{C} 8-\mathrm{C} 9$ & $0.5(3)$ \\
\hline $\mathrm{Fe} 1-\mathrm{C} 2-\mathrm{C} 3-\mathrm{C} 4$ & $59.58(18)$ & $\mathrm{C} 7-\mathrm{C} 6-\mathrm{C} 10-\mathrm{Fe} 1$ & $-59.12(18)$ \\
\hline $\mathrm{Fe} 1-\mathrm{C} 2-\mathrm{C} 11-\mathrm{C} 12$ & $110.9(3)$ & $\mathrm{C} 7-\mathrm{C} 6-\mathrm{C} 10-\mathrm{C} 9$ & $-0.3(3)$ \\
\hline $\mathrm{Fe} 1-\mathrm{C} 3-\mathrm{C} 4-\mathrm{C} 5$ & $58.48(18)$ & $\mathrm{C} 7-\mathrm{C} 8-\mathrm{C} 9-\mathrm{Fe} 1$ & $59.88(18)$ \\
\hline $\mathrm{Fe} 1-\mathrm{C} 4-\mathrm{C} 5-\mathrm{C} 1$ & $58.13(17)$ & $\mathrm{C} 7-\mathrm{C} 8-\mathrm{C} 9-\mathrm{C} 10$ & $-0.6(3)$ \\
\hline $\mathrm{Fe} 1-\mathrm{C} 6-\mathrm{C} 7-\mathrm{C} 8$ & $-59.30(19)$ & $\mathrm{C} 8-\mathrm{C} 9-\mathrm{C} 10-\mathrm{Fe} 1$ & $60.26(19)$ \\
\hline $\mathrm{Fe} 1-\mathrm{C} 6-\mathrm{C} 10-\mathrm{C} 9$ & $58.86(19)$ & $\mathrm{C} 8-\mathrm{C} 9-\mathrm{C} 10-\mathrm{C} 6$ & $0.6(3)$ \\
\hline $\mathrm{Fe} 1-\mathrm{C} 7-\mathrm{C} 8-\mathrm{C} 9$ & $-59.48(19)$ & $\mathrm{C} 10-\mathrm{C} 6-\mathrm{C} 7-\mathrm{Fe} 1$ & $59.17(19)$ \\
\hline $\mathrm{Fe} 1-\mathrm{C} 8-\mathrm{C} 9-\mathrm{C} 10$ & $-60.52(19)$ & $\mathrm{C} 10-\mathrm{C} 6-\mathrm{C} 7-\mathrm{C} 8$ & $-0.1(3)$ \\
\hline $\mathrm{Fe} 1-\mathrm{C} 9-\mathrm{C} 10-\mathrm{C} 6$ & $-59.70(19)$ & $\mathrm{C} 11-\mathrm{C} 2-\mathrm{C} 3-\mathrm{Fe} 1$ & $117.5(3)$ \\
\hline $\mathrm{Fe} 2-\mathrm{C} 15-\mathrm{C} 16-\mathrm{C} 17$ & $60.23(16)$ & $\mathrm{C} 11-\mathrm{C} 2-\mathrm{C} 3-\mathrm{C} 4$ & $177.1(2)$ \\
\hline $\mathrm{Fe} 2-\mathrm{C} 15-\mathrm{C} 16-\mathrm{C} 25$ & $-121.5(2)$ & $\mathrm{C} 15-\mathrm{P} 1-\mathrm{C} 1-\mathrm{Fe} 1$ & $177.61(17)$ \\
\hline $\mathrm{Fe} 2-\mathrm{C} 15-\mathrm{C} 19-\mathrm{C} 18$ & $-59.48(19)$ & $\mathrm{C} 15-\mathrm{P} 1-\mathrm{C} 1-\mathrm{C} 2$ & $83.3(2)$ \\
\hline $\mathrm{Fe} 2-\mathrm{C} 16-\mathrm{C} 17-\mathrm{C} 18$ & $58.72(19)$ & $\mathrm{C} 15-\mathrm{P} 1-\mathrm{C} 1-\mathrm{C} 5$ & $-90.3(2)$ \\
\hline $\mathrm{Fe} 2-\mathrm{C} 16-\mathrm{C} 25-\mathrm{O} 1$ & $-172.23(16)$ & $\mathrm{C} 15-\mathrm{P} 1-\mathrm{C} 34-\mathrm{C} 35$ & $24.6(2)$ \\
\hline $\mathrm{Fe} 2-\mathrm{C} 16-\mathrm{C} 25-\mathrm{C} 28$ & $68.7(3)$ & $\mathrm{C} 15-\mathrm{P} 1-\mathrm{C} 34-\mathrm{C} 39$ & $-158.1(2)$ \\
\hline
\end{tabular}




\begin{tabular}{|c|c|}
\hline $\mathrm{Fe} 2-\mathrm{C} 17-\mathrm{C} 18-\mathrm{C} 19$ & $58.81(19)$ \\
\hline $\mathrm{Fe} 2-\mathrm{C} 18-\mathrm{C} 19-\mathrm{C} 15$ & $58.97(18)$ \\
\hline $\mathrm{Fe} 2-\mathrm{C} 20-\mathrm{C} 21-\mathrm{C} 22$ & $-59.15(19)$ \\
\hline $\mathrm{Fe} 2-\mathrm{C} 20-\mathrm{C} 24-\mathrm{C} 23$ & $59.1(2)$ \\
\hline $\mathrm{Fe} 2-\mathrm{C} 21-\mathrm{C} 22-\mathrm{C} 23$ & $-59.8(2)$ \\
\hline $\mathrm{Fe} 2-\mathrm{C} 22-\mathrm{C} 23-\mathrm{C} 24$ & $-59.5(2)$ \\
\hline $\mathrm{Fe} 2-\mathrm{C} 23-\mathrm{C} 24-\mathrm{C} 20$ & $-59.3(2)$ \\
\hline $\mathrm{S} 1-\mathrm{P} 1-\mathrm{C} 1-\mathrm{Fe} 1$ & $49.8(2)$ \\
\hline $\mathrm{S} 1-\mathrm{P} 1-\mathrm{C} 1-\mathrm{C} 2$ & $-44.5(2)$ \\
\hline $\mathrm{S} 1-\mathrm{P} 1-\mathrm{C} 1-\mathrm{C} 5$ & $141.96(19)$ \\
\hline $\mathrm{S} 1-\mathrm{P} 1-\mathrm{C} 15-\mathrm{Fe} 2$ & $42.7(2)$ \\
\hline $\mathrm{S} 1-\mathrm{P} 1-\mathrm{C} 15-\mathrm{C} 16$ & $-53.7(2)$ \\
\hline $\mathrm{S} 1-\mathrm{P} 1-\mathrm{C} 15-\mathrm{C} 19$ & $135.49(19)$ \\
\hline $\mathrm{S} 1-\mathrm{P} 1-\mathrm{C} 34-\mathrm{C} 35$ & $151.04(18)$ \\
\hline $\mathrm{S} 1-\mathrm{P} 1-\mathrm{C} 34-\mathrm{C} 39$ & $-31.7(2)$ \\
\hline $\mathrm{P} 1-\mathrm{C} 1-\mathrm{C} 2-\mathrm{Fe} 1$ & $125.5(2)$ \\
\hline $\mathrm{P} 1-\mathrm{C} 1-\mathrm{C} 2-\mathrm{C} 3$ & $-175.15(18$ \\
\hline $\mathrm{P} 1-\mathrm{C} 1-\mathrm{C} 2-\mathrm{C} 11$ & $8.5(4)$ \\
\hline $\mathrm{P} 1-\mathrm{C} 1-\mathrm{C} 5-\mathrm{Fe} 1$ & $-125.74(19$ \\
\hline $\mathrm{P} 1-\mathrm{C} 1-\mathrm{C} 5-\mathrm{C} 4$ & $175.19(18)$ \\
\hline $\mathrm{P} 1-\mathrm{C} 15-\mathrm{C} 16-\mathrm{Fe} 2$ & $128.8(2)$ \\
\hline $\mathrm{P} 1-\mathrm{C} 15-\mathrm{C} 16-\mathrm{C} 17$ & $-171.0(2)$ \\
\hline $\mathrm{P} 1-\mathrm{C} 15-\mathrm{C} 16-\mathrm{C} 25$ & $7.3(4)$ \\
\hline $\mathrm{P} 1-\mathrm{C} 15-\mathrm{C} 19-\mathrm{Fe} 2$ & $-128.71(19$ \\
\hline $\mathrm{P} 1-\mathrm{C} 15-\mathrm{C} 19-\mathrm{C} 18$ & $171.81(19)$ \\
\hline $\mathrm{P} 1-\mathrm{C} 34-\mathrm{C} 35-\mathrm{C} 36$ & $178.1(2)$ \\
\hline $\mathrm{P} 1-\mathrm{C} 34-\mathrm{C} 39-\mathrm{C} 38$ & $-176.8(2)$ \\
\hline $\mathrm{C} 1-\mathrm{P} 1-\mathrm{C} 15-\mathrm{Fe} 2$ & $-85.5(2)$ \\
\hline $\mathrm{C} 1-\mathrm{P} 1-\mathrm{C} 15-\mathrm{C} 16$ & $178.1(2)$ \\
\hline $\mathrm{C} 1-\mathrm{P} 1-\mathrm{C} 15-\mathrm{C} 19$ & $7.2(2)$ \\
\hline $\mathrm{C} 1-\mathrm{P} 1-\mathrm{C} 34-\mathrm{C} 35$ & $-82.0(2)$ \\
\hline $\mathrm{C} 1-\mathrm{P} 1-\mathrm{C} 34-\mathrm{C} 39$ & $95.3(2)$ \\
\hline $\mathrm{C} 1-\mathrm{C} 2-\mathrm{C} 3-\mathrm{Fe} 1$ & $-58.89(16)$ \\
\hline $\mathrm{C} 1-\mathrm{C} 2-\mathrm{C} 3-\mathrm{C} 4$ & $0.7(3)$ \\
\hline $\mathrm{C} 1-\mathrm{C} 2-\mathrm{C} 11-\mathrm{C} 12$ & $-161.7(3)$ \\
\hline $\mathrm{C} 2-\mathrm{C} 1-\mathrm{C} 5-\mathrm{Fe} 1$ & $59.56(16)$ \\
\hline $\mathrm{C} 2-\mathrm{C} 1-\mathrm{C} 5-\mathrm{C} 4$ & $0.5(3)$ \\
\hline $\mathrm{C} 2-\mathrm{C} 3-\mathrm{C} 4-\mathrm{Fe} 1$ & $-58.87(18)$ \\
\hline $\mathrm{C} 2-\mathrm{C} 3-\mathrm{C} 4-\mathrm{C} 5$ & $-0.4(3)$ \\
\hline $\mathrm{C} 3-\mathrm{C} 2-\mathrm{C} 11-\mathrm{C} 12$ & $22.6(4)$ \\
\hline $\mathrm{C} 3-\mathrm{C} 4-\mathrm{C} 5-\mathrm{Fe} 1$ & $-58.20(18)$ \\
\hline $\mathrm{C} 3-\mathrm{C} 4-\mathrm{C} 5-\mathrm{C} 1$ & $-0.1(3)$ \\
\hline $\mathrm{C} 5-\mathrm{C} 1-\mathrm{C} 2-\mathrm{Fe} 1$ & $-60.11(16)$ \\
\hline $\mathrm{C} 5-\mathrm{C} 1-\mathrm{C} 2-\mathrm{C} 3$ & $-0.7(3)$ \\
\hline
\end{tabular}

$\begin{array}{ll}\mathrm{C} 15-\mathrm{C} 16-\mathrm{C} 17-\mathrm{Fe} 2 & -59.79(16) \\ \mathrm{C} 15-\mathrm{C} 16-\mathrm{C} 17-\mathrm{C} 18 & -1.1(3) \\ \mathrm{C} 15-\mathrm{C} 16-\mathrm{C} 25-\mathrm{O} 1 & -81.0(3) \\ \mathrm{C} 15-\mathrm{C} 16-\mathrm{C} 25-\mathrm{C} 28 & 160.0(2) \\ \mathrm{C} 16-\mathrm{C} 15-\mathrm{C} 19-\mathrm{F} 22 & 58.83(16) \\ \mathrm{C} 16-\mathrm{C} 15-\mathrm{C} 19-\mathrm{C} 18 & -0.6(3) \\ \mathrm{C} 16-\mathrm{C} 17-\mathrm{C} 18-\mathrm{F} 2 & -58.13(18) \\ \mathrm{C} 16-\mathrm{C} 17-\mathrm{C} 18-\mathrm{C} 19 & 0.7(3) \\ \mathrm{C} 17-\mathrm{C} 16-\mathrm{C} 25-\mathrm{O} 1 & 97.0(3) \\ \mathrm{C} 17-\mathrm{C} 16-\mathrm{C} 25-\mathrm{C} 28 & -22.0(3) \\ \mathrm{C} 17-\mathrm{C} 18-\mathrm{C} 19-\mathrm{F} 2 & -58.99(19) \\ \mathrm{C} 17-\mathrm{C} 18-\mathrm{C} 19-\mathrm{C} 15 & 0.0(3) \\ \mathrm{C} 19-\mathrm{C} 15-\mathrm{C} 16-\mathrm{F} 22 & -59.18(17) \\ \mathrm{C} 19-\mathrm{C} 15-\mathrm{C} 16-\mathrm{C} 17 & 1.0(3) \\ \mathrm{C} 19-\mathrm{C} 15-\mathrm{C} 16-\mathrm{C} 25 & 179.3(2) \\ \mathrm{C} 20-\mathrm{C} 21-\mathrm{C} 22-\mathrm{Fe} 2 & 59.62(19) \\ \mathrm{C} 20-\mathrm{C} 21-\mathrm{C} 22-\mathrm{C} 23 & -0.1(3) \\ \mathrm{C} 21-\mathrm{C} 20-\mathrm{C} 24-\mathrm{Fe} 2 & -59.1(2) \\ \mathrm{C} 21-\mathrm{C} 20-\mathrm{C} 24-\mathrm{C} 23 & 0.0(3) \\ \mathrm{C} 21-\mathrm{C} 22-\mathrm{C} 23-\mathrm{F} 2 & 59.68(19) \\ \mathrm{C} 21-\mathrm{C} 22-\mathrm{C} 23-\mathrm{C} 24 & 0.1(3) \\ \mathrm{C} 22-\mathrm{C} 23-\mathrm{C} 24-\mathrm{Fe} 2 & 59.2(2) \\ \mathrm{C} 22-\mathrm{C} 23-\mathrm{C} 24-\mathrm{C} 20 & -0.1(4) \\ \mathrm{C} 24-\mathrm{C} 20-\mathrm{C} 21-\mathrm{Fe} 2 & 59.2(2) \\ \mathrm{C} 24-\mathrm{C} 20-\mathrm{C} 21-\mathrm{C} 22 & 0.1(3) \\ \mathrm{C} 25-\mathrm{O} 1-\mathrm{C} 26-\mathrm{O} 2 & 1.8(3) \\ \mathrm{C} 25-\mathrm{O} 1-\mathrm{C} 26-\mathrm{C} 27 & -178.7(2) \\ \mathrm{C} 25-\mathrm{C} 16-\mathrm{C} 17-\mathrm{Fe} 2 & 121.9(2) \\ \mathrm{C} 25-\mathrm{C} 16-\mathrm{C} 17-\mathrm{C} 18 & -179.4(2) \\ \mathrm{C} 26-\mathrm{O} 1-\mathrm{C} 25-\mathrm{C} 16 & 157.5(2) \\ \mathrm{C} 26-\mathrm{O} 1-\mathrm{C} 25-\mathrm{C} 28 & -80.4(3) \\ \mathrm{C} 34-\mathrm{P} 1-\mathrm{C} 1-\mathrm{Fe} 1 & -74.80(19) \\ \mathrm{C} 34-\mathrm{P} 1-\mathrm{C} 1-\mathrm{C} 2 & -169.1(2) \\ \mathrm{C} 34-\mathrm{P} 1-\mathrm{C} 1-\mathrm{C} 5 & 17.3(2) \\ \mathrm{C} 34-\mathrm{P} 1-\mathrm{C} 15-\mathrm{Fe} 2 & 167.25(17) \\ \mathrm{C} 34-\mathrm{P} 1-\mathrm{C} 15-\mathrm{C} 16 & 70.9(2) \\ \mathrm{C} 34-\mathrm{P} 1-\mathrm{C} 15-\mathrm{C} 19 & -100.0(2) \\ \mathrm{C} 34-\mathrm{C} 35-\mathrm{C} 36-\mathrm{C} 37 & -1.5(4) \\ \mathrm{C} 35-\mathrm{C} 34-\mathrm{C} 39-\mathrm{C} 38 & 0.5(4) \\ \mathrm{C} 35-\mathrm{C} 36-\mathrm{C} 37-\mathrm{C} 38 & 0.8(4) \\ \mathrm{C} 36-\mathrm{C} 37-\mathrm{C} 38-\mathrm{C} 39 & 0.6(4) \\ \mathrm{C} 37-\mathrm{C} 38-\mathrm{C} 39-\mathrm{C} 34 & -1.3(4) \\ \mathrm{C} 39-\mathrm{C} 34-\mathrm{C} 35-\mathrm{C} 36 & 0.9(4) \\ & \end{array}$

$-59.79(16)$

$-81.0(3)$

$160.0(2)$

$58.83(16)$

$-0.6(3)$

$-58.13(18)$

0.7 (3)

$97.0(3)$

$-22.0(3)$

$0.0(3)$

$-59.18(17)$

$1.0(3)$

$179.3(2)$

$59.62(19)$

$-0.1(3)$

$-59.1(2)$

$0.0(3)$

$59.68(19)$

0.1 (3)

$59.2(2)$

$-0.1(4)$

$0.2(2)$

$-178.7(2)$

$21.9(2)$

$-179.4(2)$

$157.5(2)$

$-80.4(3)$

$-74.80(19)$

$-169.1(2)$

$17.3(2)$

167.25 (17)

$70.9(2)$

$-100.0(2)$

$0.5(4)$

0.8 (4)

$-1.3(4)$

0.9 (4) 
[(2S $)$-2-Ethenylferrocen-1-yl] $\left\{\left(2 S_{\mathrm{p}}\right)-2-[(1 R)-1\right.$-hydroxyethyl]ferrocen-1-yl $\}$ phenyl-(S)-phosphane sulfide (8b)

Crystal data

$\left[\mathrm{Fe}_{2}\left(\mathrm{C}_{5} \mathrm{H}_{5}\right)_{2}\left(\mathrm{C}_{20} \mathrm{H}_{19} \mathrm{OPS}\right)\right]$

$M_{r}=580.26$

Orthorhombic, $P 2{ }_{1} 2_{1} 2_{1}$

$a=7.5285(3) \AA$

$b=17.6463(7) \AA$

$c=39.3333(15) \AA$

$V=5225.4(4) \AA^{3}$

$Z=8$

$F(000)=2400$

\section{Data collection}

Bruker X8 APEXII

diffractometer

Radiation source: sealed xray tube, Incoatec IuS $\varphi$ and $\omega$ scans

Absorption correction: multi-scan

(SADABS; Bruker, 2008)

$T_{\min }=0.650, T_{\max }=0.746$

38763 measured reflections

\section{Refinement}

Refinement on $F^{2}$

Least-squares matrix: full

$R\left[F^{2}>2 \sigma\left(F^{2}\right)\right]=0.056$

$w R\left(F^{2}\right)=0.137$

$S=1.04$

14944 reflections

635 parameters

1 restraint

Primary atom site location: structure-invariant direct methods
$D_{\mathrm{x}}=1.475 \mathrm{Mg} \mathrm{m}^{-3}$

Mo $K \alpha$ radiation, $\lambda=0.71073 \AA$

Cell parameters from 9990 reflections

$\theta=2.4-29.3^{\circ}$

$\mu=1.27 \mathrm{~mm}^{-1}$

$T=130 \mathrm{~K}$

Block, clear orange

$0.25 \times 0.2 \times 0.17 \mathrm{~mm}$

14944 independent reflections

11780 reflections with $I>2 \sigma(I)$

$R_{\text {int }}=0.045$

$\theta_{\max }=30.0^{\circ}, \theta_{\min }=1.0^{\circ}$

$h=-9 \rightarrow 10$

$k=-24 \rightarrow 24$

$l=-49 \rightarrow 55$

Hydrogen site location: inferred from neighbouring sites

$\mathrm{H}$-atom parameters constrained

$w=1 /\left[\sigma^{2}\left(F_{\mathrm{o}}^{2}\right)+(0.0521 P)^{2}+6.896 P\right]$

where $P=\left(F_{\mathrm{o}}^{2}+2 F_{\mathrm{c}}{ }^{2}\right) / 3$

$(\Delta / \sigma)_{\max }=0.001$

$\Delta \rho_{\max }=2.08 \mathrm{e} \AA^{-3}$

$\Delta \rho_{\min }=-1.80$ e $\AA^{-3}$

Absolute structure: Flack $x$ determined using 4140 quotients $[(\mathrm{I}+)-(\mathrm{I}-)] /[(\mathrm{I}+)+(\mathrm{I}-)]$ (Parsons et al., 2013)

Absolute structure parameter: -0.010 (7)

\section{Special details}

Geometry. All esds (except the esd in the dihedral angle between two 1.s. planes) are estimated using the full covariance matrix. The cell esds are taken into account individually in the estimation of esds in distances, angles and torsion angles; correlations between esds in cell parameters are only used when they are defined by crystal symmetry. An approximate (isotropic) treatment of cell esds is used for estimating esds involving l.s. planes.

Refinement._olex2_refinement_description 1. Fixed Uiso At 1.2 times of: All $\mathrm{C}(\mathrm{H})$ groups, $\mathrm{All} \mathrm{C}(\mathrm{H}, \mathrm{H})$ groups At 1.5 times of: $\mathrm{All} \mathrm{C}(\mathrm{H}, \mathrm{H}, \mathrm{H})$ groups, $\mathrm{All} \mathrm{O}(\mathrm{H})$ groups 2. Restrained distances H1B-C34B 2.5 with sigma of 0.05 3.a Ternary $\mathrm{CH}$ refined with riding coordinates: C25A(H25A), C25B(H25B) 3.b Aromatic/amide $\mathrm{H}$ refined with riding coordinates: C3A(H3A), C4A(H4A), C5A(H5A), C6A(H6A), C7A(H7A), C8A(H8A), C9A(H9A), C10A(H10A), C11A(H11A), C17A(H17A), C18A(H18A), C19A(H19A), C20A(H20A), C21A(H21A), C22A(H22A), C23A(H23A), C24A(H24A), C35A(H35A), C36A(H36A), C37A(H37A), C38A(H38A), C39A(H39A), C3B(H3B), C4B(H4B), C5B(H5B), C6B(H6B), C7B(H7B), C8B(H8B), C9B(H9B), C10B(H10B), C11B(H11B), C17B(H17B), C18B(H18B), C19B(H19B), C20B(H20B), C21B(H21B), C22B(H22B), C23B(H23B), C24B(H24B), C35B(H35B), C36B(H36B), C37B(H37B), C38B(H38B), C39B(H39B) 3.c $\mathrm{X}=\mathrm{CH} 2$ refined with riding coordinates: $\mathrm{C} 12 \mathrm{~A}(\mathrm{H} 12 \mathrm{~A}, \mathrm{H} 12 \mathrm{~B}), \mathrm{C} 12 \mathrm{~B}(\mathrm{H} 12 \mathrm{C}, \mathrm{H} 12 \mathrm{D})$ 3.d Idealised Me refined as rotating group: $\mathrm{C} 26 \mathrm{~A}(\mathrm{H} 26 \mathrm{~A}, \mathrm{H} 26 \mathrm{~B}, \mathrm{H} 26 \mathrm{C}), \mathrm{C} 26 \mathrm{~B}(\mathrm{H} 26 \mathrm{D}, \mathrm{H} 26 \mathrm{E}, \mathrm{H} 26 \mathrm{~F})$ 3.e Idealised tetrahedral $\mathrm{OH}$ refined as rotating group: $\mathrm{O} 1 \mathrm{~A}(\mathrm{H} 1 \mathrm{~A}), \mathrm{O} 1 \mathrm{~B}(\mathrm{H} 1 \mathrm{~B})$ 
Fractional atomic coordinates and isotropic or equivalent isotropic displacement parameters $\left(\AA^{2}\right)$

\begin{tabular}{|c|c|c|c|c|}
\hline & $x$ & $y$ & $z$ & $U_{\text {iso }} * / U_{\text {eq }}$ \\
\hline FelA & $0.72689(11)$ & $0.58337(4)$ & $0.56427(2)$ & $0.02723(17)$ \\
\hline $\mathrm{Fe} 2 \mathrm{~A}$ & $0.90817(12)$ & $0.50676(5)$ & $0.41673(2)$ & $0.03364(19)$ \\
\hline S1A & $0.4437(2)$ & $0.55690(8)$ & $0.47076(4)$ & $0.0329(3)$ \\
\hline P1A & $0.63867(18)$ & $0.49477(7)$ & $0.48811(3)$ & $0.0215(2)$ \\
\hline O1A & $0.4921(7)$ & $0.3319(3)$ & $0.42896(12)$ & $0.0496(12)$ \\
\hline H1A & 0.481829 & 0.339883 & 0.449923 & $0.074^{*}$ \\
\hline $\mathrm{C} 1 \mathrm{~A}$ & $0.7859(7)$ & 0.5377 & $0.51848(12)$ & $0.0249(10)$ \\
\hline $\mathrm{C} 2 \mathrm{~A}$ & $0.8462(8)$ & 0.6159 & $0.52000(13)$ & $0.0319(12)$ \\
\hline $\mathrm{C} 3 \mathrm{~A}$ & $0.9682(9)$ & $0.6202(4)$ & $0.54785(15)$ & 0.0399 (14) \\
\hline $\mathrm{H} 3 \mathrm{~A}$ & 1.027855 & 0.664803 & 0.555144 & $0.048 *$ \\
\hline $\mathrm{C} 4 \mathrm{~A}$ & $0.9862(8)$ & $0.5476(4)$ & $0.56284(15)$ & 0.0368 (13) \\
\hline $\mathrm{H} 4 \mathrm{~A}$ & 1.060591 & 0.535142 & 0.581538 & $0.044 *$ \\
\hline C5A & $0.8742(7)$ & $0.4968(3)$ & $0.54515(13)$ & $0.0294(11)$ \\
\hline H5A & 0.859724 & 0.444446 & 0.550079 & $0.035^{*}$ \\
\hline C6A & $0.4564(8)$ & 0.5854 & $0.56956(14)$ & $0.0354(12)$ \\
\hline H6A & 0.371524 & 0.568517 & 0.553370 & $0.042 *$ \\
\hline C7A & $0.5267(9)$ & $0.6602(3)$ & $0.57211(15)$ & $0.0388(14)$ \\
\hline H7A & 0.497184 & 0.701982 & 0.557971 & $0.047^{*}$ \\
\hline C8A & $0.6488(9)$ & $0.6610(3)$ & $0.59955(15)$ & $0.0388(14)$ \\
\hline H8A & 0.715712 & 0.703531 & 0.606930 & $0.047^{*}$ \\
\hline C9A & $0.6541(8)$ & $0.5874(4)$ & $0.61417(14)$ & $0.0362(12)$ \\
\hline H9A & 0.724229 & 0.572079 & 0.633029 & $0.043^{*}$ \\
\hline $\mathrm{C} 10 \mathrm{~A}$ & $0.5349(8)$ & 0.5409 & $0.59534(14)$ & 0.0355 \\
\hline $\mathrm{H} 10 \mathrm{~A}$ & 0.512097 & 0.488778 & 0.599418 & $0.043^{*}$ \\
\hline C11A & $0.7839(10)$ & $0.6800(3)$ & $0.49998(16)$ & $0.0434(15)$ \\
\hline H11A & 0.702283 & 0.669007 & 0.482224 & $0.052 *$ \\
\hline C12A & $0.8284(12)$ & $0.7509(4)$ & 0.5039 & $0.063(2)$ \\
\hline $\mathrm{H} 12 \mathrm{~A}$ & 0.909615 & 0.764937 & 0.521286 & $0.076^{*}$ \\
\hline H12B & 0.779709 & 0.788540 & 0.489354 & $0.076^{*}$ \\
\hline C15A & $0.7888(7)$ & 0.4568 & $0.45709(12)$ & $0.0228(9)$ \\
\hline C16A & $0.7459(7)$ & $0.4173(3)$ & $0.42600(12)$ & $0.0267(10)$ \\
\hline C17A & $0.9091(8)$ & $0.3920(3)$ & $0.41194(14)$ & $0.0342(12)$ \\
\hline H17A & 0.920726 & 0.364127 & 0.391387 & $0.041 *$ \\
\hline C18A & $1.0508(8)$ & $0.4141(3)$ & $0.43292(16)$ & $0.0376(13)$ \\
\hline H18A & 1.172981 & 0.403694 & 0.429057 & $0.045^{*}$ \\
\hline C19A & $0.9793(7)$ & $0.4550(3)$ & $0.46105(14)$ & 0.0307 (11) \\
\hline H19A & 1.045205 & 0.477083 & 0.479122 & $0.037 *$ \\
\hline $\mathrm{C} 20 \mathrm{~A}$ & $0.8220(12)$ & $0.6174(4)$ & $0.41152(19)$ & $0.056(2)$ \\
\hline $\mathrm{H} 20 \mathrm{~A}$ & 0.736712 & 0.642900 & 0.425144 & $0.067^{*}$ \\
\hline $\mathrm{C} 21 \mathrm{~A}$ & $0.7885(12)$ & $0.5742(4)$ & $0.38160(17)$ & $0.059(2)$ \\
\hline $\mathrm{H} 21 \mathrm{~A}$ & 0.674693 & 0.565730 & 0.371867 & $0.070^{*}$ \\
\hline $\mathrm{C} 22 \mathrm{~A}$ & $0.9473(14)$ & $0.5464(5)$ & $0.36890(19)$ & $0.068(3)$ \\
\hline $\mathrm{H} 22 \mathrm{~A}$ & 0.961400 & 0.516048 & 0.349142 & $0.082 *$ \\
\hline $\mathrm{C} 23 \mathrm{~A}$ & $1.0842(12)$ & $0.5715(5)$ & $0.3907(2)$ & $0.067(2)$ \\
\hline $\mathrm{H} 23 \mathrm{~A}$ & 1.207123 & 0.560776 & 0.387988 & $0.080 *$ \\
\hline
\end{tabular}




\begin{tabular}{|c|c|c|c|c|}
\hline $\mathrm{C} 24 \mathrm{~A}$ & $1.0091(13)$ & 0.6148 (4) & $0.4169(2)$ & $0.064(2)$ \\
\hline $\mathrm{H} 24 \mathrm{~A}$ & 1.071804 & 0.638125 & 0.435086 & $0.077^{*}$ \\
\hline $\mathrm{C} 25 \mathrm{~A}$ & $0.5625(9)$ & $0.3995(3)$ & $0.41240(15)$ & 0.0395 (14) \\
\hline $\mathrm{H} 25 \mathrm{~A}$ & 0.482977 & 0.442919 & 0.418328 & $0.047 *$ \\
\hline $\mathrm{C} 26 \mathrm{~A}$ & $0.5476(13)$ & $0.3864(5)$ & $0.37579(18)$ & $0.068(2)$ \\
\hline $\mathrm{H} 26 \mathrm{~A}$ & 0.600296 & 0.429109 & 0.363484 & $0.102 *$ \\
\hline H26B & 0.422028 & 0.381616 & 0.369580 & $0.102 *$ \\
\hline $\mathrm{H} 26 \mathrm{C}$ & 0.610430 & 0.339620 & 0.369791 & $0.102^{*}$ \\
\hline $\mathrm{C} 34 \mathrm{~A}$ & $0.5525(8)$ & $0.4108(3)$ & $0.50942(12)$ & $0.0263(10)$ \\
\hline C35A & $0.6566(9)$ & $0.3457(3)$ & $0.51330(14)$ & $0.0356(13)$ \\
\hline $\mathrm{H} 35 \mathrm{~A}$ & 0.774718 & 0.344963 & 0.504835 & $0.043 *$ \\
\hline C36A & $0.5878(11)$ & $0.2816(3)$ & $0.52957(15)$ & $0.0460(17)$ \\
\hline H36A & 0.659011 & 0.237537 & 0.532309 & $0.055^{*}$ \\
\hline C37A & 0.4154 (11) & $0.2828(4)$ & $0.54164(15)$ & 0.0497 (18) \\
\hline H37A & 0.367680 & 0.239471 & 0.552681 & $0.060 *$ \\
\hline C38A & $0.3126(10)$ & 0.3468 (4) & $0.53767(16)$ & $0.0473(17)$ \\
\hline $\mathrm{H} 38 \mathrm{~A}$ & 0.194223 & 0.347044 & 0.546014 & $0.057^{*}$ \\
\hline C39A & $0.3786(8)$ & $0.4105(3)$ & $0.52181(14)$ & $0.0345(12)$ \\
\hline H39A & 0.306022 & 0.454216 & 0.519319 & $0.041 *$ \\
\hline Fe1B & $0.26525(12)$ & $0.28793(6)$ & $0.17616(3)$ & $0.0465(3)$ \\
\hline $\mathrm{Fe} 2 \mathrm{~B}$ & 0.08660 (14) & $0.18732(5)$ & $0.32286(2)$ & $0.0406(2)$ \\
\hline S1B & $0.5324(2)$ & $0.27642(8)$ & $0.27046(4)$ & 0.0373 (3) \\
\hline P1B & 0.36294 (19) & $0.20308(7)$ & $0.25184(4)$ & $0.0276(3)$ \\
\hline O1B & $0.5684(10)$ & $0.0491(7)$ & $0.3061(2)$ & $0.146(5)$ \\
\hline H1B & 0.541816 & 0.050393 & 0.285400 & $0.219^{*}$ \\
\hline C1B & $0.2073(7)$ & $0.2372(3)$ & $0.22072(14)$ & $0.0306(11)$ \\
\hline $\mathrm{C} 2 \mathrm{~B}$ & $0.1185(8)$ & $0.3093(3)$ & $0.21902(16)$ & $0.0376(13)$ \\
\hline $\mathrm{C} 3 \mathrm{~B}$ & $0.0059(8)$ & 0.3084 (4) & 0.18994 (17) & $0.0412(15)$ \\
\hline H3B & -0.068780 & 0.348483 & 0.182527 & $0.049^{*}$ \\
\hline C4B & $0.0253(9)$ & $0.2368(4)$ & 0.17407 (17) & $0.0463(16)$ \\
\hline H4B & -0.034289 & 0.221283 & 0.153948 & $0.056^{*}$ \\
\hline C5B & $0.1462(8)$ & 0.1919 (4) & $0.19258(15)$ & $0.0380(13)$ \\
\hline H5B & 0.180846 & 0.141432 & 0.187474 & $0.046^{*}$ \\
\hline C6B & $0.5317(9)$ & 0.3057 (6) & $0.1728(3)$ & $0.077(3)$ \\
\hline H6B & 0.616455 & 0.294239 & 0.189959 & $0.092 *$ \\
\hline C7B & $0.4419(11)$ & $0.3746(6)$ & $0.1698(2)$ & $0.079(3)$ \\
\hline H7B & 0.453339 & 0.417800 & 0.184019 & $0.095 *$ \\
\hline $\mathrm{C} 8 \mathrm{~B}$ & $0.3284(10)$ & $0.3667(6)$ & $0.1405(2)$ & $0.077(3)$ \\
\hline H8B & 0.250993 & 0.404793 & 0.132038 & $0.092 *$ \\
\hline C9B & $0.3492(12)$ & $0.2946(7)$ & $0.1265(2)$ & $0.076(3)$ \\
\hline H9B & 0.288574 & 0.275542 & 0.107075 & $0.092 *$ \\
\hline $\mathrm{C} 10 \mathrm{~B}$ & $0.4776(12)$ & $0.2543(7)$ & $0.1463(3)$ & $0.083(3)$ \\
\hline H10B & 0.518752 & 0.204057 & 0.142677 & $0.100^{*}$ \\
\hline C11B & $0.1372(10)$ & $0.3735(3)$ & $0.24254(19)$ & $0.0456(16)$ \\
\hline H11B & 0.243928 & 0.378078 & 0.255245 & $0.055^{*}$ \\
\hline $\mathrm{C} 12 \mathrm{~B}$ & $0.0112(13)$ & $0.4253(4)$ & 0.2468 (3) & $0.071(2)$ \\
\hline $\mathrm{H} 12 \mathrm{C}$ & -0.096649 & 0.421812 & 0.234374 & $0.086^{*}$ \\
\hline H12D & 0.028965 & 0.465902 & 0.262315 & $0.086^{*}$ \\
\hline
\end{tabular}




$\begin{array}{lllll}\text { C15B } & 0.2279(8) & 0.1535(3) & 0.28203(14) & 0.0305(11) \\ \text { C16B } & 0.2868(10) & 0.1137(3) & 0.31220(16) & 0.0437(15) \\ \text { C17B } & 0.1361(10) & 0.0741(4) & 0.32479(17) & 0.0471(16) \\ \text { H17B } & 0.135397 & 0.043146 & 0.344550 & 0.056^{*} \\ \text { C18B } & -0.0110(9) & 0.0873(4) & 0.30393(15) & 0.0404(14) \\ \text { H18B } & -0.127006 & 0.067313 & 0.307058 & 0.049^{*} \\ \text { C19B } & 0.0442(8) & 0.1361(3) & 0.27724(14) & 0.0337(12) \\ \text { H19B } & -0.028663 & 0.154085 & 0.259240 & 0.040^{*} \\ \text { C20B } & 0.1274(11) & 0.2999(4) & 0.3314(2) & 0.0533(18) \\ \text { H20B } & 0.207787 & 0.331261 & 0.319193 & 0.064^{*} \\ \text { C21B } & 0.1659(18) & 0.2564(5) & 0.3615(2) & 0.087(4) \\ \text { H21B } & 0.276115 & 0.253832 & 0.373252 & 0.104^{*} \\ \text { C22B } & 0.009(3) & 0.2188(6) & 0.3701(2) & 0.124(7) \\ \text { H22B } & -0.005504 & 0.185628 & 0.388905 & 0.149^{*} \\ \text { C23B } & -0.1198(18) & 0.2371(6) & 0.3472(3) & 0.103(5) \\ \text { H23B } & -0.238357 & 0.218687 & 0.347330 & 0.123^{*} \\ \text { C24B } & -0.0492(12) & 0.2871(5) & 0.3236(2) & 0.062(2) \\ \text { H24B } & -0.112028 & 0.309028 & 0.305134 & 0.074^{*} \\ \text { C25B } & 0.4773(14) & 0.1103(5) & 0.3239(3) & 0.085(3) \\ \text { H25B } & 0.537917 & 0.159761 & 0.319410 & 0.103^{*} \\ \text { C26B } & 0.4888(14) & 0.0901(5) & 0.3619(2) & 0.080(3) \\ \text { H26D } & 0.422548 & 0.127650 & 0.375211 & 0.119^{*} \\ \text { H26E } & 0.613428 & 0.090148 & 0.369085 & 0.119^{*} \\ \text { H26F } & 0.437638 & 0.039739 & 0.365642 & 0.119^{*} \\ \text { C34B } & 0.4751(8) & 0.1251(3) & 0.22994(14) & 0.0297(11) \\ \text { C35B } & 0.3919(9) & 0.0551(3) & 0.22620(16) & 0.0396(13) \\ \text { H35B } & 0.276944 & 0.047290 & 0.235515 & 0.048^{*} \\ \text { C36B } & 0.4760(10) & -0.0031(4) & 0.20895(18) & 0.0487(16) \\ \text { H36B } & 0.420204 & -0.051125 & 0.206828 & 0.058^{*} \\ \text { C37B } & 0.6413(10) & 0.0091(4) & 0.19488(18) & 0.0491(16) \\ \text { H37B } & 0.697762 & -0.030307 & 0.182485 & 0.059^{*} \\ \text { C38B } & 0.7259(10) & 0.0783(4) & 0.19864(17) & 0.0474(15) \\ \text { H38B } & 0.840391 & 0.086177 & 0.189128 & 0.057^{*} \\ \text { C39B } & 0.6420(8) & 0.1360(3) & 0.21639(14) & 0.0344(12) \\ \text { H39B } & 0.699993 & 0.183439 & 0.219237 & 0.041^{*} \\ & & & & \end{array}$

Atomic displacement parameters $\left(\AA^{2}\right)$

\begin{tabular}{lllllll}
\hline & $U^{11}$ & $U^{22}$ & $U^{33}$ & $U^{12}$ & $U^{13}$ & $U^{23}$ \\
\hline Fe1A & $0.0270(4)$ & $0.0313(3)$ & $0.0234(3)$ & $-0.0057(3)$ & $0.0012(3)$ & $-0.0056(3)$ \\
Fe2A & $0.0356(4)$ & $0.0386(4)$ & $0.0267(4)$ & $-0.0072(4)$ & $0.0068(4)$ & $0.0052(3)$ \\
S1A & $0.0298(7)$ & $0.0314(6)$ & $0.0374(7)$ & $0.0090(6)$ & $-0.0027(6)$ & $0.0018(5)$ \\
P1A & $0.0220(6)$ & $0.0218(5)$ & $0.0208(5)$ & $-0.0001(5)$ & $-0.0001(5)$ & $0.0003(4)$ \\
O1A & $0.047(3)$ & $0.053(3)$ & $0.048(3)$ & $-0.015(2)$ & $-0.005(2)$ & $0.000(2)$ \\
C1A & $0.024(2)$ & $0.030(2)$ & $0.021(2)$ & $-0.003(2)$ & $0.003(2)$ & $0.0002(17)$ \\
C2A & $0.035(3)$ & $0.035(2)$ & $0.025(2)$ & $-0.013(2)$ & $0.005(2)$ & $-0.006(2)$ \\
C3A & $0.037(3)$ & $0.050(3)$ & $0.033(3)$ & $-0.018(3)$ & $0.004(3)$ & $-0.010(3)$ \\
C4A & $0.026(3)$ & $0.056(3)$ & $0.028(3)$ & $-0.005(3)$ & $-0.001(2)$ & $-0.004(3)$
\end{tabular}




\begin{tabular}{|c|c|c|c|c|c|c|}
\hline $\mathrm{C} 5 \mathrm{~A}$ & $0.026(3)$ & $0.038(3)$ & $0.024(2)$ & $0.003(2)$ & $-0.003(2)$ & $0.001(2)$ \\
\hline C6A & $0.027(3)$ & $0.047(3)$ & $0.032(3)$ & $0.000(3)$ & 0.007 (2) & $-0.013(2)$ \\
\hline C7A & $0.041(3)$ & $0.045(3)$ & $0.031(3)$ & $0.007(3)$ & $0.007(3)$ & $-0.004(2)$ \\
\hline $\mathrm{C} 8 \mathrm{~A}$ & $0.046(4)$ & $0.039(3)$ & $0.032(3)$ & $-0.007(3)$ & $0.005(3)$ & $-0.013(2)$ \\
\hline C9A & $0.038(3)$ & $0.048(3)$ & $0.022(2)$ & $-0.001(3)$ & $-0.001(2)$ & $-0.002(2)$ \\
\hline $\mathrm{C} 10 \mathrm{~A}$ & $0.035(3)$ & $0.043(3)$ & $0.029(3)$ & $-0.002(3)$ & $0.008(2)$ & $-0.002(2)$ \\
\hline $\mathrm{C} 11 \mathrm{~A}$ & $0.058(4)$ & $0.035(3)$ & $0.037(3)$ & $-0.011(3)$ & $0.006(3)$ & $0.002(2)$ \\
\hline $\mathrm{C} 12 \mathrm{~A}$ & $0.070(6)$ & $0.042(3)$ & $0.077(5)$ & $-0.022(4)$ & $-0.010(5)$ & $0.006(3)$ \\
\hline $\mathrm{C} 15 \mathrm{~A}$ & $0.023(2)$ & $0.025(2)$ & $0.020(2)$ & $0.002(2)$ & $0.004(2)$ & $0.0043(17)$ \\
\hline C16A & $0.027(3)$ & $0.030(2)$ & $0.023(2)$ & $-0.001(2)$ & $0.000(2)$ & $0.0026(18)$ \\
\hline $\mathrm{C} 17 \mathrm{~A}$ & $0.037(3)$ & $0.037(3)$ & $0.028(3)$ & $0.001(3)$ & $0.007(3)$ & $-0.003(2)$ \\
\hline $\mathrm{C} 18 \mathrm{~A}$ & $0.027(3)$ & $0.044(3)$ & $0.042(3)$ & $0.004(3)$ & $0.009(3)$ & -0.003 \\
\hline $\mathrm{C} 19 \mathrm{~A}$ & $0.024(3)$ & $0.038(3)$ & $0.031(3)$ & $-0.001(2)$ & $-0.003(2)$ & $-0.001(2)$ \\
\hline $\mathrm{C} 20 \mathrm{~A}$ & $0.077(6)$ & $0.040(3)$ & $0.050(4)$ & -0.005 (4) & $0.010(4)$ & $0.019(3)$ \\
\hline $\mathrm{C} 21 \mathrm{~A}$ & $0.071(5)$ & 0.067 (4) & $0.037(3)$ & $-0.012(4)$ & $-0.001(4)$ & $0.027(3)$ \\
\hline $\mathrm{C} 22 \mathrm{~A}$ & $0.106(8)$ & $0.063(4)$ & $0.036(4)$ & $-0.018(5)$ & $0.019(5)$ & $0.015(3)$ \\
\hline $\mathrm{C} 23 \mathrm{~A}$ & $0.061(5)$ & $0.065(5)$ & $0.074(5)$ & $-0.021(4)$ & $0.026(5)$ & $0.026(4)$ \\
\hline $\mathrm{C} 24 \mathrm{~A}$ & $0.078(6)$ & $0.048(4)$ & $0.066(5)$ & $-0.027(4)$ & $0.005(5)$ & $0.016(4)$ \\
\hline $\mathrm{C} 25 \mathrm{~A}$ & $0.037(3)$ & $0.044(3)$ & $0.037(3)$ & $-0.001(3)$ & -0.010 & $-0.003(2)$ \\
\hline $\mathrm{C} 26 \mathrm{~A}$ & $0.072(6)$ & $0.087(6)$ & $0.044(4)$ & $-0.020(5)$ & $-0.020(4)$ & $0.000(4)$ \\
\hline $\mathrm{C} 34 \mathrm{~A}$ & $0.035(3)$ & $0.026(2)$ & $0.018(2)$ & $-0.006(2)$ & $0.000(2)$ & $-0.0009(17)$ \\
\hline C35A & $0.046(3)$ & $0.029(2)$ & $0.032(3)$ & -0.001 & $0.001(3)$ & $0.001(2)$ \\
\hline C36A & $0.074(5)$ & $0.028(2)$ & $0.036(3)$ & $-0.006(3)$ & $-0.004(4)$ & $0.002(2)$ \\
\hline $\mathrm{C} 37 \mathrm{~A}$ & $0.075(5)$ & $0.043(3)$ & $0.031(3)$ & $-0.032(4)$ & $0.000(3)$ & $0.007(2)$ \\
\hline C38A & $0.048(4)$ & $0.060(4)$ & $0.034(3)$ & $-0.024(3)$ & $0.002(3)$ & $0.000(3)$ \\
\hline C39A & $0.031(3)$ & $0.039(3)$ & 0.034 (3) & $-0.008(3)$ & $-0.001(2)$ & $-0.004(2)$ \\
\hline Fe1B & $0.0244(4)$ & $0.0691(6)$ & $0.0460(5)$ & $-0.0012(4)$ & $0.0028(4)$ & $0.0336(5)$ \\
\hline $\mathrm{Fe} 2 \mathrm{~B}$ & $0.0566(6)$ & $0.0410(4)$ & $0.0242(4)$ & $-0.0133(4)$ & $0.0019(4)$ & $-0.0041(3)$ \\
\hline S1B & $0.0309(7)$ & $0.0297(6)$ & $0.0515(9)$ & $-0.0042(6)$ & $-0.0037(7)$ & $0.0023(6)$ \\
\hline P1B & $0.0249(7)$ & $0.0259(5)$ & $0.0320(7)$ & $-0.0017(5)$ & $0.0000(6)$ & $0.0063(5)$ \\
\hline O1B & $0.063(5)$ & $0.275(12)$ & $0.100(6)$ & $0.064(7)$ & $0.015(4)$ & $0.114(7)$ \\
\hline $\mathrm{C} 1 \mathrm{~B}$ & $0.024(3)$ & 0.035 (3) & $0.033(3)$ & $0.001(2)$ & $0.006(2)$ & $0.010(2)$ \\
\hline $\mathrm{C} 2 \mathrm{~B}$ & $0.027(3)$ & $0.037(3)$ & 0.049 (3) & $-0.001(2)$ & $-0.003(3)$ & 0.017 (2) \\
\hline C3B & $0.024(3)$ & $0.052(3)$ & $0.048(3)$ & $0.000(3)$ & $0.000(3)$ & $0.025(3)$ \\
\hline C4B & $0.030(3)$ & $0.072(4)$ & $0.037(3)$ & $-0.002(3)$ & $-0.002(3)$ & $0.018(3)$ \\
\hline C5B & $0.034(3)$ & $0.046(3)$ & $0.034(3)$ & $-0.004(3)$ & $0.003(3)$ & $0.007(2)$ \\
\hline C6B & $0.023(3)$ & $0.119(7)$ & $0.088(6)$ & -0.004 (4) & 0.009 (4) & $0.073(6)$ \\
\hline C7B & $0.046(5)$ & $0.100(6)$ & $0.090(7)$ & $-0.021(5)$ & $0.002(5)$ & $0.061(5)$ \\
\hline $\mathrm{C} 8 \mathrm{~B}$ & $0.031(4)$ & $0.128(8)$ & $0.070(6)$ & $-0.004(5)$ & $0.002(4)$ & $0.074(6)$ \\
\hline C9B & $0.056(5)$ & $0.120(8)$ & $0.053(5)$ & $-0.001(6)$ & $0.010(4)$ & $0.044(5)$ \\
\hline $\mathrm{C} 10 \mathrm{~B}$ & $0.042(5)$ & $0.133(9)$ & $0.075(6)$ & $0.015(6)$ & $0.019(5)$ & $0.052(6)$ \\
\hline C11B & $0.041(4)$ & $0.027(2)$ & $0.068(4)$ & $0.003(3)$ & $-0.013(3)$ & $0.006(3)$ \\
\hline $\mathrm{C} 12 \mathrm{~B}$ & $0.066(6)$ & $0.047(4)$ & $0.101(7)$ & $0.017(4)$ & $-0.025(5)$ & $-0.005(4)$ \\
\hline C15B & $0.031(3)$ & $0.028(2)$ & $0.032(3)$ & $-0.005(2)$ & $-0.001(2)$ & $0.0065(19)$ \\
\hline $\mathrm{C} 16 \mathrm{~B}$ & $0.048(4)$ & $0.039(3)$ & $0.044(3)$ & -0.009 & $-0.008(3)$ & $0.019(3)$ \\
\hline C17B & $0.054(4)$ & $0.049(3)$ & $0.038(3)$ & $-0.015(3)$ & $0.004(3)$ & $0.012(3)$ \\
\hline $\mathrm{C} 18 \mathrm{~B}$ & $0.041(3)$ & $0.044(3)$ & $0.036(3)$ & $-0.015(3)$ & $0.008(3)$ & $-0.003(3)$ \\
\hline C19B & $0.033(3)$ & $0.040(3)$ & $0.028(3)$ & $-0.005(3)$ & $0.004(2)$ & -0.005 (2) \\
\hline
\end{tabular}




$\begin{array}{lllllll}\mathrm{C} 20 \mathrm{~B} & 0.056(4) & 0.040(3) & 0.064(5) & -0.011(3) & -0.001(4) & -0.011(3) \\ \mathrm{C} 21 \mathrm{~B} & 0.137(10) & 0.057(5) & 0.066(6) & 0.007(6) & -0.056(6) & -0.030(4) \\ \mathrm{C} 22 \mathrm{~B} & 0.26(2) & 0.063(5) & 0.044(5) & -0.046(9) & 0.052(8) & -0.030(4) \\ \mathrm{C} 23 \mathrm{~B} & 0.112(9) & 0.096(7) & 0.101(8) & -0.040(7) & 0.067(8) & -0.070(7) \\ \mathrm{C} 24 \mathrm{~B} & 0.063(5) & 0.066(4) & 0.055(4) & 0.000(4) & -0.001(4) & -0.027(4) \\ \mathrm{C} 25 \mathrm{~B} & 0.075(6) & 0.076(5) & 0.105(8) & -0.020(5) & -0.041(6) & 0.061(6) \\ \mathrm{C} 26 \mathrm{~B} & 0.090(7) & 0.061(5) & 0.088(6) & -0.018(5) & -0.053(6) & 0.033(4) \\ \mathrm{C} 34 \mathrm{~B} & 0.031(3) & 0.030(2) & 0.028(3) & 0.002(2) & -0.002(2) & 0.007(2) \\ \mathrm{C} 35 \mathrm{~B} & 0.041(3) & 0.032(2) & 0.046(3) & -0.003(3) & 0.001(3) & 0.004(2) \\ \mathrm{C} 36 \mathrm{~B} & 0.057(4) & 0.033(3) & 0.056(4) & 0.003(3) & 0.002(3) & 0.000(3) \\ \mathrm{C} 37 \mathrm{~B} & 0.057(4) & 0.042(3) & 0.048(4) & 0.011(3) & 0.007(3) & -0.004(3) \\ \mathrm{C} 38 \mathrm{~B} & 0.047(4) & 0.051(3) & 0.045(3) & 0.001(3) & 0.015(3) & 0.001(3) \\ \mathrm{C} 39 \mathrm{~B} & 0.036(3) & 0.036(3) & 0.031(3) & -0.002(3) & 0.001(3) & 0.005(2) \\ & & & & & \end{array}$

Geometric parameters $\left(A,{ }^{\circ}\right)$

\begin{tabular}{|c|c|c|c|}
\hline $\mathrm{Fe} 1 \mathrm{~A}-\mathrm{C} 1 \mathrm{~A}$ & $2.023(5)$ & $\mathrm{Fe} 1 \mathrm{~B}-\mathrm{C} 1 \mathrm{~B}$ & $2.016(5)$ \\
\hline $\mathrm{Fe} 1 \mathrm{~A}-\mathrm{C} 2 \mathrm{~A}$ & $2.041(5)$ & $\mathrm{Fe} 1 \mathrm{~B}-\mathrm{C} 2 \mathrm{~B}$ & $2.050(7)$ \\
\hline $\mathrm{Fe} 1 \mathrm{~A}-\mathrm{C} 3 \mathrm{~A}$ & $2.035(6)$ & $\mathrm{Fe} 1 \mathrm{~B}-\mathrm{C} 3 \mathrm{~B}$ & $2.058(6)$ \\
\hline $\mathrm{Fe} 1 \mathrm{~A}-\mathrm{C} 4 \mathrm{~A}$ & $2.053(6)$ & $\mathrm{Fe} 1 \mathrm{~B}-\mathrm{C} 4 \mathrm{~B}$ & $2.021(7)$ \\
\hline $\mathrm{Fe} 1 \mathrm{~A}-\mathrm{C} 5 \mathrm{~A}$ & $2.032(5)$ & $\mathrm{Fe} 1 \mathrm{~B}-\mathrm{C} 5 \mathrm{~B}$ & $2.022(6)$ \\
\hline $\mathrm{Fe} 1 \mathrm{~A}-\mathrm{C} 6 \mathrm{~A}$ & $2.048(6)$ & $\mathrm{Fe} 1 \mathrm{~B}-\mathrm{C} 6 \mathrm{~B}$ & $2.035(7)$ \\
\hline $\mathrm{Fe} 1 \mathrm{~A}-\mathrm{C} 7 \mathrm{~A}$ & $2.050(6)$ & $\mathrm{Fe} 1 \mathrm{~B}-\mathrm{C} 7 \mathrm{~B}$ & $2.043(8)$ \\
\hline $\mathrm{Fe} 1 \mathrm{~A}-\mathrm{C} 8 \mathrm{~A}$ & $2.036(6)$ & $\mathrm{Fe} 1 \mathrm{~B}-\mathrm{C} 8 \mathrm{~B}$ & $2.031(7)$ \\
\hline $\mathrm{Fe} 1 \mathrm{~A}-\mathrm{C} 9 \mathrm{~A}$ & $2.039(5)$ & $\mathrm{Fe} 1 \mathrm{~B}-\mathrm{C} 9 \mathrm{~B}$ & $2.058(8)$ \\
\hline $\mathrm{Fe} 1 \mathrm{~A}-\mathrm{C} 10 \mathrm{~A}$ & $2.035(6)$ & $\mathrm{Fe} 1 \mathrm{~B}-\mathrm{C} 10 \mathrm{~B}$ & $2.071(9)$ \\
\hline $\mathrm{Fe} 2 \mathrm{~A}-\mathrm{C} 15 \mathrm{~A}$ & $2.026(5)$ & $\mathrm{Fe} 2 \mathrm{~B}-\mathrm{C} 15 \mathrm{~B}$ & $2.017(6)$ \\
\hline $\mathrm{Fe} 2 \mathrm{~A}-\mathrm{C} 16 \mathrm{~A}$ & $2.029(5)$ & $\mathrm{Fe} 2 \mathrm{~B}-\mathrm{C} 16 \mathrm{~B}$ & $2.034(7)$ \\
\hline $\mathrm{Fe} 2 \mathrm{~A}-\mathrm{C} 17 \mathrm{~A}$ & $2.034(5)$ & $\mathrm{Fe} 2 \mathrm{~B}-\mathrm{C} 17 \mathrm{~B}$ & $2.034(7)$ \\
\hline $\mathrm{Fe} 2 \mathrm{~A}-\mathrm{C} 18 \mathrm{~A}$ & $2.057(6)$ & $\mathrm{Fe} 2 \mathrm{~B}-\mathrm{C} 18 \mathrm{~B}$ & $2.051(6)$ \\
\hline $\mathrm{Fe} 2 \mathrm{~A}-\mathrm{C} 19 \mathrm{~A}$ & $2.039(6)$ & $\mathrm{Fe} 2 \mathrm{~B}-\mathrm{C} 19 \mathrm{~B}$ & $2.035(6)$ \\
\hline $\mathrm{Fe} 2 \mathrm{~A}-\mathrm{C} 20 \mathrm{~A}$ & $2.067(7)$ & $\mathrm{Fe} 2 \mathrm{~B}-\mathrm{C} 20 \mathrm{~B}$ & $2.038(6)$ \\
\hline $\mathrm{Fe} 2 \mathrm{~A}-\mathrm{C} 21 \mathrm{~A}$ & $2.034(7)$ & $\mathrm{Fe} 2 \mathrm{~B}-\mathrm{C} 21 \mathrm{~B}$ & $2.039(7)$ \\
\hline $\mathrm{Fe} 2 \mathrm{~A}-\mathrm{C} 22 \mathrm{~A}$ & $2.029(7)$ & $\mathrm{Fe} 2 \mathrm{~B}-\mathrm{C} 22 \mathrm{~B}$ & $2.024(8)$ \\
\hline $\mathrm{Fe} 2 \mathrm{~A}-\mathrm{C} 23 \mathrm{~A}$ & $2.028(7)$ & $\mathrm{Fe} 2 \mathrm{~B}-\mathrm{C} 23 \mathrm{~B}$ & $2.025(10)$ \\
\hline $\mathrm{Fe} 2 \mathrm{~A}-\mathrm{C} 24 \mathrm{~A}$ & $2.052(7)$ & $\mathrm{Fe} 2 \mathrm{~B}-\mathrm{C} 24 \mathrm{~B}$ & $2.036(8)$ \\
\hline $\mathrm{S} 1 \mathrm{~A}-\mathrm{P} 1 \mathrm{~A}$ & $1.9552(18)$ & $\mathrm{S} 1 \mathrm{~B}-\mathrm{P} 1 \mathrm{~B}$ & 1.959 (2) \\
\hline $\mathrm{P} 1 \mathrm{~A}-\mathrm{C} 1 \mathrm{~A}$ & $1.797(5)$ & $\mathrm{P} 1 \mathrm{~B}-\mathrm{C} 1 \mathrm{~B}$ & $1.798(6)$ \\
\hline $\mathrm{P} 1 \mathrm{~A}-\mathrm{C} 15 \mathrm{~A}$ & $1.793(5)$ & $\mathrm{P} 1 \mathrm{~B}-\mathrm{C} 15 \mathrm{~B}$ & $1.791(5)$ \\
\hline $\mathrm{P} 1 \mathrm{~A}-\mathrm{C} 34 \mathrm{~A}$ & $1.822(5)$ & $\mathrm{P} 1 \mathrm{~B}-\mathrm{C} 34 \mathrm{~B}$ & $1.830(6)$ \\
\hline $\mathrm{O} 1 \mathrm{~A}-\mathrm{H} 1 \mathrm{~A}$ & 0.8400 & $\mathrm{O} 1 \mathrm{~B}-\mathrm{H} 1 \mathrm{~B}$ & 0.8400 \\
\hline $\mathrm{O} 1 \mathrm{~A}-\mathrm{C} 25 \mathrm{~A}$ & $1.460(7)$ & $\mathrm{O} 1 \mathrm{~B}-\mathrm{C} 25 \mathrm{~B}$ & $1.458(14)$ \\
\hline $\mathrm{C} 1 \mathrm{~A}-\mathrm{C} 2 \mathrm{~A}$ & $1.454(7)$ & $\mathrm{C} 1 \mathrm{~B}-\mathrm{C} 2 \mathrm{~B}$ & $1.439(8)$ \\
\hline $\mathrm{C} 1 \mathrm{~A}-\mathrm{C} 5 \mathrm{~A}$ & $1.436(7)$ & $\mathrm{C} 1 \mathrm{~B}-\mathrm{C} 5 \mathrm{~B}$ & $1.440(8)$ \\
\hline $\mathrm{C} 2 \mathrm{~A}-\mathrm{C} 3 \mathrm{~A}$ & $1.432(8)$ & $\mathrm{C} 2 \mathrm{~B}-\mathrm{C} 3 \mathrm{~B}$ & $1.424(9)$ \\
\hline $\mathrm{C} 2 \mathrm{~A}-\mathrm{C} 11 \mathrm{~A}$ & $1.456(8)$ & $\mathrm{C} 2 \mathrm{~B}-\mathrm{C} 11 \mathrm{~B}$ & $1.469(9)$ \\
\hline $\mathrm{C} 3 \mathrm{~A}-\mathrm{H} 3 \mathrm{~A}$ & 0.9500 & $\mathrm{C} 3 \mathrm{~B}-\mathrm{H} 3 \mathrm{~B}$ & 0.9500 \\
\hline $\mathrm{C} 3 \mathrm{~A}-\mathrm{C} 4 \mathrm{~A}$ & $1.418(9)$ & $\mathrm{C} 3 \mathrm{~B}-\mathrm{C} 4 \mathrm{~B}$ & $1.417(10)$ \\
\hline
\end{tabular}




\begin{tabular}{|c|c|c|c|}
\hline $\mathrm{C} 4 \mathrm{~A}-\mathrm{H} 4 \mathrm{~A}$ & 0.9500 & $\mathrm{C} 4 \mathrm{~B}-\mathrm{H} 4 \mathrm{~B}$ & 0.9500 \\
\hline $\mathrm{C} 4 \mathrm{~A}-\mathrm{C} 5 \mathrm{~A}$ & $1.413(8)$ & $\mathrm{C} 4 \mathrm{~B}-\mathrm{C} 5 \mathrm{~B}$ & $1.408(9)$ \\
\hline $\mathrm{C} 5 \mathrm{~A}-\mathrm{H} 5 \mathrm{~A}$ & 0.9500 & $\mathrm{C} 5 \mathrm{~B}-\mathrm{H} 5 \mathrm{~B}$ & 0.9500 \\
\hline C6A-H6A & 0.9500 & $\mathrm{C} 6 \mathrm{~B}-\mathrm{H} 6 \mathrm{~B}$ & 0.9500 \\
\hline $\mathrm{C} 6 \mathrm{~A}-\mathrm{C} 7 \mathrm{~A}$ & $1.425(8)$ & $\mathrm{C} 6 \mathrm{~B}-\mathrm{C} 7 \mathrm{~B}$ & $1.398(13)$ \\
\hline $\mathrm{C} 6 \mathrm{~A}-\mathrm{C} 10 \mathrm{~A}$ & $1.412(8)$ & $\mathrm{C} 6 \mathrm{~B}-\mathrm{C} 10 \mathrm{~B}$ & $1.441(15)$ \\
\hline C7A-H7A & 0.9500 & $\mathrm{C} 7 \mathrm{~B}-\mathrm{H} 7 \mathrm{~B}$ & 0.9500 \\
\hline $\mathrm{C} 7 \mathrm{~A}-\mathrm{C} 8 \mathrm{~A}$ & $1.417(9)$ & $\mathrm{C} 7 \mathrm{~B}-\mathrm{C} 8 \mathrm{~B}$ & $1.440(13)$ \\
\hline $\mathrm{C} 8 \mathrm{~A}-\mathrm{H} 8 \mathrm{~A}$ & 0.9500 & $\mathrm{C} 8 \mathrm{~B}-\mathrm{H} 8 \mathrm{~B}$ & 0.9500 \\
\hline $\mathrm{C} 8 \mathrm{~A}-\mathrm{C} 9 \mathrm{~A}$ & $1.420(8)$ & $\mathrm{C} 8 \mathrm{~B}-\mathrm{C} 9 \mathrm{~B}$ & $1.396(14)$ \\
\hline C9A-H9A & 0.9500 & C9B-H9B & 0.9500 \\
\hline C9A-C10A & $1.424(8)$ & $\mathrm{C} 9 \mathrm{~B}-\mathrm{C} 10 \mathrm{~B}$ & $1.431(12)$ \\
\hline $\mathrm{C} 10 \mathrm{~A}-\mathrm{H} 10 \mathrm{~A}$ & 0.9500 & $\mathrm{C} 10 \mathrm{~B}-\mathrm{H} 10 \mathrm{~B}$ & 0.9500 \\
\hline $\mathrm{C} 11 \mathrm{~A}-\mathrm{H} 11 \mathrm{~A}$ & 0.9500 & C11B-H11B & 0.9500 \\
\hline $\mathrm{C} 11 \mathrm{~A}-\mathrm{C} 12 \mathrm{~A}$ & $1.305(9)$ & $\mathrm{C} 11 \mathrm{~B}-\mathrm{C} 12 \mathrm{~B}$ & $1.329(10)$ \\
\hline $\mathrm{C} 12 \mathrm{~A}-\mathrm{H} 12 \mathrm{~A}$ & 0.9500 & $\mathrm{C} 12 \mathrm{~B}-\mathrm{H} 12 \mathrm{C}$ & 0.9500 \\
\hline $\mathrm{C} 12 \mathrm{~A}-\mathrm{H} 12 \mathrm{~B}$ & 0.9500 & $\mathrm{C} 12 \mathrm{~B}-\mathrm{H} 12 \mathrm{D}$ & 0.9500 \\
\hline $\mathrm{C} 15 \mathrm{~A}-\mathrm{C} 16 \mathrm{~A}$ & $1.444(7)$ & $\mathrm{C} 15 \mathrm{~B}-\mathrm{C} 16 \mathrm{~B}$ & $1.449(8)$ \\
\hline $\mathrm{C} 15 \mathrm{~A}-\mathrm{C} 19 \mathrm{~A}$ & $1.443(7)$ & $\mathrm{C} 15 \mathrm{~B}-\mathrm{C} 19 \mathrm{~B}$ & $1.430(8)$ \\
\hline $\mathrm{C} 16 \mathrm{~A}-\mathrm{C} 17 \mathrm{~A}$ & $1.420(8)$ & $\mathrm{C} 16 \mathrm{~B}-\mathrm{C} 17 \mathrm{~B}$ & $1.421(9)$ \\
\hline $\mathrm{C} 16 \mathrm{~A}-\mathrm{C} 25 \mathrm{~A}$ & $1.513(8)$ & $\mathrm{C} 16 \mathrm{~B}-\mathrm{C} 25 \mathrm{~B}$ & $1.507(11)$ \\
\hline $\mathrm{C} 17 \mathrm{~A}-\mathrm{H} 17 \mathrm{~A}$ & 0.9500 & $\mathrm{C} 17 \mathrm{~B}-\mathrm{H} 17 \mathrm{~B}$ & 0.9500 \\
\hline $\mathrm{C} 17 \mathrm{~A}-\mathrm{C} 18 \mathrm{~A}$ & $1.404(9)$ & $\mathrm{C} 17 \mathrm{~B}-\mathrm{C} 18 \mathrm{~B}$ & $1.398(10)$ \\
\hline $\mathrm{C} 18 \mathrm{~A}-\mathrm{H} 18 \mathrm{~A}$ & 0.9500 & $\mathrm{C} 18 \mathrm{~B}-\mathrm{H} 18 \mathrm{~B}$ & 0.9500 \\
\hline $\mathrm{C} 18 \mathrm{~A}-\mathrm{C} 19 \mathrm{~A}$ & $1.427(8)$ & $\mathrm{C} 18 \mathrm{~B}-\mathrm{C} 19 \mathrm{~B}$ & $1.420(8)$ \\
\hline C19A-H19A & 0.9500 & C19B-H19B & 0.9500 \\
\hline $\mathrm{C} 20 \mathrm{~A}-\mathrm{H} 20 \mathrm{~A}$ & 0.9500 & $\mathrm{C} 20 \mathrm{~B}-\mathrm{H} 20 \mathrm{~B}$ & 0.9500 \\
\hline $\mathrm{C} 20 \mathrm{~A}-\mathrm{C} 21 \mathrm{~A}$ & $1.425(11)$ & $\mathrm{C} 20 \mathrm{~B}-\mathrm{C} 21 \mathrm{~B}$ & $1.443(12)$ \\
\hline $\mathrm{C} 20 \mathrm{~A}-\mathrm{C} 24 \mathrm{~A}$ & $1.425(12)$ & $\mathrm{C} 20 \mathrm{~B}-\mathrm{C} 24 \mathrm{~B}$ & $1.383(11)$ \\
\hline $\mathrm{C} 21 \mathrm{~A}-\mathrm{H} 21 \mathrm{~A}$ & 0.9500 & $\mathrm{C} 21 \mathrm{~B}-\mathrm{H} 21 \mathrm{~B}$ & 0.9500 \\
\hline $\mathrm{C} 21 \mathrm{~A}-\mathrm{C} 22 \mathrm{~A}$ & $1.385(12)$ & $\mathrm{C} 21 \mathrm{~B}-\mathrm{C} 22 \mathrm{~B}$ & $1.395(18)$ \\
\hline $\mathrm{C} 22 \mathrm{~A}-\mathrm{H} 22 \mathrm{~A}$ & 0.9500 & $\mathrm{C} 22 \mathrm{~B}-\mathrm{H} 22 \mathrm{~B}$ & 0.9500 \\
\hline $\mathrm{C} 22 \mathrm{~A}-\mathrm{C} 23 \mathrm{~A}$ & $1.411(13)$ & $\mathrm{C} 22 \mathrm{~B}-\mathrm{C} 23 \mathrm{~B}$ & $1.364(19)$ \\
\hline $\mathrm{C} 23 \mathrm{~A}-\mathrm{H} 23 \mathrm{~A}$ & 0.9500 & $\mathrm{C} 23 \mathrm{~B}-\mathrm{H} 23 \mathrm{~B}$ & 0.9500 \\
\hline $\mathrm{C} 23 \mathrm{~A}-\mathrm{C} 24 \mathrm{~A}$ & 1.404 (12) & $\mathrm{C} 23 \mathrm{~B}-\mathrm{C} 24 \mathrm{~B}$ & $1.385(14)$ \\
\hline $\mathrm{C} 24 \mathrm{~A}-\mathrm{H} 24 \mathrm{~A}$ & 0.9500 & $\mathrm{C} 24 \mathrm{~B}-\mathrm{H} 24 \mathrm{~B}$ & 0.9500 \\
\hline $\mathrm{C} 25 \mathrm{~A}-\mathrm{H} 25 \mathrm{~A}$ & 1.0000 & $\mathrm{C} 25 \mathrm{~B}-\mathrm{H} 25 \mathrm{~B}$ & 1.0000 \\
\hline $\mathrm{C} 25 \mathrm{~A}-\mathrm{C} 26 \mathrm{~A}$ & $1.463(9)$ & $\mathrm{C} 25 \mathrm{~B}-\mathrm{C} 26 \mathrm{~B}$ & $1.540(12)$ \\
\hline $\mathrm{C} 26 \mathrm{~A}-\mathrm{H} 26 \mathrm{~A}$ & 0.9800 & $\mathrm{C} 26 \mathrm{~B}-\mathrm{H} 26 \mathrm{D}$ & 0.9800 \\
\hline $\mathrm{C} 26 \mathrm{~A}-\mathrm{H} 26 \mathrm{~B}$ & 0.9800 & $\mathrm{C} 26 \mathrm{~B}-\mathrm{H} 26 \mathrm{E}$ & 0.9800 \\
\hline $\mathrm{C} 26 \mathrm{~A}-\mathrm{H} 26 \mathrm{C}$ & 0.9800 & $\mathrm{C} 26 \mathrm{~B}-\mathrm{H} 26 \mathrm{~F}$ & 0.9800 \\
\hline $\mathrm{C} 34 \mathrm{~A}-\mathrm{C} 35 \mathrm{~A}$ & $1.399(8)$ & $\mathrm{C} 34 \mathrm{~B}-\mathrm{C} 35 \mathrm{~B}$ & $1.392(8)$ \\
\hline $\mathrm{C} 34 \mathrm{~A}-\mathrm{C} 39 \mathrm{~A}$ & $1.397(8)$ & $\mathrm{C} 34 \mathrm{~B}-\mathrm{C} 39 \mathrm{~B}$ & $1.379(8)$ \\
\hline $\mathrm{C} 35 \mathrm{~A}-\mathrm{H} 35 \mathrm{~A}$ & 0.9500 & $\mathrm{C} 35 \mathrm{~B}-\mathrm{H} 35 \mathrm{~B}$ & 0.9500 \\
\hline $\mathrm{C} 35 \mathrm{~A}-\mathrm{C} 36 \mathrm{~A}$ & $1.399(8)$ & $\mathrm{C} 35 \mathrm{~B}-\mathrm{C} 36 \mathrm{~B}$ & $1.384(9)$ \\
\hline $\mathrm{C} 36 \mathrm{~A}-\mathrm{H} 36 \mathrm{~A}$ & 0.9500 & $\mathrm{C} 36 \mathrm{~B}-\mathrm{H} 36 \mathrm{~B}$ & 0.9500 \\
\hline $\mathrm{C} 36 \mathrm{~A}-\mathrm{C} 37 \mathrm{~A}$ & $1.382(11)$ & $\mathrm{C} 36 \mathrm{~B}-\mathrm{C} 37 \mathrm{~B}$ & $1.379(10)$ \\
\hline $\mathrm{C} 37 \mathrm{~A}-\mathrm{H} 37 \mathrm{~A}$ & 0.9500 & $\mathrm{C} 37 \mathrm{~B}-\mathrm{H} 37 \mathrm{~B}$ & 0.9500 \\
\hline
\end{tabular}




\begin{tabular}{|c|c|}
\hline $\mathrm{C} 37 \mathrm{~A}-\mathrm{C} 38 \mathrm{~A}$ & $1.378(10)$ \\
\hline C38A-H38A & 0.9500 \\
\hline $\mathrm{C} 38 \mathrm{~A}-\mathrm{C} 39 \mathrm{~A}$ & $1.379(8)$ \\
\hline C39A-H39A & 0.9500 \\
\hline $\mathrm{C} 1 \mathrm{~A}-\mathrm{Fe} 1 \mathrm{~A}-\mathrm{C} 2 \mathrm{~A}$ & $41.91(19)$ \\
\hline $\mathrm{C} 1 \mathrm{~A}-\mathrm{Fe} 1 \mathrm{~A}-\mathrm{C} 3 \mathrm{~A}$ & $69.4(2)$ \\
\hline $\mathrm{C} 1 \mathrm{~A}-\mathrm{Fe} 1 \mathrm{~A}-\mathrm{C} 4 \mathrm{~A}$ & $69.1(2)$ \\
\hline $\mathrm{C} 1 \mathrm{~A}-\mathrm{Fe} 1 \mathrm{~A}-\mathrm{C} 5 \mathrm{~A}$ & $41.5(2)$ \\
\hline $\mathrm{C} 1 \mathrm{~A}-\mathrm{Fe} 1 \mathrm{~A}-\mathrm{C} 6 \mathrm{~A}$ & $108.4(2)$ \\
\hline $\mathrm{C} 1 \mathrm{~A}-\mathrm{Fe} 1 \mathrm{~A}-\mathrm{C} 7 \mathrm{~A}$ & $124.0(2)$ \\
\hline $\mathrm{C} 1 \mathrm{~A}-\mathrm{Fe} 1 \mathrm{~A}-\mathrm{C} 8 \mathrm{~A}$ & $159.8(2)$ \\
\hline $\mathrm{C} 1 \mathrm{~A}-\mathrm{Fe} 1 \mathrm{~A}-\mathrm{C} 9 \mathrm{~A}$ & $158.5(2)$ \\
\hline $\mathrm{C} 1 \mathrm{~A}-\mathrm{Fe} 1 \mathrm{~A}-\mathrm{C} 10 \mathrm{~A}$ & $123.0(2)$ \\
\hline $\mathrm{C} 2 \mathrm{~A}-\mathrm{Fe} 1 \mathrm{~A}-\mathrm{C} 4 \mathrm{~A}$ & $69.2(3)$ \\
\hline $\mathrm{C} 2 \mathrm{~A}-\mathrm{Fe} 1 \mathrm{~A}-\mathrm{C} 6 \mathrm{~A}$ & $121.3(2)$ \\
\hline $\mathrm{C} 2 \mathrm{~A}-\mathrm{Fe} 1 \mathrm{~A}-\mathrm{C} 7 \mathrm{~A}$ & $105.4(2)$ \\
\hline $\mathrm{C} 3 \mathrm{~A}-\mathrm{Fe} 1 \mathrm{~A}-\mathrm{C} 2 \mathrm{~A}$ & $41.1(2)$ \\
\hline $\mathrm{C} 3 \mathrm{~A}-\mathrm{Fe} 1 \mathrm{~A}-\mathrm{C} 4 \mathrm{~A}$ & $40.6(3)$ \\
\hline $\mathrm{C} 3 \mathrm{~A}-\mathrm{Fe} 1 \mathrm{~A}-\mathrm{C} 6 \mathrm{~A}$ & $156.2(3)$ \\
\hline $\mathrm{C} 3 \mathrm{~A}-\mathrm{Fe} 1 \mathrm{~A}-\mathrm{C} 7 \mathrm{~A}$ & $119.5(3)$ \\
\hline $\mathrm{C} 3 \mathrm{~A}-\mathrm{Fe} 1 \mathrm{~A}-\mathrm{C} 8 \mathrm{~A}$ & $105.0(3)$ \\
\hline $\mathrm{C} 3 \mathrm{~A}-\mathrm{Fe} 1 \mathrm{~A}-\mathrm{C} 9 \mathrm{~A}$ & $122.3(2)$ \\
\hline $\mathrm{C} 3 \mathrm{~A}-\mathrm{Fe} 1 \mathrm{~A}-\mathrm{C} 10 \mathrm{~A}$ & $160.4(3)$ \\
\hline $\mathrm{C} 5 \mathrm{~A}-\mathrm{Fe} 1 \mathrm{~A}-\mathrm{C} 2 \mathrm{~A}$ & $69.9(2)$ \\
\hline $\mathrm{C} 5 \mathrm{~A}-\mathrm{Fe} 1 \mathrm{~A}-\mathrm{C} 3 \mathrm{~A}$ & $68.6(2)$ \\
\hline $\mathrm{C} 5 \mathrm{~A}-\mathrm{Fe} 1 \mathrm{~A}-\mathrm{C} 4 \mathrm{~A}$ & $40.5(2)$ \\
\hline $\mathrm{C} 5 \mathrm{~A}-\mathrm{Fe} 1 \mathrm{~A}-\mathrm{C} 6 \mathrm{~A}$ & $126.4(2)$ \\
\hline $\mathrm{C} 5 \mathrm{~A}-\mathrm{Fe} 1 \mathrm{~A}-\mathrm{C} 7 \mathrm{~A}$ & $162.5(2)$ \\
\hline $\mathrm{C} 5 \mathrm{~A}-\mathrm{Fe} 1 \mathrm{~A}-\mathrm{C} 8 \mathrm{~A}$ & $156.3(2)$ \\
\hline $\mathrm{C} 5 \mathrm{~A}-\mathrm{Fe} 1 \mathrm{~A}-\mathrm{C} 9 \mathrm{~A}$ & $122.0(2)$ \\
\hline $\mathrm{C} 5 \mathrm{~A}-\mathrm{Fe} 1 \mathrm{~A}-\mathrm{C} 10 \mathrm{~A}$ & $109.5(2)$ \\
\hline $\mathrm{C} 6 \mathrm{~A}-\mathrm{Fe} 1 \mathrm{~A}-\mathrm{C} 4 \mathrm{~A}$ & $162.6(3)$ \\
\hline $\mathrm{C} 6 \mathrm{~A}-\mathrm{Fe} 1 \mathrm{~A}-\mathrm{C} 7 \mathrm{~A}$ & $40.7(2)$ \\
\hline $\mathrm{C} 7 \mathrm{~A}-\mathrm{Fe} 1 \mathrm{~A}-\mathrm{C} 4 \mathrm{~A}$ & $155.0(3)$ \\
\hline $\mathrm{C} 8 \mathrm{~A}-\mathrm{Fe} 1 \mathrm{~A}-\mathrm{C} 2 \mathrm{~A}$ & $121.3(2)$ \\
\hline $\mathrm{C} 8 \mathrm{~A}-\mathrm{Fe} 1 \mathrm{~A}-\mathrm{C} 4 \mathrm{~A}$ & $120.0(3)$ \\
\hline $\mathrm{C} 8 \mathrm{~A}-\mathrm{Fe} 1 \mathrm{~A}-\mathrm{C} 6 \mathrm{~A}$ & $68.4(3)$ \\
\hline $\mathrm{C} 8 \mathrm{~A}-\mathrm{Fe} 1 \mathrm{~A}-\mathrm{C} 7 \mathrm{~A}$ & $40.6(3)$ \\
\hline $\mathrm{C} 8 \mathrm{~A}-\mathrm{Fe} 1 \mathrm{~A}-\mathrm{C} 9 \mathrm{~A}$ & $40.8(2)$ \\
\hline $\mathrm{C} 9 \mathrm{~A}-\mathrm{Fe} 1 \mathrm{~A}-\mathrm{C} 2 \mathrm{~A}$ & $158.3(2)$ \\
\hline $\mathrm{C} 9 \mathrm{~A}-\mathrm{Fe} 1 \mathrm{~A}-\mathrm{C} 4 \mathrm{~A}$ & $107.0(2)$ \\
\hline $\mathrm{C} 9 \mathrm{~A}-\mathrm{Fe} 1 \mathrm{~A}-\mathrm{C} 6 \mathrm{~A}$ & $68.5(2)$ \\
\hline $\mathrm{C} 9 \mathrm{~A}-\mathrm{Fe} 1 \mathrm{~A}-\mathrm{C} 7 \mathrm{~A}$ & $68.6(2)$ \\
\hline $\mathrm{C} 10 \mathrm{~A}-\mathrm{Fe} 1 \mathrm{~A}-\mathrm{C} 2 \mathrm{~A}$ & $158.1(2)$ \\
\hline $\mathrm{C} 10 \mathrm{~A}-\mathrm{Fe} 1 \mathrm{~A}-\mathrm{C} 4 \mathrm{~A}$ & $125.3(2)$ \\
\hline $\mathrm{C} 10 \mathrm{~A}-\mathrm{Fe} 1 \mathrm{~A}-\mathrm{C} 6 \mathrm{~A}$ & $40.5(2)$ \\
\hline & \\
\hline
\end{tabular}

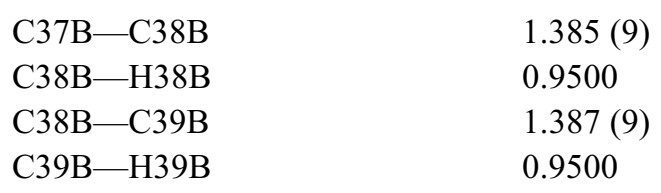




\begin{tabular}{|c|c|}
\hline $\mathrm{C} 10 \mathrm{~A}-\mathrm{Fe} 1 \mathrm{~A}-\mathrm{C} 8 \mathrm{~A}$ & $68.5(2)$ \\
\hline $\mathrm{C} 10 \mathrm{~A}-\mathrm{Fe} 1 \mathrm{~A}-\mathrm{C} 9 \mathrm{~A}$ & $40.9(2)$ \\
\hline $\mathrm{C} 15 \mathrm{~A}-\mathrm{Fe} 2 \mathrm{~A}-\mathrm{C} 16 \mathrm{~A}$ & $41.73(19)$ \\
\hline $\mathrm{C} 15 \mathrm{~A}-\mathrm{Fe} 2 \mathrm{~A}-\mathrm{C} 17 \mathrm{~A}$ & $69.0(2)$ \\
\hline $\mathrm{C} 15 \mathrm{~A}-\mathrm{Fe} 2 \mathrm{~A}-\mathrm{C} 18 \mathrm{~A}$ & $69.1(2)$ \\
\hline $\mathrm{C} 15 \mathrm{~A}-\mathrm{Fe} 2 \mathrm{~A}-\mathrm{C} 19 \mathrm{~A}$ & $41.6(2)$ \\
\hline $\mathrm{C} 15 \mathrm{~A}-\mathrm{Fe} 2 \mathrm{~A}-\mathrm{C} 20 \mathrm{~A}$ & $110.4(3)$ \\
\hline $\mathrm{C} 15 \mathrm{~A}-\mathrm{Fe} 2 \mathrm{~A}-\mathrm{C} 21 \mathrm{~A}$ & $126.2(3)$ \\
\hline $\mathrm{C} 15 \mathrm{~A}-\mathrm{Fe} 2 \mathrm{~A}-\mathrm{C} 22 \mathrm{~A}$ & $160.2(3)$ \\
\hline $\mathrm{C} 15 \mathrm{~A}-\mathrm{Fe} 2 \mathrm{~A}-\mathrm{C} 23 \mathrm{~A}$ & $158.6(3)$ \\
\hline $\mathrm{C} 15 \mathrm{~A}-\mathrm{Fe} 2 \mathrm{~A}-\mathrm{C} 24 \mathrm{~A}$ & $124.4(3)$ \\
\hline $\mathrm{C} 16 \mathrm{~A}-\mathrm{Fe} 2 \mathrm{~A}-\mathrm{C} 17 \mathrm{~A}$ & $40.9(2)$ \\
\hline $\mathrm{C} 16 \mathrm{~A}-\mathrm{Fe} 2 \mathrm{~A}-\mathrm{C} 18 \mathrm{~A}$ & $68.9(2)$ \\
\hline $\mathrm{C} 16 \mathrm{~A}-\mathrm{Fe} 2 \mathrm{~A}-\mathrm{C} 19 \mathrm{~A}$ & $69.9(2)$ \\
\hline $\mathrm{C} 16 \mathrm{~A}-\mathrm{Fe} 2 \mathrm{~A}-\mathrm{C} 20 \mathrm{~A}$ & $124.3(3)$ \\
\hline $\mathrm{C} 16 \mathrm{~A}-\mathrm{Fe} 2 \mathrm{~A}-\mathrm{C} 21 \mathrm{~A}$ & $108.1(3)$ \\
\hline $\mathrm{C} 16 \mathrm{~A}-\mathrm{Fe} 2 \mathrm{~A}-\mathrm{C} 22 \mathrm{~A}$ & $121.5(3)$ \\
\hline $\mathrm{C} 16 \mathrm{~A}-\mathrm{Fe} 2 \mathrm{~A}-\mathrm{C} 24 \mathrm{~A}$ & $160.9(3)$ \\
\hline $\mathrm{C} 17 \mathrm{~A}-\mathrm{Fe} 2 \mathrm{~A}-\mathrm{C} 18 \mathrm{~A}$ & $40.1(2)$ \\
\hline $\mathrm{C} 17 \mathrm{~A}-\mathrm{Fe} 2 \mathrm{~A}-\mathrm{C} 19 \mathrm{~A}$ & $68.4(2)$ \\
\hline $\mathrm{C} 17 \mathrm{~A}-\mathrm{Fe} 2 \mathrm{~A}-\mathrm{C} 20 \mathrm{~A}$ & $158.8(3)$ \\
\hline $\mathrm{C} 17 \mathrm{~A}-\mathrm{Fe} 2 \mathrm{~A}-\mathrm{C} 24 \mathrm{~A}$ & $157.5(3)$ \\
\hline $\mathrm{C} 18 \mathrm{~A}-\mathrm{Fe} 2 \mathrm{~A}-\mathrm{C} 20 \mathrm{~A}$ & $160.9(3)$ \\
\hline $\mathrm{C} 19 \mathrm{~A}-\mathrm{Fe} 2 \mathrm{~A}-\mathrm{C} 18 \mathrm{~A}$ & $40.8(2)$ \\
\hline $\mathrm{C} 19 \mathrm{~A}-\mathrm{Fe} 2 \mathrm{~A}-\mathrm{C} 20 \mathrm{~A}$ & $126.2(3)$ \\
\hline $\mathrm{C} 19 \mathrm{~A}-\mathrm{Fe} 2 \mathrm{~A}-\mathrm{C} 24 \mathrm{~A}$ & $108.4(3)$ \\
\hline $\mathrm{C} 21 \mathrm{~A}-\mathrm{Fe} 2 \mathrm{~A}-\mathrm{C} 17 \mathrm{~A}$ & $121.4(3)$ \\
\hline $\mathrm{C} 21 \mathrm{~A}-\mathrm{Fe} 2 \mathrm{~A}-\mathrm{C} 18 \mathrm{~A}$ & $155.0(3)$ \\
\hline $\mathrm{C} 21 \mathrm{~A}-\mathrm{Fe} 2 \mathrm{~A}-\mathrm{C} 19 \mathrm{~A}$ & $163.5(3)$ \\
\hline $\mathrm{C} 21 \mathrm{~A}-\mathrm{Fe} 2 \mathrm{~A}-\mathrm{C} 20 \mathrm{~A}$ & $40.6(3)$ \\
\hline $\mathrm{C} 21 \mathrm{~A}-\mathrm{Fe} 2 \mathrm{~A}-\mathrm{C} 24 \mathrm{~A}$ & $67.9(3)$ \\
\hline $\mathrm{C} 22 \mathrm{~A}-\mathrm{Fe} 2 \mathrm{~A}-\mathrm{C} 17 \mathrm{~A}$ & $104.9(3)$ \\
\hline $\mathrm{C} 22 \mathrm{~A}-\mathrm{Fe} 2 \mathrm{~A}-\mathrm{C} 18 \mathrm{~A}$ & $119.0(3)$ \\
\hline $\mathrm{C} 22 \mathrm{~A}-\mathrm{Fe} 2 \mathrm{~A}-\mathrm{C} 19 \mathrm{~A}$ & $155.4(4)$ \\
\hline $\mathrm{C} 22 \mathrm{~A}-\mathrm{Fe} 2 \mathrm{~A}-\mathrm{C} 20 \mathrm{~A}$ & $68.1(3)$ \\
\hline $\mathrm{C} 22 \mathrm{~A}-\mathrm{Fe} 2 \mathrm{~A}-\mathrm{C} 21 \mathrm{~A}$ & $39.9(4)$ \\
\hline $\mathrm{C} 22 \mathrm{~A}-\mathrm{Fe} 2 \mathrm{~A}-\mathrm{C} 24 \mathrm{~A}$ & $68.2(3)$ \\
\hline $\mathrm{C} 23 \mathrm{~A}-\mathrm{Fe} 2 \mathrm{~A}-\mathrm{C} 16 \mathrm{~A}$ & $157.3(3)$ \\
\hline $\mathrm{C} 23 \mathrm{~A}-\mathrm{Fe} 2 \mathrm{~A}-\mathrm{C} 17 \mathrm{~A}$ & $120.8(3)$ \\
\hline $\mathrm{C} 23 \mathrm{~A}-\mathrm{Fe} 2 \mathrm{~A}-\mathrm{C} 18 \mathrm{~A}$ & $105.2(3)$ \\
\hline $\mathrm{C} 23 \mathrm{~A}-\mathrm{Fe} 2 \mathrm{~A}-\mathrm{C} 19 \mathrm{~A}$ & $120.8(3)$ \\
\hline $\mathrm{C} 23 \mathrm{~A}-\mathrm{Fe} 2 \mathrm{~A}-\mathrm{C} 20 \mathrm{~A}$ & $67.9(3)$ \\
\hline $\mathrm{C} 23 \mathrm{~A}-\mathrm{Fe} 2 \mathrm{~A}-\mathrm{C} 21 \mathrm{~A}$ & $67.5(4)$ \\
\hline $\mathrm{C} 23 \mathrm{~A}-\mathrm{Fe} 2 \mathrm{~A}-\mathrm{C} 22 \mathrm{~A}$ & $40.7(4)$ \\
\hline $\mathrm{C} 23 \mathrm{~A}-\mathrm{Fe} 2 \mathrm{~A}-\mathrm{C} 24 \mathrm{~A}$ & $40.3(3)$ \\
\hline $\mathrm{C} 24 \mathrm{~A}-\mathrm{Fe} 2 \mathrm{~A}-\mathrm{C} 18 \mathrm{~A}$ & $122.9(3)$ \\
\hline $\mathrm{C} 24 \mathrm{~A}-\mathrm{Fe} 2 \mathrm{~A}-\mathrm{C} 20 \mathrm{~A}$ & $40.5(3)$ \\
\hline $\mathrm{C} 1 \mathrm{~A}-\mathrm{P} 1 \mathrm{~A}-\mathrm{S} 1 \mathrm{~A}$ & $117.33(18$ \\
\hline
\end{tabular}

\begin{tabular}{|c|c|}
\hline $\mathrm{C} 9 \mathrm{~B}-\mathrm{Fe} 1 \mathrm{~B}-\mathrm{C} 3 \mathrm{~B}$ & $122.1(3)$ \\
\hline $\mathrm{C} 9 \mathrm{~B}-\mathrm{Fe} 1 \mathrm{~B}-\mathrm{C} 10 \mathrm{~B}$ & $40.5(4)$ \\
\hline $\mathrm{C} 15 \mathrm{~B}-\mathrm{Fe} 2 \mathrm{~B}-\mathrm{C} 16 \mathrm{~B}$ & $41.9(2)$ \\
\hline $\mathrm{C} 15 \mathrm{~B}-\mathrm{Fe} 2 \mathrm{~B}-\mathrm{C} 17 \mathrm{~B}$ & $69.1(2)$ \\
\hline $\mathrm{C} 15 \mathrm{~B}-\mathrm{Fe} 2 \mathrm{~B}-\mathrm{C} 18 \mathrm{~B}$ & $69.2(2)$ \\
\hline $\mathrm{C} 15 \mathrm{~B}-\mathrm{Fe} 2 \mathrm{~B}-\mathrm{C} 19 \mathrm{~B}$ & $41.3(2)$ \\
\hline $\mathrm{C} 15 \mathrm{~B}-\mathrm{Fe} 2 \mathrm{~B}-\mathrm{C} 20 \mathrm{~B}$ & $109.9(3)$ \\
\hline $\mathrm{C} 15 \mathrm{~B}-\mathrm{Fe} 2 \mathrm{~B}-\mathrm{C} 21 \mathrm{~B}$ & $128.1(4)$ \\
\hline $\mathrm{C} 15 \mathrm{~B}-\mathrm{Fe} 2 \mathrm{~B}-\mathrm{C} 22 \mathrm{~B}$ & $164.5(6)$ \\
\hline $\mathrm{C} 15 \mathrm{~B}-\mathrm{Fe} 2 \mathrm{~B}-\mathrm{C} 23 \mathrm{~B}$ & $155.4(5)$ \\
\hline $\mathrm{C} 15 \mathrm{~B}-\mathrm{Fe} 2 \mathrm{~B}-\mathrm{C} 24 \mathrm{~B}$ & $122.2(3)$ \\
\hline $\mathrm{C} 16 \mathrm{~B}-\mathrm{Fe} 2 \mathrm{~B}-\mathrm{C} 18 \mathrm{~B}$ & $69.0(3)$ \\
\hline $\mathrm{C} 16 \mathrm{~B}-\mathrm{Fe} 2 \mathrm{~B}-\mathrm{C} 19 \mathrm{~B}$ & $69.5(3)$ \\
\hline $\mathrm{C} 16 \mathrm{~B}-\mathrm{Fe} 2 \mathrm{~B}-\mathrm{C} 20 \mathrm{~B}$ & $123.0(3)$ \\
\hline $\mathrm{C} 16 \mathrm{~B}-\mathrm{Fe} 2 \mathrm{~B}-\mathrm{C} 21 \mathrm{~B}$ & $108.6(4)$ \\
\hline $\mathrm{C} 16 \mathrm{~B}-\mathrm{Fe} 2 \mathrm{~B}-\mathrm{C} 24 \mathrm{~B}$ & $158.1(3)$ \\
\hline $\mathrm{C} 17 \mathrm{~B}-\mathrm{Fe} 2 \mathrm{~B}-\mathrm{C} 16 \mathrm{~B}$ & $40.9(3)$ \\
\hline $\mathrm{C} 17 \mathrm{~B}-\mathrm{Fe} 2 \mathrm{~B}-\mathrm{C} 18 \mathrm{~B}$ & $40.0(3)$ \\
\hline $\mathrm{C} 17 \mathrm{~B}-\mathrm{Fe} 2 \mathrm{~B}-\mathrm{C} 19 \mathrm{~B}$ & $68.0(3)$ \\
\hline $\mathrm{C} 17 \mathrm{~B}-\mathrm{Fe} 2 \mathrm{~B}-\mathrm{C} 20 \mathrm{~B}$ & $157.5(3)$ \\
\hline $\mathrm{C} 17 \mathrm{~B}-\mathrm{Fe} 2 \mathrm{~B}-\mathrm{C} 21 \mathrm{~B}$ & $120.4(3)$ \\
\hline $\mathrm{C} 17 \mathrm{~B}-\mathrm{Fe} 2 \mathrm{~B}-\mathrm{C} 24 \mathrm{~B}$ & $160.2(3)$ \\
\hline $\mathrm{C} 19 \mathrm{~B}-\mathrm{Fe} 2 \mathrm{~B}-\mathrm{C} 18 \mathrm{~B}$ & $40.7(2)$ \\
\hline $\mathrm{C} 19 \mathrm{~B}-\mathrm{Fe} 2 \mathrm{~B}-\mathrm{C} 20 \mathrm{~B}$ & $127.0(3)$ \\
\hline $\mathrm{C} 19 \mathrm{~B}-\mathrm{Fe} 2 \mathrm{~B}-\mathrm{C} 21 \mathrm{~B}$ & $165.9(4)$ \\
\hline $\mathrm{C} 19 \mathrm{~B}-\mathrm{Fe} 2 \mathrm{~B}-\mathrm{C} 24 \mathrm{~B}$ & $108.6(3)$ \\
\hline $\mathrm{C} 20 \mathrm{~B}-\mathrm{Fe} 2 \mathrm{~B}-\mathrm{C} 18 \mathrm{~B}$ & $162.2(3)$ \\
\hline $\mathrm{C} 20 \mathrm{~B}-\mathrm{Fe} 2 \mathrm{~B}-\mathrm{C} 21 \mathrm{~B}$ & $41.5(3)$ \\
\hline $\mathrm{C} 21 \mathrm{~B}-\mathrm{Fe} 2 \mathrm{~B}-\mathrm{C} 18 \mathrm{~B}$ & $152.9(4)$ \\
\hline $\mathrm{C} 22 \mathrm{~B}-\mathrm{Fe} 2 \mathrm{~B}-\mathrm{C} 16 \mathrm{~B}$ & $125.3(5)$ \\
\hline $\mathrm{C} 22 \mathrm{~B}-\mathrm{Fe} 2 \mathrm{~B}-\mathrm{C} 17 \mathrm{~B}$ & $106.7(4)$ \\
\hline $\mathrm{C} 22 \mathrm{~B}-\mathrm{Fe} 2 \mathrm{~B}-\mathrm{C} 18 \mathrm{~B}$ & $117.8(4)$ \\
\hline $\mathrm{C} 22 \mathrm{~B}-\mathrm{Fe} 2 \mathrm{~B}-\mathrm{C} 19 \mathrm{~B}$ & $152.4(5)$ \\
\hline $\mathrm{C} 22 \mathrm{~B}-\mathrm{Fe} 2 \mathrm{~B}-\mathrm{C} 20 \mathrm{~B}$ & $68.0(4)$ \\
\hline $\mathrm{C} 22 \mathrm{~B}-\mathrm{Fe} 2 \mathrm{~B}-\mathrm{C} 21 \mathrm{~B}$ & $40.2(5)$ \\
\hline $\mathrm{C} 22 \mathrm{~B}-\mathrm{Fe} 2 \mathrm{~B}-\mathrm{C} 23 \mathrm{~B}$ & $39.4(6)$ \\
\hline $\mathrm{C} 22 \mathrm{~B}-\mathrm{Fe} 2 \mathrm{~B}-\mathrm{C} 24 \mathrm{~B}$ & $66.7(5)$ \\
\hline $\mathrm{C} 23 \mathrm{~B}-\mathrm{Fe} 2 \mathrm{~B}-\mathrm{C} 16 \mathrm{~B}$ & $160.6(5)$ \\
\hline $\mathrm{C} 23 \mathrm{~B}-\mathrm{Fe} 2 \mathrm{~B}-\mathrm{C} 17 \mathrm{~B}$ & $123.3(4)$ \\
\hline $\mathrm{C} 23 \mathrm{~B}-\mathrm{Fe} 2 \mathrm{~B}-\mathrm{C} 18 \mathrm{~B}$ & $105.6(3)$ \\
\hline $\mathrm{C} 23 \mathrm{~B}-\mathrm{Fe} 2 \mathrm{~B}-\mathrm{C} 19 \mathrm{~B}$ & $119.3(4)$ \\
\hline $\mathrm{C} 23 \mathrm{~B}-\mathrm{Fe} 2 \mathrm{~B}-\mathrm{C} 20 \mathrm{~B}$ & $67.4(4)$ \\
\hline $\mathrm{C} 23 \mathrm{~B}-\mathrm{Fe} 2 \mathrm{~B}-\mathrm{C} 21 \mathrm{~B}$ & $67.2(5)$ \\
\hline $\mathrm{C} 23 \mathrm{~B}-\mathrm{Fe} 2 \mathrm{~B}-\mathrm{C} 24 \mathrm{~B}$ & $39.9(4)$ \\
\hline $\mathrm{C} 24 \mathrm{~B}-\mathrm{Fe} 2 \mathrm{~B}-\mathrm{C} 18 \mathrm{~B}$ & $124.7(3)$ \\
\hline $\mathrm{C} 24 \mathrm{~B}-\mathrm{Fe} 2 \mathrm{~B}-\mathrm{C} 20 \mathrm{~B}$ & $39.7(3)$ \\
\hline $\mathrm{C} 24 \mathrm{~B}-\mathrm{Fe} 2 \mathrm{~B}-\mathrm{C} 21 \mathrm{~B}$ & $67.6(4)$ \\
\hline $\mathrm{C} 1 \mathrm{~B}-\mathrm{P} 1 \mathrm{~B}-\mathrm{S} 1 \mathrm{~B}$ & $117.24(19)$ \\
\hline
\end{tabular}




\begin{tabular}{|c|c|}
\hline $\mathrm{C} 1 \mathrm{~A}-\mathrm{P} 1 \mathrm{~A}-\mathrm{C} 34 \mathrm{~A}$ & $104.9(2)$ \\
\hline $\mathrm{C} 15 \mathrm{~A}-\mathrm{P} 1 \mathrm{~A}-\mathrm{S} 1 \mathrm{~A}$ & $116.44(17)$ \\
\hline $\mathrm{C} 15 \mathrm{~A}-\mathrm{P} 1 \mathrm{~A}-\mathrm{C} 1 \mathrm{~A}$ & $102.8(2)$ \\
\hline $\mathrm{C} 15 \mathrm{~A}-\mathrm{P} 1 \mathrm{~A}-\mathrm{C} 34 \mathrm{~A}$ & $103.5(2)$ \\
\hline $\mathrm{C} 34 \mathrm{~A}-\mathrm{P} 1 \mathrm{~A}-\mathrm{S} 1 \mathrm{~A}$ & $110.5(2)$ \\
\hline $\mathrm{C} 25 \mathrm{~A}-\mathrm{O} 1 \mathrm{~A}-\mathrm{H} 1 \mathrm{~A}$ & 109.5 \\
\hline $\mathrm{P} 1 \mathrm{~A}-\mathrm{C} 1 \mathrm{~A}-\mathrm{Fe} 1 \mathrm{~A}$ & $128.6(3)$ \\
\hline $\mathrm{C} 2 \mathrm{~A}-\mathrm{C} 1 \mathrm{~A}-\mathrm{Fe} 1 \mathrm{~A}$ & $69.7(3)$ \\
\hline $\mathrm{C} 2 \mathrm{~A}-\mathrm{C} 1 \mathrm{~A}-\mathrm{P} 1 \mathrm{~A}$ & $128.3(4)$ \\
\hline $\mathrm{C} 5 \mathrm{~A}-\mathrm{C} 1 \mathrm{~A}-\mathrm{Fe} 1 \mathrm{~A}$ & $69.6(3)$ \\
\hline $\mathrm{C} 5 \mathrm{~A}-\mathrm{C} 1 \mathrm{~A}-\mathrm{P} 1 \mathrm{~A}$ & $124.0(4)$ \\
\hline $\mathrm{C} 5 \mathrm{~A}-\mathrm{C} 1 \mathrm{~A}-\mathrm{C} 2 \mathrm{~A}$ & $107.6(5)$ \\
\hline $\mathrm{C} 1 \mathrm{~A}-\mathrm{C} 2 \mathrm{~A}-\mathrm{Fe} 1 \mathrm{~A}$ & $68.4(3)$ \\
\hline $\mathrm{C} 1 \mathrm{~A}-\mathrm{C} 2 \mathrm{~A}-\mathrm{C} 11 \mathrm{~A}$ & $127.9(5)$ \\
\hline $\mathrm{C} 3 \mathrm{~A}-\mathrm{C} 2 \mathrm{~A}-\mathrm{Fe} 1 \mathrm{~A}$ & $69.2(3)$ \\
\hline $\mathrm{C} 3 \mathrm{~A}-\mathrm{C} 2 \mathrm{~A}-\mathrm{C} 1 \mathrm{~A}$ & $106.4(5)$ \\
\hline $\mathrm{C} 3 \mathrm{~A}-\mathrm{C} 2 \mathrm{~A}-\mathrm{C} 11 \mathrm{~A}$ & $125.4(5)$ \\
\hline $\mathrm{C} 11 \mathrm{~A}-\mathrm{C} 2 \mathrm{~A}-\mathrm{Fe} 1 \mathrm{~A}$ & $122.5(5)$ \\
\hline $\mathrm{Fe} 1 \mathrm{~A}-\mathrm{C} 3 \mathrm{~A}-\mathrm{H} 3 \mathrm{~A}$ & 126.2 \\
\hline $\mathrm{C} 2 \mathrm{~A}-\mathrm{C} 3 \mathrm{~A}-\mathrm{Fe} 1 \mathrm{~A}$ & $69.7(3)$ \\
\hline $\mathrm{C} 2 \mathrm{~A}-\mathrm{C} 3 \mathrm{~A}-\mathrm{H} 3 \mathrm{~A}$ & 125.3 \\
\hline $\mathrm{C} 4 \mathrm{~A}-\mathrm{C} 3 \mathrm{~A}-\mathrm{Fe} 1 \mathrm{~A}$ & $70.4(3)$ \\
\hline $\mathrm{C} 4 \mathrm{~A}-\mathrm{C} 3 \mathrm{~A}-\mathrm{C} 2 \mathrm{~A}$ & $109.3(5)$ \\
\hline $\mathrm{C} 4 \mathrm{~A}-\mathrm{C} 3 \mathrm{~A}-\mathrm{H} 3 \mathrm{~A}$ & 125.3 \\
\hline $\mathrm{Fe} 1 \mathrm{~A}-\mathrm{C} 4 \mathrm{~A}-\mathrm{H} 4 \mathrm{~A}$ & 127.6 \\
\hline $\mathrm{C} 3 \mathrm{~A}-\mathrm{C} 4 \mathrm{~A}-\mathrm{Fe} 1 \mathrm{~A}$ & $69.0(4)$ \\
\hline $\mathrm{C} 3 \mathrm{~A}-\mathrm{C} 4 \mathrm{~A}-\mathrm{H} 4 \mathrm{~A}$ & 125.9 \\
\hline $\mathrm{C} 5 \mathrm{~A}-\mathrm{C} 4 \mathrm{~A}-\mathrm{Fe} 1 \mathrm{~A}$ & $69.0(3)$ \\
\hline $\mathrm{C} 5 \mathrm{~A}-\mathrm{C} 4 \mathrm{~A}-\mathrm{C} 3 \mathrm{~A}$ & $108.2(5)$ \\
\hline $\mathrm{C} 5 \mathrm{~A}-\mathrm{C} 4 \mathrm{~A}-\mathrm{H} 4 \mathrm{~A}$ & 125.9 \\
\hline $\mathrm{Fe} 1 \mathrm{~A}-\mathrm{C} 5 \mathrm{~A}-\mathrm{H} 5 \mathrm{~A}$ & 126.4 \\
\hline $\mathrm{C} 1 \mathrm{~A}-\mathrm{C} 5 \mathrm{~A}-\mathrm{Fe} 1 \mathrm{~A}$ & $68.9(3)$ \\
\hline $\mathrm{C} 1 \mathrm{~A}-\mathrm{C} 5 \mathrm{~A}-\mathrm{H} 5 \mathrm{~A}$ & 125.8 \\
\hline $\mathrm{C} 4 \mathrm{~A}-\mathrm{C} 5 \mathrm{~A}-\mathrm{Fe} 1 \mathrm{~A}$ & $70.5(3)$ \\
\hline $\mathrm{C} 4 \mathrm{~A}-\mathrm{C} 5 \mathrm{~A}-\mathrm{C} 1 \mathrm{~A}$ & $108.5(5)$ \\
\hline $\mathrm{C} 4 \mathrm{~A}-\mathrm{C} 5 \mathrm{~A}-\mathrm{H} 5 \mathrm{~A}$ & 125.8 \\
\hline $\mathrm{Fe} 1 \mathrm{~A}-\mathrm{C} 6 \mathrm{~A}-\mathrm{H} 6 \mathrm{~A}$ & 126.5 \\
\hline $\mathrm{C} 7 \mathrm{~A}-\mathrm{C} 6 \mathrm{~A}-\mathrm{Fe} 1 \mathrm{~A}$ & $69.8(3)$ \\
\hline $\mathrm{C} 7 \mathrm{~A}-\mathrm{C} 6 \mathrm{~A}-\mathrm{H} 6 \mathrm{~A}$ & 126.0 \\
\hline $\mathrm{C} 10 \mathrm{~A}-\mathrm{C} 6 \mathrm{~A}-\mathrm{Fe} 1 \mathrm{~A}$ & $69.3(3)$ \\
\hline $\mathrm{C} 10 \mathrm{~A}-\mathrm{C} 6 \mathrm{~A}-\mathrm{H} 6 \mathrm{~A}$ & 126.0 \\
\hline $\mathrm{C} 10 \mathrm{~A}-\mathrm{C} 6 \mathrm{~A}-\mathrm{C} 7 \mathrm{~A}$ & $108.0(5)$ \\
\hline $\mathrm{Fe} 1 \mathrm{~A}-\mathrm{C} 7 \mathrm{~A}-\mathrm{H} 7 \mathrm{~A}$ & 126.7 \\
\hline $\mathrm{C} 6 \mathrm{~A}-\mathrm{C} 7 \mathrm{~A}-\mathrm{Fe} 1 \mathrm{~A}$ & $69.5(3)$ \\
\hline $\mathrm{C} 6 \mathrm{~A}-\mathrm{C} 7 \mathrm{~A}-\mathrm{H} 7 \mathrm{~A}$ & 126.2 \\
\hline $\mathrm{C} 8 \mathrm{~A}-\mathrm{C} 7 \mathrm{~A}-\mathrm{Fe} 1 \mathrm{~A}$ & $69.2(4)$ \\
\hline $\mathrm{C} 8 \mathrm{~A}-\mathrm{C} 7 \mathrm{~A}-\mathrm{C} 6 \mathrm{~A}$ & $107.7(5)$ \\
\hline & \\
\hline
\end{tabular}

\begin{tabular}{|c|c|}
\hline $\mathrm{C} 1 \mathrm{~B}-\mathrm{P} 1 \mathrm{~B}-\mathrm{C} 34 \mathrm{~B}$ & $103.4(3)$ \\
\hline $\mathrm{C} 15 \mathrm{~B}-\mathrm{P} 1 \mathrm{~B}-\mathrm{S} 1 \mathrm{~B}$ & $116.4(2)$ \\
\hline $\mathrm{C} 15 \mathrm{~B}-\mathrm{P} 1 \mathrm{~B}-\mathrm{C} 1 \mathrm{~B}$ & $104.2(3)$ \\
\hline $\mathrm{C} 15 \mathrm{~B}-\mathrm{P} 1 \mathrm{~B}-\mathrm{C} 34 \mathrm{~B}$ & $101.9(2)$ \\
\hline $\mathrm{C} 34 \mathrm{~B}-\mathrm{P} 1 \mathrm{~B}-\mathrm{S} 1 \mathrm{~B}$ & $111.9(2)$ \\
\hline $\mathrm{C} 25 \mathrm{~B}-\mathrm{O} 1 \mathrm{~B}-\mathrm{H} 1 \mathrm{~B}$ & 109.5 \\
\hline $\mathrm{P} 1 \mathrm{~B}-\mathrm{C} 1 \mathrm{~B}-\mathrm{Fe} 1 \mathrm{~B}$ & $126.8(3)$ \\
\hline $\mathrm{C} 2 \mathrm{~B}-\mathrm{C} 1 \mathrm{~B}-\mathrm{Fe} 1 \mathrm{~B}$ & $70.6(3)$ \\
\hline $\mathrm{C} 2 \mathrm{~B}-\mathrm{C} 1 \mathrm{~B}-\mathrm{P} 1 \mathrm{~B}$ & $129.1(5)$ \\
\hline $\mathrm{C} 2 \mathrm{~B}-\mathrm{C} 1 \mathrm{~B}-\mathrm{C} 5 \mathrm{~B}$ & $107.8(5)$ \\
\hline $\mathrm{C} 5 \mathrm{~B}-\mathrm{C} 1 \mathrm{~B}-\mathrm{Fe} 1 \mathrm{~B}$ & $69.3(3)$ \\
\hline $\mathrm{C} 5 \mathrm{~B}-\mathrm{C} 1 \mathrm{~B}-\mathrm{P} 1 \mathrm{~B}$ & $123.1(4)$ \\
\hline $\mathrm{C} 1 \mathrm{~B}-\mathrm{C} 2 \mathrm{~B}-\mathrm{Fe} 1 \mathrm{~B}$ & $68.0(3)$ \\
\hline $\mathrm{C} 1 \mathrm{~B}-\mathrm{C} 2 \mathrm{~B}-\mathrm{C} 11 \mathrm{~B}$ & $127.5(5)$ \\
\hline $\mathrm{C} 3 \mathrm{~B}-\mathrm{C} 2 \mathrm{~B}-\mathrm{Fe} 1 \mathrm{~B}$ & $70.0(4)$ \\
\hline $\mathrm{C} 3 \mathrm{~B}-\mathrm{C} 2 \mathrm{~B}-\mathrm{C} 1 \mathrm{~B}$ & $107.7(6)$ \\
\hline $\mathrm{C} 3 \mathrm{~B}-\mathrm{C} 2 \mathrm{~B}-\mathrm{C} 11 \mathrm{~B}$ & $124.9(6)$ \\
\hline $\mathrm{C} 11 \mathrm{~B}-\mathrm{C} 2 \mathrm{~B}-\mathrm{Fe} 1 \mathrm{~B}$ & $127.4(5)$ \\
\hline $\mathrm{Fe} 1 \mathrm{~B}-\mathrm{C} 3 \mathrm{~B}-\mathrm{H} 3 \mathrm{~B}$ & 127.7 \\
\hline $\mathrm{C} 2 \mathrm{~B}-\mathrm{C} 3 \mathrm{~B}-\mathrm{Fe} 1 \mathrm{~B}$ & $69.4(4)$ \\
\hline $\mathrm{C} 2 \mathrm{~B}-\mathrm{C} 3 \mathrm{~B}-\mathrm{H} 3 \mathrm{~B}$ & 126.2 \\
\hline $\mathrm{C} 4 \mathrm{~B}-\mathrm{C} 3 \mathrm{~B}-\mathrm{Fe} 1 \mathrm{~B}$ & $68.3(4)$ \\
\hline $\mathrm{C} 4 \mathrm{~B}-\mathrm{C} 3 \mathrm{~B}-\mathrm{C} 2 \mathrm{~B}$ & $107.6(5)$ \\
\hline $\mathrm{C} 4 \mathrm{~B}-\mathrm{C} 3 \mathrm{~B}-\mathrm{H} 3 \mathrm{~B}$ & 126.2 \\
\hline $\mathrm{Fe} 1 \mathrm{~B}-\mathrm{C} 4 \mathrm{~B}-\mathrm{H} 4 \mathrm{~B}$ & 125.8 \\
\hline $\mathrm{C} 3 \mathrm{~B}-\mathrm{C} 4 \mathrm{~B}-\mathrm{Fe} 1 \mathrm{~B}$ & $71.1(4)$ \\
\hline $\mathrm{C} 3 \mathrm{~B}-\mathrm{C} 4 \mathrm{~B}-\mathrm{H} 4 \mathrm{~B}$ & 125.1 \\
\hline $\mathrm{C} 5 \mathrm{~B}-\mathrm{C} 4 \mathrm{~B}-\mathrm{Fe} 1 \mathrm{~B}$ & $69.7(4)$ \\
\hline $\mathrm{C} 5 \mathrm{~B}-\mathrm{C} 4 \mathrm{~B}-\mathrm{C} 3 \mathrm{~B}$ & $109.9(6)$ \\
\hline $\mathrm{C} 5 \mathrm{~B}-\mathrm{C} 4 \mathrm{~B}-\mathrm{H} 4 \mathrm{~B}$ & 125.1 \\
\hline $\mathrm{Fe} 1 \mathrm{~B}-\mathrm{C} 5 \mathrm{~B}-\mathrm{H} 5 \mathrm{~B}$ & 126.6 \\
\hline $\mathrm{C} 1 \mathrm{~B}-\mathrm{C} 5 \mathrm{~B}-\mathrm{Fe} 1 \mathrm{~B}$ & $68.9(3)$ \\
\hline $\mathrm{C} 1 \mathrm{~B}-\mathrm{C} 5 \mathrm{~B}-\mathrm{H} 5 \mathrm{~B}$ & 126.5 \\
\hline $\mathrm{C} 4 \mathrm{~B}-\mathrm{C} 5 \mathrm{~B}-\mathrm{Fe} 1 \mathrm{~B}$ & $69.6(4)$ \\
\hline $\mathrm{C} 4 \mathrm{~B}-\mathrm{C} 5 \mathrm{~B}-\mathrm{C} 1 \mathrm{~B}$ & $107.0(6)$ \\
\hline $\mathrm{C} 4 \mathrm{~B}-\mathrm{C} 5 \mathrm{~B}-\mathrm{H} 5 \mathrm{~B}$ & 126.5 \\
\hline $\mathrm{Fe} 1 \mathrm{~B}-\mathrm{C} 6 \mathrm{~B}-\mathrm{H} 6 \mathrm{~B}$ & 125.7 \\
\hline $\mathrm{C} 7 \mathrm{~B}-\mathrm{C} 6 \mathrm{~B}-\mathrm{Fe} 1 \mathrm{~B}$ & $70.3(4)$ \\
\hline $\mathrm{C} 7 \mathrm{~B}-\mathrm{C} 6 \mathrm{~B}-\mathrm{H} 6 \mathrm{~B}$ & 124.8 \\
\hline $\mathrm{C} 7 \mathrm{~B}-\mathrm{C} 6 \mathrm{~B}-\mathrm{C} 10 \mathrm{~B}$ & $110.4(8)$ \\
\hline $\mathrm{C} 10 \mathrm{~B}-\mathrm{C} 6 \mathrm{~B}-\mathrm{Fe} 1 \mathrm{~B}$ & $70.8(5)$ \\
\hline $\mathrm{C} 10 \mathrm{~B}-\mathrm{C} 6 \mathrm{~B}-\mathrm{H} 6 \mathrm{~B}$ & 124.8 \\
\hline $\mathrm{Fe} 1 \mathrm{~B}-\mathrm{C} 7 \mathrm{~B}-\mathrm{H} 7 \mathrm{~B}$ & 125.9 \\
\hline $\mathrm{C} 6 \mathrm{~B}-\mathrm{C} 7 \mathrm{~B}-\mathrm{Fe} 1 \mathrm{~B}$ & $69.6(5)$ \\
\hline $\mathrm{C} 6 \mathrm{~B}-\mathrm{C} 7 \mathrm{~B}-\mathrm{H} 7 \mathrm{~B}$ & 127.1 \\
\hline $\mathrm{C} 6 \mathrm{~B}-\mathrm{C} 7 \mathrm{~B}-\mathrm{C} 8 \mathrm{~B}$ & $105.7(10)$ \\
\hline $\mathrm{C} 8 \mathrm{~B}-\mathrm{C} 7 \mathrm{~B}-\mathrm{Fe} 1 \mathrm{~B}$ & $68.9(5)$ \\
\hline $\mathrm{C} 8 \mathrm{~B}-\mathrm{C} 7 \mathrm{~B}-\mathrm{H} 7 \mathrm{~B}$ & 127.1 \\
\hline
\end{tabular}




$\begin{array}{ll}\text { Fe1A-C8A-H8A } & 125.9 \\ \text { C7A-C8A-Fe1A } & 70.2(3) \\ \text { C7A-C8A-H8A } & 125.7 \\ \text { C7A-C8A-C9A } & 108.5(5) \\ \text { C9A-C8A-Fe1A } & 69.7(3) \\ \text { C9A-C8A-H8A } & 125.7 \\ \text { Fe1A-C9A-H9A } & 126.3 \\ \text { C8A-C9A-Fe1A } & 69.5(3) \\ \text { C8A-C9A-H9A } & 126.3 \\ \text { C8A-C9A-C10A } & 107.3(5) \\ \text { C10A-C9A-Fe1A } & 69.4(3) \\ \text { C10A-C9A-H9A } & 126.3 \\ \text { Fe1A-C10A-H10A } & 125.9 \\ \text { C6A-C10A-Fe1A } & 70.2(3) \\ \text { C6A-C10A-C9A } & 108.5(5) \\ \text { C6A-C10A-H10A } & 125.8 \\ \text { C9A-C10A-Fe1A } & 69.7(3) \\ \text { C9A-C10A-H10A } & 125.8 \\ \text { C2A-C11A-H11A } & 116.6 \\ \text { C12A-C11A-C2A } & 126.8(7) \\ \text { C12A-C11A-H11A } & 116.6 \\ \text { C11A-C12A-H12A } & 120.0 \\ \text { C11A-C12A-H12B } & 120.0 \\ \text { H12A-C12A-H12B } & 120.0 \\ \text { P1A-C15A-Fe2A } & 130.6(3) \\ \text { C16A-C15A-Fe2A } & 69.2(3) \\ \text { C16A-C15A-P1A } & 128.0(4) \\ \text { C19A-C15A-Fe2A } & 69.7(3) \\ \text { C19A-C15A-P1A } & 124.2(4) \\ \text { C19A-C15A-C16A } & 107.7(5) \\ \text { C15A-C16A-Fe2A } & 69.0(3) \\ \text { C15A-C16A-C25A } & 127.1(5) \\ \text { C17A-C16A-Fe2A } & 69.8(3) \\ \text { C17A-C16A-C15A } & 106.8(5) \\ \text { C17A-C16A-C25A } & 125.9(5) \\ \text { C25A-C16A-Fe2A } & 130.4(4) \\ \text { Fe2A-C17A-H17A } & 126.4 \\ \text { C16A-C17A-Fe2A } & 69.3(3) \\ \text { C16A-C17A-H17A } & 125.0 \\ \text { C18A-C17A-Fe2A } & 70.8(3) \\ \text { C18A-C17A-C16A } & 109.9(5) \\ \text { C18A-C17A-H17A } & 125.0 \\ \text { Fe2A-C18A-H18A } & 127.6 \\ \text { C17A-C18A-Fe2A } & 69.1(3) \\ \text { C17A-C18A-H18A } & 126.0 \\ \text { C17A-C18A-C19A } & 108.1(5) \\ \text { C19A-C18A-Fe2A } & 69.0(3) \\ \text { C19A-C18A-H18A } & 126.0 \\ & \end{array}$

$\begin{array}{ll}\text { Fe1B-C8B-H8B } & 125.6 \\ \text { C7B-C8B-Fe1B } & 69.7(4) \\ \text { C7B-C8B-H8B } & 125.1 \\ \text { C9B-C8B-Fe1B } & 71.1(5) \\ \text { C9B-C8B-C7B } & 109.8(8) \\ \text { C9B-C8B-H8B } & 125.1 \\ \text { Fe1B-C9B-H9B } & 126.5 \\ \text { C8B-C9B-Fe1B } & 69.0(5) \\ \text { C8B-C9B-H9B } & 125.9 \\ \text { C8B-C9B-C10B } & 108.2(10) \\ \text { C10B-C9B-Fe1B } & 70.2(5) \\ \text { C10B-C9B-H9B } & 125.9 \\ \text { Fe1B-C10B-H10B } & 127.1 \\ \text { C6B-C10B-Fe1B } & 68.1(5) \\ \text { C6B-C10B-H10B } & 127.1 \\ \text { C9B-C10B-Fe1B } & 69.2(5) \\ \text { C9B-C10B-C6B } & 105.9(10) \\ \text { C9B-C10B-H10B } & 127.1 \\ \text { C2B-C11B-H11B } & 118.6 \\ \text { C12B-C11B-C2B } & 122.9(7) \\ \text { C12B-C11B-H11B } & 118.6 \\ \text { C11B-C12B-H12C } & 120.0 \\ \text { C11B-C12B-H12D } & 120.0 \\ \text { H12C-C12B-H12D } & 120.0 \\ \text { P1B-C15B-Fe2B } & 133.1(3) \\ \text { C16B-C15B-Fe2B } & 69.7(4) \\ \text { C16B-C15B-P1B } & 127.3(5) \\ \text { C19B-C15B-Fe2B } & 70.0(3) \\ \text { C19B-C15B-P1B } & 124.5(4) \\ \text { C19B-C15B-C16B } & 107.4(5) \\ \text { C15B-C16B-Fe2B } & 68.4(4) \\ \text { C15B-C16B-C25B } & 124.0(6) \\ \text { C17B-C16B-Fe2B } & 69.5(4) \\ \text { C17B-C16B-C15B } & 106.2(6) \\ \text { C17B-C16B-C25B } & 129.4(6) \\ \text { C25B-C16B-Fe2B } & 131.8(7) \\ \text { Fe2B-C17B-H17B } & 126.5 \\ \text { C16B-C17B-Fe2B } & 69.6(4) \\ \text { C16B-C17B-H17B } & 124.9 \\ \text { C18B-C17B-Fe2B } & 70.7(4) \\ \text { C18B-C17B-C16B } & 110.2(5) \\ \text { C18B-C17B-H17B } & 124.9 \\ \text { Fe2B-C18B-H18B } & 127.0 \\ \text { C17B-C18B-Fe2B } & 69.3(4) \\ \text { C17B-C18B-H18B } & 126.2 \\ \text { C17B-C18B-C19B } & 107.7(6) \\ \text { C19B-C18B-Fe2B } & 69.0(3) \\ \text { C19B-C18B-H18B } & 126.2 \\ & \\ & \end{array}$




\begin{tabular}{|c|c|}
\hline $\mathrm{Fe} 2 \mathrm{~A}-\mathrm{C} 19 \mathrm{~A}-\mathrm{H} 19 \mathrm{~A}$ & 126.4 \\
\hline $\mathrm{C} 15 \mathrm{~A}-\mathrm{C} 19 \mathrm{~A}-\mathrm{Fe} 2 \mathrm{~A}$ & $68.7(3)$ \\
\hline $\mathrm{C} 15 \mathrm{~A}-\mathrm{C} 19 \mathrm{~A}-\mathrm{H} 19 \mathrm{~A}$ & 126.2 \\
\hline $\mathrm{C} 18 \mathrm{~A}-\mathrm{C} 19 \mathrm{~A}-\mathrm{Fe} 2 \mathrm{~A}$ & $70.3(3)$ \\
\hline $\mathrm{C} 18 \mathrm{~A}-\mathrm{C} 19 \mathrm{~A}-\mathrm{C} 15 \mathrm{~A}$ & $107.6(5)$ \\
\hline $\mathrm{C} 18 \mathrm{~A}-\mathrm{C} 19 \mathrm{~A}-\mathrm{H} 19 \mathrm{~A}$ & 126.2 \\
\hline $\mathrm{Fe} 2 \mathrm{~A}-\mathrm{C} 20 \mathrm{~A}-\mathrm{H} 20 \mathrm{~A}$ & 127.1 \\
\hline $\mathrm{C} 21 \mathrm{~A}-\mathrm{C} 20 \mathrm{~A}-\mathrm{Fe} 2 \mathrm{~A}$ & $68.4(4)$ \\
\hline $\mathrm{C} 21 \mathrm{~A}-\mathrm{C} 20 \mathrm{~A}-\mathrm{H} 20 \mathrm{~A}$ & 126.8 \\
\hline $\mathrm{C} 21 \mathrm{~A}-\mathrm{C} 20 \mathrm{~A}-\mathrm{C} 24 \mathrm{~A}$ & $106.3(8)$ \\
\hline $\mathrm{C} 24 \mathrm{~A}-\mathrm{C} 20 \mathrm{~A}-\mathrm{Fe} 2 \mathrm{~A}$ & $69.2(5)$ \\
\hline $\mathrm{C} 24 \mathrm{~A}-\mathrm{C} 20 \mathrm{~A}-\mathrm{H} 20 \mathrm{~A}$ & 126.8 \\
\hline $\mathrm{Fe} 2 \mathrm{~A}-\mathrm{C} 21 \mathrm{~A}-\mathrm{H} 21 \mathrm{~A}$ & 125.5 \\
\hline $\mathrm{C} 20 \mathrm{~A}-\mathrm{C} 21 \mathrm{~A}-\mathrm{Fe} 2 \mathrm{~A}$ & $70.9(4)$ \\
\hline $\mathrm{C} 20 \mathrm{~A}-\mathrm{C} 21 \mathrm{~A}-\mathrm{H} 21 \mathrm{~A}$ & 125.2 \\
\hline $\mathrm{C} 22 \mathrm{~A}-\mathrm{C} 21 \mathrm{~A}-\mathrm{Fe} 2 \mathrm{~A}$ & $69.9(5)$ \\
\hline $\mathrm{C} 22 \mathrm{~A}-\mathrm{C} 21 \mathrm{~A}-\mathrm{C} 20 \mathrm{~A}$ & $109.5(8)$ \\
\hline $\mathrm{C} 22 \mathrm{~A}-\mathrm{C} 21 \mathrm{~A}-\mathrm{H} 21 \mathrm{~A}$ & 125.2 \\
\hline $\mathrm{Fe} 2 \mathrm{~A}-\mathrm{C} 22 \mathrm{~A}-\mathrm{H} 22 \mathrm{~A}$ & 125.5 \\
\hline $\mathrm{C} 21 \mathrm{~A}-\mathrm{C} 22 \mathrm{~A}-\mathrm{Fe} 2 \mathrm{~A}$ & $70.3(4)$ \\
\hline $\mathrm{C} 21 \mathrm{~A}-\mathrm{C} 22 \mathrm{~A}-\mathrm{H} 22 \mathrm{~A}$ & 126.2 \\
\hline $\mathrm{C} 21 \mathrm{~A}-\mathrm{C} 22 \mathrm{~A}-\mathrm{C} 23 \mathrm{~A}$ & $107.5(7)$ \\
\hline $\mathrm{C} 23 \mathrm{~A}-\mathrm{C} 22 \mathrm{~A}-\mathrm{Fe} 2 \mathrm{~A}$ & $69.6(4)$ \\
\hline $\mathrm{C} 23 \mathrm{~A}-\mathrm{C} 22 \mathrm{~A}-\mathrm{H} 22 \mathrm{~A}$ & 126.2 \\
\hline $\mathrm{Fe} 2 \mathrm{~A}-\mathrm{C} 23 \mathrm{~A}-\mathrm{H} 23 \mathrm{~A}$ & 125.5 \\
\hline $\mathrm{C} 22 \mathrm{~A}-\mathrm{C} 23 \mathrm{~A}-\mathrm{Fe} 2 \mathrm{~A}$ & $69.7(4)$ \\
\hline $\mathrm{C} 22 \mathrm{~A}-\mathrm{C} 23 \mathrm{~A}-\mathrm{H} 23 \mathrm{~A}$ & 125.6 \\
\hline $\mathrm{C} 24 \mathrm{~A}-\mathrm{C} 23 \mathrm{~A}-\mathrm{Fe} 2 \mathrm{~A}$ & $70.8(4)$ \\
\hline $\mathrm{C} 24 \mathrm{~A}-\mathrm{C} 23 \mathrm{~A}-\mathrm{C} 22 \mathrm{~A}$ & $108.8(8)$ \\
\hline $\mathrm{C} 24 \mathrm{~A}-\mathrm{C} 23 \mathrm{~A}-\mathrm{H} 23 \mathrm{~A}$ & 125.6 \\
\hline $\mathrm{Fe} 2 \mathrm{~A}-\mathrm{C} 24 \mathrm{~A}-\mathrm{H} 24 \mathrm{~A}$ & 126.2 \\
\hline $\mathrm{C} 20 \mathrm{~A}-\mathrm{C} 24 \mathrm{~A}-\mathrm{Fe} 2 \mathrm{~A}$ & $70.4(4)$ \\
\hline $\mathrm{C} 20 \mathrm{~A}-\mathrm{C} 24 \mathrm{~A}-\mathrm{H} 24 \mathrm{~A}$ & 126.1 \\
\hline $\mathrm{C} 23 \mathrm{~A}-\mathrm{C} 24 \mathrm{~A}-\mathrm{Fe} 2 \mathrm{~A}$ & $69.0(4)$ \\
\hline $\mathrm{C} 23 \mathrm{~A}-\mathrm{C} 24 \mathrm{~A}-\mathrm{C} 20 \mathrm{~A}$ & $107.8(8)$ \\
\hline $\mathrm{C} 23 \mathrm{~A}-\mathrm{C} 24 \mathrm{~A}-\mathrm{H} 24 \mathrm{~A}$ & 126.1 \\
\hline $\mathrm{O} 1 \mathrm{~A}-\mathrm{C} 25 \mathrm{~A}-\mathrm{C} 16 \mathrm{~A}$ & $110.1(5)$ \\
\hline $\mathrm{O} 1 \mathrm{~A}-\mathrm{C} 25 \mathrm{~A}-\mathrm{H} 25 \mathrm{~A}$ & 107.7 \\
\hline $\mathrm{O} 1 \mathrm{~A}-\mathrm{C} 25 \mathrm{~A}-\mathrm{C} 26 \mathrm{~A}$ & $106.4(6)$ \\
\hline $\mathrm{C} 16 \mathrm{~A}-\mathrm{C} 25 \mathrm{~A}-\mathrm{H} 25 \mathrm{~A}$ & 107.7 \\
\hline $\mathrm{C} 26 \mathrm{~A}-\mathrm{C} 25 \mathrm{~A}-\mathrm{C} 16 \mathrm{~A}$ & $116.8(6)$ \\
\hline $\mathrm{C} 26 \mathrm{~A}-\mathrm{C} 25 \mathrm{~A}-\mathrm{H} 25 \mathrm{~A}$ & 107.7 \\
\hline $\mathrm{C} 25 \mathrm{~A}-\mathrm{C} 26 \mathrm{~A}-\mathrm{H} 26 \mathrm{~A}$ & 109.5 \\
\hline $\mathrm{C} 25 \mathrm{~A}-\mathrm{C} 26 \mathrm{~A}-\mathrm{H} 26 \mathrm{~B}$ & 109.5 \\
\hline $\mathrm{C} 25 \mathrm{~A}-\mathrm{C} 26 \mathrm{~A}-\mathrm{H} 26 \mathrm{C}$ & 109.5 \\
\hline $\mathrm{H} 26 \mathrm{~A}-\mathrm{C} 26 \mathrm{~A}-\mathrm{H} 26 \mathrm{~B}$ & 109.5 \\
\hline $\mathrm{H} 26 \mathrm{~A}-\mathrm{C} 26 \mathrm{~A}-\mathrm{H} 26 \mathrm{C}$ & 109.5 \\
\hline & \\
\hline
\end{tabular}

\begin{tabular}{|c|c|}
\hline $\mathrm{Fe} 2 \mathrm{~B}-\mathrm{C} 19 \mathrm{~B}-\mathrm{H} 19 \mathrm{~B}$ & 126.8 \\
\hline $\mathrm{C} 15 \mathrm{~B}-\mathrm{C} 19 \mathrm{~B}-\mathrm{Fe} 2 \mathrm{~B}$ & $68.7(3)$ \\
\hline $\mathrm{C} 15 \mathrm{~B}-\mathrm{C} 19 \mathrm{~B}-\mathrm{H} 19 \mathrm{~B}$ & 125.8 \\
\hline $\mathrm{C} 18 \mathrm{~B}-\mathrm{C} 19 \mathrm{~B}-\mathrm{Fe} 2 \mathrm{~B}$ & $70.3(3)$ \\
\hline $\mathrm{C} 18 \mathrm{~B}-\mathrm{C} 19 \mathrm{~B}-\mathrm{C} 15 \mathrm{~B}$ & $108.4(5)$ \\
\hline $\mathrm{C} 18 \mathrm{~B}-\mathrm{C} 19 \mathrm{~B}-\mathrm{H} 19 \mathrm{~B}$ & 125.8 \\
\hline $\mathrm{Fe} 2 \mathrm{~B}-\mathrm{C} 20 \mathrm{~B}-\mathrm{H} 20 \mathrm{~B}$ & 125.5 \\
\hline $\mathrm{C} 21 \mathrm{~B}-\mathrm{C} 20 \mathrm{~B}-\mathrm{Fe} 2 \mathrm{~B}$ & $69.3(4)$ \\
\hline $\mathrm{C} 21 \mathrm{~B}-\mathrm{C} 20 \mathrm{~B}-\mathrm{H} 20 \mathrm{~B}$ & 126.6 \\
\hline $\mathrm{C} 24 \mathrm{~B}-\mathrm{C} 20 \mathrm{~B}-\mathrm{Fe} 2 \mathrm{~B}$ & $70.1(4)$ \\
\hline $\mathrm{C} 24 \mathrm{~B}-\mathrm{C} 20 \mathrm{~B}-\mathrm{H} 20 \mathrm{~B}$ & 126.6 \\
\hline $\mathrm{C} 24 \mathrm{~B}-\mathrm{C} 20 \mathrm{~B}-\mathrm{C} 21 \mathrm{~B}$ & $106.7(8)$ \\
\hline $\mathrm{Fe} 2 \mathrm{~B}-\mathrm{C} 21 \mathrm{~B}-\mathrm{H} 21 \mathrm{~B}$ & 126.1 \\
\hline $\mathrm{C} 20 \mathrm{~B}-\mathrm{C} 21 \mathrm{~B}-\mathrm{Fe} 2 \mathrm{~B}$ & $69.2(4)$ \\
\hline $\mathrm{C} 20 \mathrm{~B}-\mathrm{C} 21 \mathrm{~B}-\mathrm{H} 21 \mathrm{~B}$ & 126.8 \\
\hline $\mathrm{C} 22 \mathrm{~B}-\mathrm{C} 21 \mathrm{~B}-\mathrm{Fe} 2 \mathrm{~B}$ & $69.4(5)$ \\
\hline $\mathrm{C} 22 \mathrm{~B}-\mathrm{C} 21 \mathrm{~B}-\mathrm{C} 20 \mathrm{~B}$ & $106.3(10)$ \\
\hline $\mathrm{C} 22 \mathrm{~B}-\mathrm{C} 21 \mathrm{~B}-\mathrm{H} 21 \mathrm{~B}$ & 126.8 \\
\hline $\mathrm{Fe} 2 \mathrm{~B}-\mathrm{C} 22 \mathrm{~B}-\mathrm{H} 22 \mathrm{~B}$ & 125.4 \\
\hline $\mathrm{C} 21 \mathrm{~B}-\mathrm{C} 22 \mathrm{~B}-\mathrm{Fe} 2 \mathrm{~B}$ & $70.5(6)$ \\
\hline $\mathrm{C} 21 \mathrm{~B}-\mathrm{C} 22 \mathrm{~B}-\mathrm{H} 22 \mathrm{~B}$ & 125.4 \\
\hline $\mathrm{C} 23 \mathrm{~B}-\mathrm{C} 22 \mathrm{~B}-\mathrm{Fe} 2 \mathrm{~B}$ & $70.4(5)$ \\
\hline $\mathrm{C} 23 \mathrm{~B}-\mathrm{C} 22 \mathrm{~B}-\mathrm{C} 21 \mathrm{~B}$ & $109.3(9)$ \\
\hline $\mathrm{C} 23 \mathrm{~B}-\mathrm{C} 22 \mathrm{~B}-\mathrm{H} 22 \mathrm{~B}$ & 125.4 \\
\hline $\mathrm{Fe} 2 \mathrm{~B}-\mathrm{C} 23 \mathrm{~B}-\mathrm{H} 23 \mathrm{~B}$ & 125.1 \\
\hline $\mathrm{C} 22 \mathrm{~B}-\mathrm{C} 23 \mathrm{~B}-\mathrm{Fe} 2 \mathrm{~B}$ & $70.3(7)$ \\
\hline $\mathrm{C} 22 \mathrm{~B}-\mathrm{C} 23 \mathrm{~B}-\mathrm{H} 23 \mathrm{~B}$ & 125.7 \\
\hline $\mathrm{C} 22 \mathrm{~B}-\mathrm{C} 23 \mathrm{~B}-\mathrm{C} 24 \mathrm{~B}$ & $108.7(11)$ \\
\hline $\mathrm{C} 24 \mathrm{~B}-\mathrm{C} 23 \mathrm{~B}-\mathrm{Fe} 2 \mathrm{~B}$ & $70.5(5)$ \\
\hline $\mathrm{C} 24 \mathrm{~B}-\mathrm{C} 23 \mathrm{~B}-\mathrm{H} 23 \mathrm{~B}$ & 125.7 \\
\hline $\mathrm{Fe} 2 \mathrm{~B}-\mathrm{C} 24 \mathrm{~B}-\mathrm{H} 24 \mathrm{~B}$ & 126.2 \\
\hline $\mathrm{C} 20 \mathrm{~B}-\mathrm{C} 24 \mathrm{~B}-\mathrm{Fe} 2 \mathrm{~B}$ & $70.2(5)$ \\
\hline $\mathrm{C} 20 \mathrm{~B}-\mathrm{C} 24 \mathrm{~B}-\mathrm{C} 23 \mathrm{~B}$ & $109.0(10)$ \\
\hline $\mathrm{C} 20 \mathrm{~B}-\mathrm{C} 24 \mathrm{~B}-\mathrm{H} 24 \mathrm{~B}$ & 125.5 \\
\hline $\mathrm{C} 23 \mathrm{~B}-\mathrm{C} 24 \mathrm{~B}-\mathrm{Fe} 2 \mathrm{~B}$ & $69.6(6)$ \\
\hline $\mathrm{C} 23 \mathrm{~B}-\mathrm{C} 24 \mathrm{~B}-\mathrm{H} 24 \mathrm{~B}$ & 125.5 \\
\hline $\mathrm{O} 1 \mathrm{~B}-\mathrm{C} 25 \mathrm{~B}-\mathrm{C} 16 \mathrm{~B}$ & $109.3(8)$ \\
\hline $\mathrm{O} 1 \mathrm{~B}-\mathrm{C} 25 \mathrm{~B}-\mathrm{H} 25 \mathrm{~B}$ & 110.3 \\
\hline $\mathrm{O} 1 \mathrm{~B}-\mathrm{C} 25 \mathrm{~B}-\mathrm{C} 26 \mathrm{~B}$ & $105.5(7)$ \\
\hline $\mathrm{C} 16 \mathrm{~B}-\mathrm{C} 25 \mathrm{~B}-\mathrm{H} 25 \mathrm{~B}$ & 110.3 \\
\hline $\mathrm{C} 16 \mathrm{~B}-\mathrm{C} 25 \mathrm{~B}-\mathrm{C} 26 \mathrm{~B}$ & $111.0(9)$ \\
\hline $\mathrm{C} 26 \mathrm{~B}-\mathrm{C} 25 \mathrm{~B}-\mathrm{H} 25 \mathrm{~B}$ & 110.3 \\
\hline $\mathrm{C} 25 \mathrm{~B}-\mathrm{C} 26 \mathrm{~B}-\mathrm{H} 26 \mathrm{D}$ & 109.5 \\
\hline $\mathrm{C} 25 \mathrm{~B}-\mathrm{C} 26 \mathrm{~B}-\mathrm{H} 26 \mathrm{E}$ & 109.5 \\
\hline $\mathrm{C} 25 \mathrm{~B}-\mathrm{C} 26 \mathrm{~B}-\mathrm{H} 26 \mathrm{~F}$ & 109.5 \\
\hline $\mathrm{H} 26 \mathrm{D}-\mathrm{C} 26 \mathrm{~B}-\mathrm{H} 26 \mathrm{E}$ & 109.5 \\
\hline $\mathrm{H} 26 \mathrm{D}-\mathrm{C} 26 \mathrm{~B}-\mathrm{H} 26 \mathrm{~F}$ & 109.5 \\
\hline $\mathrm{H} 26 \mathrm{E}-\mathrm{C} 26 \mathrm{~B}-\mathrm{H} 26 \mathrm{~F}$ & 109.5 \\
\hline
\end{tabular}




\begin{tabular}{|c|c|}
\hline $\mathrm{C} 35 \mathrm{~A}-\mathrm{C} 34 \mathrm{~A}-\mathrm{P} 1 \mathrm{~A}$ & $121.3(4)$ \\
\hline $\mathrm{C} 39 \mathrm{~A}-\mathrm{C} 34 \mathrm{~A}-\mathrm{P} 1 \mathrm{~A}$ & $119.8(4)$ \\
\hline $\mathrm{C} 39 \mathrm{~A}-\mathrm{C} 34 \mathrm{~A}-\mathrm{C} 35 \mathrm{~A}$ & $119.0(5)$ \\
\hline $\mathrm{C} 34 \mathrm{~A}-\mathrm{C} 35 \mathrm{~A}-\mathrm{H} 35 \mathrm{~A}$ & 119.8 \\
\hline $\mathrm{C} 36 \mathrm{~A}-\mathrm{C} 35 \mathrm{~A}-\mathrm{C} 34 \mathrm{~A}$ & $120.5(6)$ \\
\hline $\mathrm{C} 36 \mathrm{~A}-\mathrm{C} 35 \mathrm{~A}-\mathrm{H} 35 \mathrm{~A}$ & 119.8 \\
\hline $\mathrm{C} 35 \mathrm{~A}-\mathrm{C} 36 \mathrm{~A}-\mathrm{H} 36 \mathrm{~A}$ & 120.3 \\
\hline $\mathrm{C} 37 \mathrm{~A}-\mathrm{C} 36 \mathrm{~A}-\mathrm{C} 35 \mathrm{~A}$ & $119.5(6)$ \\
\hline $\mathrm{C} 37 \mathrm{~A}-\mathrm{C} 36 \mathrm{~A}-\mathrm{H} 36 \mathrm{~A}$ & 120.3 \\
\hline $\mathrm{C} 36 \mathrm{~A}-\mathrm{C} 37 \mathrm{~A}-\mathrm{H} 37 \mathrm{~A}$ & 120.0 \\
\hline $\mathrm{C} 38 \mathrm{~A}-\mathrm{C} 37 \mathrm{~A}-\mathrm{C} 36 \mathrm{~A}$ & $120.1(6)$ \\
\hline $\mathrm{C} 38 \mathrm{~A}-\mathrm{C} 37 \mathrm{~A}-\mathrm{H} 37 \mathrm{~A}$ & 120.0 \\
\hline $\mathrm{C} 37 \mathrm{~A}-\mathrm{C} 38 \mathrm{~A}-\mathrm{H} 38 \mathrm{~A}$ & 119.4 \\
\hline $\mathrm{C} 37 \mathrm{~A}-\mathrm{C} 38 \mathrm{~A}-\mathrm{C} 39 \mathrm{~A}$ & $121.2(6)$ \\
\hline $\mathrm{C} 39 \mathrm{~A}-\mathrm{C} 38 \mathrm{~A}-\mathrm{H} 38 \mathrm{~A}$ & 119.4 \\
\hline $\mathrm{C} 34 \mathrm{~A}-\mathrm{C} 39 \mathrm{~A}-\mathrm{H} 39 \mathrm{~A}$ & 120.1 \\
\hline $\mathrm{C} 38 \mathrm{~A}-\mathrm{C} 39 \mathrm{~A}-\mathrm{C} 34 \mathrm{~A}$ & $119.9(6)$ \\
\hline $\mathrm{C} 38 \mathrm{~A}-\mathrm{C} 39 \mathrm{~A}-\mathrm{H} 39 \mathrm{~A}$ & 120.1 \\
\hline $\mathrm{Fe} 1 \mathrm{~A}-\mathrm{C} 1 \mathrm{~A}-\mathrm{C} 2 \mathrm{~A}-\mathrm{C} 3 \mathrm{~A}$ & $59.0(4)$ \\
\hline $\mathrm{Fe} 1 \mathrm{~A}-\mathrm{C} 1 \mathrm{~A}-\mathrm{C} 2 \mathrm{~A}-\mathrm{C} 11 \mathrm{~A}$ & $-115.1(6)$ \\
\hline $\mathrm{Fe} 1 \mathrm{~A}-\mathrm{C} 1 \mathrm{~A}-\mathrm{C} 5 \mathrm{~A}-\mathrm{C} 4 \mathrm{~A}$ & $-59.6(4)$ \\
\hline $\mathrm{Fe} 1 \mathrm{~A}-\mathrm{C} 2 \mathrm{~A}-\mathrm{C} 3 \mathrm{~A}-\mathrm{C} 4 \mathrm{~A}$ & $59.4(4)$ \\
\hline $\mathrm{Fe} 1 \mathrm{~A}-\mathrm{C} 2 \mathrm{~A}-\mathrm{C} 11 \mathrm{~A}-\mathrm{C} 12 \mathrm{~A}$ & $86.6(9)$ \\
\hline $\mathrm{Fe} 1 \mathrm{~A}-\mathrm{C} 3 \mathrm{~A}-\mathrm{C} 4 \mathrm{~A}-\mathrm{C} 5 \mathrm{~A}$ & $58.0(4)$ \\
\hline $\mathrm{Fe} 1 \mathrm{~A}-\mathrm{C} 4 \mathrm{~A}-\mathrm{C} 5 \mathrm{~A}-\mathrm{C} 1 \mathrm{~A}$ & $58.6(4)$ \\
\hline $\mathrm{Fe} 1 \mathrm{~A}-\mathrm{C} 6 \mathrm{~A}-\mathrm{C} 7 \mathrm{~A}-\mathrm{C} 8 \mathrm{~A}$ & $-58.9(4)$ \\
\hline $\mathrm{Fe} 1 \mathrm{~A}-\mathrm{C} 6 \mathrm{~A}-\mathrm{C} 10 \mathrm{~A}-\mathrm{C} 9 \mathrm{~A}$ & $59.4(4)$ \\
\hline $\mathrm{Fe} 1 \mathrm{~A}-\mathrm{C} 7 \mathrm{~A}-\mathrm{C} 8 \mathrm{~A}-\mathrm{C} 9 \mathrm{~A}$ & $-59.4(4)$ \\
\hline $\mathrm{Fe} 1 \mathrm{~A}-\mathrm{C} 8 \mathrm{~A}-\mathrm{C} 9 \mathrm{~A}-\mathrm{C} 10 \mathrm{~A}$ & $-59.3(4)$ \\
\hline $\mathrm{Fe} 1 \mathrm{~A}-\mathrm{C} 9 \mathrm{~A}-\mathrm{C} 10 \mathrm{~A}-\mathrm{C} 6 \mathrm{~A}$ & $-59.7(4)$ \\
\hline $\mathrm{Fe} 2 \mathrm{~A}-\mathrm{C} 15 \mathrm{~A}-\mathrm{C} 16 \mathrm{~A}-\mathrm{C} 17 \mathrm{~A}$ & $59.8(4)$ \\
\hline $\mathrm{Fe} 2 \mathrm{~A}-\mathrm{C} 15 \mathrm{~A}-\mathrm{C} 16 \mathrm{~A}-\mathrm{C} 25 \mathrm{~A}$ & $-125.4(5)$ \\
\hline $\mathrm{Fe} 2 \mathrm{~A}-\mathrm{C} 15 \mathrm{~A}-\mathrm{C} 19 \mathrm{~A}-\mathrm{C} 18 \mathrm{~A}$ & $-59.8(4)$ \\
\hline $\mathrm{Fe} 2 \mathrm{~A}-\mathrm{C} 16 \mathrm{~A}-\mathrm{C} 17 \mathrm{~A}-\mathrm{C} 18 \mathrm{~A}$ & $59.3(4)$ \\
\hline $\mathrm{Fe} 2 \mathrm{~A}-\mathrm{C} 16 \mathrm{~A}-\mathrm{C} 25 \mathrm{~A}-\mathrm{O} 1 \mathrm{~A}$ & $-174.7(4)$ \\
\hline $\mathrm{Fe} 2 \mathrm{~A}-\mathrm{C} 16 \mathrm{~A}-\mathrm{C} 25 \mathrm{~A}-\mathrm{C} 26 \mathrm{~A}$ & $63.9(8)$ \\
\hline $\mathrm{Fe} 2 \mathrm{~A}-\mathrm{C} 17 \mathrm{~A}-\mathrm{C} 18 \mathrm{~A}-\mathrm{C} 19 \mathrm{~A}$ & $58.1(4)$ \\
\hline $\mathrm{Fe} 2 \mathrm{~A}-\mathrm{C} 18 \mathrm{~A}-\mathrm{C} 19 \mathrm{~A}-\mathrm{C} 15 \mathrm{~A}$ & $58.8(4)$ \\
\hline $\mathrm{Fe} 2 \mathrm{~A}-\mathrm{C} 20 \mathrm{~A}-\mathrm{C} 21 \mathrm{~A}-\mathrm{C} 22 \mathrm{~A}$ & $-59.4(5)$ \\
\hline $\mathrm{Fe} 2 \mathrm{~A}-\mathrm{C} 20 \mathrm{~A}-\mathrm{C} 24 \mathrm{~A}-\mathrm{C} 23 \mathrm{~A}$ & $59.0(5)$ \\
\hline $\mathrm{Fe} 2 \mathrm{~A}-\mathrm{C} 21 \mathrm{~A}-\mathrm{C} 22 \mathrm{~A}-\mathrm{C} 23 \mathrm{~A}$ & $-59.9(5)$ \\
\hline $\mathrm{Fe} 2 \mathrm{~A}-\mathrm{C} 22 \mathrm{~A}-\mathrm{C} 23 \mathrm{~A}-\mathrm{C} 24 \mathrm{~A}$ & $-60.2(5)$ \\
\hline $\mathrm{Fe} 2 \mathrm{~A}-\mathrm{C} 23 \mathrm{~A}-\mathrm{C} 24 \mathrm{~A}-\mathrm{C} 20 \mathrm{~A}$ & $-59.9(5)$ \\
\hline $\mathrm{S} 1 \mathrm{~A}-\mathrm{P} 1 \mathrm{~A}-\mathrm{C} 1 \mathrm{~A}-\mathrm{Fe} 1 \mathrm{~A}$ & $58.3(4)$ \\
\hline $\mathrm{S} 1 \mathrm{~A}-\mathrm{P} 1 \mathrm{~A}-\mathrm{C} 1 \mathrm{~A}-\mathrm{C} 2 \mathrm{~A}$ & $-35.4(6)$ \\
\hline $\mathrm{S} 1 \mathrm{~A}-\mathrm{P} 1 \mathrm{~A}-\mathrm{C} 1 \mathrm{~A}-\mathrm{C} 5 \mathrm{~A}$ & $148.4(4)$ \\
\hline $\mathrm{S} 1 \mathrm{~A}-\mathrm{P} 1 \mathrm{~A}-\mathrm{C} 15 \mathrm{~A}-\mathrm{Fe} 2 \mathrm{~A}$ & $45.8(4)$ \\
\hline
\end{tabular}

$\begin{array}{ll}\mathrm{C} 35 \mathrm{~B}-\mathrm{C} 34 \mathrm{~B}-\mathrm{P} 1 \mathrm{~B} & 120.6(5) \\ \mathrm{C} 39 \mathrm{~B}-\mathrm{C} 34 \mathrm{~B}-\mathrm{P} 1 \mathrm{~B} & 119.8(4) \\ \mathrm{C} 39 \mathrm{~B}-\mathrm{C} 34 \mathrm{~B}-\mathrm{C} 35 \mathrm{~B} & 119.6(6) \\ \mathrm{C} 34 \mathrm{~B}-\mathrm{C} 35 \mathrm{~B}-\mathrm{H} 35 \mathrm{~B} & 119.9 \\ \mathrm{C} 36 \mathrm{~B}-\mathrm{C} 35 \mathrm{~B}-\mathrm{C} 34 \mathrm{~B} & 120.3(6) \\ \mathrm{C} 36 \mathrm{~B}-\mathrm{C} 35 \mathrm{~B}-\mathrm{H} 35 \mathrm{~B} & 119.9 \\ \mathrm{C} 35 \mathrm{~B}-\mathrm{C} 36 \mathrm{~B}-\mathrm{H} 36 \mathrm{~B} & 120.2 \\ \mathrm{C} 37 \mathrm{~B}-\mathrm{C} 36 \mathrm{~B}-\mathrm{C} 35 \mathrm{~B} & 119.6(6) \\ \mathrm{C} 37 \mathrm{~B}-\mathrm{C} 36 \mathrm{~B}-\mathrm{H} 36 \mathrm{~B} & 120.2 \\ \mathrm{C} 36 \mathrm{~B}-\mathrm{C} 37 \mathrm{~B}-\mathrm{H} 37 \mathrm{~B} & 119.7 \\ \mathrm{C} 36 \mathrm{~B}-\mathrm{C} 37 \mathrm{~B}-\mathrm{C} 38 \mathrm{~B} & 120.6(6) \\ \mathrm{C} 38 \mathrm{~B}-\mathrm{C} 37 \mathrm{~B}-\mathrm{H} 37 \mathrm{~B} & 119.7 \\ \mathrm{C} 37 \mathrm{~B}-\mathrm{C} 38 \mathrm{~B}-\mathrm{H} 38 \mathrm{~B} & 120.3 \\ \text { C37B-C38B-C39B} & 119.5(6) \\ \text { C39B-C38B-H38B } & 120.3 \\ \text { C34B-C39B-C38B } & 120.4(6) \\ \text { C34B-C39B-H39B } & 119.8 \\ \text { C38B-C39B-H39B } & 119.8\end{array}$

$\mathrm{Fe} 1 \mathrm{~B}-\mathrm{C} 1 \mathrm{~B}-\mathrm{C} 2 \mathrm{~B}-\mathrm{C} 3 \mathrm{~B} \quad 59.0(4)$

$\mathrm{Fe} 1 \mathrm{~B}-\mathrm{C} 1 \mathrm{~B}-\mathrm{C} 2 \mathrm{~B}-\mathrm{C} 11 \mathrm{~B} \quad-121.1$ (7)

$\mathrm{Fe} 1 \mathrm{~B}-\mathrm{C} 1 \mathrm{~B}-\mathrm{C} 5 \mathrm{~B}-\mathrm{C} 4 \mathrm{~B} \quad-59.4$ (4)

$\mathrm{Fe} 1 \mathrm{~B}-\mathrm{C} 2 \mathrm{~B}-\mathrm{C} 3 \mathrm{~B}-\mathrm{C} 4 \mathrm{~B} \quad 57.7$ (4)

$\mathrm{Fe} 1 \mathrm{~B}-\mathrm{C} 2 \mathrm{~B}-\mathrm{C} 11 \mathrm{~B}-\mathrm{C} 12 \mathrm{~B} \quad 115.3(8)$

$\mathrm{Fe} 1 \mathrm{~B}-\mathrm{C} 3 \mathrm{~B}-\mathrm{C} 4 \mathrm{~B}-\mathrm{C} 5 \mathrm{~B} \quad 59.1$ (4)

$\mathrm{Fe} 1 \mathrm{~B}-\mathrm{C} 4 \mathrm{~B}-\mathrm{C} 5 \mathrm{~B}-\mathrm{C} 1 \mathrm{~B} \quad 58.9(4)$

$\mathrm{Fe} 1 \mathrm{~B}-\mathrm{C} 6 \mathrm{~B}-\mathrm{C} 7 \mathrm{~B}-\mathrm{C} 8 \mathrm{~B} \quad-59.7(5)$

$\mathrm{Fe} 1 \mathrm{~B}-\mathrm{C} 6 \mathrm{~B}-\mathrm{C} 10 \mathrm{~B}-\mathrm{C} 9 \mathrm{~B} \quad 59.2(6)$

$\mathrm{Fe} 1 \mathrm{~B}-\mathrm{C} 7 \mathrm{~B}-\mathrm{C} 8 \mathrm{~B}-\mathrm{C} 9 \mathrm{~B} \quad-60.0(6)$

$\mathrm{Fe} 1 \mathrm{~B}-\mathrm{C} 8 \mathrm{~B}-\mathrm{C} 9 \mathrm{~B}-\mathrm{C} 10 \mathrm{~B} \quad-59.5(6)$

$\mathrm{Fe} 1 \mathrm{~B}-\mathrm{C} 9 \mathrm{~B}-\mathrm{C} 10 \mathrm{~B}-\mathrm{C} 6 \mathrm{~B} \quad-58.5(5)$

$\mathrm{Fe} 2 \mathrm{~B}-\mathrm{C} 15 \mathrm{~B}-\mathrm{C} 16 \mathrm{~B}-\mathrm{C} 17 \mathrm{~B} \quad 59.5(5)$

$\mathrm{Fe} 2 \mathrm{~B}-\mathrm{C} 15 \mathrm{~B}-\mathrm{C} 16 \mathrm{~B}-\mathrm{C} 25 \mathrm{~B}-126.8$ (9)

$\mathrm{Fe} 2 \mathrm{~B}-\mathrm{C} 15 \mathrm{~B}-\mathrm{C} 19 \mathrm{~B}-\mathrm{C} 18 \mathrm{~B} \quad-59.2(4)$

$\mathrm{Fe} 2 \mathrm{~B}-\mathrm{C} 16 \mathrm{~B}-\mathrm{C} 17 \mathrm{~B}-\mathrm{C} 18 \mathrm{~B} \quad 59.1$ (5)

$\mathrm{Fe} 2 \mathrm{~B}-\mathrm{C} 16 \mathrm{~B}-\mathrm{C} 25 \mathrm{~B}-\mathrm{O} 1 \mathrm{~B} \quad-175.1$ (5)

$\mathrm{Fe} 2 \mathrm{~B}-\mathrm{C} 16 \mathrm{~B}-\mathrm{C} 25 \mathrm{~B}-\mathrm{C} 26 \mathrm{~B} \quad 68.9(9)$

$\mathrm{Fe} 2 \mathrm{~B}-\mathrm{C} 17 \mathrm{~B}-\mathrm{C} 18 \mathrm{~B}-\mathrm{C} 19 \mathrm{~B} \quad 58.6(4)$

$\mathrm{Fe} 2 \mathrm{~B}-\mathrm{C} 18 \mathrm{~B}-\mathrm{C} 19 \mathrm{~B}-\mathrm{C} 15 \mathrm{~B} \quad 58.2(4)$

$\mathrm{Fe} 2 \mathrm{~B}-\mathrm{C} 20 \mathrm{~B}-\mathrm{C} 21 \mathrm{~B}-\mathrm{C} 22 \mathrm{~B} \quad-59.8(6)$

$\mathrm{Fe} 2 \mathrm{~B}-\mathrm{C} 20 \mathrm{~B}-\mathrm{C} 24 \mathrm{~B}-\mathrm{C} 23 \mathrm{~B} \quad 59.0(6)$

$\mathrm{Fe} 2 \mathrm{~B}-\mathrm{C} 21 \mathrm{~B}-\mathrm{C} 22 \mathrm{~B}-\mathrm{C} 23 \mathrm{~B}-59.9(6)$

$\mathrm{Fe} 2 \mathrm{~B}-\mathrm{C} 22 \mathrm{~B}-\mathrm{C} 23 \mathrm{~B}-\mathrm{C} 24 \mathrm{~B}-60.3(6)$

$\mathrm{Fe} 2 \mathrm{~B}-\mathrm{C} 23 \mathrm{~B}-\mathrm{C} 24 \mathrm{~B}-\mathrm{C} 20 \mathrm{~B} \quad-59.4(6)$

$\mathrm{S} 1 \mathrm{~B}-\mathrm{P} 1 \mathrm{~B}-\mathrm{C} 1 \mathrm{~B}-\mathrm{Fe} 1 \mathrm{~B} \quad 57.4$ (4)

$\mathrm{S} 1 \mathrm{~B}-\mathrm{P} 1 \mathrm{~B}-\mathrm{C} 1 \mathrm{~B}-\mathrm{C} 2 \mathrm{~B} \quad-36.8(6)$

$\mathrm{S} 1 \mathrm{~B}-\mathrm{P} 1 \mathrm{~B}-\mathrm{C} 1 \mathrm{~B}-\mathrm{C} 5 \mathrm{~B} \quad 145.2$ (4)

S1B-P1B-C15B-Fe2B 46.2 (5) 


\begin{tabular}{|c|c|c|c|}
\hline $\mathrm{S} 1 \mathrm{~A}-\mathrm{P} 1 \mathrm{~A}-\mathrm{C} 15 \mathrm{~A}-\mathrm{C} 16 \mathrm{~A}$ & $-48.6(5)$ & $\mathrm{S} 1 \mathrm{~B}-\mathrm{P} 1 \mathrm{~B}-\mathrm{C} 15 \mathrm{~B}-\mathrm{C} 16 \mathrm{~B}$ & $-50.4(6)$ \\
\hline $\mathrm{S} 1 \mathrm{~A}-\mathrm{P} 1 \mathrm{~A}-\mathrm{C} 15 \mathrm{~A}-\mathrm{C} 19 \mathrm{~A}$ & $137.5(4)$ & $\mathrm{S} 1 \mathrm{~B}-\mathrm{P} 1 \mathrm{~B}-\mathrm{C} 15 \mathrm{~B}-\mathrm{C} 19 \mathrm{~B}$ & $140.5(4)$ \\
\hline $\mathrm{S} 1 \mathrm{~A}-\mathrm{P} 1 \mathrm{~A}-\mathrm{C} 34 \mathrm{~A}-\mathrm{C} 35 \mathrm{~A}$ & $157.1(4)$ & $\mathrm{S} 1 \mathrm{~B}-\mathrm{P} 1 \mathrm{~B}-\mathrm{C} 34 \mathrm{~B}-\mathrm{C} 35 \mathrm{~B}$ & $155.8(4)$ \\
\hline $\mathrm{S} 1 \mathrm{~A}-\mathrm{P} 1 \mathrm{~A}-\mathrm{C} 34 \mathrm{~A}-\mathrm{C} 39 \mathrm{~A}$ & $-22.1(4)$ & $\mathrm{S} 1 \mathrm{~B}-\mathrm{P} 1 \mathrm{~B}-\mathrm{C} 34 \mathrm{~B}-\mathrm{C} 39 \mathrm{~B}$ & $-25.5(5)$ \\
\hline $\mathrm{P} 1 \mathrm{~A}-\mathrm{C} 1 \mathrm{~A}-\mathrm{C} 2 \mathrm{~A}-\mathrm{Fe} 1 \mathrm{~A}$ & $123.8(5)$ & $\mathrm{P} 1 \mathrm{~B}-\mathrm{C} 1 \mathrm{~B}-\mathrm{C} 2 \mathrm{~B}-\mathrm{Fe} 1 \mathrm{~B}$ & $122.2(5)$ \\
\hline $\mathrm{P} 1 \mathrm{~A}-\mathrm{C} 1 \mathrm{~A}-\mathrm{C} 2 \mathrm{~A}-\mathrm{C} 3 \mathrm{~A}$ & $-177.2(4)$ & $\mathrm{P} 1 \mathrm{~B}-\mathrm{C} 1 \mathrm{~B}-\mathrm{C} 2 \mathrm{~B}-\mathrm{C} 3 \mathrm{~B}$ & $-178.9(4)$ \\
\hline $\mathrm{P} 1 \mathrm{~A}-\mathrm{C} 1 \mathrm{~A}-\mathrm{C} 2 \mathrm{~A}-\mathrm{C} 11 \mathrm{~A}$ & $8.7(9)$ & $\mathrm{P} 1 \mathrm{~B}-\mathrm{C} 1 \mathrm{~B}-\mathrm{C} 2 \mathrm{~B}-\mathrm{C} 11 \mathrm{~B}$ & $1.1(10)$ \\
\hline $\mathrm{P} 1 \mathrm{~A}-\mathrm{C} 1 \mathrm{~A}-\mathrm{C} 5 \mathrm{~A}-\mathrm{Fe} 1 \mathrm{~A}$ & $-123.5(4)$ & $\mathrm{P} 1 \mathrm{~B}-\mathrm{C} 1 \mathrm{~B}-\mathrm{C} 5 \mathrm{~B}-\mathrm{Fe} 1 \mathrm{~B}$ & $-121.3(4)$ \\
\hline $\mathrm{P} 1 \mathrm{~A}-\mathrm{C} 1 \mathrm{~A}-\mathrm{C} 5 \mathrm{~A}-\mathrm{C} 4 \mathrm{~A}$ & $176.8(4)$ & $\mathrm{P} 1 \mathrm{~B}-\mathrm{C} 1 \mathrm{~B}-\mathrm{C} 5 \mathrm{~B}-\mathrm{C} 4 \mathrm{~B}$ & $179.4(4)$ \\
\hline $\mathrm{P} 1 \mathrm{~A}-\mathrm{C} 15 \mathrm{~A}-\mathrm{C} 16 \mathrm{~A}-\mathrm{Fe} 2 \mathrm{~A}$ & $125.9(4)$ & $\mathrm{P} 1 \mathrm{~B}-\mathrm{C} 15 \mathrm{~B}-\mathrm{C} 16 \mathrm{~B}-\mathrm{Fe} 2 \mathrm{~B}$ & $129.3(5)$ \\
\hline $\mathrm{P} 1 \mathrm{~A}-\mathrm{C} 15 \mathrm{~A}-\mathrm{C} 16 \mathrm{~A}-\mathrm{C} 17 \mathrm{~A}$ & $-174.2(4)$ & $\mathrm{P} 1 \mathrm{~B}-\mathrm{C} 15 \mathrm{~B}-\mathrm{C} 16 \mathrm{~B}-\mathrm{C} 17 \mathrm{~B}$ & $-171.2(5)$ \\
\hline $\mathrm{P} 1 \mathrm{~A}-\mathrm{C} 15 \mathrm{~A}-\mathrm{C} 16 \mathrm{~A}-\mathrm{C} 25 \mathrm{~A}$ & $0.5(8)$ & $\mathrm{P} 1 \mathrm{~B}-\mathrm{C} 15 \mathrm{~B}-\mathrm{C} 16 \mathrm{~B}-\mathrm{C} 25 \mathrm{~B}$ & $2.5(11)$ \\
\hline $\mathrm{P} 1 \mathrm{~A}-\mathrm{C} 15 \mathrm{~A}-\mathrm{C} 19 \mathrm{~A}-\mathrm{Fe} 2 \mathrm{~A}$ & $-126.0(4)$ & $\mathrm{P} 1 \mathrm{~B}-\mathrm{C} 15 \mathrm{~B}-\mathrm{C} 19 \mathrm{~B}-\mathrm{Fe} 2 \mathrm{~B}$ & $-129.2(4)$ \\
\hline $\mathrm{P} 1 \mathrm{~A}-\mathrm{C} 15 \mathrm{~A}-\mathrm{C} 19 \mathrm{~A}-\mathrm{C} 18 \mathrm{~A}$ & $174.3(4)$ & $\mathrm{P} 1 \mathrm{~B}-\mathrm{C} 15 \mathrm{~B}-\mathrm{C} 19 \mathrm{~B}-\mathrm{C} 18 \mathrm{~B}$ & $171.6(4)$ \\
\hline $\mathrm{P} 1 \mathrm{~A}-\mathrm{C} 34 \mathrm{~A}-\mathrm{C} 35 \mathrm{~A}-\mathrm{C} 36 \mathrm{~A}$ & $-179.7(4)$ & $\mathrm{P} 1 \mathrm{~B}-\mathrm{C} 34 \mathrm{~B}-\mathrm{C} 35 \mathrm{~B}-\mathrm{C} 36 \mathrm{~B}$ & $178.6(5)$ \\
\hline $\mathrm{P} 1 \mathrm{~A}-\mathrm{C} 34 \mathrm{~A}-\mathrm{C} 39 \mathrm{~A}-\mathrm{C} 38 \mathrm{~A}$ & $179.4(4)$ & $\mathrm{P} 1 \mathrm{~B}-\mathrm{C} 34 \mathrm{~B}-\mathrm{C} 39 \mathrm{~B}-\mathrm{C} 38 \mathrm{~B}$ & $-177.6(5)$ \\
\hline $\mathrm{C} 1 \mathrm{~A}-\mathrm{P} 1 \mathrm{~A}-\mathrm{C} 15 \mathrm{~A}-\mathrm{Fe} 2 \mathrm{~A}$ & $-83.8(4)$ & $\mathrm{C} 1 \mathrm{~B}-\mathrm{P} 1 \mathrm{~B}-\mathrm{C} 15 \mathrm{~B}-\mathrm{Fe} 2 \mathrm{~B}$ & $-84.5(5)$ \\
\hline $\mathrm{C} 1 \mathrm{~A}-\mathrm{P} 1 \mathrm{~A}-\mathrm{C} 15 \mathrm{~A}-\mathrm{C} 16 \mathrm{~A}$ & $-178.2(4)$ & $\mathrm{C} 1 \mathrm{~B}-\mathrm{P} 1 \mathrm{~B}-\mathrm{C} 15 \mathrm{~B}-\mathrm{C} 16 \mathrm{~B}$ & $178.9(5)$ \\
\hline $\mathrm{C} 1 \mathrm{~A}-\mathrm{P} 1 \mathrm{~A}-\mathrm{C} 15 \mathrm{~A}-\mathrm{C} 19 \mathrm{~A}$ & $7.9(5)$ & $\mathrm{C} 1 \mathrm{~B}-\mathrm{P} 1 \mathrm{~B}-\mathrm{C} 15 \mathrm{~B}-\mathrm{C} 19 \mathrm{~B}$ & $9.8(5)$ \\
\hline $\mathrm{C} 1 \mathrm{~A}-\mathrm{P} 1 \mathrm{~A}-\mathrm{C} 34 \mathrm{~A}-\mathrm{C} 35 \mathrm{~A}$ & $-75.6(5)$ & $\mathrm{C} 1 \mathrm{~B}-\mathrm{P} 1 \mathrm{~B}-\mathrm{C} 34 \mathrm{~B}-\mathrm{C} 35 \mathrm{~B}$ & $-77.1(5)$ \\
\hline $\mathrm{C} 1 \mathrm{~A}-\mathrm{P} 1 \mathrm{~A}-\mathrm{C} 34 \mathrm{~A}-\mathrm{C} 39 \mathrm{~A}$ & $105.2(4)$ & $\mathrm{C} 1 \mathrm{~B}-\mathrm{P} 1 \mathrm{~B}-\mathrm{C} 34 \mathrm{~B}-\mathrm{C} 39 \mathrm{~B}$ & $101.5(5)$ \\
\hline $\mathrm{C} 1 \mathrm{~A}-\mathrm{C} 2 \mathrm{~A}-\mathrm{C} 3 \mathrm{~A}-\mathrm{Fe} 1 \mathrm{~A}$ & $-58.4(4)$ & $\mathrm{C} 1 \mathrm{~B}-\mathrm{C} 2 \mathrm{~B}-\mathrm{C} 3 \mathrm{~B}-\mathrm{Fe} 1 \mathrm{~B}$ & $-57.7(4)$ \\
\hline $\mathrm{C} 1 \mathrm{~A}-\mathrm{C} 2 \mathrm{~A}-\mathrm{C} 3 \mathrm{~A}-\mathrm{C} 4 \mathrm{~A}$ & $0.9(7)$ & $\mathrm{C} 1 \mathrm{~B}-\mathrm{C} 2 \mathrm{~B}-\mathrm{C} 3 \mathrm{~B}-\mathrm{C} 4 \mathrm{~B}$ & $0.0(7)$ \\
\hline $\mathrm{C} 1 \mathrm{~A}-\mathrm{C} 2 \mathrm{~A}-\mathrm{C} 11 \mathrm{~A}-\mathrm{C} 12 \mathrm{~A}$ & $173.3(8)$ & $\mathrm{C} 1 \mathrm{~B}-\mathrm{C} 2 \mathrm{~B}-\mathrm{C} 11 \mathrm{~B}-\mathrm{C} 12 \mathrm{~B}$ & $-155.1(7)$ \\
\hline $\mathrm{C} 2 \mathrm{~A}-\mathrm{C} 1 \mathrm{~A}-\mathrm{C} 5 \mathrm{~A}-\mathrm{Fe} 1 \mathrm{~A}$ & $59.6(4)$ & $\mathrm{C} 2 \mathrm{~B}-\mathrm{C} 1 \mathrm{~B}-\mathrm{C} 5 \mathrm{~B}-\mathrm{Fe} 1 \mathrm{~B}$ & $60.3(4)$ \\
\hline $\mathrm{C} 2 \mathrm{~A}-\mathrm{C} 1 \mathrm{~A}-\mathrm{C} 5 \mathrm{~A}-\mathrm{C} 4 \mathrm{~A}$ & $0.0(6)$ & $\mathrm{C} 2 \mathrm{~B}-\mathrm{C} 1 \mathrm{~B}-\mathrm{C} 5 \mathrm{~B}-\mathrm{C} 4 \mathrm{~B}$ & $1.0(7)$ \\
\hline $\mathrm{C} 2 \mathrm{~A}-\mathrm{C} 3 \mathrm{~A}-\mathrm{C} 4 \mathrm{~A}-\mathrm{Fe} 1 \mathrm{~A}$ & $-58.9(4)$ & $\mathrm{C} 2 \mathrm{~B}-\mathrm{C} 3 \mathrm{~B}-\mathrm{C} 4 \mathrm{~B}-\mathrm{Fe} 1 \mathrm{~B}$ & $-58.4(4)$ \\
\hline $\mathrm{C} 2 \mathrm{~A}-\mathrm{C} 3 \mathrm{~A}-\mathrm{C} 4 \mathrm{~A}-\mathrm{C} 5 \mathrm{~A}$ & $-1.0(7)$ & $\mathrm{C} 2 \mathrm{~B}-\mathrm{C} 3 \mathrm{~B}-\mathrm{C} 4 \mathrm{~B}-\mathrm{C} 5 \mathrm{~B}$ & $0.6(7)$ \\
\hline $\mathrm{C} 3 \mathrm{~A}-\mathrm{C} 2 \mathrm{~A}-\mathrm{C} 11 \mathrm{~A}-\mathrm{C} 12 \mathrm{~A}$ & $0.3(12)$ & $\mathrm{C} 3 \mathrm{~B}-\mathrm{C} 2 \mathrm{~B}-\mathrm{C} 11 \mathrm{~B}-\mathrm{C} 12 \mathrm{~B}$ & $24.8(11)$ \\
\hline $\mathrm{C} 3 \mathrm{~A}-\mathrm{C} 4 \mathrm{~A}-\mathrm{C} 5 \mathrm{~A}-\mathrm{Fe} 1 \mathrm{~A}$ & $-58.0(4)$ & $\mathrm{C} 3 \mathrm{~B}-\mathrm{C} 4 \mathrm{~B}-\mathrm{C} 5 \mathrm{~B}-\mathrm{Fe} 1 \mathrm{~B}$ & $-59.9(5)$ \\
\hline $\mathrm{C} 3 \mathrm{~A}-\mathrm{C} 4 \mathrm{~A}-\mathrm{C} 5 \mathrm{~A}-\mathrm{C} 1 \mathrm{~A}$ & $0.6(6)$ & $\mathrm{C} 3 \mathrm{~B}-\mathrm{C} 4 \mathrm{~B}-\mathrm{C} 5 \mathrm{~B}-\mathrm{C} 1 \mathrm{~B}$ & $-1.0(7)$ \\
\hline $\mathrm{C} 5 \mathrm{~A}-\mathrm{C} 1 \mathrm{~A}-\mathrm{C} 2 \mathrm{~A}-\mathrm{Fe} 1 \mathrm{~A}$ & $-59.5(4)$ & $\mathrm{C} 5 \mathrm{~B}-\mathrm{C} 1 \mathrm{~B}-\mathrm{C} 2 \mathrm{~B}-\mathrm{Fe} 1 \mathrm{~B}$ & $-59.6(4)$ \\
\hline $\mathrm{C} 5 \mathrm{~A}-\mathrm{C} 1 \mathrm{~A}-\mathrm{C} 2 \mathrm{~A}-\mathrm{C} 3 \mathrm{~A}$ & $-0.6(6)$ & $\mathrm{C} 5 \mathrm{~B}-\mathrm{C} 1 \mathrm{~B}-\mathrm{C} 2 \mathrm{~B}-\mathrm{C} 3 \mathrm{~B}$ & $-0.6(7)$ \\
\hline $\mathrm{C} 5 \mathrm{~A}-\mathrm{C} 1 \mathrm{~A}-\mathrm{C} 2 \mathrm{~A}-\mathrm{C} 11 \mathrm{~A}$ & $-174.6(6)$ & $\mathrm{C} 5 \mathrm{~B}-\mathrm{C} 1 \mathrm{~B}-\mathrm{C} 2 \mathrm{~B}-\mathrm{C} 11 \mathrm{~B}$ & $179.3(6)$ \\
\hline $\mathrm{C} 6 \mathrm{~A}-\mathrm{C} 7 \mathrm{~A}-\mathrm{C} 8 \mathrm{~A}-\mathrm{Fe} 1 \mathrm{~A}$ & $59.1(4)$ & $\mathrm{C} 6 \mathrm{~B}-\mathrm{C} 7 \mathrm{~B}-\mathrm{C} 8 \mathrm{~B}-\mathrm{Fe} 1 \mathrm{~B}$ & $60.2(5)$ \\
\hline $\mathrm{C} 6 \mathrm{~A}-\mathrm{C} 7 \mathrm{~A}-\mathrm{C} 8 \mathrm{~A}-\mathrm{C} 9 \mathrm{~A}$ & $-0.3(7)$ & $\mathrm{C} 6 \mathrm{~B}-\mathrm{C} 7 \mathrm{~B}-\mathrm{C} 8 \mathrm{~B}-\mathrm{C} 9 \mathrm{~B}$ & $0.2(8)$ \\
\hline $\mathrm{C} 7 \mathrm{~A}-\mathrm{C} 6 \mathrm{~A}-\mathrm{C} 10 \mathrm{~A}-\mathrm{Fe} 1 \mathrm{~A}$ & $-59.2(4)$ & $\mathrm{C} 7 \mathrm{~B}-\mathrm{C} 6 \mathrm{~B}-\mathrm{C} 10 \mathrm{~B}-\mathrm{Fe} 1 \mathrm{~B}$ & $-59.3(5)$ \\
\hline $\mathrm{C} 7 \mathrm{~A}-\mathrm{C} 6 \mathrm{~A}-\mathrm{C} 10 \mathrm{~A}-\mathrm{C} 9 \mathrm{~A}$ & $0.2(7)$ & $\mathrm{C} 7 \mathrm{~B}-\mathrm{C} 6 \mathrm{~B}-\mathrm{C} 10 \mathrm{~B}-\mathrm{C} 9 \mathrm{~B}$ & $-0.1(9)$ \\
\hline $\mathrm{C} 7 \mathrm{~A}-\mathrm{C} 8 \mathrm{~A}-\mathrm{C} 9 \mathrm{~A}-\mathrm{Fe} 1 \mathrm{~A}$ & $59.7(4)$ & $\mathrm{C} 7 \mathrm{~B}-\mathrm{C} 8 \mathrm{~B}-\mathrm{C} 9 \mathrm{~B}-\mathrm{Fe} 1 \mathrm{~B}$ & $59.2(5)$ \\
\hline $\mathrm{C} 7 \mathrm{~A}-\mathrm{C} 8 \mathrm{~A}-\mathrm{C} 9 \mathrm{~A}-\mathrm{C} 10 \mathrm{~A}$ & $0.4(7)$ & $\mathrm{C} 7 \mathrm{~B}-\mathrm{C} 8 \mathrm{~B}-\mathrm{C} 9 \mathrm{~B}-\mathrm{C} 10 \mathrm{~B}$ & $-0.3(9)$ \\
\hline $\mathrm{C} 8 \mathrm{~A}-\mathrm{C} 9 \mathrm{~A}-\mathrm{C} 10 \mathrm{~A}-\mathrm{Fe} 1 \mathrm{~A}$ & $59.4(4)$ & $\mathrm{C} 8 \mathrm{~B}-\mathrm{C} 9 \mathrm{~B}-\mathrm{C} 10 \mathrm{~B}-\mathrm{Fe} 1 \mathrm{~B}$ & $58.7(6)$ \\
\hline C8A-C9A-C10A-C6A & $-0.3(7)$ & $\mathrm{C} 8 \mathrm{~B}-\mathrm{C} 9 \mathrm{~B}-\mathrm{C} 10 \mathrm{~B}-\mathrm{C} 6 \mathrm{~B}$ & $0.2(9)$ \\
\hline $\mathrm{C} 10 \mathrm{~A}-\mathrm{C} 6 \mathrm{~A}-\mathrm{C} 7 \mathrm{~A}-\mathrm{Fe} 1 \mathrm{~A}$ & $58.9(4)$ & $\mathrm{C} 10 \mathrm{~B}-\mathrm{C} 6 \mathrm{~B}-\mathrm{C} 7 \mathrm{~B}-\mathrm{Fe} 1 \mathrm{~B}$ & $59.6(6)$ \\
\hline $\mathrm{C} 10 \mathrm{~A}-\mathrm{C} 6 \mathrm{~A}-\mathrm{C} 7 \mathrm{~A}-\mathrm{C} 8 \mathrm{~A}$ & $0.1(7)$ & $\mathrm{C} 10 \mathrm{~B}-\mathrm{C} 6 \mathrm{~B}-\mathrm{C} 7 \mathrm{~B}-\mathrm{C} 8 \mathrm{~B}$ & $-0.1(8)$ \\
\hline $\mathrm{C} 11 \mathrm{~A}-\mathrm{C} 2 \mathrm{~A}-\mathrm{C} 3 \mathrm{~A}-\mathrm{Fe} 1 \mathrm{~A}$ & $115.8(6)$ & $\mathrm{C} 11 \mathrm{~B}-\mathrm{C} 2 \mathrm{~B}-\mathrm{C} 3 \mathrm{~B}-\mathrm{Fe} 1 \mathrm{~B}$ & $122.4(6)$ \\
\hline $\mathrm{C} 11 \mathrm{~A}-\mathrm{C} 2 \mathrm{~A}-\mathrm{C} 3 \mathrm{~A}-\mathrm{C} 4 \mathrm{~A}$ & $175.2(6)$ & $\mathrm{C} 11 \mathrm{~B}-\mathrm{C} 2 \mathrm{~B}-\mathrm{C} 3 \mathrm{~B}-\mathrm{C} 4 \mathrm{~B}$ & $-179.9(6)$ \\
\hline $\mathrm{C} 15 \mathrm{~A}-\mathrm{P} 1 \mathrm{~A}-\mathrm{C} 1 \mathrm{~A}-\mathrm{Fe} 1 \mathrm{~A}$ & $-172.6(3)$ & $\mathrm{C} 15 \mathrm{~B}-\mathrm{P} 1 \mathrm{~B}-\mathrm{C} 1 \mathrm{~B}-\mathrm{Fe} 1 \mathrm{~B}$ & $-172.4(3)$ \\
\hline $\mathrm{C} 15 \mathrm{~A}-\mathrm{P} 1 \mathrm{~A}-\mathrm{C} 1 \mathrm{~A}-\mathrm{C} 2 \mathrm{~A}$ & $93.7(5)$ & $\mathrm{C} 15 \mathrm{~B}-\mathrm{P} 1 \mathrm{~B}-\mathrm{C} 1 \mathrm{~B}-\mathrm{C} 2 \mathrm{~B}$ & $93.4(5)$ \\
\hline
\end{tabular}




\begin{tabular}{|c|c|}
\hline $\mathrm{C} 15 \mathrm{~A}-\mathrm{P} 1 \mathrm{~A}-\mathrm{C} 1 \mathrm{~A}-\mathrm{C} 5 \mathrm{~A}$ & $-82.5(5)$ \\
\hline $\mathrm{C} 15 \mathrm{~A}-\mathrm{P} 1 \mathrm{~A}-\mathrm{C} 34 \mathrm{~A}-\mathrm{C} 35 \mathrm{~A}$ & $31.8(5)$ \\
\hline $\mathrm{C} 15 \mathrm{~A}-\mathrm{P} 1 \mathrm{~A}-\mathrm{C} 34 \mathrm{~A}-\mathrm{C} 39 \mathrm{~A}$ & $-147.4(4)$ \\
\hline $\mathrm{C} 15 \mathrm{~A}-\mathrm{C} 16 \mathrm{~A}-\mathrm{C} 17 \mathrm{~A}-\mathrm{Fe} 2 \mathrm{~A}$ & $-59.4(3)$ \\
\hline $\mathrm{C} 15 \mathrm{~A}-\mathrm{C} 16 \mathrm{~A}-\mathrm{C} 17 \mathrm{~A}-\mathrm{C} 18 \mathrm{~A}$ & $-0.1(6)$ \\
\hline $\mathrm{C} 15 \mathrm{~A}-\mathrm{C} 16 \mathrm{~A}-\mathrm{C} 25 \mathrm{~A}-\mathrm{O} 1 \mathrm{~A}$ & $-81.6(7)$ \\
\hline $\mathrm{C} 15 \mathrm{~A}-\mathrm{C} 16 \mathrm{~A}-\mathrm{C} 25 \mathrm{~A}-\mathrm{C} 26 \mathrm{~A}$ & $157.0(6)$ \\
\hline $\mathrm{C} 16 \mathrm{~A}-\mathrm{C} 15 \mathrm{~A}-\mathrm{C} 19 \mathrm{~A}-\mathrm{Fe} 2 \mathrm{~A}$ & $59.1(3)$ \\
\hline $\mathrm{C} 16 \mathrm{~A}-\mathrm{C} 15 \mathrm{~A}-\mathrm{C} 19 \mathrm{~A}-\mathrm{C} 18 \mathrm{~A}$ & $-0.7(6)$ \\
\hline $\mathrm{C} 16 \mathrm{~A}-\mathrm{C} 17 \mathrm{~A}-\mathrm{C} 18 \mathrm{~A}-\mathrm{Fe} 2 \mathrm{~A}$ & $-58.4(4)$ \\
\hline $\mathrm{C} 16 \mathrm{~A}-\mathrm{C} 17 \mathrm{~A}-\mathrm{C} 18 \mathrm{~A}-\mathrm{C} 19 \mathrm{~A}$ & $-0.3(7)$ \\
\hline $\mathrm{C} 17 \mathrm{~A}-\mathrm{C} 16 \mathrm{~A}-\mathrm{C} 25 \mathrm{~A}-\mathrm{O} 1 \mathrm{~A}$ & $92.2(6)$ \\
\hline $\mathrm{C} 17 \mathrm{~A}-\mathrm{C} 16 \mathrm{~A}-\mathrm{C} 25 \mathrm{~A}-\mathrm{C} 26 \mathrm{~A}$ & $-29.2(9)$ \\
\hline $\mathrm{C} 17 \mathrm{~A}-\mathrm{C} 18 \mathrm{~A}-\mathrm{C} 19 \mathrm{~A}-\mathrm{Fe} 2 \mathrm{~A}$ & $-58.1(4)$ \\
\hline $\mathrm{C} 17 \mathrm{~A}-\mathrm{C} 18 \mathrm{~A}-\mathrm{C} 19 \mathrm{~A}-\mathrm{C} 15 \mathrm{~A}$ & $0.6(7)$ \\
\hline $\mathrm{C} 19 \mathrm{~A}-\mathrm{C} 15 \mathrm{~A}-\mathrm{C} 16 \mathrm{~A}-\mathrm{Fe} 2 \mathrm{~A}$ & $-59.4(4)$ \\
\hline $\mathrm{C} 19 \mathrm{~A}-\mathrm{C} 15 \mathrm{~A}-\mathrm{C} 16 \mathrm{~A}-\mathrm{C} 17 \mathrm{~A}$ & $0.5(6)$ \\
\hline $\mathrm{C} 19 \mathrm{~A}-\mathrm{C} 15 \mathrm{~A}-\mathrm{C} 16 \mathrm{~A}-\mathrm{C} 25 \mathrm{~A}$ & $175.2(5)$ \\
\hline $\mathrm{C} 20 \mathrm{~A}-\mathrm{C} 21 \mathrm{~A}-\mathrm{C} 22 \mathrm{~A}-\mathrm{Fe} 2 \mathrm{~A}$ & $60.1(5)$ \\
\hline $\mathrm{C} 20 \mathrm{~A}-\mathrm{C} 21 \mathrm{~A}-\mathrm{C} 22 \mathrm{~A}-\mathrm{C} 23 \mathrm{~A}$ & $0.2(8)$ \\
\hline $\mathrm{C} 21 \mathrm{~A}-\mathrm{C} 20 \mathrm{~A}-\mathrm{C} 24 \mathrm{~A}-\mathrm{Fe} 2 \mathrm{~A}$ & $-58.6(5)$ \\
\hline $\mathrm{C} 21 \mathrm{~A}-\mathrm{C} 20 \mathrm{~A}-\mathrm{C} 24 \mathrm{~A}-\mathrm{C} 23 \mathrm{~A}$ & $0.4(8)$ \\
\hline $\mathrm{C} 21 \mathrm{~A}-\mathrm{C} 22 \mathrm{~A}-\mathrm{C} 23 \mathrm{~A}-\mathrm{Fe} 2 \mathrm{~A}$ & $60.3(5)$ \\
\hline $\mathrm{C} 21 \mathrm{~A}-\mathrm{C} 22 \mathrm{~A}-\mathrm{C} 23 \mathrm{~A}-\mathrm{C} 24 \mathrm{~A}$ & $0.1(8)$ \\
\hline $\mathrm{C} 22 \mathrm{~A}-\mathrm{C} 23 \mathrm{~A}-\mathrm{C} 24 \mathrm{~A}-\mathrm{Fe} 2 \mathrm{~A}$ & $59.5(5)$ \\
\hline $\mathrm{C} 22 \mathrm{~A}-\mathrm{C} 23 \mathrm{~A}-\mathrm{C} 24 \mathrm{~A}-\mathrm{C} 20 \mathrm{~A}$ & $-0.3(8)$ \\
\hline $\mathrm{C} 24 \mathrm{~A}-\mathrm{C} 20 \mathrm{~A}-\mathrm{C} 21 \mathrm{~A}-\mathrm{Fe} 2 \mathrm{~A}$ & $59.0(5)$ \\
\hline $\mathrm{C} 24 \mathrm{~A}-\mathrm{C} 20 \mathrm{~A}-\mathrm{C} 21 \mathrm{~A}-\mathrm{C} 22 \mathrm{~A}$ & $-0.4(8)$ \\
\hline $\mathrm{C} 25 \mathrm{~A}-\mathrm{C} 16 \mathrm{~A}-\mathrm{C} 17 \mathrm{~A}-\mathrm{Fe} 2 \mathrm{~A}$ & $125.8(5)$ \\
\hline $\mathrm{C} 25 \mathrm{~A}-\mathrm{C} 16 \mathrm{~A}-\mathrm{C} 17 \mathrm{~A}-\mathrm{C} 18 \mathrm{~A}$ & $-174.9(5)$ \\
\hline $\mathrm{C} 34 \mathrm{~A}-\mathrm{P} 1 \mathrm{~A}-\mathrm{C} 1 \mathrm{~A}-\mathrm{Fe} 1 \mathrm{~A}$ & $-64.6(4)$ \\
\hline $\mathrm{C} 34 \mathrm{~A}-\mathrm{P} 1 \mathrm{~A}-\mathrm{C} 1 \mathrm{~A}-\mathrm{C} 2 \mathrm{~A}$ & $-158.4(5)$ \\
\hline $\mathrm{C} 34 \mathrm{~A}-\mathrm{P} 1 \mathrm{~A}-\mathrm{C} 1 \mathrm{~A}-\mathrm{C} 5 \mathrm{~A}$ & $25.4(5)$ \\
\hline $\mathrm{C} 34 \mathrm{~A}-\mathrm{P} 1 \mathrm{~A}-\mathrm{C} 15 \mathrm{~A}-\mathrm{Fe} 2 \mathrm{~A}$ & $167.2(3)$ \\
\hline $\mathrm{C} 34 \mathrm{~A}-\mathrm{P} 1 \mathrm{~A}-\mathrm{C} 15 \mathrm{~A}-\mathrm{C} 16 \mathrm{~A}$ & $72.8(5)$ \\
\hline $\mathrm{C} 34 \mathrm{~A}-\mathrm{P} 1 \mathrm{~A}-\mathrm{C} 15 \mathrm{~A}-\mathrm{C} 19 \mathrm{~A}$ & $-101.1(5)$ \\
\hline $\mathrm{C} 34 \mathrm{~A}-\mathrm{C} 35 \mathrm{~A}-\mathrm{C} 36 \mathrm{~A}-\mathrm{C} 37 \mathrm{~A}$ & $0.4(9)$ \\
\hline $\mathrm{C} 35 \mathrm{~A}-\mathrm{C} 34 \mathrm{~A}-\mathrm{C} 39 \mathrm{~A}-\mathrm{C} 38 \mathrm{~A}$ & $0.2(8)$ \\
\hline $\mathrm{C} 35 \mathrm{~A}-\mathrm{C} 36 \mathrm{~A}-\mathrm{C} 37 \mathrm{~A}-\mathrm{C} 38 \mathrm{~A}$ & $-0.1(9)$ \\
\hline $\mathrm{C} 36 \mathrm{~A}-\mathrm{C} 37 \mathrm{~A}-\mathrm{C} 38 \mathrm{~A}-\mathrm{C} 39 \mathrm{~A}$ & $-0.1(10)$ \\
\hline $\mathrm{C} 37 \mathrm{~A}-\mathrm{C} 38 \mathrm{~A}-\mathrm{C} 39 \mathrm{~A}-\mathrm{C} 34 \mathrm{~A}$ & $0.1(9)$ \\
\hline $\mathrm{C} 39 \mathrm{~A}-\mathrm{C} 34 \mathrm{~A}-\mathrm{C} 35 \mathrm{~A}-\mathrm{C} 36 \mathrm{~A}$ & $-0.5(8)$ \\
\hline
\end{tabular}

\begin{tabular}{|c|c|}
\hline $\mathrm{C} 15 \mathrm{~B}-\mathrm{P} 1 \mathrm{~B}-\mathrm{C} 1 \mathrm{~B}-\mathrm{C} 5 \mathrm{~B}$ & $-84.6(5)$ \\
\hline $\mathrm{C} 15 \mathrm{~B}-\mathrm{P} 1 \mathrm{~B}-\mathrm{C} 34 \mathrm{~B}-\mathrm{C} 35 \mathrm{~B}$ & $30.8(5)$ \\
\hline $\mathrm{C} 15 \mathrm{~B}-\mathrm{P} 1 \mathrm{~B}-\mathrm{C} 34 \mathrm{~B}-\mathrm{C} 39 \mathrm{~B}$ & $-150.5(4)$ \\
\hline $\mathrm{C} 15 \mathrm{~B}-\mathrm{C} 16 \mathrm{~B}-\mathrm{C} 17 \mathrm{~B}-\mathrm{Fe} 2 \mathrm{~B}$ & $-58.8(4)$ \\
\hline $\mathrm{C} 15 \mathrm{~B}-\mathrm{C} 16 \mathrm{~B}-\mathrm{C} 17 \mathrm{~B}-\mathrm{C} 18 \mathrm{~B}$ & $0.3(8)$ \\
\hline $\mathrm{C} 15 \mathrm{~B}-\mathrm{C} 16 \mathrm{~B}-\mathrm{C} 25 \mathrm{~B}-\mathrm{O} 1 \mathrm{~B}$ & $-84.6(8)$ \\
\hline $\mathrm{C} 15 \mathrm{~B}-\mathrm{C} 16 \mathrm{~B}-\mathrm{C} 25 \mathrm{~B}-\mathrm{C} 26 \mathrm{~B}$ & $159.4(7)$ \\
\hline $\mathrm{C} 16 \mathrm{~B}-\mathrm{C} 15 \mathrm{~B}-\mathrm{C} 19 \mathrm{~B}-\mathrm{Fe} 2 \mathrm{~B}$ & $59.9(4)$ \\
\hline $\mathrm{C} 16 \mathrm{~B}-\mathrm{C} 15 \mathrm{~B}-\mathrm{C} 19 \mathrm{~B}-\mathrm{C} 18 \mathrm{~B}$ & $0.7(7)$ \\
\hline $\mathrm{C} 16 \mathrm{~B}-\mathrm{C} 17 \mathrm{~B}-\mathrm{C} 18 \mathrm{~B}-\mathrm{Fe} 2 \mathrm{~B}$ & $-58.4(5)$ \\
\hline $\mathrm{C} 16 \mathrm{~B}-\mathrm{C} 17 \mathrm{~B}-\mathrm{C} 18 \mathrm{~B}-\mathrm{C} 19 \mathrm{~B}$ & $0.1(8)$ \\
\hline $\mathrm{C} 17 \mathrm{~B}-\mathrm{C} 16 \mathrm{~B}-\mathrm{C} 25 \mathrm{~B}-\mathrm{O} 1 \mathrm{~B}$ & $87.6(11)$ \\
\hline $\mathrm{C} 17 \mathrm{~B}-\mathrm{C} 16 \mathrm{~B}-\mathrm{C} 25 \mathrm{~B}-\mathrm{C} 26 \mathrm{~B}$ & $-28.4(14)$ \\
\hline $\mathrm{C} 17 \mathrm{~B}-\mathrm{C} 18 \mathrm{~B}-\mathrm{C} 19 \mathrm{~B}-\mathrm{Fe} 2 \mathrm{~B}$ & $-58.7(5)$ \\
\hline $\mathrm{C} 17 \mathrm{~B}-\mathrm{C} 18 \mathrm{~B}-\mathrm{C} 19 \mathrm{~B}-\mathrm{C} 15 \mathrm{~B}$ & $-0.5(7)$ \\
\hline $\mathrm{C} 19 \mathrm{~B}-\mathrm{C} 15 \mathrm{~B}-\mathrm{C} 16 \mathrm{~B}-\mathrm{Fe} 2 \mathrm{~B}$ & $-60.1(4)$ \\
\hline $\mathrm{C} 19 \mathrm{~B}-\mathrm{C} 15 \mathrm{~B}-\mathrm{C} 16 \mathrm{~B}-\mathrm{C} 17 \mathrm{~B}$ & $-0.6(7)$ \\
\hline $\mathrm{C} 19 \mathrm{~B}-\mathrm{C} 15 \mathrm{~B}-\mathrm{C} 16 \mathrm{~B}-\mathrm{C} 25 \mathrm{~B}$ & $173.1(8)$ \\
\hline $\mathrm{C} 20 \mathrm{~B}-\mathrm{C} 21 \mathrm{~B}-\mathrm{C} 22 \mathrm{~B}-\mathrm{Fe} 2 \mathrm{~B}$ & $59.7(6)$ \\
\hline $\mathrm{C} 20 \mathrm{~B}-\mathrm{C} 21 \mathrm{~B}-\mathrm{C} 22 \mathrm{~B}-\mathrm{C} 23 \mathrm{~B}$ & $-0.2(10)$ \\
\hline $\mathrm{C} 21 \mathrm{~B}-\mathrm{C} 20 \mathrm{~B}-\mathrm{C} 24 \mathrm{~B}-\mathrm{Fe} 2 \mathrm{~B}$ & $-59.9(5)$ \\
\hline $\mathrm{C} 21 \mathrm{~B}-\mathrm{C} 20 \mathrm{~B}-\mathrm{C} 24 \mathrm{~B}-\mathrm{C} 23 \mathrm{~B}$ & $-0.9(8)$ \\
\hline $\mathrm{C} 21 \mathrm{~B}-\mathrm{C} 22 \mathrm{~B}-\mathrm{C} 23 \mathrm{~B}-\mathrm{Fe} 2 \mathrm{~B}$ & $59.9(7)$ \\
\hline $\mathrm{C} 21 \mathrm{~B}-\mathrm{C} 22 \mathrm{~B}-\mathrm{C} 23 \mathrm{~B}-\mathrm{C} 24 \mathrm{~B}$ & $-0.4(11)$ \\
\hline $\mathrm{C} 22 \mathrm{~B}-\mathrm{C} 23 \mathrm{~B}-\mathrm{C} 24 \mathrm{~B}-\mathrm{Fe} 2 \mathrm{~B}$ & $60.2(7)$ \\
\hline $\mathrm{C} 22 \mathrm{~B}-\mathrm{C} 23 \mathrm{~B}-\mathrm{C} 24 \mathrm{~B}-\mathrm{C} 20 \mathrm{~B}$ & $0.8(10)$ \\
\hline $\mathrm{C} 24 \mathrm{~B}-\mathrm{C} 20 \mathrm{~B}-\mathrm{C} 21 \mathrm{~B}-\mathrm{Fe} 2 \mathrm{~B}$ & $60.4(5)$ \\
\hline $\mathrm{C} 24 \mathrm{~B}-\mathrm{C} 20 \mathrm{~B}-\mathrm{C} 21 \mathrm{~B}-\mathrm{C} 22 \mathrm{~B}$ & $0.7(9)$ \\
\hline $\mathrm{C} 25 \mathrm{~B}-\mathrm{C} 16 \mathrm{~B}-\mathrm{C} 17 \mathrm{~B}-\mathrm{Fe} 2 \mathrm{~B}$ & $127.9(10)$ \\
\hline $\mathrm{C} 25 \mathrm{~B}-\mathrm{C} 16 \mathrm{~B}-\mathrm{C} 17 \mathrm{~B}-\mathrm{C} 18 \mathrm{~B}$ & $-173.0(9)$ \\
\hline C34B-P1B-C1B-Fe1B & $-66.2(4)$ \\
\hline $\mathrm{C} 34 \mathrm{~B}-\mathrm{P} 1 \mathrm{~B}-\mathrm{C} 1 \mathrm{~B}-\mathrm{C} 2 \mathrm{~B}$ & $-160.4(5)$ \\
\hline $\mathrm{C} 34 \mathrm{~B}-\mathrm{P} 1 \mathrm{~B}-\mathrm{C} 1 \mathrm{~B}-\mathrm{C} 5 \mathrm{~B}$ & $21.6(5)$ \\
\hline $\mathrm{C} 34 \mathrm{~B}-\mathrm{P} 1 \mathrm{~B}-\mathrm{C} 15 \mathrm{~B}-\mathrm{Fe} 2 \mathrm{~B}$ & $168.2(4)$ \\
\hline $\mathrm{C} 34 \mathrm{~B}-\mathrm{P} 1 \mathrm{~B}-\mathrm{C} 15 \mathrm{~B}-\mathrm{C} 16 \mathrm{~B}$ & $71.6(6)$ \\
\hline $\mathrm{C} 34 \mathrm{~B}-\mathrm{P} 1 \mathrm{~B}-\mathrm{C} 15 \mathrm{~B}-\mathrm{C} 19 \mathrm{~B}$ & $-97.5(5)$ \\
\hline $\mathrm{C} 34 \mathrm{~B}-\mathrm{C} 35 \mathrm{~B}-\mathrm{C} 36 \mathrm{~B}-\mathrm{C} 37 \mathrm{~B}$ & $-1.3(10)$ \\
\hline $\mathrm{C} 35 \mathrm{~B}-\mathrm{C} 34 \mathrm{~B}-\mathrm{C} 39 \mathrm{~B}-\mathrm{C} 38 \mathrm{~B}$ & $1.1(9)$ \\
\hline $\mathrm{C} 35 \mathrm{~B}-\mathrm{C} 36 \mathrm{~B}-\mathrm{C} 37 \mathrm{~B}-\mathrm{C} 38 \mathrm{~B}$ & $1.8(11)$ \\
\hline $\mathrm{C} 36 \mathrm{~B}-\mathrm{C} 37 \mathrm{~B}-\mathrm{C} 38 \mathrm{~B}-\mathrm{C} 39 \mathrm{~B}$ & $-0.8(11)$ \\
\hline $\mathrm{C} 37 \mathrm{~B}-\mathrm{C} 38 \mathrm{~B}-\mathrm{C} 39 \mathrm{~B}-\mathrm{C} 34 \mathrm{~B}$ & $-0.6(10)$ \\
\hline $\mathrm{C} 39 \mathrm{~B}-\mathrm{C} 34 \mathrm{~B}-\mathrm{C} 35 \mathrm{~B}-\mathrm{C} 36 \mathrm{~B}$ & $-0.1(9)$ \\
\hline
\end{tabular}


$\left\{\left(2 S_{\mathrm{p}}\right)-2-\left[(1 R)-1-(\right.\right.$ Acetyloxy)ethyl] ferrocen-1-yl $\}\left\{\left(2 S_{\mathrm{p}}\right)-2-[(1 R)-1\right.$-hydroxyethyl] ferrocen-1-yl $\}$ phenyl-( $\left.R\right)$ -

phosphane sulfide (8c)

Crystal data

$\left[\mathrm{Fe}_{2}\left(\mathrm{C}_{5} \mathrm{H}_{5}\right)_{2}\left(\mathrm{C}_{22} \mathrm{H}_{23} \mathrm{O}_{3} \mathrm{PS}\right)\right]$

$M_{r}=640.31$

Orthorhombic, $P 2_{1} 2_{1} 2_{1}$

$a=7.8204$ (11) $\AA$

$b=17.835(3) \AA$

$c=20.394(2) \AA$

$V=2844.5(7) \AA^{3}$

$Z=4$

$F(000)=1328$

Data collection

Bruker X8 APEXII

diffractometer

Radiation source: sealed xray tube, Incoatec IuS

$\varphi$ and $\omega$ scans

Absorption correction: multi-scan

(SADABS; Bruker, 2008)

$T_{\min }=0.568, T_{\max }=0.745$

35430 measured reflections

\section{Refinement}

Refinement on $F^{2}$

Least-squares matrix: full

$R\left[F^{2}>2 \sigma\left(F^{2}\right)\right]=0.054$

$w R\left(F^{2}\right)=0.093$

$S=1.02$

5258 reflections

356 parameters

0 restraints

Primary atom site location: structure-invariant direct methods
$D_{\mathrm{x}}=1.495 \mathrm{Mg} \mathrm{m}^{-3}$

Mo $K \alpha$ radiation, $\lambda=0.71073 \AA$

Cell parameters from 1636 reflections

$\theta=2.3-17.7^{\circ}$

$\mu=1.18 \mathrm{~mm}^{-1}$

$T=130 \mathrm{~K}$

Plate, clear orange

$0.1 \times 0.06 \times 0.01 \mathrm{~mm}$

5258 independent reflections

3417 reflections with $I>2 \sigma(I)$

$R_{\text {int }}=0.167$

$\theta_{\max }=25.5^{\circ}, \theta_{\min }=2.3^{\circ}$

$h=-9 \rightarrow 9$

$k=-21 \rightarrow 21$

$l=-20 \rightarrow 24$

Hydrogen site location: inferred from neighbouring sites

$\mathrm{H}$-atom parameters constrained

$w=1 /\left[\sigma^{2}\left(F_{\mathrm{o}}^{2}\right)+(0.0262 P)^{2}\right]$

where $P=\left(F_{\mathrm{o}}^{2}+2 F_{\mathrm{c}}^{2}\right) / 3$

$(\Delta / \sigma)_{\max }=0.001$

$\Delta \rho_{\max }=0.42 \mathrm{e} \AA^{-3}$

$\Delta \rho_{\min }=-0.41 \mathrm{e} \AA^{-3}$

Absolute structure: Flack $x$ determined using 1051 quotients $[(\mathrm{I}+)-(\mathrm{I}-)] /[(\mathrm{I}+)+(\mathrm{I}-)]$ (Parsons et al., 2013)

Absolute structure parameter: 0.00 (2)

Special details

Geometry. All esds (except the esd in the dihedral angle between two 1.s. planes) are estimated using the full covariance matrix. The cell esds are taken into account individually in the estimation of esds in distances, angles and torsion angles; correlations between esds in cell parameters are only used when they are defined by crystal symmetry. An approximate (isotropic) treatment of cell esds is used for estimating esds involving 1.s. planes.

Refinement._olex2_refinement_description

1. Fixed Uiso At 1.2 times of: All $\mathrm{C}(\mathrm{H})$ groups At 1.5 times of: $\mathrm{All} \mathrm{C}(\mathrm{H}, \mathrm{H}, \mathrm{H})$ groups, $\mathrm{All} \mathrm{O}(\mathrm{H})$ groups 2 .a Ternary $\mathrm{CH}$ refined with riding coordinates: $\mathrm{C} 11(\mathrm{H} 11), \mathrm{C} 25(\mathrm{H} 25)$ 2.b Aromatic/amide $\mathrm{H}$ refined with riding coordinates: $\mathrm{C} 3(\mathrm{H} 3 \mathrm{~A})$, C4(H4), C5(H5), C6(H6), C7(H7), C8(H8), C9(H9), C10(H10), C17(H17), C18(H18), C19(H19), C20(H20), C21(H21), C22(H22), C23(H23), C24(H24), C39(H39), C38(H38), C37(H37), C36(H36), C35(H35) 2.c Idealised Me refined as rotating group: C13(H13A,H13B,H13C), C14(H14A,H14B,H14C), C26(H26A,H26B,H26C) 2.d Idealised tetrahedral $\mathrm{OH}$ refined as rotating group: $\mathrm{O} 3(\mathrm{H} 3)$ 
Fractional atomic coordinates and isotropic or equivalent isotropic displacement parameters $\left(\AA^{2}\right)$

\begin{tabular}{|c|c|c|c|c|}
\hline & $x$ & $y$ & $z$ & $U_{\text {iso }} * / U_{\text {eq }}$ \\
\hline Fe1 & $0.49633(16)$ & $0.66812(6)$ & $0.63443(5)$ & $0.0260(3)$ \\
\hline $\mathrm{Fe} 2$ & $0.09126(14)$ & $0.49141(7)$ & $0.85171(5)$ & $0.0295(3)$ \\
\hline $\mathrm{S} 1$ & $-0.0032(3)$ & $0.68244(11)$ & $0.73939(9)$ & $0.0282(5)$ \\
\hline P1 & $0.2250(3)$ & $0.65243(12)$ & $0.76895(9)$ & $0.0201(5)$ \\
\hline $\mathrm{O} 1$ & $0.1576(7)$ & $0.4879(3)$ & $0.5949(2)$ & $0.0354(15)$ \\
\hline $\mathrm{O} 2$ & $-0.1257(9)$ & $0.4851(4)$ & $0.5812(4)$ & $0.076(2)$ \\
\hline $\mathrm{O} 3$ & $0.1577(10)$ & $0.7052(4)$ & 0.9575 & $0.064(2)$ \\
\hline H3 & 0.239200 & 0.718829 & 0.933439 & $0.096^{*}$ \\
\hline $\mathrm{C} 1$ & $0.3634(9)$ & $0.6151(4)$ & $0.7074(3)$ & $0.0175(18)$ \\
\hline $\mathrm{C} 2$ & $0.3235(10)$ & $0.5812(4)$ & $0.6444(4)$ & 0.0223 (19) \\
\hline C3 & $0.4802(11)$ & $0.5554(4)$ & $0.6176(3)$ & $0.029(2)$ \\
\hline $\mathrm{H} 3 \mathrm{~A}$ & 0.492286 & 0.531214 & 0.576413 & $0.034^{*}$ \\
\hline $\mathrm{C} 4$ & $0.6161(11)$ & $0.5711(4)$ & $0.6614(4)$ & $0.029(2)$ \\
\hline $\mathrm{H} 4$ & 0.733257 & 0.559028 & 0.655074 & $0.035^{*}$ \\
\hline $\mathrm{C} 5$ & $0.5444(9)$ & $0.6084(4)$ & $0.7166(3)$ & $0.024(2)$ \\
\hline H5 & 0.606541 & 0.626007 & 0.753536 & $0.029^{*}$ \\
\hline C6 & $0.4284(16)$ & $0.7782(6)$ & $0.6237(7)$ & 0.075 (4) \\
\hline H6 & 0.332502 & 0.801953 & 0.643394 & $0.090 *$ \\
\hline $\mathrm{C} 7$ & 0.4345 (19) & $0.7422(8)$ & $0.5627(7)$ & $0.085(5)$ \\
\hline $\mathrm{H} 7$ & 0.341397 & 0.737606 & 0.533030 & $0.102 *$ \\
\hline $\mathrm{C} 8$ & $0.597(2)$ & $0.7144(6)$ & $0.5525(5)$ & $0.065(3)$ \\
\hline H8 & 0.634698 & 0.687015 & 0.515338 & $0.077^{*}$ \\
\hline C9 & $0.6920(13)$ & $0.7334(5)$ & $0.6053(5)$ & $0.046(3)$ \\
\hline H9 & 0.809738 & 0.721973 & 0.610997 & $0.056^{*}$ \\
\hline $\mathrm{C} 10$ & $0.5926(16)$ & $0.7719(5)$ & $0.6495(5)$ & $0.050(3)$ \\
\hline H10 & 0.629610 & 0.790900 & 0.690615 & $0.060 *$ \\
\hline $\mathrm{C} 11$ & $0.1529(11)$ & $0.5671(5)$ & $0.6137(4)$ & $0.029(2)$ \\
\hline H11 & 0.061959 & 0.574419 & 0.647608 & $0.035^{*}$ \\
\hline $\mathrm{C} 12$ & $0.0085(15)$ & $0.4537(5)$ & $0.5805(4)$ & $0.047(3)$ \\
\hline $\mathrm{C} 13$ & $0.0325(14)$ & $0.3729(5)$ & $0.5656(5)$ & $0.072(4)$ \\
\hline H13A & 0.132793 & 0.366649 & 0.537278 & $0.108 *$ \\
\hline H13B & -0.069137 & 0.353707 & 0.543079 & $0.108^{*}$ \\
\hline $\mathrm{H} 13 \mathrm{C}$ & 0.049797 & 0.345138 & 0.606494 & $0.108 *$ \\
\hline C14 & 0.1115 (12) & $0.6151(5)$ & $0.5548(4)$ & $0.046(3)$ \\
\hline H14A & 0.201853 & 0.609504 & 0.521892 & $0.068^{*}$ \\
\hline H14B & 0.103807 & 0.667728 & 0.568276 & $0.068^{*}$ \\
\hline $\mathrm{H} 14 \mathrm{C}$ & 0.001983 & 0.599150 & 0.536085 & $0.068 *$ \\
\hline $\mathrm{C} 15$ & $0.2338(10)$ & $0.5832(4)$ & $0.8332(3)$ & $0.021(2)$ \\
\hline $\mathrm{C} 16$ & $0.1549(11)$ & 0.5889 & $0.8967(4)$ & $0.029(2)$ \\
\hline $\mathrm{C} 17$ & $0.2137(11)$ & $0.5271(5)$ & $0.9342(4)$ & $0.033(2)$ \\
\hline H17 & 0.183025 & 0.516780 & 0.978410 & $0.040 *$ \\
\hline $\mathrm{C} 18$ & $0.3254(10)$ & $0.4832(5)$ & 0.8954 & $0.027(2)$ \\
\hline H18 & 0.380377 & 0.438271 & 0.908827 & $0.033^{*}$ \\
\hline C19 & $0.3413(9)$ & $0.5175(4)$ & $0.8332(4)$ & $0.0236(19)$ \\
\hline H19 & 0.410138 & 0.500408 & 0.797835 & $0.028 *$ \\
\hline
\end{tabular}




$\begin{array}{lllll}\text { C20 } & -0.1633(11) & 0.4916(6) & 0.8299(6) & 0.060(3) \\ \text { H20 } & -0.237723 & 0.533607 & 0.832684 & 0.072^{*} \\ \text { C21 } & -0.0686(13) & 0.4712(6) & 0.7752(5) & 0.054(3) \\ \text { H21 } & -0.066901 & 0.496847 & 0.734343 & 0.064^{*} \\ \text { C22 } & 0.0249(14) & 0.4057(5) & 0.7906(5) & 0.061(3) \\ \text { H22 } & 0.099446 & 0.379034 & 0.762211 & 0.073^{*} \\ \text { C23 } & -0.0135(13) & 0.3878(5) & 0.8557(6) & 0.059(3) \\ \text { H23 } & 0.032814 & 0.346702 & 0.879414 & 0.071^{*} \\ \text { C24 } & -0.1310(12) & 0.4400(6) & 0.8807(5) & 0.056(3) \\ \text { H24 } & -0.179235 & 0.440467 & 0.923453 & 0.068^{*} \\ \text { C25 } & 0.0485(11) & 0.6538(5) & 0.9215(4) & 0.044(3) \\ \text { H25 } & -0.002628 & 0.680689 & 0.883175 & 0.053^{*} \\ \text { C26 } & -0.0922(13) & 0.6312(6) & 0.9678(4) & 0.060(3) \\ \text { H26A } & -0.043240 & 0.603747 & 1.004978 & 0.090^{*} \\ \text { H26B } & -0.174199 & 0.599062 & 0.944821 & 0.090^{*} \\ \text { H26C } & -0.150811 & 0.676181 & 0.983920 & 0.090^{*} \\ \text { C34 } & 0.3455(10) & 0.7300(4) & 0.8041(3) & 0.024(2) \\ \text { C39 } & 0.3041(11) & 0.8034(5) & 0.7878(4) & 0.033(2) \\ \text { H39 } & 0.209659 & 0.812782 & 0.759629 & 0.039^{*} \\ \text { C38 } & 0.3979(12) & 0.8626(5) & 0.8117(4) & 0.039(2) \\ \text { H38 } & 0.368464 & 0.912456 & 0.799876 & 0.047^{*} \\ \text { C37 } & 0.5340(12) & 0.8496(5) & 0.8527(4) & 0.045(3) \\ \text { H37 } & 0.600124 & 0.890575 & 0.868362 & 0.054^{*} \\ \text { C36 } & 0.5758(12) & 0.7764(5) & 0.8717(4) & 0.046(3) \\ \text { H36 } & 0.668270 & 0.767494 & 0.900850 & 0.056^{*} \\ \text { C35 } & 0.4802(11) & 0.7171(4) & 0.8472(3) & 0.031(2) \\ \text { H35 } & 0.507092 & 0.667211 & 0.859997 & 0.038^{*}\end{array}$

Atomic displacement parameters $\left(\AA^{2}\right)$

\begin{tabular}{lllllll}
\hline & $U^{11}$ & $U^{22}$ & $U^{33}$ & $U^{12}$ & $U^{13}$ & $U^{23}$ \\
\hline Fe1 & $0.0204(6)$ & $0.0320(7)$ & $0.0257(6)$ & $0.0000(7)$ & $0.0010(6)$ & $0.0092(5)$ \\
Fe2 & $0.0249(6)$ & $0.0264(7)$ & $0.0372(7)$ & $-0.0019(6)$ & $-0.0037(6)$ & $0.0093(6)$ \\
S1 & $0.0204(10)$ & $0.0325(12)$ & $0.0318(11)$ & $0.0038(12)$ & $-0.0019(12)$ & $0.0021(9)$ \\
P1 & $0.0197(12)$ & $0.0214(13)$ & $0.0192(11)$ & $0.0011(10)$ & $-0.0014(9)$ & $0.0017(10)$ \\
O1 & $0.022(3)$ & $0.041(4)$ & $0.044(3)$ & $-0.008(3)$ & $-0.007(3)$ & $-0.009(3)$ \\
O2 & $0.031(4)$ & $0.074(6)$ & $0.123(6)$ & $-0.012(4)$ & $0.006(4)$ & $-0.060(5)$ \\
O3 & $0.099(7)$ & $0.046(5)$ & $0.047(4)$ & $0.004(4)$ & $0.022(4)$ & $-0.014(4)$ \\
C1 & $0.014(4)$ & $0.020(5)$ & $0.018(4)$ & $-0.002(4)$ & $-0.007(3)$ & $0.006(3)$ \\
C2 & $0.024(5)$ & $0.031(5)$ & $0.011(4)$ & $0.001(4)$ & $0.000(4)$ & $0.002(4)$ \\
C3 & $0.017(5)$ & $0.044(5)$ & $0.025(4)$ & $0.000(5)$ & $0.010(4)$ & $-0.001(4)$ \\
C4 & $0.024(5)$ & $0.026(5)$ & $0.038(5)$ & $0.007(4)$ & $0.002(4)$ & $0.008(4)$ \\
C5 & $0.025(6)$ & $0.027(5)$ & $0.019(4)$ & $-0.001(4)$ & $0.000(4)$ & $0.004(4)$ \\
C6 & $0.050(8)$ & $0.056(8)$ & $0.119(11)$ & $0.026(6)$ & $0.041(8)$ & $0.062(8)$ \\
C7 & $0.075(10)$ & $0.095(11)$ & $0.085(10)$ & $-0.049(9)$ & $-0.045(9)$ & $0.077(9)$ \\
C8 & $0.093(10)$ & $0.060(8)$ & $0.041(7)$ & $-0.030(8)$ & $0.017(8)$ & $0.014(5)$ \\
C9 & $0.034(6)$ & $0.042(7)$ & $0.063(7)$ & $-0.004(5)$ & $0.008(6)$ & $0.016(5)$ \\
C10 & $0.078(8)$ & $0.026(6)$ & $0.046(6)$ & $-0.008(6)$ & $0.011(7)$ & $0.011(5)$
\end{tabular}


supporting information

$\begin{array}{lllllll}\text { C11 } & 0.029(5) & 0.033(6) & 0.024(5) & 0.000(4) & 0.000(4) & -0.011(4) \\ \text { C12 } & 0.026(6) & 0.066(7) & 0.050(6) & 0.008(7) & -0.001(6) & -0.022(5) \\ \text { C13 } & 0.069(9) & 0.047(7) & 0.100(8) & -0.016(6) & -0.017(7) & -0.013(6) \\ \text { C14 } & 0.049(7) & 0.048(6) & 0.039(5) & 0.000(6) & -0.027(5) & -0.002(5) \\ \text { C15 } & 0.021(5) & 0.026(5) & 0.018(4) & -0.003(4) & -0.004(4) & 0.005(4) \\ \text { C16 } & 0.035(6) & 0.030(6) & 0.023(5) & 0.003(4) & -0.002(4) & 0.001(4) \\ \text { C17 } & 0.045(6) & 0.043(6) & 0.012(4) & 0.006(5) & 0.001(4) & 0.009(4) \\ \text { C18 } & 0.027(5) & 0.029(5) & 0.026(5) & 0.003(4) & -0.005(4) & 0.008(4) \\ \text { C19 } & 0.019(4) & 0.024(5) & 0.027(4) & 0.002(4) & -0.002(3) & 0.002(4) \\ \text { C20 } & 0.030(6) & 0.045(7) & 0.104(9) & -0.002(6) & -0.016(6) & 0.030(7) \\ \text { C21 } & 0.047(7) & 0.060(8) & 0.054(6) & -0.027(6) & -0.028(6) & 0.013(6) \\ \text { C22 } & 0.052(8) & 0.040(7) & 0.090(8) & -0.010(6) & -0.031(7) & -0.010(6) \\ \text { C23 } & 0.038(6) & 0.039(6) & 0.101(9) & -0.016(6) & -0.039(8) & 0.022(6) \\ \text { C24 } & 0.035(6) & 0.048(7) & 0.086(8) & -0.011(6) & 0.004(6) & 0.034(6) \\ \text { C25 } & 0.055(8) & 0.050(6) & 0.028(5) & 0.015(5) & 0.011(5) & 0.004(5) \\ \text { C26 } & 0.059(7) & 0.066(8) & 0.056(7) & 0.014(6) & 0.008(6) & -0.002(5) \\ \text { C34 } & 0.026(5) & 0.025(5) & 0.022(4) & 0.001(4) & -0.006(4) & -0.005(4) \\ \text { C39 } & 0.030(5) & 0.028(6) & 0.040(5) & -0.002(4) & -0.002(4) & 0.002(4) \\ \text { C38 } & 0.041(6) & 0.021(5) & 0.055(6) & 0.001(5) & -0.009(5) & 0.002(4) \\ \text { C37 } & 0.052(7) & 0.030(5) & 0.053(6) & -0.005(5) & -0.005(5) & -0.015(5) \\ \text { C36 } & 0.056(6) & 0.039(6) & 0.045(5) & -0.003(5) & -0.017(5) & -0.004(5) \\ \text { C35 } & 0.038(5) & 0.020(4) & 0.036(5) & -0.007(4) & -0.007(5) & -0.002(4)\end{array}$

Geometric parameters $\left(\AA,{ }^{o}\right)$

\begin{tabular}{llll}
\hline $\mathrm{Fe} 1-\mathrm{C} 1$ & $2.048(7)$ & $\mathrm{C} 9-\mathrm{C} 10$ & $1.373(12)$ \\
$\mathrm{Fe} 1-\mathrm{C} 2$ & $2.067(7)$ & $\mathrm{C} 10-\mathrm{H} 10$ & 0.9500 \\
$\mathrm{Fe} 1-\mathrm{C} 3$ & $2.043(8)$ & $\mathrm{C} 11-\mathrm{H} 11$ & 1.0000 \\
$\mathrm{Fe} 1-\mathrm{C} 4$ & $2.043(8)$ & $\mathrm{C} 11-\mathrm{C} 14$ & $1.509(10)$ \\
$\mathrm{Fe} 1-\mathrm{C} 5$ & $2.021(7)$ & $\mathrm{C} 12-\mathrm{C} 13$ & $1.485(12)$ \\
$\mathrm{Fe} 1-\mathrm{C} 6$ & $2.046(10)$ & $\mathrm{C} 13-\mathrm{H} 13 \mathrm{~A}$ & 0.9800 \\
$\mathrm{Fe} 1-\mathrm{C} 7$ & $2.029(10)$ & $\mathrm{C} 13-\mathrm{H} 13 \mathrm{~B}$ & 0.9800 \\
$\mathrm{Fe} 1-\mathrm{C} 8$ & $2.022(9)$ & $\mathrm{C} 13-\mathrm{H} 13 \mathrm{C}$ & 0.9800 \\
$\mathrm{Fe} 1-\mathrm{C} 9$ & $2.013(9)$ & $\mathrm{C} 14-\mathrm{H} 14 \mathrm{~A}$ & 0.9800 \\
$\mathrm{Fe} 1-\mathrm{C} 10$ & $2.021(9)$ & $\mathrm{C} 14-\mathrm{H} 14 \mathrm{~B}$ & 0.9800 \\
$\mathrm{Fe} 2-\mathrm{C} 15$ & $2.016(8)$ & $\mathrm{C} 14-\mathrm{H} 14 \mathrm{C}$ & 0.9800 \\
$\mathrm{Fe} 2-\mathrm{C} 16$ & $2.028(8)$ & $\mathrm{C} 15-\mathrm{C} 16$ & $1.438(10)$ \\
$\mathrm{Fe} 2-\mathrm{C} 17$ & $2.038(8)$ & $\mathrm{C} 15-\mathrm{C} 19$ & $1.442(10)$ \\
$\mathrm{Fe} 2-\mathrm{C} 18$ & $2.041(8)$ & $\mathrm{C} 16-\mathrm{C} 17$ & $1.419(10)$ \\
$\mathrm{Fe} 2-\mathrm{C} 19$ & $2.045(7)$ & $\mathrm{C} 16-\mathrm{C} 25$ & $1.512(11)$ \\
$\mathrm{Fe} 2-\mathrm{C} 20$ & $2.039(9)$ & $\mathrm{C} 17-\mathrm{H} 17$ & 0.9500 \\
$\mathrm{Fe} 2-\mathrm{C} 21$ & $2.032(8)$ & $\mathrm{C} 17-\mathrm{C} 18$ & $1.415(10)$ \\
$\mathrm{Fe} 2-\mathrm{C} 22$ & $2.039(9)$ & $\mathrm{C} 18-\mathrm{H} 18$ & 0.9500 \\
$\mathrm{Fe} 2-\mathrm{C} 23$ & $2.022(9)$ & $\mathrm{C} 18-\mathrm{C} 19$ & $1.414(9)$ \\
$\mathrm{Fe} 2-\mathrm{C} 24$ & $2.052(9)$ & $\mathrm{C} 19-\mathrm{H} 19$ & 0.9500 \\
$\mathrm{~S} 1-\mathrm{P} 1$ & $1.958(3)$ & $\mathrm{C} 20-\mathrm{H} 20$ & 0.9500 \\
$\mathrm{P} 1-\mathrm{C} 1$ & $1.786(8)$ & $\mathrm{C} 20-\mathrm{C} 21$ & $1.389(13)$ \\
$\mathrm{P} 1-\mathrm{C} 15$ & $1.802(7)$ & $\mathrm{C} 20-\mathrm{C} 24$ & $1.408(12)$ \\
& & &
\end{tabular}




\begin{tabular}{|c|c|c|c|}
\hline $\mathrm{P} 1-\mathrm{C} 34$ & $1.820(8)$ & $\mathrm{C} 21-\mathrm{H} 21$ & 0.9500 \\
\hline $\mathrm{O} 1-\mathrm{C} 11$ & $1.465(9)$ & $\mathrm{C} 21-\mathrm{C} 22$ & $1.413(12)$ \\
\hline $\mathrm{O} 1-\mathrm{C} 12$ & $1.348(11)$ & $\mathrm{C} 22-\mathrm{H} 22$ & 0.9500 \\
\hline $\mathrm{O} 2-\mathrm{C} 12$ & $1.190(11)$ & $\mathrm{C} 22-\mathrm{C} 23$ & $1.399(13)$ \\
\hline $\mathrm{O} 3-\mathrm{H} 3$ & 0.8400 & $\mathrm{C} 23-\mathrm{H} 23$ & 0.9500 \\
\hline $\mathrm{O} 3-\mathrm{C} 25$ & $1.453(10)$ & $\mathrm{C} 23-\mathrm{C} 24$ & $1.404(13)$ \\
\hline $\mathrm{C} 1-\mathrm{C} 2$ & $1.456(10)$ & $\mathrm{C} 24-\mathrm{H} 24$ & 0.9500 \\
\hline $\mathrm{C} 1-\mathrm{C} 5$ & $1.433(10)$ & $\mathrm{C} 25-\mathrm{H} 25$ & 1.0000 \\
\hline $\mathrm{C} 2-\mathrm{C} 3$ & $1.417(10)$ & $\mathrm{C} 25-\mathrm{C} 26$ & $1.506(11)$ \\
\hline $\mathrm{C} 2-\mathrm{C} 11$ & $1.495(10)$ & $\mathrm{C} 26-\mathrm{H} 26 \mathrm{~A}$ & 0.9800 \\
\hline $\mathrm{C} 3-\mathrm{H} 3 \mathrm{~A}$ & 0.9500 & $\mathrm{C} 26-\mathrm{H} 26 \mathrm{~B}$ & 0.9800 \\
\hline $\mathrm{C} 3-\mathrm{C} 4$ & $1.416(10)$ & $\mathrm{C} 26-\mathrm{H} 26 \mathrm{C}$ & 0.9800 \\
\hline $\mathrm{C} 4-\mathrm{H} 4$ & 0.9500 & C34-C39 & $1.389(10)$ \\
\hline $\mathrm{C} 4-\mathrm{C} 5$ & $1.422(10)$ & $\mathrm{C} 34-\mathrm{C} 35$ & $1.391(10)$ \\
\hline C5-H5 & 0.9500 & C39-H39 & 0.9500 \\
\hline $\mathrm{C} 6-\mathrm{H} 6$ & 0.9500 & C39-C38 & $1.376(11)$ \\
\hline $\mathrm{C} 6-\mathrm{C} 7$ & $1.402(15)$ & C $38-\mathrm{H} 38$ & 0.9500 \\
\hline $\mathrm{C} 6-\mathrm{C} 10$ & $1.392(14)$ & $\mathrm{C} 38-\mathrm{C} 37$ & $1.374(11)$ \\
\hline $\mathrm{C} 7-\mathrm{H} 7$ & 0.9500 & $\mathrm{C} 37-\mathrm{H} 37$ & 0.9500 \\
\hline $\mathrm{C} 7-\mathrm{C} 8$ & $1.378(16)$ & $\mathrm{C} 37-\mathrm{C} 36$ & $1.400(11)$ \\
\hline $\mathrm{C} 8-\mathrm{H} 8$ & 0.9500 & C36- $\mathrm{H} 36$ & 0.9500 \\
\hline $\mathrm{C} 8-\mathrm{C} 9$ & $1.353(13)$ & $\mathrm{C} 36-\mathrm{C} 35$ & $1.389(10)$ \\
\hline C9-H9 & 0.9500 & $\mathrm{C} 35-\mathrm{H} 35$ & 0.9500 \\
\hline $\mathrm{C} 1-\mathrm{Fe} 1-\mathrm{C} 2$ & $41.4(3)$ & $\mathrm{C} 6-\mathrm{C} 7-\mathrm{Fe} 1$ & $70.5(6)$ \\
\hline $\mathrm{C} 3-\mathrm{Fe} 1-\mathrm{C} 1$ & $68.6(3)$ & $\mathrm{C} 6-\mathrm{C} 7-\mathrm{H} 7$ & 125.4 \\
\hline $\mathrm{C} 3-\mathrm{Fe} 1-\mathrm{C} 2$ & $40.3(3)$ & $\mathrm{C} 8-\mathrm{C} 7-\mathrm{Fe} 1$ & $69.8(7)$ \\
\hline $\mathrm{C} 3-\mathrm{Fe} 1-\mathrm{C} 4$ & $40.5(3)$ & $\mathrm{C} 8-\mathrm{C} 7-\mathrm{C} 6$ & $109.2(11)$ \\
\hline $\mathrm{C} 3-\mathrm{Fe} 1-\mathrm{C} 6$ & $155.6(5)$ & $\mathrm{C} 8-\mathrm{C} 7-\mathrm{H} 7$ & 125.4 \\
\hline $\mathrm{C} 4-\mathrm{Fe} 1-\mathrm{C} 1$ & $69.2(3)$ & $\mathrm{Fe} 1-\mathrm{C} 8-\mathrm{H} 8$ & 124.8 \\
\hline $\mathrm{C} 4-\mathrm{Fe} 1-\mathrm{C} 2$ & $68.8(3)$ & $\mathrm{C} 7-\mathrm{C} 8-\mathrm{Fe} 1$ & $70.4(6)$ \\
\hline $\mathrm{C} 4-\mathrm{Fe} 1-\mathrm{C} 6$ & $163.9(5)$ & $\mathrm{C} 7-\mathrm{C} 8-\mathrm{H} 8$ & 126.3 \\
\hline $\mathrm{C} 5-\mathrm{Fe} 1-\mathrm{C} 1$ & $41.2(3)$ & $\mathrm{C} 9-\mathrm{C} 8-\mathrm{Fe} 1$ & $70.1(6)$ \\
\hline $\mathrm{C} 5-\mathrm{Fe} 1-\mathrm{C} 2$ & $69.2(3)$ & $\mathrm{C} 9-\mathrm{C} 8-\mathrm{C} 7$ & $107.3(11)$ \\
\hline $\mathrm{C} 5-\mathrm{Fe} 1-\mathrm{C} 3$ & $68.4(3)$ & $\mathrm{C} 9-\mathrm{C} 8-\mathrm{H} 8$ & 126.3 \\
\hline $\mathrm{C} 5-\mathrm{Fe} 1-\mathrm{C} 4$ & $41.0(3)$ & $\mathrm{Fe} 1-\mathrm{C} 9-\mathrm{H} 9$ & 125.2 \\
\hline $\mathrm{C} 5-\mathrm{Fe} 1-\mathrm{C} 6$ & $130.0(5)$ & $\mathrm{C} 8-\mathrm{C} 9-\mathrm{Fe} 1$ & $70.8(6)$ \\
\hline $\mathrm{C} 5-\mathrm{Fe} 1-\mathrm{C} 7$ & $170.1(6)$ & $\mathrm{C} 8-\mathrm{C} 9-\mathrm{H} 9$ & 125.2 \\
\hline $\mathrm{C} 5-\mathrm{Fe} 1-\mathrm{C} 8$ & $145.9(5)$ & $\mathrm{C} 8-\mathrm{C} 9-\mathrm{C} 10$ & $109.6(10)$ \\
\hline $\mathrm{C} 5-\mathrm{Fe} 1-\mathrm{C} 10$ & $106.7(4)$ & $\mathrm{C} 10-\mathrm{C} 9-\mathrm{Fe} 1$ & $70.4(6)$ \\
\hline $\mathrm{C} 6-\mathrm{Fe} 1-\mathrm{C} 1$ & $112.9(4)$ & $\mathrm{C} 10-\mathrm{C} 9-\mathrm{H} 9$ & 125.2 \\
\hline $\mathrm{C} 6-\mathrm{Fe} 1-\mathrm{C} 2$ & $124.1(4)$ & $\mathrm{Fe} 1-\mathrm{C} 10-\mathrm{H} 10$ & 125.1 \\
\hline $\mathrm{C} 7-\mathrm{Fe} 1-\mathrm{C} 1$ & $134.7(5)$ & $\mathrm{C} 6-\mathrm{C} 10-\mathrm{Fe} 1$ & $70.9(6)$ \\
\hline $\mathrm{C} 7-\mathrm{Fe} 1-\mathrm{C} 2$ & $113.8(4)$ & $\mathrm{C} 6-\mathrm{C} 10-\mathrm{H} 10$ & 125.8 \\
\hline $\mathrm{C} 7-\mathrm{Fe} 1-\mathrm{C} 3$ & $120.3(5)$ & $\mathrm{C} 9-\mathrm{C} 10-\mathrm{Fe} 1$ & $69.8(5)$ \\
\hline $\mathrm{C} 7-\mathrm{Fe} 1-\mathrm{C} 4$ & $148.7(6)$ & $\mathrm{C} 9-\mathrm{C} 10-\mathrm{C} 6$ & $108.4(10)$ \\
\hline $\mathrm{C} 7-\mathrm{Fe} 1-\mathrm{C} 6$ & $40.2(4)$ & $\mathrm{C} 9-\mathrm{C} 10-\mathrm{H} 10$ & 125.8 \\
\hline $\mathrm{C} 8-\mathrm{Fe} 1-\mathrm{C} 1$ & $170.6(5)$ & $\mathrm{O} 1-\mathrm{C} 11-\mathrm{C} 2$ & $104.4(6)$ \\
\hline
\end{tabular}




$$
\begin{aligned}
& \text { C8-Fe1-C2 } \\
& \text { C8-Fe1-C3 } \\
& \text { C8-Fe1-C4 } \\
& \text { C8-Fe1-C6 } \\
& \text { C8-Fe1-C7 } \\
& \text { C9-Fe1-C1 } \\
& \text { C9-Fe1-C2 } \\
& \text { C9-Fe1-C3 } \\
& \text { C9-Fe1-C4 } \\
& \text { C9-Fe1-C5 } \\
& \text { C9-Fe1-C6 } \\
& \text { C9-Fe1-C7 } \\
& \text { C9-Fe1-C8 } \\
& \text { C9-Fe1-C10 } \\
& \text { C10-Fe1-C1 } \\
& \text { C10-Fe1-C2 } \\
& \text { C10-Fe1-C3 } \\
& \text { C10-Fe1-C4 } \\
& \text { C10-Fe1-C6 } \\
& \text { C10-Fe1-C7 } \\
& \text { C10-Fe1-C8 } \\
& \text { C15-Fe2-C16 } \\
& \text { C15-Fe2-C17 } \\
& \text { C15-Fe2-C18 } \\
& \text { C15-Fe2-C19 } \\
& \text { C15-Fe2-C20 } \\
& \text { C15-Fe2-C21 } \\
& \text { C15-Fe2-C22 } \\
& \text { C15-Fe2-C23 } \\
& \text { C15-Fe2-C24 } \\
& \text { C16-Fe2-C17 } \\
& \text { C16-Fe2-C18 } \\
& \text { C16-Fe2-C19 } \\
& \text { C16-Fe2-C20 } \\
& \text { C16-Fe2-C21 } \\
& \text { C16-Fe2-C22 } \\
& \text { C16-Fe2-C24 } \\
& \text { C17-Fe2-C18 } \\
& \text { C17-Fe2-C19 } \\
& \text { C17-Fe2-C20 } \\
& \text { C17-Fe2-C22 } \\
& \text { C17-Fe2-C24 } \\
& \text { C18-Fe2-C19 } \\
& \text { C18-Fe2-C24 } \\
& \text { C19-Fe2-C24 } \\
& \text { C20-Fe2-C18 } \\
& \text { C20-Fe2-C19 } \\
& \text { C20-Fe2-C24 }
\end{aligned}
$$

$129.9(4)$

\begin{tabular}{|c|c|}
\hline $\mathrm{O} 1-\mathrm{C} 11-\mathrm{H} 11$ & 108.9 \\
\hline $\mathrm{O} 1-\mathrm{C} 11-\mathrm{C} 14$ & $110.1(6)$ \\
\hline $\mathrm{C} 2-\mathrm{C} 11-\mathrm{H} 11$ & 108.9 \\
\hline $\mathrm{C} 2-\mathrm{C} 11-\mathrm{C} 14$ & $115.4(7)$ \\
\hline $\mathrm{C} 14-\mathrm{C} 11-\mathrm{H} 11$ & 108.9 \\
\hline $\mathrm{O} 1-\mathrm{C} 12-\mathrm{C} 13$ & $112.0(9)$ \\
\hline $\mathrm{O} 2-\mathrm{C} 12-\mathrm{O} 1$ & $123.2(8)$ \\
\hline $\mathrm{O} 2-\mathrm{C} 12-\mathrm{C} 13$ & $124.8(10)$ \\
\hline $\mathrm{C} 12-\mathrm{C} 13-\mathrm{H} 13 \mathrm{~A}$ & 109.5 \\
\hline $\mathrm{C} 12-\mathrm{C} 13-\mathrm{H} 13 \mathrm{~B}$ & 109.5 \\
\hline $\mathrm{C} 12-\mathrm{C} 13-\mathrm{H} 13 \mathrm{C}$ & 109.5 \\
\hline $\mathrm{H} 13 \mathrm{~A}-\mathrm{C} 13-\mathrm{H} 13 \mathrm{~B}$ & 109.5 \\
\hline $\mathrm{H} 13 \mathrm{~A}-\mathrm{C} 13-\mathrm{H} 13 \mathrm{C}$ & 109.5 \\
\hline $\mathrm{H} 13 \mathrm{~B}-\mathrm{C} 13-\mathrm{H} 13 \mathrm{C}$ & 109.5 \\
\hline $\mathrm{C} 11-\mathrm{C} 14-\mathrm{H} 14 \mathrm{~A}$ & 109.5 \\
\hline $\mathrm{C} 11-\mathrm{C} 14-\mathrm{H} 14 \mathrm{~B}$ & 109.5 \\
\hline $\mathrm{C} 11-\mathrm{C} 14-\mathrm{H} 14 \mathrm{C}$ & 109.5 \\
\hline $\mathrm{H} 14 \mathrm{~A}-\mathrm{C} 14-\mathrm{H} 14 \mathrm{~B}$ & 109.5 \\
\hline $\mathrm{H} 14 \mathrm{~A}-\mathrm{C} 14-\mathrm{H} 14 \mathrm{C}$ & 109.5 \\
\hline $\mathrm{H} 14 \mathrm{~B}-\mathrm{C} 14-\mathrm{H} 14 \mathrm{C}$ & 109.5 \\
\hline $\mathrm{P} 1-\mathrm{C} 15-\mathrm{Fe} 2$ & $132.2(4)$ \\
\hline $\mathrm{C} 16-\mathrm{C} 15-\mathrm{Fe} 2$ & $69.6(4)$ \\
\hline $\mathrm{C} 16-\mathrm{C} 15-\mathrm{P} 1$ & $126.2(6)$ \\
\hline $\mathrm{C} 16-\mathrm{C} 15-\mathrm{C} 19$ & $107.9(6)$ \\
\hline $\mathrm{C} 19-\mathrm{C} 15-\mathrm{Fe} 2$ & $70.3(4)$ \\
\hline $\mathrm{C} 19-\mathrm{C} 15-\mathrm{P} 1$ & $125.3(6)$ \\
\hline $\mathrm{C} 15-\mathrm{C} 16-\mathrm{Fe} 2$ & $68.8(4)$ \\
\hline $\mathrm{C} 15-\mathrm{C} 16-\mathrm{C} 25$ & $126.2(7)$ \\
\hline $\mathrm{C} 17-\mathrm{C} 16-\mathrm{Fe} 2$ & $70.0(5)$ \\
\hline $\mathrm{C} 17-\mathrm{C} 16-\mathrm{C} 15$ & $107.0(7)$ \\
\hline $\mathrm{C} 17-\mathrm{C} 16-\mathrm{C} 25$ & $126.3(7)$ \\
\hline $\mathrm{C} 25-\mathrm{C} 16-\mathrm{Fe} 2$ & $132.2(6)$ \\
\hline $\mathrm{Fe} 2-\mathrm{C} 17-\mathrm{H} 17$ & 127.2 \\
\hline $\mathrm{C} 16-\mathrm{C} 17-\mathrm{Fe} 2$ & $69.2(4)$ \\
\hline $\mathrm{C} 16-\mathrm{C} 17-\mathrm{H} 17$ & 125.5 \\
\hline $\mathrm{C} 18-\mathrm{C} 17-\mathrm{Fe} 2$ & $69.8(4)$ \\
\hline $\mathrm{C} 18-\mathrm{C} 17-\mathrm{C} 16$ & $109.1(7)$ \\
\hline $\mathrm{C} 18-\mathrm{C} 17-\mathrm{H} 17$ & 125.5 \\
\hline $\mathrm{Fe} 2-\mathrm{C} 18-\mathrm{H} 18$ & 126.3 \\
\hline $\mathrm{C} 17-\mathrm{C} 18-\mathrm{Fe} 2$ & $69.6(5)$ \\
\hline $\mathrm{C} 17-\mathrm{C} 18-\mathrm{H} 18$ & 125.7 \\
\hline $\mathrm{C} 19-\mathrm{C} 18-\mathrm{Fe} 2$ & $69.9(4)$ \\
\hline $\mathrm{C} 19-\mathrm{C} 18-\mathrm{C} 17$ & $108.5(7)$ \\
\hline $\mathrm{C} 19-\mathrm{C} 18-\mathrm{H} 18$ & 125.7 \\
\hline $\mathrm{Fe} 2-\mathrm{C} 19-\mathrm{H} 19$ & 127.5 \\
\hline $\mathrm{C} 15-\mathrm{C} 19-\mathrm{Fe} 2$ & $68.1(4)$ \\
\hline $\mathrm{C} 15-\mathrm{C} 19-\mathrm{H} 19$ & 126.3 \\
\hline $\mathrm{C} 18-\mathrm{C} 19-\mathrm{Fe} 2$ & $69.6(4)$ \\
\hline
\end{tabular}

106.7 (4)

$113.0(5)$

$67.7(5)$

$39.8(4)$

$150.2(4)$

$163.8(4)$

$124.5(4)$

$102.8(4)$

$114.1(4)$

$67.1(4)$

$65.9(4)$

$39.2(4)$

39.8 (4)

$120.1(4)$

$156.3(4)$

161.7 (4)

$124.3(4)$

40.0 (4)

$66.6(4)$

$66.9(4)$

$41.6(3)$

$69.0(3)$

$69.1(3)$

$41.6(3)$

$119.8(4)$

$109.9(3)$

$129.4(4)$

166.7 (4)

$152.2(4)$

40.8 (3)

$69.1(3)$

69.7 (3)

$109.7(4)$

130.6 (4)

169.0 (4)

117.4 (4)

40.6 (3)

68.4 (3)

129.6 (4)

149.1 (4)

107.4 (4)

40.5 (3)

127.0 (3)

164.7 (4)

166.1 (4)

153.2 (3)

$40.3(3)$ 


\begin{tabular}{|c|c|}
\hline $\mathrm{C} 21-\mathrm{Fe} 2-\mathrm{C} 17$ & $168.2(4)$ \\
\hline $\mathrm{C} 21-\mathrm{Fe} 2-\mathrm{C} 18$ & $151.0(4)$ \\
\hline $\mathrm{C} 21-\mathrm{Fe} 2-\mathrm{C} 19$ & $119.1(4)$ \\
\hline $\mathrm{C} 21-\mathrm{Fe} 2-\mathrm{C} 20$ & 39.9 (4) \\
\hline $\mathrm{C} 21-\mathrm{Fe} 2-\mathrm{C} 22$ & $40.6(4)$ \\
\hline $\mathrm{C} 21-\mathrm{Fe} 2-\mathrm{C} 24$ & $67.7(4)$ \\
\hline $\mathrm{C} 22-\mathrm{Fe} 2-\mathrm{C} 18$ & $116.2(4)$ \\
\hline $\mathrm{C} 22-\mathrm{Fe} 2-\mathrm{C} 19$ & $107.5(4)$ \\
\hline $\mathrm{C} 22-\mathrm{Fe} 2-\mathrm{C} 20$ & $67.7(5)$ \\
\hline $\mathrm{C} 22-\mathrm{Fe} 2-\mathrm{C} 24$ & $68.0(4)$ \\
\hline $\mathrm{C} 23-\mathrm{Fe} 2-\mathrm{C} 16$ & $149.8(4)$ \\
\hline $\mathrm{C} 23-\mathrm{Fe} 2-\mathrm{C} 17$ & $116.2(4)$ \\
\hline $\mathrm{C} 23-\mathrm{Fe} 2-\mathrm{C} 18$ & $106.3(4)$ \\
\hline $\mathrm{C} 23-\mathrm{Fe} 2-\mathrm{C} 19$ & $127.1(4)$ \\
\hline $\mathrm{C} 23-\mathrm{Fe} 2-\mathrm{C} 20$ & $67.4(4)$ \\
\hline $\mathrm{C} 23-\mathrm{Fe} 2-\mathrm{C} 21$ & $67.7(4)$ \\
\hline $\mathrm{C} 23-\mathrm{Fe} 2-\mathrm{C} 22$ & $40.3(4)$ \\
\hline $\mathrm{C} 23-\mathrm{Fe} 2-\mathrm{C} 24$ & $40.3(4)$ \\
\hline $\mathrm{C} 1-\mathrm{P} 1-\mathrm{S} 1$ & $116.0(2)$ \\
\hline $\mathrm{C} 1-\mathrm{P} 1-\mathrm{C} 15$ & $103.4(3)$ \\
\hline $\mathrm{C} 1-\mathrm{P} 1-\mathrm{C} 34$ & $104.3(4)$ \\
\hline $\mathrm{C} 15-\mathrm{P} 1-\mathrm{S} 1$ & $116.5(3)$ \\
\hline $\mathrm{C} 15-\mathrm{P} 1-\mathrm{C} 34$ & $102.4(3)$ \\
\hline $\mathrm{C} 34-\mathrm{P} 1-\mathrm{S} 1$ & $112.7(3)$ \\
\hline $\mathrm{C} 12-\mathrm{O} 1-\mathrm{C} 11$ & $118.1(7)$ \\
\hline $\mathrm{C} 25-\mathrm{O} 3-\mathrm{H} 3$ & 109.5 \\
\hline $\mathrm{P} 1-\mathrm{C} 1-\mathrm{Fe} 1$ & $130.2(4)$ \\
\hline $\mathrm{C} 2-\mathrm{C} 1-\mathrm{Fe} 1$ & $70.0(4)$ \\
\hline $\mathrm{C} 2-\mathrm{C} 1-\mathrm{P} 1$ & $130.2(6)$ \\
\hline $\mathrm{C} 5-\mathrm{C} 1-\mathrm{Fe} 1$ & $68.4(4)$ \\
\hline $\mathrm{C} 5-\mathrm{C} 1-\mathrm{P} 1$ & $122.6(6)$ \\
\hline $\mathrm{C} 5-\mathrm{C} 1-\mathrm{C} 2$ & $107.0(7)$ \\
\hline $\mathrm{C} 1-\mathrm{C} 2-\mathrm{Fe} 1$ & $68.6(4)$ \\
\hline $\mathrm{C} 1-\mathrm{C} 2-\mathrm{C} 11$ & $129.1(7)$ \\
\hline $\mathrm{C} 3-\mathrm{C} 2-\mathrm{Fe} 1$ & $68.9(4)$ \\
\hline $\mathrm{C} 3-\mathrm{C} 2-\mathrm{C} 1$ & $106.8(6)$ \\
\hline $\mathrm{C} 3-\mathrm{C} 2-\mathrm{C} 11$ & $123.9(7)$ \\
\hline $\mathrm{C} 11-\mathrm{C} 2-\mathrm{Fe} 1$ & $131.9(5)$ \\
\hline $\mathrm{Fe} 1-\mathrm{C} 3-\mathrm{H} 3 \mathrm{~A}$ & 126.1 \\
\hline $\mathrm{C} 2-\mathrm{C} 3-\mathrm{Fe} 1$ & $70.8(4)$ \\
\hline $\mathrm{C} 2-\mathrm{C} 3-\mathrm{H} 3 \mathrm{~A}$ & 125.0 \\
\hline $\mathrm{C} 4-\mathrm{C} 3-\mathrm{Fe} 1$ & $69.7(4)$ \\
\hline $\mathrm{C} 4-\mathrm{C} 3-\mathrm{C} 2$ & $110.1(7)$ \\
\hline $\mathrm{C} 4-\mathrm{C} 3-\mathrm{H} 3 \mathrm{~A}$ & 125.0 \\
\hline $\mathrm{Fe} 1-\mathrm{C} 4-\mathrm{H} 4$ & 126.7 \\
\hline $\mathrm{C} 3-\mathrm{C} 4-\mathrm{Fe} 1$ & $69.7(4)$ \\
\hline $\mathrm{C} 3-\mathrm{C} 4-\mathrm{H} 4$ & 126.4 \\
\hline $\mathrm{C} 3-\mathrm{C} 4-\mathrm{C} 5$ & $107.2(7)$ \\
\hline
\end{tabular}

\begin{tabular}{|c|c|}
\hline $\mathrm{C} 18-\mathrm{C} 19-\mathrm{C} 15$ & $107.4(7)$ \\
\hline $\mathrm{C} 18-\mathrm{C} 19-\mathrm{H} 19$ & 126.3 \\
\hline $\mathrm{Fe} 2-\mathrm{C} 20-\mathrm{H} 20$ & 125.9 \\
\hline $\mathrm{C} 21-\mathrm{C} 20-\mathrm{Fe} 2$ & $69.8(5)$ \\
\hline $\mathrm{C} 21-\mathrm{C} 20-\mathrm{H} 20$ & 125.5 \\
\hline $\mathrm{C} 21-\mathrm{C} 20-\mathrm{C} 24$ & $108.9(9)$ \\
\hline $\mathrm{C} 24-\mathrm{C} 20-\mathrm{Fe} 2$ & $70.3(5)$ \\
\hline $\mathrm{C} 24-\mathrm{C} 20-\mathrm{H} 20$ & 125.5 \\
\hline $\mathrm{Fe} 2-\mathrm{C} 21-\mathrm{H} 21$ & 125.4 \\
\hline $\mathrm{C} 20-\mathrm{C} 21-\mathrm{Fe} 2$ & $70.3(5)$ \\
\hline $\mathrm{C} 20-\mathrm{C} 21-\mathrm{H} 21$ & 125.9 \\
\hline $\mathrm{C} 20-\mathrm{C} 21-\mathrm{C} 22$ & $108.3(9)$ \\
\hline $\mathrm{C} 22-\mathrm{C} 21-\mathrm{Fe} 2$ & $69.9(5)$ \\
\hline $\mathrm{C} 22-\mathrm{C} 21-\mathrm{H} 21$ & 125.9 \\
\hline $\mathrm{Fe} 2-\mathrm{C} 22-\mathrm{H} 22$ & 126.3 \\
\hline $\mathrm{C} 21-\mathrm{C} 22-\mathrm{Fe} 2$ & $69.4(5)$ \\
\hline $\mathrm{C} 21-\mathrm{C} 22-\mathrm{H} 22$ & 126.6 \\
\hline $\mathrm{C} 23-\mathrm{C} 22-\mathrm{Fe} 2$ & $69.2(5)$ \\
\hline $\mathrm{C} 23-\mathrm{C} 22-\mathrm{C} 21$ & $106.8(10$ \\
\hline $\mathrm{C} 23-\mathrm{C} 22-\mathrm{H} 22$ & 126.6 \\
\hline $\mathrm{Fe} 2-\mathrm{C} 23-\mathrm{H} 23$ & 124.9 \\
\hline $\mathrm{C} 22-\mathrm{C} 23-\mathrm{Fe} 2$ & $70.5(5)$ \\
\hline $\mathrm{C} 22-\mathrm{C} 23-\mathrm{H} 23$ & 125.3 \\
\hline $\mathrm{C} 22-\mathrm{C} 23-\mathrm{C} 24$ & $109.5(9)$ \\
\hline $\mathrm{C} 24-\mathrm{C} 23-\mathrm{Fe} 2$ & $71.0(5)$ \\
\hline $\mathrm{C} 24-\mathrm{C} 23-\mathrm{H} 23$ & 125.3 \\
\hline $\mathrm{Fe} 2-\mathrm{C} 24-\mathrm{H} 24$ & 126.7 \\
\hline $\mathrm{C} 20-\mathrm{C} 24-\mathrm{Fe} 2$ & $69.4(5)$ \\
\hline $\mathrm{C} 20-\mathrm{C} 24-\mathrm{H} 24$ & 126.8 \\
\hline $\mathrm{C} 23-\mathrm{C} 24-\mathrm{Fe} 2$ & $68.7(5)$ \\
\hline $\mathrm{C} 23-\mathrm{C} 24-\mathrm{C} 20$ & $106.5(10$ \\
\hline $\mathrm{C} 23-\mathrm{C} 24-\mathrm{H} 24$ & 126.8 \\
\hline $\mathrm{O} 3-\mathrm{C} 25-\mathrm{C} 16$ & $109.2(7)$ \\
\hline $\mathrm{O} 3-\mathrm{C} 25-\mathrm{H} 25$ & 109.1 \\
\hline $\mathrm{O} 3-\mathrm{C} 25-\mathrm{C} 26$ & $106.3(7)$ \\
\hline $\mathrm{C} 16-\mathrm{C} 25-\mathrm{H} 25$ & 109.1 \\
\hline $\mathrm{C} 26-\mathrm{C} 25-\mathrm{C} 16$ & $114.1(8)$ \\
\hline $\mathrm{C} 26-\mathrm{C} 25-\mathrm{H} 25$ & 109.1 \\
\hline $\mathrm{C} 25-\mathrm{C} 26-\mathrm{H} 26 \mathrm{~A}$ & 109.5 \\
\hline $\mathrm{C} 25-\mathrm{C} 26-\mathrm{H} 26 \mathrm{~B}$ & 109.5 \\
\hline $\mathrm{C} 25-\mathrm{C} 26-\mathrm{H} 26 \mathrm{C}$ & 109.5 \\
\hline $\mathrm{H} 26 \mathrm{~A}-\mathrm{C} 26-\mathrm{H} 26 \mathrm{~B}$ & 109.5 \\
\hline $\mathrm{H} 26 \mathrm{~A}-\mathrm{C} 26-\mathrm{H} 26 \mathrm{C}$ & 109.5 \\
\hline $\mathrm{H} 26 \mathrm{~B}-\mathrm{C} 26-\mathrm{H} 26 \mathrm{C}$ & 109.5 \\
\hline $\mathrm{C} 39-\mathrm{C} 34-\mathrm{P} 1$ & $120.0(6)$ \\
\hline $\mathrm{C} 39-\mathrm{C} 34-\mathrm{C} 35$ & $119.0(7)$ \\
\hline $\mathrm{C} 35-\mathrm{C} 34-\mathrm{P} 1$ & $121.0(6)$ \\
\hline $\mathrm{C} 34-\mathrm{C} 39-\mathrm{H} 39$ & 119.5 \\
\hline
\end{tabular}




\begin{tabular}{|c|c|c|c|}
\hline $\mathrm{C} 5-\mathrm{C} 4-\mathrm{Fe} 1$ & $68.7(4)$ & $\mathrm{C} 38-\mathrm{C} 39-\mathrm{C} 34$ & $120.9(8)$ \\
\hline $\mathrm{C} 5-\mathrm{C} 4-\mathrm{H} 4$ & 126.4 & $\mathrm{C} 38-\mathrm{C} 39-\mathrm{H} 39$ & 119.5 \\
\hline $\mathrm{Fe} 1-\mathrm{C} 5-\mathrm{H} 5$ & 125.3 & $\mathrm{C} 39-\mathrm{C} 38-\mathrm{H} 38$ & 120.0 \\
\hline $\mathrm{C} 1-\mathrm{C} 5-\mathrm{Fe} 1$ & $70.4(4)$ & $\mathrm{C} 37-\mathrm{C} 38-\mathrm{C} 39$ & $120.0(8)$ \\
\hline $\mathrm{C} 1-\mathrm{C} 5-\mathrm{H} 5$ & 125.5 & $\mathrm{C} 37-\mathrm{C} 38-\mathrm{H} 38$ & 120.0 \\
\hline $\mathrm{C} 4-\mathrm{C} 5-\mathrm{Fe} 1$ & $70.3(4)$ & $\mathrm{C} 38-\mathrm{C} 37-\mathrm{H} 37$ & 119.8 \\
\hline $\mathrm{C} 4-\mathrm{C} 5-\mathrm{C} 1$ & $109.0(7)$ & $\mathrm{C} 38-\mathrm{C} 37-\mathrm{C} 36$ & $120.4(8)$ \\
\hline $\mathrm{C} 4-\mathrm{C} 5-\mathrm{H} 5$ & 125.5 & $\mathrm{C} 36-\mathrm{C} 37-\mathrm{H} 37$ & 119.8 \\
\hline $\mathrm{Fe} 1-\mathrm{C} 6-\mathrm{H} 6$ & 126.0 & $\mathrm{C} 37-\mathrm{C} 36-\mathrm{H} 36$ & 120.5 \\
\hline $\mathrm{C} 7-\mathrm{C} 6-\mathrm{Fe} 1$ & $69.3(7)$ & $\mathrm{C} 35-\mathrm{C} 36-\mathrm{C} 37$ & $119.1(8)$ \\
\hline $\mathrm{C} 7-\mathrm{C} 6-\mathrm{H} 6$ & 127.3 & $\mathrm{C} 35-\mathrm{C} 36-\mathrm{H} 36$ & 120.5 \\
\hline $\mathrm{C} 10-\mathrm{C} 6-\mathrm{Fe} 1$ & $69.0(6)$ & $\mathrm{C} 34-\mathrm{C} 35-\mathrm{H} 35$ & 119.7 \\
\hline $\mathrm{C} 10-\mathrm{C} 6-\mathrm{H} 6$ & 127.3 & $\mathrm{C} 36-\mathrm{C} 35-\mathrm{C} 34$ & $120.6(8)$ \\
\hline $\mathrm{C} 10-\mathrm{C} 6-\mathrm{C} 7$ & $105.5(11)$ & $\mathrm{C} 36-\mathrm{C} 35-\mathrm{H} 35$ & 119.7 \\
\hline $\mathrm{Fe} 1-\mathrm{C} 7-\mathrm{H} 7$ & 125.9 & & \\
\hline $\mathrm{Fe} 1-\mathrm{C} 1-\mathrm{C} 2-\mathrm{C} 3$ & $58.5(5)$ & $\mathrm{C} 5-\mathrm{C} 1-\mathrm{C} 2-\mathrm{Fe} 1$ & $-58.6(5)$ \\
\hline $\mathrm{Fe} 1-\mathrm{C} 1-\mathrm{C} 2-\mathrm{C} 11$ & $-127.3(8)$ & $\mathrm{C} 5-\mathrm{C} 1-\mathrm{C} 2-\mathrm{C} 3$ & $-0.1(8)$ \\
\hline $\mathrm{Fe} 1-\mathrm{C} 1-\mathrm{C} 5-\mathrm{C} 4$ & $-60.0(5)$ & $\mathrm{C} 5-\mathrm{C} 1-\mathrm{C} 2-\mathrm{C} 11$ & $174.1(7)$ \\
\hline $\mathrm{Fe} 1-\mathrm{C} 2-\mathrm{C} 3-\mathrm{C} 4$ & $58.8(5)$ & $\mathrm{C} 6-\mathrm{C} 7-\mathrm{C} 8-\mathrm{Fe} 1$ & $59.7(7)$ \\
\hline $\mathrm{Fe} 1-\mathrm{C} 2-\mathrm{C} 11-\mathrm{O} 1$ & $135.7(6)$ & $\mathrm{C} 6-\mathrm{C} 7-\mathrm{C} 8-\mathrm{C} 9$ & $-1.0(13)$ \\
\hline $\mathrm{Fe} 1-\mathrm{C} 2-\mathrm{C} 11-\mathrm{C} 14$ & $14.7(11)$ & $\mathrm{C} 7-\mathrm{C} 6-\mathrm{C} 10-\mathrm{Fe} 1$ & $-60.0(7)$ \\
\hline $\mathrm{Fe} 1-\mathrm{C} 3-\mathrm{C} 4-\mathrm{C} 5$ & $58.7(5)$ & $\mathrm{C} 7-\mathrm{C} 6-\mathrm{C} 10-\mathrm{C} 9$ & $-0.1(11)$ \\
\hline $\mathrm{Fe} 1-\mathrm{C} 4-\mathrm{C} 5-\mathrm{C} 1$ & $60.0(5)$ & $\mathrm{C} 7-\mathrm{C} 8-\mathrm{C} 9-\mathrm{Fe} 1$ & $60.9(8)$ \\
\hline $\mathrm{Fe} 1-\mathrm{C} 6-\mathrm{C} 7-\mathrm{C} 8$ & $-59.3(8)$ & $\mathrm{C} 7-\mathrm{C} 8-\mathrm{C} 9-\mathrm{C} 10$ & $0.9(12)$ \\
\hline $\mathrm{Fe} 1-\mathrm{C} 6-\mathrm{C} 10-\mathrm{C} 9$ & $60.0(6)$ & $\mathrm{C} 8-\mathrm{C} 9-\mathrm{C} 10-\mathrm{Fe} 1$ & $60.2(7)$ \\
\hline $\mathrm{Fe} 1-\mathrm{C} 7-\mathrm{C} 8-\mathrm{C} 9$ & $-60.6(7)$ & $\mathrm{C} 8-\mathrm{C} 9-\mathrm{C} 10-\mathrm{C} 6$ & $-0.5(11)$ \\
\hline $\mathrm{Fe} 1-\mathrm{C} 8-\mathrm{C} 9-\mathrm{C} 10$ & $-60.0(7)$ & $\mathrm{C} 10-\mathrm{C} 6-\mathrm{C} 7-\mathrm{Fe} 1$ & $59.9(7)$ \\
\hline $\mathrm{Fe} 1-\mathrm{C} 9-\mathrm{C} 10-\mathrm{C} 6$ & $-60.7(7)$ & $\mathrm{C} 10-\mathrm{C} 6-\mathrm{C} 7-\mathrm{C} 8$ & $0.6(12)$ \\
\hline $\mathrm{Fe} 2-\mathrm{C} 15-\mathrm{C} 16-\mathrm{C} 17$ & $59.8(6)$ & $\mathrm{C} 11-\mathrm{O} 1-\mathrm{C} 12-\mathrm{O} 2$ & $1.4(13)$ \\
\hline $\mathrm{Fe} 2-\mathrm{C} 15-\mathrm{C} 16-\mathrm{C} 25$ & $-127.5(8)$ & $\mathrm{C} 11-\mathrm{O} 1-\mathrm{C} 12-\mathrm{C} 13$ & $-177.3(7)$ \\
\hline $\mathrm{Fe} 2-\mathrm{C} 15-\mathrm{C} 19-\mathrm{C} 18$ & $-58.7(5)$ & $\mathrm{C} 11-\mathrm{C} 2-\mathrm{C} 3-\mathrm{Fe} 1$ & $127.1(7)$ \\
\hline $\mathrm{Fe} 2-\mathrm{C} 16-\mathrm{C} 17-\mathrm{C} 18$ & $58.5(6)$ & $\mathrm{C} 11-\mathrm{C} 2-\mathrm{C} 3-\mathrm{C} 4$ & $-174.1(7)$ \\
\hline $\mathrm{Fe} 2-\mathrm{C} 16-\mathrm{C} 25-\mathrm{O} 3$ & $172.7(6)$ & $\mathrm{C} 12-\mathrm{O} 1-\mathrm{C} 11-\mathrm{C} 2$ & $164.2(7)$ \\
\hline $\mathrm{Fe} 2-\mathrm{C} 16-\mathrm{C} 25-\mathrm{C} 26$ & $53.9(10)$ & $\mathrm{C} 12-\mathrm{O} 1-\mathrm{C} 11-\mathrm{C} 14$ & $-71.3(9)$ \\
\hline $\mathrm{Fe} 2-\mathrm{C} 17-\mathrm{C} 18-\mathrm{C} 19$ & $59.3(5)$ & $\mathrm{C} 15-\mathrm{P} 1-\mathrm{C} 1-\mathrm{Fe} 1$ & $-155.6(5)$ \\
\hline $\mathrm{Fe} 2-\mathrm{C} 18-\mathrm{C} 19-\mathrm{C} 15$ & $57.8(5)$ & $\mathrm{C} 15-\mathrm{P} 1-\mathrm{C} 1-\mathrm{C} 2$ & $106.9(7)$ \\
\hline $\mathrm{Fe} 2-\mathrm{C} 20-\mathrm{C} 21-\mathrm{C} 22$ & $59.9(6)$ & $\mathrm{C} 15-\mathrm{P} 1-\mathrm{C} 1-\mathrm{C} 5$ & $-67.5(7)$ \\
\hline $\mathrm{Fe} 2-\mathrm{C} 20-\mathrm{C} 24-\mathrm{C} 23$ & $-59.0(6)$ & $\mathrm{C} 15-\mathrm{P} 1-\mathrm{C} 34-\mathrm{C} 39$ & $-148.6(6)$ \\
\hline $\mathrm{Fe} 2-\mathrm{C} 21-\mathrm{C} 22-\mathrm{C} 23$ & $59.4(7)$ & $\mathrm{C} 15-\mathrm{P} 1-\mathrm{C} 34-\mathrm{C} 35$ & $31.8(7)$ \\
\hline $\mathrm{Fe} 2-\mathrm{C} 22-\mathrm{C} 23-\mathrm{C} 24$ & $60.5(7)$ & $\mathrm{C} 15-\mathrm{C} 16-\mathrm{C} 17-\mathrm{Fe} 2$ & $-59.0(5)$ \\
\hline $\mathrm{Fe} 2-\mathrm{C} 23-\mathrm{C} 24-\mathrm{C} 20$ & $59.4(6)$ & $\mathrm{C} 15-\mathrm{C} 16-\mathrm{C} 17-\mathrm{C} 18$ & $-0.5(9)$ \\
\hline $\mathrm{S} 1-\mathrm{P} 1-\mathrm{C} 1-\mathrm{Fe} 1$ & $75.6(5)$ & $\mathrm{C} 15-\mathrm{C} 16-\mathrm{C} 25-\mathrm{O} 3$ & $-94.0(9)$ \\
\hline $\mathrm{S} 1-\mathrm{P} 1-\mathrm{C} 1-\mathrm{C} 2$ & $-21.8(8)$ & $\mathrm{C} 15-\mathrm{C} 16-\mathrm{C} 25-\mathrm{C} 26$ & $147.3(8)$ \\
\hline $\mathrm{S} 1-\mathrm{P} 1-\mathrm{C} 1-\mathrm{C} 5$ & $163.8(5)$ & $\mathrm{C} 16-\mathrm{C} 15-\mathrm{C} 19-\mathrm{Fe} 2$ & $59.7(5)$ \\
\hline $\mathrm{S} 1-\mathrm{P} 1-\mathrm{C} 15-\mathrm{Fe} 2$ & $37.9(6)$ & $\mathrm{C} 16-\mathrm{C} 15-\mathrm{C} 19-\mathrm{C} 18$ & $1.0(8)$ \\
\hline $\mathrm{S} 1-\mathrm{P} 1-\mathrm{C} 15-\mathrm{C} 16$ & $-56.7(7)$ & $\mathrm{C} 16-\mathrm{C} 17-\mathrm{C} 18-\mathrm{Fe} 2$ & $-58.1(6)$ \\
\hline $\mathrm{S} 1-\mathrm{P} 1-\mathrm{C} 15-\mathrm{C} 19$ & $132.7(6)$ & $\mathrm{C} 16-\mathrm{C} 17-\mathrm{C} 18-\mathrm{C} 19$ & $1.1(9)$ \\
\hline
\end{tabular}




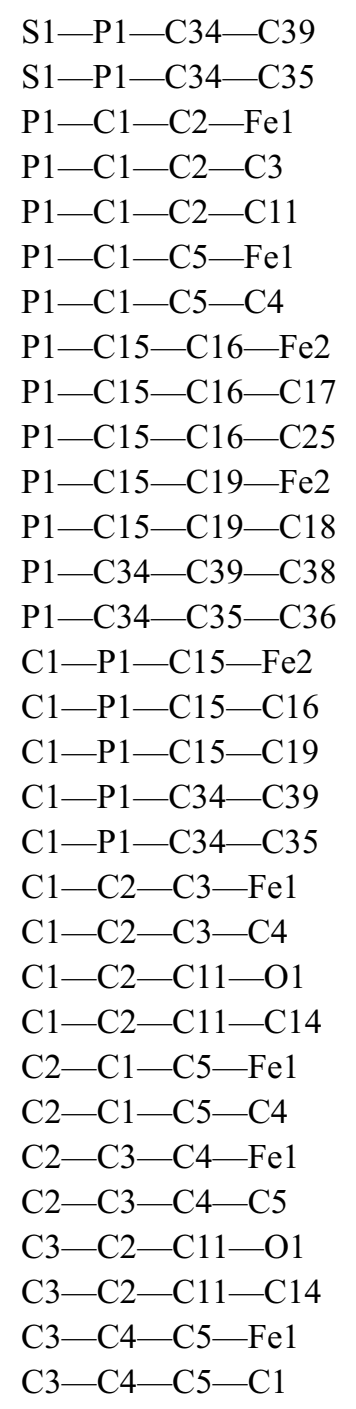

$-22.8(7)$
$157.7(5)$
$126.3(6)$
$-175.2(6)$
$-1.0(12)$
$-124.8(6)$
$175.2(5)$
$128.0(6)$
$-172.2(6)$
$0.5(12)$
$-128.3(6)$
$173.0(6)$
$-177.3(6)$
$177.3(6)$
$-90.6(6)$
$174.8(7)$
$4.2(7)$
$103.8(7)$
$-75.7(6)$
$-58.3(5)$
$0.5(8)$
$-128.7(8)$
$110.3(9)$
$59.6(5)$
$-0.4(8)$
$-59.4(6)$
$-0.7(9)$
$44.6(9)$
$-76.4(10)$
$-59.3(5)$
$0.7(9)$

$\begin{array}{ll}\mathrm{C} 17-\mathrm{C} 16-\mathrm{C} 25-\mathrm{O} 3 & 77.3(10) \\ \mathrm{C} 17-\mathrm{C} 16-\mathrm{C} 25-\mathrm{C} 26 & -41.5(12) \\ \mathrm{C} 17-\mathrm{C} 18-\mathrm{C} 19-\mathrm{Fe} 2 & -59.1(6) \\ \mathrm{C} 17-\mathrm{C} 18-\mathrm{C} 19-\mathrm{C} 15 & -1.3(8) \\ \mathrm{C} 19-\mathrm{C} 15-\mathrm{C} 16-\mathrm{Fe} 2 & -60.1(5) \\ \mathrm{C} 19-\mathrm{C} 15-\mathrm{C} 16-\mathrm{C} 17 & -0.3(9) \\ \mathrm{C} 19-\mathrm{C} 15-\mathrm{C} 16-\mathrm{C} 25 & 172.4(8) \\ \mathrm{C} 20-\mathrm{C} 21-\mathrm{C} 22-\mathrm{Fe} 2 & -60.2(7) \\ \mathrm{C} 20-\mathrm{C} 21-\mathrm{C} 22-\mathrm{C} 23 & -0.8(11) \\ \mathrm{C} 21-\mathrm{C} 20-\mathrm{C} 24-\mathrm{Fe} 2 & 59.3(7) \\ \mathrm{C} 21-\mathrm{C} 20-\mathrm{C} 24-\mathrm{C} 23 & 0.3(11) \\ \mathrm{C} 21-\mathrm{C} 22-\mathrm{C} 23-\mathrm{Fe} 2 & -59.5(6) \\ \mathrm{C} 21-\mathrm{C} 22-\mathrm{C} 23-\mathrm{C} 24 & 1.0(11) \\ \mathrm{C} 22-\mathrm{C} 23-\mathrm{C} 24-\mathrm{Fe} 2 & -60.2(7) \\ \mathrm{C} 22-\mathrm{C} 23-\mathrm{C} 24-\mathrm{C} 20 & -0.8(11) \\ \mathrm{C} 24-\mathrm{C} 20-\mathrm{C} 21-\mathrm{Fe} 2 & -59.6(7) \\ \mathrm{C} 24-\mathrm{C} 20-\mathrm{C} 21-\mathrm{C} 22 & 0.3(11) \\ \mathrm{C} 25-\mathrm{C} 16-\mathrm{C} 17-\mathrm{Fe} 2 & 128.3(9) \\ \mathrm{C} 25-\mathrm{C} 16-\mathrm{C} 17-\mathrm{C} 18 & -173.2(8) \\ \mathrm{C} 34-\mathrm{P} 1-\mathrm{C} 1-\mathrm{Fe} 1 & -48.9(6) \\ \mathrm{C} 34-\mathrm{P} 1-\mathrm{C} 1-\mathrm{C} 2 & -146.3(7) \\ \mathrm{C} 34-\mathrm{P} 1-\mathrm{C} 1-\mathrm{C} 5 & 39.3(7) \\ \mathrm{C} 34-\mathrm{P} 1-\mathrm{C} 15-\mathrm{Fe} 2 & 161.2(5) \\ \mathrm{C} 34-\mathrm{P} 1-\mathrm{C} 15-\mathrm{C} 16 & 66.6(7) \\ \mathrm{C} 34-\mathrm{P} 1-\mathrm{C} 15-\mathrm{C} 19 & -103.9(7) \\ \mathrm{C} 34-\mathrm{C} 39-\mathrm{C} 38-\mathrm{C} 37 & -0.4(13) \\ \mathrm{C} 39-\mathrm{C} 34-\mathrm{C} 35-\mathrm{C} 36 & -2.2(11) \\ \mathrm{C} 39-\mathrm{C} 38-\mathrm{C} 37-\mathrm{C} 36 & -1.5(13) \\ \mathrm{C} 38-\mathrm{C} 37-\mathrm{C} 36-\mathrm{C} 35 & 1.5(13) \\ \mathrm{C} 37-\mathrm{C} 36-\mathrm{C} 35-\mathrm{C} 34 & 0.4(12) \\ \mathrm{C} 35-\mathrm{C} 34-\mathrm{C} 39-\mathrm{C} 38 & 2.2(12) \\ & \end{array}$

$\left\{\left(2 S_{\mathrm{p}}\right)-2-[(1 R)-1-B e n z y l a m i n o)\right.$ ethyl] ferrocen-1-yl $\}\left[\left(2 S_{\mathrm{p}}\right)\right.$-2-ethenylferrocen-1-yl]phenyl-(S)-phosphane sulfide (9b)

\section{Crystal data}

$\left[\mathrm{Fe}_{2}\left(\mathrm{C}_{5} \mathrm{H}_{5}\right)_{2}\left(\mathrm{C}_{27} \mathrm{H}_{26} \mathrm{NPS}\right)\right]$

$M_{r}=669.40$

Orthorhombic, $P 2{ }_{1} 2_{1} 2_{1}$

$a=12.3578$ (9) $\AA$

$b=14.4342(10) \AA$

$c=17.4796(15) \AA$

$V=3117.9(4) \AA^{3}$

$Z=4$

$F(000)=1392$
$D_{\mathrm{x}}=1.426 \mathrm{Mg} \mathrm{m}^{-3}$

Mo $K \alpha$ radiation, $\lambda=0.71073 \AA$

Cell parameters from 9501 reflections

$\theta=2.2-26.6^{\circ}$

$\mu=1.08 \mathrm{~mm}^{-1}$

$T=130 \mathrm{~K}$

Block, clear orange $0.22 \times 0.11 \times 0.09 \mathrm{~mm}$ 


\section{Data collection}

Bruker APEXII CCD

diffractometer

Radiation source: sealed xray tube, Incoatec IuS

$\varphi$ and $\omega$ scans

Absorption correction: multi-scan

(SADABS; Bruker, 2008)

$T_{\min }=0.486, T_{\max }=0.746$

38997 measured reflections

\section{Refinement}

Refinement on $F^{2}$

Least-squares matrix: full

$R\left[F^{2}>2 \sigma\left(F^{2}\right)\right]=0.040$

$w R\left(F^{2}\right)=0.091$

$S=0.98$

9160 reflections

384 parameters

0 restraints

Primary atom site location: structure-invariant direct methods

Hydrogen site location: mixed
9160 independent reflections

7062 reflections with $I>2 \sigma(I)$

$R_{\text {int }}=0.075$

$\theta_{\max }=30.1^{\circ}, \theta_{\min }=2.2^{\circ}$

$h=-17 \rightarrow 16$

$k=-20 \rightarrow 17$

$l=-24 \rightarrow 24$
$\mathrm{H}$ atoms treated by a mixture of independent and constrained refinement

$w=1 /\left[\sigma^{2}\left(F_{\mathrm{o}}^{2}\right)+(0.0377 P)^{2}\right]$ where $P=\left(F_{\mathrm{o}}^{2}+2 F_{\mathrm{c}}^{2}\right) / 3$

$(\Delta / \sigma)_{\max }=0.001$

$\Delta \rho_{\max }=0.43$ e $\AA^{-3}$

$\Delta \rho_{\min }=-0.35$ e $\AA^{-3}$

Absolute structure: Flack $x$ determined using 2549 quotients [(I+)-(I-)]/[(I+)+(I-)] (Parsons et al., 2013)

Absolute structure parameter: $-0.016(10)$

\section{Special details}

Geometry. All esds (except the esd in the dihedral angle between two 1.s. planes) are estimated using the full covariance matrix. The cell esds are taken into account individually in the estimation of esds in distances, angles and torsion angles; correlations between esds in cell parameters are only used when they are defined by crystal symmetry. An approximate (isotropic) treatment of cell esds is used for estimating esds involving l.s. planes.

Refinement._olex2_refinement_description

1. Fixed Uiso At 1.2 times of: All $\mathrm{C}(\mathrm{H})$ groups, $\mathrm{All} \mathrm{C}(\mathrm{H}, \mathrm{H})$ groups At 1.5 times of: $\mathrm{All} \mathrm{C}(\mathrm{H}, \mathrm{H}, \mathrm{H})$ groups 2.a Ternary $\mathrm{CH}$ refined with riding coordinates: $\mathrm{C} 25(\mathrm{H} 25)$ 2.b Secondary $\mathrm{CH} 2$ refined with riding coordinates: C26(H26A,H26B) 2.c Aromatic/amide $\mathrm{H}$ refined with riding coordinates: C3(H3), C4(H4), C5(H5), C6(H6), C7(H7), C8(H8), C9(H9), C10(H10), C11(H11), C17(H17), C18(H18), C19(H19), C20(H20), C21(H21), C22(H22), C23(H23), C24(H24), C28(H28), C29(H29), C30(H30), C31(H31), C32(H32), C35(H35), C36(H36), C37(H37), C38(H38), C39(H39) 2.d $\mathrm{X}=\mathrm{CH} 2$ refined with riding coordinates: $\mathrm{C} 12(\mathrm{H} 12 \mathrm{~A}, \mathrm{H} 12 \mathrm{~B})$ 2.e Idealised Me refined as rotating group: C33(H33A,H33B,H33C)

Fractional atomic coordinates and isotropic or equivalent isotropic displacement parameters $\left(A^{2}\right)$

\begin{tabular}{lllll}
\hline & $x$ & $y$ & $z$ & $U_{\text {iso }} * / U_{\text {eq }}$ \\
\hline Fe1 & $0.51417(3)$ & $0.51824(3)$ & $0.44598(3)$ & $0.02729(12)$ \\
Fe2 & $0.49276(3)$ & $0.50436(3)$ & $0.09718(3)$ & $0.03059(12)$ \\
S1 & $0.48101(8)$ & $0.71100(6)$ & $0.26305(5)$ & $0.0377(2)$ \\
P1 & $0.41368(7)$ & $0.58912(6)$ & $0.27301(5)$ & $0.02534(18)$ \\
N1 & $0.1929(2)$ & $0.6660(2)$ & $0.13393(18)$ & $0.0313(7)$ \\
H1 & $0.184(3)$ & $0.638(3)$ & $0.177(2)$ & $0.060(14)^{*}$ \\
C1 & $0.4824(2)$ & $0.5067(2)$ & $0.33292(17)$ & $0.0258(6)$ \\
C2 & $0.5979(2)$ & $0.4958(2)$ & $0.34693(18)$ & $0.0289(7)$ \\
C3 & $0.6093(3)$ & $0.4193(2)$ & $0.3971(2)$ & $0.0336(8)$ \\
H3 & 0.676050 & 0.396826 & 0.416752 & $0.040 *$ \\
C4 & $0.5060(3)$ & $0.3813(2)$ & $0.41360(18)$ & $0.0340(8)$ \\
H4 & 0.491864 & 0.329266 & 0.445371 & $0.041^{*}$ \\
C5 & $0.4278(3)$ & $0.4347(2)$ & $0.37428(19)$ & $0.0313(8)$
\end{tabular}




\begin{tabular}{|c|c|c|c|c|}
\hline H5 & 0.351880 & 0.424507 & 0.375110 & $0.038^{*}$ \\
\hline $\mathrm{C} 6$ & $0.5020(3)$ & $0.6536(2)$ & $0.4784(2)$ & $0.0362(8)$ \\
\hline H6 & 0.495646 & 0.705091 & 0.444826 & $0.043^{*}$ \\
\hline $\mathrm{C} 7$ & $0.6001(3)$ & $0.6139(3)$ & $0.5061(2)$ & $0.0433(10)$ \\
\hline H7 & 0.671095 & 0.634692 & 0.494224 & $0.052^{*}$ \\
\hline $\mathrm{C} 8$ & $0.5741(3)$ & $0.5386(3)$ & $0.5541(2)$ & $0.0461(10)$ \\
\hline H8 & 0.624183 & 0.499956 & 0.580145 & $0.055^{*}$ \\
\hline C9 & $0.4597(3)$ & 0.5308 (3) & 0.5564 (2) & $0.0400(9)$ \\
\hline H9 & 0.419534 & 0.485660 & 0.584004 & $0.048^{*}$ \\
\hline $\mathrm{C} 10$ & $0.4162(3)$ & $0.6020(3)$ & $0.5104(2)$ & $0.0338(8)$ \\
\hline H10 & 0.341391 & 0.613355 & 0.502286 & $0.041^{*}$ \\
\hline $\mathrm{C} 11$ & $0.6860(3)$ & $0.5532(3)$ & $0.3191(2)$ & $0.0372(9)$ \\
\hline H11 & 0.669330 & 0.615155 & 0.304948 & $0.045^{*}$ \\
\hline C12 & $0.7875(3)$ & $0.5258(3)$ & $0.3120(2)$ & $0.0468(10)$ \\
\hline H12A & 0.807253 & 0.464396 & 0.325579 & $0.056^{*}$ \\
\hline H12B & 0.840713 & 0.567579 & 0.293300 & $0.056^{*}$ \\
\hline C15 & $0.3901(2)$ & $0.5270(2)$ & $0.18474(18)$ & $0.0243(7)$ \\
\hline $\mathrm{C} 16$ & 0.3439 (2) & $0.5629(2)$ & $0.11501(19)$ & $0.0258(7)$ \\
\hline $\mathrm{C} 17$ & $0.3356(2)$ & $0.4871(3)$ & $0.0635(2)$ & $0.0311(8)$ \\
\hline H17 & 0.308584 & 0.490242 & 0.012721 & $0.037^{*}$ \\
\hline C18 & $0.3742(3)$ & $0.4056(3)$ & 0.1005 (2) & $0.0334(8)$ \\
\hline H18 & 0.377109 & 0.345339 & 0.078782 & $0.040^{*}$ \\
\hline $\mathrm{C} 19$ & $0.4076(2)$ & $0.4301(2)$ & $0.1753(2)$ & $0.0284(7)$ \\
\hline H19 & 0.436519 & 0.389158 & 0.212655 & $0.034^{*}$ \\
\hline $\mathrm{C} 20$ & $0.6375(3)$ & $0.5637(3)$ & $0.1238(2)$ & $0.0486(11)$ \\
\hline $\mathrm{H} 20$ & 0.654219 & 0.596179 & 0.169639 & $0.058^{*}$ \\
\hline $\mathrm{C} 21$ & 0.5948 (3) & 0.6037 (3) & 0.0558 (2) & $0.0473(10)$ \\
\hline $\mathrm{H} 21$ & 0.577820 & 0.667188 & 0.048183 & $0.057^{*}$ \\
\hline $\mathrm{C} 22$ & $0.5822(3)$ & $0.5320(3)$ & $0.0016(2)$ & 0.0481 (11) \\
\hline $\mathrm{H} 22$ & 0.555074 & 0.538751 & -0.048898 & $0.058^{*}$ \\
\hline $\mathrm{C} 23$ & $0.6172(3)$ & 0.4478 (3) & 0.0359 (3) & $0.0511(12)$ \\
\hline $\mathrm{H} 23$ & 0.618086 & 0.388488 & 0.012367 & $0.061 *$ \\
\hline $\mathrm{C} 24$ & 0.6507 (3) & $0.4688(4)$ & 0.1119 (3) & $0.0560(12)$ \\
\hline $\mathrm{H} 24$ & 0.677411 & 0.425565 & 0.148199 & $0.067 *$ \\
\hline $\mathrm{C} 25$ & $0.3034(2)$ & 0.6599 (2) & $0.10146(19)$ & $0.0285(7)$ \\
\hline $\mathrm{H} 25$ & 0.351659 & 0.704876 & 0.128471 & $0.034 *$ \\
\hline $\mathrm{C} 26$ & $0.1612(3)$ & $0.7610(3)$ & 0.1533 (2) & $0.0360(9)$ \\
\hline $\mathrm{H} 26 \mathrm{~A}$ & 0.166909 & 0.800523 & 0.107220 & $0.043^{*}$ \\
\hline H26B & 0.211259 & 0.785864 & 0.192468 & $0.043^{*}$ \\
\hline $\mathrm{C} 27$ & $0.0461(3)$ & 0.7647 (2) & 0.1837 (2) & $0.0310(8)$ \\
\hline $\mathrm{C} 28$ & 0.0208 (3) & $0.8118(3)$ & $0.2500(2)$ & $0.0403(8)$ \\
\hline $\mathrm{H} 28$ & 0.076341 & 0.842763 & 0.277464 & $0.048 *$ \\
\hline $\mathrm{C} 29$ & $-0.0843(4)$ & $0.8144(3)$ & 0.2769 (2) & $0.0494(11)$ \\
\hline H29 & -0.100011 & 0.845362 & 0.323587 & $0.059^{*}$ \\
\hline $\mathrm{C} 30$ & $-0.1662(3)$ & 0.7725 (3) & 0.2365 (2) & $0.0455(10)$ \\
\hline $\mathrm{H} 30$ & -0.238792 & 0.775936 & 0.254158 & $0.055^{*}$ \\
\hline C31 & $-0.1420(3)$ & 0.7255 (3) & $0.1700(2)$ & $0.0439(10)$ \\
\hline H31 & -0.198078 & 0.696254 & 0.141809 & $0.053 *$ \\
\hline
\end{tabular}




\begin{tabular}{lllll} 
C32 & $-0.0363(3)$ & $0.7208(3)$ & $0.1443(2)$ & $0.0371(8)$ \\
H32 & -0.020110 & 0.686947 & 0.099091 & $0.045^{*}$ \\
C33 & $0.2996(3)$ & $0.6840(3)$ & $0.0164(2)$ & $0.0393(9)$ \\
H33A & 0.246891 & 0.643902 & -0.009349 & $0.059^{*}$ \\
H33B & 0.371270 & 0.674540 & -0.006238 & $0.059^{*}$ \\
H33C & 0.278049 & 0.748884 & 0.010187 & $0.059^{*}$ \\
C34 & $0.2781(3)$ & $0.5969(2)$ & $0.31390(19)$ & $0.0282(7)$ \\
C35 & $0.2483(3)$ & $0.6744(3)$ & $0.3560(2)$ & $0.0355(8)$ \\
H35 & 0.299204 & 0.722567 & 0.364829 & $0.043^{*}$ \\
C36 & $0.1443(3)$ & $0.6813(3)$ & $0.3852(2)$ & $0.0447(10)$ \\
H36 & 0.124415 & 0.733674 & 0.414952 & $0.054^{*}$ \\
C37 & $0.0706(3)$ & $0.6131(3)$ & $0.3711(2)$ & $0.0474(10)$ \\
H37 & -0.001096 & 0.619178 & 0.390135 & $0.057^{*}$ \\
C38 & $0.0984(3)$ & $0.5360(3)$ & $0.3299(2)$ & $0.0416(9)$ \\
H38 & 0.046658 & 0.488446 & 0.321133 & $0.050^{*}$ \\
C39 & $0.2026(3)$ & $0.5277(3)$ & $0.3009(2)$ & $0.0321(8)$ \\
H39 & 0.222188 & 0.474427 & 0.272187 & $0.039^{*}$ \\
\hline
\end{tabular}

Atomic displacement parameters $\left(\AA^{2}\right)$

\begin{tabular}{lllllll}
\hline & $U^{11}$ & $U^{22}$ & $U^{33}$ & $U^{12}$ & $U^{13}$ & $U^{23}$ \\
\hline Fe1 & $0.0245(2)$ & $0.0255(2)$ & $0.0319(2)$ & $0.0007(2)$ & $-0.00393(19)$ & $-0.00231(19)$ \\
Fe2 & $0.0203(2)$ & $0.0359(3)$ & $0.0356(2)$ & $0.0005(2)$ & $0.00347(18)$ & $-0.0043(2)$ \\
S1 & $0.0400(5)$ & $0.0212(4)$ & $0.0519(5)$ & $-0.0088(4)$ & $-0.0131(4)$ & $0.0047(4)$ \\
P1 & $0.0242(4)$ & $0.0192(4)$ & $0.0326(4)$ & $-0.0014(3)$ & $-0.0036(3)$ & $-0.0008(4)$ \\
N1 & $0.0276(14)$ & $0.0259(17)$ & $0.0403(18)$ & $0.0052(13)$ & $0.0000(13)$ & $0.0024(14)$ \\
C1 & $0.0272(14)$ & $0.0196(15)$ & $0.0305(15)$ & $-0.0003(15)$ & $-0.0023(12)$ & $-0.0002(12)$ \\
C2 & $0.0272(14)$ & $0.0250(18)$ & $0.0343(17)$ & $0.0025(15)$ & $0.0000(13)$ & $0.0010(14)$ \\
C3 & $0.0353(18)$ & $0.0278(19)$ & $0.0376(19)$ & $0.0075(16)$ & $0.0001(15)$ & $0.0034(16)$ \\
C4 & $0.047(2)$ & $0.0213(16)$ & $0.0339(17)$ & $0.0035(17)$ & $-0.0012(17)$ & $0.0012(13)$ \\
C5 & $0.0333(18)$ & $0.0286(19)$ & $0.0320(18)$ & $-0.0049(15)$ & $-0.0033(14)$ & $-0.0030(15)$ \\
C6 & $0.0340(19)$ & $0.0281(18)$ & $0.0464(19)$ & $0.0004(17)$ & $-0.0032(17)$ & $-0.0108(15)$ \\
C7 & $0.0292(18)$ & $0.043(2)$ & $0.058(3)$ & $-0.0043(19)$ & $-0.0082(17)$ & $-0.016(2)$ \\
C8 & $0.047(2)$ & $0.053(3)$ & $0.039(2)$ & $0.012(2)$ & $-0.0177(18)$ & $-0.013(2)$ \\
C9 & $0.043(2)$ & $0.044(2)$ & $0.0329(18)$ & $0.0008(18)$ & $-0.0030(16)$ & $-0.0030(18)$ \\
C10 & $0.0275(16)$ & $0.036(2)$ & $0.0383(19)$ & $0.0049(17)$ & $-0.0012(15)$ & $-0.0118(16)$ \\
C11 & $0.0309(18)$ & $0.040(2)$ & $0.040(2)$ & $0.0012(17)$ & $-0.0055(16)$ & $0.0067(17)$ \\
C12 & $0.0333(18)$ & $0.051(3)$ & $0.057(2)$ & $0.0004(19)$ & $0.0001(17)$ & $0.015(2)$ \\
C15 & $0.0158(13)$ & $0.0224(17)$ & $0.0347(17)$ & $-0.0013(13)$ & $0.0016(12)$ & $-0.0009(14)$ \\
C16 & $0.0193(14)$ & $0.0256(18)$ & $0.0325(18)$ & $-0.0012(13)$ & $0.0009(13)$ & $-0.0015(15)$ \\
C17 & $0.0201(13)$ & $0.038(2)$ & $0.0350(18)$ & $-0.0026(15)$ & $-0.0005(12)$ & $-0.0083(17)$ \\
C18 & $0.0253(15)$ & $0.031(2)$ & $0.044(2)$ & $-0.0024(15)$ & $0.0028(15)$ & $-0.0128(17)$ \\
C19 & $0.0230(15)$ & $0.0226(18)$ & $0.0396(19)$ & $0.0046(14)$ & $0.0010(14)$ & $-0.0018(14)$ \\
C20 & $0.0262(19)$ & $0.070(3)$ & $0.049(3)$ & $-0.016(2)$ & $0.0054(17)$ & $-0.010(2)$ \\
C21 & $0.0307(19)$ & $0.055(3)$ & $0.056(2)$ & $-0.0146(19)$ & $0.0130(19)$ & $-0.005(2)$ \\
C22 & $0.0316(18)$ & $0.070(3)$ & $0.042(2)$ & $-0.005(2)$ & $0.0108(16)$ & $-0.002(2)$ \\
C23 & $0.0279(18)$ & $0.065(3)$ & $0.061(3)$ & $0.008(2)$ & $0.0154(18)$ & $-0.016(2)$ \\
C24 & $0.0203(16)$ & $0.087(4)$ & $0.061(3)$ & $0.011(2)$ & $0.0045(17)$ & $0.007(3)$ \\
& & & & &
\end{tabular}




$\begin{array}{lllllll}\text { C25 } & 0.0257(15) & 0.0284(18) & 0.0313(17) & 0.0000(14) & -0.0020(14) & 0.0015(14) \\ \text { C26 } & 0.0325(18) & 0.029(2) & 0.047(2) & 0.0037(16) & -0.0019(16) & 0.0004(17) \\ \text { C27 } & 0.0322(17) & 0.0251(18) & 0.0358(18) & 0.0075(15) & -0.0035(15) & 0.0037(15) \\ \text { C28 } & 0.048(2) & 0.0313(19) & 0.0417(19) & 0.0061(19) & -0.0051(18) & -0.0029(15) \\ \text { C29 } & 0.068(3) & 0.041(2) & 0.039(2) & 0.015(2) & 0.013(2) & 0.0002(19) \\ \text { C30 } & 0.043(2) & 0.036(2) & 0.057(3) & 0.0111(19) & 0.016(2) & 0.010(2) \\ \text { C31 } & 0.036(2) & 0.043(3) & 0.053(3) & -0.0037(18) & 0.0004(18) & 0.005(2) \\ \text { C32 } & 0.0368(18) & 0.038(2) & 0.0362(18) & 0.0001(17) & 0.0009(15) & 0.0016(16) \\ \text { C33 } & 0.044(2) & 0.040(2) & 0.034(2) & 0.0032(19) & -0.0003(17) & 0.0050(17) \\ \text { C34 } & 0.0274(16) & 0.0282(18) & 0.0290(16) & 0.0091(15) & -0.0038(13) & -0.0037(14) \\ \text { C35 } & 0.0398(19) & 0.0301(19) & 0.0366(19) & 0.0072(17) & -0.0090(16) & -0.0061(15) \\ \text { C36 } & 0.050(2) & 0.041(2) & 0.042(2) & 0.024(2) & 0.0029(18) & -0.0110(18) \\ \text { C37 } & 0.036(2) & 0.059(3) & 0.047(2) & 0.016(2) & 0.0116(18) & -0.001(2) \\ \text { C38 } & 0.0312(18) & 0.049(3) & 0.045(2) & 0.0029(19) & 0.0069(16) & 0.0023(18) \\ \text { C39 } & 0.0302(16) & 0.029(2) & 0.0368(19) & 0.0034(16) & 0.0035(14) & -0.0016(15) \\ & & & & & \end{array}$

Geometric parameters $\left(A,{ }^{\circ}\right)$

\begin{tabular}{llll}
\hline $\mathrm{Fe} 1-\mathrm{C} 1$ & $2.022(3)$ & $\mathrm{C} 15-\mathrm{C} 16$ & $1.442(5)$ \\
$\mathrm{Fe} 1-\mathrm{C} 2$ & $2.043(3)$ & $\mathrm{C} 15-\mathrm{C} 19$ & $1.425(4)$ \\
$\mathrm{Fe} 1-\mathrm{C} 3$ & $2.038(3)$ & $\mathrm{C} 16-\mathrm{C} 17$ & $1.420(5)$ \\
$\mathrm{Fe} 1-\mathrm{C} 4$ & $2.059(3)$ & $\mathrm{C} 16-\mathrm{C} 25$ & $1.506(5)$ \\
$\mathrm{Fe} 1-\mathrm{C} 5$ & $2.041(3)$ & $\mathrm{C} 17-\mathrm{H} 17$ & 0.9500 \\
$\mathrm{Fe} 1-\mathrm{C} 6$ & $2.040(3)$ & $\mathrm{C} 17-\mathrm{C} 18$ & $1.425(5)$ \\
$\mathrm{Fe} 1-\mathrm{C} 7$ & $2.034(4)$ & $\mathrm{C} 18-\mathrm{H} 18$ & 0.9500 \\
$\mathrm{Fe} 1-\mathrm{C} 8$ & $2.050(4)$ & $\mathrm{C} 18-\mathrm{C} 19$ & $1.416(5)$ \\
$\mathrm{Fe} 1-\mathrm{C} 9$ & $2.052(4)$ & $\mathrm{C} 19-\mathrm{H} 19$ & 0.9500 \\
$\mathrm{Fe} 1-\mathrm{C} 10$ & $2.048(3)$ & $\mathrm{C} 20-\mathrm{H} 20$ & 0.9500 \\
$\mathrm{Fe} 2-\mathrm{C} 15$ & $2.015(3)$ & $\mathrm{C} 20-\mathrm{C} 21$ & $1.423(6)$ \\
$\mathrm{Fe} 2-\mathrm{C} 16$ & $2.048(3)$ & $\mathrm{C} 20-\mathrm{C} 24$ & $1.396(6)$ \\
$\mathrm{Fe} 2-\mathrm{C} 17$ & $2.045(3)$ & $\mathrm{C} 21-\mathrm{H} 21$ & 0.9500 \\
$\mathrm{Fe} 2-\mathrm{C} 18$ & $2.045(3)$ & $\mathrm{C} 21-\mathrm{C} 22$ & $1.411(6)$ \\
$\mathrm{Fe} 2-\mathrm{C} 19$ & $2.031(3)$ & $\mathrm{C} 22-\mathrm{H} 22$ & 0.9500 \\
$\mathrm{Fe} 2-\mathrm{C} 20$ & $2.038(4)$ & $\mathrm{C} 22-\mathrm{C} 23$ & $1.421(6)$ \\
$\mathrm{Fe} 2-\mathrm{C} 21$ & $2.042(4)$ & $\mathrm{C} 23-\mathrm{H} 23$ & 0.9500 \\
$\mathrm{Fe} 2-\mathrm{C} 22$ & $2.042(4)$ & $\mathrm{C} 23-\mathrm{C} 24$ & $1.424(6)$ \\
$\mathrm{Fe} 2-\mathrm{C} 23$ & $2.044(4)$ & $\mathrm{C} 24-\mathrm{H} 24$ & 0.9500 \\
$\mathrm{Fe} 2-\mathrm{C} 24$ & $2.034(4)$ & $\mathrm{C} 25-\mathrm{H} 25$ & 1.0000 \\
$\mathrm{~S} 1-\mathrm{P} 1$ & $1.9538(12)$ & $\mathrm{C} 25-\mathrm{C} 33$ & $1.528(5)$ \\
$\mathrm{P} 1-\mathrm{C} 1$ & $1.798(3)$ & $\mathrm{C} 26-\mathrm{H} 26 \mathrm{~A}$ & 0.9900 \\
$\mathrm{P} 1-\mathrm{C} 15$ & $1.808(3)$ & $\mathrm{C} 26-\mathrm{H} 26 \mathrm{~B}$ & 0.9900 \\
$\mathrm{P} 1-\mathrm{C} 34$ & $1.826(3)$ & $\mathrm{C} 26-\mathrm{C} 27$ & $1.518(5)$ \\
$\mathrm{N} 1-\mathrm{H} 1$ & $0.87(4)$ & $\mathrm{C} 27-\mathrm{C} 28$ & $1.379(5)$ \\
$\mathrm{N} 1-\mathrm{C} 25$ & $1.481(4)$ & $\mathrm{C} 27-\mathrm{C} 32$ & $1.383(5)$ \\
$\mathrm{N} 1-\mathrm{C} 26$ & $1.466(4)$ & $\mathrm{C} 28-\mathrm{H} 28$ & 0.9500 \\
$\mathrm{C} 1-\mathrm{C} 2$ & $1.458(4)$ & $\mathrm{C} 28-\mathrm{C} 29$ & 0.9500 \\
$\mathrm{C} 1-\mathrm{C} 5$ & $1.435(4)$ & $\mathrm{C} 29-\mathrm{H} 29$ & $1.375(6)$ \\
$\mathrm{C} 2-\mathrm{C} 3$ & $1.417(5)$ & $\mathrm{C} 29-\mathrm{C} 30$ &
\end{tabular}




\begin{tabular}{|c|c|c|c|}
\hline $\mathrm{C} 2-\mathrm{C} 11$ & $1.452(5)$ & $\mathrm{C} 30-\mathrm{H} 30$ & 0.9500 \\
\hline $\mathrm{C} 3-\mathrm{H} 3$ & 0.9500 & $\mathrm{C} 30-\mathrm{C} 31$ & $1.378(6)$ \\
\hline $\mathrm{C} 3-\mathrm{C} 4$ & $1.420(5)$ & $\mathrm{C} 31-\mathrm{H} 31$ & 0.9500 \\
\hline $\mathrm{C} 4-\mathrm{H} 4$ & 0.9500 & $\mathrm{C} 31-\mathrm{C} 32$ & $1.383(5)$ \\
\hline $\mathrm{C} 4-\mathrm{C} 5$ & $1.414(5)$ & $\mathrm{C} 32-\mathrm{H} 32$ & 0.9500 \\
\hline $\mathrm{C} 5-\mathrm{H} 5$ & 0.9500 & $\mathrm{C} 33-\mathrm{H} 33 \mathrm{~A}$ & 0.9800 \\
\hline C6-H6 & 0.9500 & C $33-\mathrm{H} 33 \mathrm{~B}$ & 0.9800 \\
\hline $\mathrm{C} 6-\mathrm{C} 7$ & $1.425(5)$ & $\mathrm{C} 33-\mathrm{H} 33 \mathrm{C}$ & 0.9800 \\
\hline $\mathrm{C} 6-\mathrm{C} 10$ & $1.412(5)$ & C34-C35 & $1.388(5)$ \\
\hline $\mathrm{C} 7-\mathrm{H} 7$ & 0.9500 & $\mathrm{C} 34-\mathrm{C} 39$ & $1.385(5)$ \\
\hline $\mathrm{C} 7-\mathrm{C} 8$ & $1.410(6)$ & $\mathrm{C} 35-\mathrm{H} 35$ & 0.9500 \\
\hline $\mathrm{C} 8-\mathrm{H} 8$ & 0.9500 & $\mathrm{C} 35-\mathrm{C} 36$ & $1.386(5)$ \\
\hline $\mathrm{C} 8-\mathrm{C} 9$ & $1.418(5)$ & $\mathrm{C} 36-\mathrm{H} 36$ & 0.9500 \\
\hline C9-H9 & 0.9500 & $\mathrm{C} 36-\mathrm{C} 37$ & $1.365(6)$ \\
\hline $\mathrm{C} 9-\mathrm{C} 10$ & $1.412(5)$ & C37-H37 & 0.9500 \\
\hline $\mathrm{C} 10-\mathrm{H} 10$ & 0.9500 & $\mathrm{C} 37-\mathrm{C} 38$ & $1.370(5)$ \\
\hline $\mathrm{C} 11-\mathrm{H} 11$ & 0.9500 & $\mathrm{C} 38-\mathrm{H} 38$ & 0.9500 \\
\hline $\mathrm{C} 11-\mathrm{C} 12$ & $1.321(5)$ & C38-C39 & $1.389(5)$ \\
\hline $\mathrm{C} 12-\mathrm{H} 12 \mathrm{~A}$ & 0.9500 & C39- H39 & 0.9500 \\
\hline $\mathrm{C} 12-\mathrm{H} 12 \mathrm{~B}$ & 0.9500 & & \\
\hline $\mathrm{C} 1-\mathrm{Fe} 1-\mathrm{C} 2$ & $42.02(12)$ & $\mathrm{C} 8-\mathrm{C} 7-\mathrm{H} 7$ & 125.7 \\
\hline $\mathrm{C} 1-\mathrm{Fe} 1-\mathrm{C} 3$ & $69.20(13)$ & $\mathrm{Fe} 1-\mathrm{C} 8-\mathrm{H} 8$ & 126.4 \\
\hline $\mathrm{C} 1-\mathrm{Fe} 1-\mathrm{C} 4$ & $69.07(12)$ & $\mathrm{C} 7-\mathrm{C} 8-\mathrm{Fe} 1$ & $69.2(2)$ \\
\hline $\mathrm{C} 1-\mathrm{Fe} 1-\mathrm{C} 5$ & $41.37(12)$ & $\mathrm{C} 7-\mathrm{C} 8-\mathrm{H} 8$ & 126.1 \\
\hline $\mathrm{C} 1-\mathrm{Fe} 1-\mathrm{C} 6$ & $109.64(14)$ & $\mathrm{C} 7-\mathrm{C} 8-\mathrm{C} 9$ & $107.8(4)$ \\
\hline $\mathrm{C} 1-\mathrm{Fe} 1-\mathrm{C} 7$ & $131.46(16)$ & $\mathrm{C} 9-\mathrm{C} 8-\mathrm{Fe} 1$ & $69.9(2)$ \\
\hline $\mathrm{C} 1-\mathrm{Fe} 1-\mathrm{C} 8$ & $169.32(15)$ & $\mathrm{C} 9-\mathrm{C} 8-\mathrm{H} 8$ & 126.1 \\
\hline $\mathrm{C} 1-\mathrm{Fe} 1-\mathrm{C} 9$ & $149.64(13)$ & $\mathrm{Fe} 1-\mathrm{C} 9-\mathrm{H} 9$ & 126.1 \\
\hline $\mathrm{C} 1-\mathrm{Fe} 1-\mathrm{C} 10$ & $118.11(13)$ & $\mathrm{C} 8-\mathrm{C} 9-\mathrm{Fe} 1$ & $69.7(2)$ \\
\hline $\mathrm{C} 2-\mathrm{Fe} 1-\mathrm{C} 4$ & $68.88(13)$ & $\mathrm{C} 8-\mathrm{C} 9-\mathrm{H} 9$ & 126.1 \\
\hline $\mathrm{C} 2-\mathrm{Fe} 1-\mathrm{C} 8$ & $128.36(14)$ & $\mathrm{C} 10-\mathrm{C} 9-\mathrm{Fe} 1$ & $69.7(2)$ \\
\hline $\mathrm{C} 2-\mathrm{Fe} 1-\mathrm{C} 9$ & $167.74(13)$ & $\mathrm{C} 10-\mathrm{C} 9-\mathrm{C} 8$ & $107.7(4)$ \\
\hline $\mathrm{C} 2-\mathrm{Fe} 1-\mathrm{C} 10$ & $149.20(14)$ & $\mathrm{C} 10-\mathrm{C} 9-\mathrm{H} 9$ & 126.1 \\
\hline $\mathrm{C} 3-\mathrm{Fe} 1-\mathrm{C} 2$ & $40.65(13)$ & $\mathrm{Fe} 1-\mathrm{C} 10-\mathrm{H} 10$ & 126.5 \\
\hline $\mathrm{C} 3-\mathrm{Fe} 1-\mathrm{C} 4$ & $40.54(13)$ & $\mathrm{C} 6-\mathrm{C} 10-\mathrm{Fe} 1$ & $69.51(19)$ \\
\hline $\mathrm{C} 3-\mathrm{Fe} 1-\mathrm{C} 5$ & $68.28(14)$ & $\mathrm{C} 6-\mathrm{C} 10-\mathrm{C} 9$ & $108.9(3)$ \\
\hline $\mathrm{C} 3-\mathrm{Fe} 1-\mathrm{C} 6$ & $146.12(14)$ & $\mathrm{C} 6-\mathrm{C} 10-\mathrm{H} 10$ & 125.5 \\
\hline $\mathrm{C} 3-\mathrm{Fe} 1-\mathrm{C} 8$ & $106.16(15)$ & $\mathrm{C} 9-\mathrm{C} 10-\mathrm{Fe} 1$ & $70.0(2)$ \\
\hline $\mathrm{C} 3-\mathrm{Fe} 1-\mathrm{C} 9$ & $130.18(15)$ & $\mathrm{C} 9-\mathrm{C} 10-\mathrm{H} 10$ & 125.5 \\
\hline $\mathrm{C} 3-\mathrm{Fe} 1-\mathrm{C} 10$ & $170.14(15)$ & $\mathrm{C} 2-\mathrm{C} 11-\mathrm{H} 11$ & 117.6 \\
\hline $\mathrm{C} 5-\mathrm{Fe} 1-\mathrm{C} 2$ & $69.56(13)$ & $\mathrm{C} 12-\mathrm{C} 11-\mathrm{C} 2$ & $124.9(4)$ \\
\hline $\mathrm{C} 5-\mathrm{Fe} 1-\mathrm{C} 4$ & 40.34 (13) & $\mathrm{C} 12-\mathrm{C} 11-\mathrm{H} 11$ & 117.6 \\
\hline $\mathrm{C} 5-\mathrm{Fe} 1-\mathrm{C} 8$ & $147.07(17)$ & $\mathrm{C} 11-\mathrm{C} 12-\mathrm{H} 12 \mathrm{~A}$ & 120.0 \\
\hline $\mathrm{C} 5-\mathrm{Fe} 1-\mathrm{C} 9$ & 117.27 (14) & $\mathrm{C} 11-\mathrm{C} 12-\mathrm{H} 12 \mathrm{~B}$ & 120.0 \\
\hline $\mathrm{C} 5-\mathrm{Fe} 1-\mathrm{C} 10$ & $112.19(14)$ & $\mathrm{H} 12 \mathrm{~A}-\mathrm{C} 12-\mathrm{H} 12 \mathrm{~B}$ & 120.0 \\
\hline $\mathrm{C} 6-\mathrm{Fe} 1-\mathrm{C} 2$ & $115.12(14)$ & $\mathrm{P} 1-\mathrm{C} 15-\mathrm{Fe} 2$ & $128.81(15)$ \\
\hline $\mathrm{C} 6-\mathrm{Fe} 1-\mathrm{C} 4$ & $172.97(14)$ & $\mathrm{C} 16-\mathrm{C} 15-\mathrm{Fe} 2$ & $70.45(18)$ \\
\hline
\end{tabular}




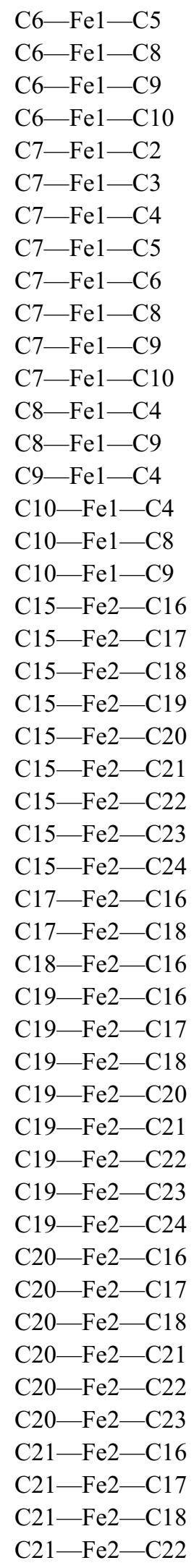

$134.31(14)$

68.49 (16)

$68.30(15)$

40.40 (14)

$106.31(15)$

$113.02(15)$

$145.02(15)$

$172.48(17)$

40.95 (14)

40.39 (17)

$68.02(16)$

67.90 (14)

$114.12(15)$

$40.46(15)$

$109.09(15)$

133.55 (14)

$67.79(15)$

40.27 (14)

$41.56(13)$

$68.88(13)$

$68.90(13)$

41.24 (12)

$108.12(15)$

$122.99(15)$

$158.52(17)$

$159.51(16)$

$123.33(16)$

40.60 (13)

40.78 (14)

$68.88(13)$

69.50 (13)

68.69 (14)

40.66 (13)

$121.55(15)$

$158.32(15)$

$159.04(16)$

$122.14(17)$

$106.22(17)$

$125.46(15)$

$161.99(17)$

$156.30(17)$

40.84 (17)

$68.27(17)$

$68.13(17)$

$108.60(15)$

$124.77(17)$

$160.40(16)$

40.43 (16)
C16-C15-P1

$\mathrm{C} 19-\mathrm{C} 15-\mathrm{Fe} 2$

$\mathrm{C} 19-\mathrm{C} 15-\mathrm{P} 1$

$\mathrm{C} 19-\mathrm{C} 15-\mathrm{C} 16$

$\mathrm{C} 15-\mathrm{C} 16-\mathrm{Fe} 2$

$\mathrm{C} 15-\mathrm{C} 16-\mathrm{C} 25$

$\mathrm{C} 17-\mathrm{C} 16-\mathrm{Fe} 2$

$\mathrm{C} 17-\mathrm{C} 16-\mathrm{C} 15$

$\mathrm{C} 17-\mathrm{C} 16-\mathrm{C} 25$

$\mathrm{C} 25-\mathrm{C} 16-\mathrm{Fe} 2$

$\mathrm{Fe} 2-\mathrm{C} 17-\mathrm{H} 17$

$\mathrm{C} 16-\mathrm{C} 17-\mathrm{Fe} 2$

$\mathrm{C} 16-\mathrm{C} 17-\mathrm{H} 17$

C16-C17-C18

$\mathrm{C} 18-\mathrm{C} 17-\mathrm{Fe} 2$

$\mathrm{C} 18-\mathrm{C} 17-\mathrm{H} 17$

$\mathrm{Fe} 2-\mathrm{C} 18-\mathrm{H} 18$

$\mathrm{C} 17-\mathrm{C} 18-\mathrm{Fe} 2$

C17-C18-H18

$\mathrm{C} 19-\mathrm{C} 18-\mathrm{Fe} 2$

$\mathrm{C} 19-\mathrm{C} 18-\mathrm{C} 17$

C19-C18-H18

$\mathrm{Fe} 2-\mathrm{C} 19-\mathrm{H} 19$

C15-C19-Fe2

C15-C19-H19

$\mathrm{C} 18-\mathrm{C} 19-\mathrm{Fe} 2$

$\mathrm{C} 18-\mathrm{C} 19-\mathrm{C} 15$

C18-C19-H19

$\mathrm{Fe} 2-\mathrm{C} 20-\mathrm{H} 20$

$\mathrm{C} 21-\mathrm{C} 20-\mathrm{Fe} 2$

$\mathrm{C} 21-\mathrm{C} 20-\mathrm{H} 20$

$\mathrm{C} 24-\mathrm{C} 20-\mathrm{Fe} 2$

$\mathrm{C} 24-\mathrm{C} 20-\mathrm{H} 20$

$\mathrm{C} 24-\mathrm{C} 20-\mathrm{C} 21$

$\mathrm{Fe} 2-\mathrm{C} 21-\mathrm{H} 21$

$\mathrm{C} 20-\mathrm{C} 21-\mathrm{Fe} 2$

$\mathrm{C} 20-\mathrm{C} 21-\mathrm{H} 21$

$\mathrm{C} 22-\mathrm{C} 21-\mathrm{Fe} 2$

$\mathrm{C} 22-\mathrm{C} 21-\mathrm{C} 20$

$\mathrm{C} 22-\mathrm{C} 21-\mathrm{H} 21$

$\mathrm{Fe} 2-\mathrm{C} 22-\mathrm{H} 22$

$\mathrm{C} 21-\mathrm{C} 22-\mathrm{Fe} 2$

$\mathrm{C} 21-\mathrm{C} 22-\mathrm{H} 22$

$\mathrm{C} 21-\mathrm{C} 22-\mathrm{C} 23$

$\mathrm{C} 23-\mathrm{C} 22-\mathrm{Fe} 2$

$\mathrm{C} 23-\mathrm{C} 22-\mathrm{H} 22$

$\mathrm{Fe} 2-\mathrm{C} 23-\mathrm{H} 23$

$\mathrm{C} 22-\mathrm{C} 23-\mathrm{Fe} 2$
$127.4(2)$

69.98 (19)

$124.1(3)$

108.4 (3)

67.99 (17)

$126.8(3)$

69.58 (17)

$106.7(3)$

$126.3(3)$

$131.2(2)$

126.6

69.82 (17)

125.5

108.9 (3)

69.61 (18)

125.5

126.9

69.62 (19)

126.0

69.13 (19)

108.1 (3)

126.0

126.5

68.79 (19)

126.1

$70.2(2)$

107.9 (3)

126.1

126.2

69.7 (2)

125.8

69.8 (2)

125.8

108.4 (4)

126.2

69.4 (2)

126.1

69.8 (2)

107.7 (4)

126.1

126.1

69.8 (2)

125.9

108.1 (4)

69.7 (2)

125.9

126.5

$69.6(2)$ 


\begin{tabular}{|c|c|}
\hline $\mathrm{C} 21-\mathrm{Fe} 2-\mathrm{C} 23$ & $68.27(18)$ \\
\hline $\mathrm{C} 22-\mathrm{Fe} 2-\mathrm{C} 16$ & $122.02(16)$ \\
\hline $\mathrm{C} 22-\mathrm{Fe} 2-\mathrm{C} 17$ & $107.63(15)$ \\
\hline $\mathrm{C} 22-\mathrm{Fe} 2-\mathrm{C} 18$ & $123.18(16)$ \\
\hline $\mathrm{C} 22-\mathrm{Fe} 2-\mathrm{C} 23$ & $40.70(17)$ \\
\hline $\mathrm{C} 23-\mathrm{Fe} 2-\mathrm{C} 16$ & $156.93(15)$ \\
\hline $\mathrm{C} 23-\mathrm{Fe} 2-\mathrm{C} 17$ & $121.02(16)$ \\
\hline $\mathrm{C} 23-\mathrm{Fe} 2-\mathrm{C} 18$ & $106.00(16)$ \\
\hline $\mathrm{C} 24-\mathrm{Fe} 2-\mathrm{C} 16$ & $161.19(17)$ \\
\hline $\mathrm{C} 24-\mathrm{Fe} 2-\mathrm{C} 17$ & $156.49(18)$ \\
\hline $\mathrm{C} 24-\mathrm{Fe} 2-\mathrm{C} 18$ & $120.53(19)$ \\
\hline $\mathrm{C} 24-\mathrm{Fe} 2-\mathrm{C} 20$ & 40.09 (17) \\
\hline $\mathrm{C} 24-\mathrm{Fe} 2-\mathrm{C} 21$ & 68.24 (19) \\
\hline $\mathrm{C} 24-\mathrm{Fe} 2-\mathrm{C} 22$ & $68.47(18)$ \\
\hline $\mathrm{C} 24-\mathrm{Fe} 2-\mathrm{C} 23$ & $40.86(17)$ \\
\hline $\mathrm{C} 1-\mathrm{P} 1-\mathrm{S} 1$ & $116.52(11)$ \\
\hline $\mathrm{C} 1-\mathrm{P} 1-\mathrm{C} 15$ & $104.19(15)$ \\
\hline $\mathrm{C} 1-\mathrm{P} 1-\mathrm{C} 34$ & $104.25(15)$ \\
\hline $\mathrm{C} 15-\mathrm{P} 1-\mathrm{S} 1$ & $116.06(11)$ \\
\hline $\mathrm{C} 15-\mathrm{P} 1-\mathrm{C} 34$ & $102.49(14)$ \\
\hline $\mathrm{C} 34-\mathrm{P} 1-\mathrm{S} 1$ & $111.75(12)$ \\
\hline $\mathrm{C} 25-\mathrm{N} 1-\mathrm{H} 1$ & $115(3)$ \\
\hline $\mathrm{C} 26-\mathrm{N} 1-\mathrm{H} 1$ & $102(3)$ \\
\hline $\mathrm{C} 26-\mathrm{N} 1-\mathrm{C} 25$ & $113.0(3)$ \\
\hline $\mathrm{P} 1-\mathrm{C} 1-\mathrm{Fe} 1$ & $127.36(17)$ \\
\hline $\mathrm{C} 2-\mathrm{C} 1-\mathrm{Fe} 1$ & $69.77(17)$ \\
\hline $\mathrm{C} 2-\mathrm{C} 1-\mathrm{P} 1$ & $129.2(2)$ \\
\hline $\mathrm{C} 5-\mathrm{C} 1-\mathrm{Fe} 1$ & $70.02(18)$ \\
\hline $\mathrm{C} 5-\mathrm{C} 1-\mathrm{P} 1$ & $123.5(2)$ \\
\hline $\mathrm{C} 5-\mathrm{C} 1-\mathrm{C} 2$ & 107.3 \\
\hline $\mathrm{C} 1-\mathrm{C} 2-\mathrm{Fe} 1$ & $68.21(17)$ \\
\hline $\mathrm{C} 3-\mathrm{C} 2-\mathrm{Fe} 1$ & 69.48 (19) \\
\hline $\mathrm{C} 3-\mathrm{C} 2-\mathrm{C} 1$ & $106.6(3)$ \\
\hline $\mathrm{C} 3-\mathrm{C} 2-\mathrm{C} 11$ & $125.3(3)$ \\
\hline $\mathrm{C} 11-\mathrm{C} 2-\mathrm{Fe} 1$ & $125.0(3)$ \\
\hline $\mathrm{C} 11-\mathrm{C} 2-\mathrm{C} 1$ & $128.0(3)$ \\
\hline $\mathrm{Fe} 1-\mathrm{C} 3-\mathrm{H} 3$ & 126.1 \\
\hline $\mathrm{C} 2-\mathrm{C} 3-\mathrm{Fe} 1$ & 69.87 (19) \\
\hline $\mathrm{C} 2-\mathrm{C} 3-\mathrm{H} 3$ & 125.1 \\
\hline $\mathrm{C} 2-\mathrm{C} 3-\mathrm{C} 4$ & $109.7(3)$ \\
\hline $\mathrm{C} 4-\mathrm{C} 3-\mathrm{Fe} 1$ & $70.52(19)$ \\
\hline $\mathrm{C} 4-\mathrm{C} 3-\mathrm{H} 3$ & 125.1 \\
\hline $\mathrm{Fe} 1-\mathrm{C} 4-\mathrm{H} 4$ & 127.4 \\
\hline $\mathrm{C} 3-\mathrm{C} 4-\mathrm{Fe} 1$ & $68.93(19)$ \\
\hline $\mathrm{C} 3-\mathrm{C} 4-\mathrm{H} 4$ & 126.1 \\
\hline $\mathrm{C} 5-\mathrm{C} 4-\mathrm{Fe} 1$ & 69.14 (19) \\
\hline $\mathrm{C} 5-\mathrm{C} 4-\mathrm{C} 3$ & $107.8(3)$ \\
\hline $\mathrm{C}_{5}-\mathrm{C}_{4}-\mathrm{H} 4$ & \\
\hline
\end{tabular}

$\mathrm{C} 22-\mathrm{C} 23-\mathrm{H} 23$

$\mathrm{C} 22-\mathrm{C} 23-\mathrm{C} 24 \quad 107.4$ (4)

$\mathrm{C} 24-\mathrm{C} 23-\mathrm{Fe} 2 \quad 69.2(2)$

$\mathrm{C} 24-\mathrm{C} 23-\mathrm{H} 23$

$\mathrm{Fe} 2-\mathrm{C} 24-\mathrm{H} 24$

$\mathrm{C} 20-\mathrm{C} 24-\mathrm{Fe} 2 \quad 70.1$ (2)

$\mathrm{C} 20-\mathrm{C} 24-\mathrm{C} 23 \quad 108.4$ (4)

$\mathrm{C} 20-\mathrm{C} 24-\mathrm{H} 24 \quad 125.8$

$\mathrm{C} 23-\mathrm{C} 24-\mathrm{Fe} 2 \quad 70.0$ (2)

$\mathrm{C} 23-\mathrm{C} 24-\mathrm{H} 24 \quad 125.8$

$\mathrm{N} 1-\mathrm{C} 25-\mathrm{C} 16 \quad 107.5$ (3)

$\mathrm{N} 1-\mathrm{C} 25-\mathrm{H} 25$

$\mathrm{N} 1-\mathrm{C} 25-\mathrm{C} 33$

$\mathrm{C} 16-\mathrm{C} 25-\mathrm{H} 25 \quad 109.3$

$\mathrm{C} 16-\mathrm{C} 25-\mathrm{C} 33 \quad 112.0$ (3)

$\mathrm{C} 33-\mathrm{C} 25-\mathrm{H} 25 \quad 109.3$

$\mathrm{N} 1-\mathrm{C} 26-\mathrm{H} 26 \mathrm{~A}$

$\mathrm{N} 1-\mathrm{C} 26-\mathrm{H} 26 \mathrm{~B}$

$\mathrm{N} 1-\mathrm{C} 26-\mathrm{C} 27 \quad 111.4$ (3)

$\mathrm{H} 26 \mathrm{~A}-\mathrm{C} 26-\mathrm{H} 26 \mathrm{~B} \quad 108.0$

$\mathrm{C} 27-\mathrm{C} 26-\mathrm{H} 26 \mathrm{~A} \quad 109.4$

$\mathrm{C} 27-\mathrm{C} 26-\mathrm{H} 26 \mathrm{~B} \quad 109.4$

$\mathrm{C} 28-\mathrm{C} 27-\mathrm{C} 26 \quad 121.6(3)$

$\mathrm{C} 28-\mathrm{C} 27-\mathrm{C} 32 \quad 118.5(3)$

$\mathrm{C} 32-\mathrm{C} 27-\mathrm{C} 26 \quad 120.0$ (3)

$\mathrm{C} 27-\mathrm{C} 28-\mathrm{H} 28 \quad 119.6$

$\mathrm{C} 27-\mathrm{C} 28-\mathrm{C} 29 \quad 120.9$ (4)

$\mathrm{C} 29-\mathrm{C} 28-\mathrm{H} 28$

$\mathrm{C} 28-\mathrm{C} 29-\mathrm{H} 29 \quad 119.9$

$\mathrm{C} 30-\mathrm{C} 29-\mathrm{C} 28 \quad 120.3$ (4)

$\mathrm{C} 30-\mathrm{C} 29-\mathrm{H} 29$

$\mathrm{C} 29-\mathrm{C} 30-\mathrm{H} 30 \quad 120.3$

$\mathrm{C} 29-\mathrm{C} 30-\mathrm{C} 31$

$\mathrm{C} 31-\mathrm{C} 30-\mathrm{H} 30 \quad 120.3$

$\mathrm{C} 30-\mathrm{C} 31-\mathrm{H} 31 \quad 119.9$

$\mathrm{C} 30-\mathrm{C} 31-\mathrm{C} 32$

$\mathrm{C} 32-\mathrm{C} 31-\mathrm{H} 31 \quad 119.9$

$\mathrm{C} 27-\mathrm{C} 32-\mathrm{C} 31 \quad 120.7$ (4)

$\mathrm{C} 27-\mathrm{C} 32-\mathrm{H} 32 \quad 119.6$

$\mathrm{C} 31-\mathrm{C} 32-\mathrm{H} 32 \quad 119.6$

$\mathrm{C} 25-\mathrm{C} 33-\mathrm{H} 33 \mathrm{~A} \quad 109.5$

$\mathrm{C} 25-\mathrm{C} 33-\mathrm{H} 33 \mathrm{~B} \quad 109.5$

$\mathrm{C} 25-\mathrm{C} 33-\mathrm{H} 33 \mathrm{C} \quad 109.5$

$\mathrm{H} 33 \mathrm{~A}-\mathrm{C} 33-\mathrm{H} 33 \mathrm{~B} \quad 109.5$

$\mathrm{H} 33 \mathrm{~A}-\mathrm{C} 33-\mathrm{H} 33 \mathrm{C} \quad 109.5$

$\mathrm{H} 33 \mathrm{~B}-\mathrm{C} 33-\mathrm{H} 33 \mathrm{C} \quad 109.5$

$\mathrm{C} 35-\mathrm{C} 34-\mathrm{P} 1 \quad 120.0(3)$

C39-C34-P1 120.6 (3) 


\begin{tabular}{|c|c|}
\hline $\mathrm{Fe} 1-\mathrm{C} 5-\mathrm{H} 5$ & 126.8 \\
\hline $\mathrm{C} 1-\mathrm{C} 5-\mathrm{Fe} 1$ & $68.61(18)$ \\
\hline $\mathrm{C} 1-\mathrm{C} 5-\mathrm{H} 5$ & 125.7 \\
\hline $\mathrm{C} 4-\mathrm{C} 5-\mathrm{Fe} 1$ & $70.52(19)$ \\
\hline $\mathrm{C} 4-\mathrm{C} 5-\mathrm{C} 1$ & $108.6(3)$ \\
\hline $\mathrm{C} 4-\mathrm{C} 5-\mathrm{H} 5$ & 125.7 \\
\hline $\mathrm{Fe} 1-\mathrm{C} 6-\mathrm{H} 6$ & 125.7 \\
\hline $\mathrm{C} 7-\mathrm{C} 6-\mathrm{Fe} 1$ & $69.3(2)$ \\
\hline $\mathrm{C} 7-\mathrm{C} 6-\mathrm{H} 6$ & 126.5 \\
\hline $\mathrm{C} 10-\mathrm{C} 6-\mathrm{Fe} 1$ & $70.1(2)$ \\
\hline $\mathrm{C} 10-\mathrm{C} 6-\mathrm{H} 6$ & 126.5 \\
\hline $\mathrm{C} 10-\mathrm{C} 6-\mathrm{C} 7$ & $107.0(3)$ \\
\hline $\mathrm{Fe} 1-\mathrm{C} 7-\mathrm{H} 7$ & 125.7 \\
\hline $\mathrm{C} 6-\mathrm{C} 7-\mathrm{Fe} 1$ & $69.8(2)$ \\
\hline $\mathrm{C} 6-\mathrm{C} 7-\mathrm{H} 7$ & 125.7 \\
\hline $\mathrm{C} 8-\mathrm{C} 7-\mathrm{Fe} 1$ & $70.4(2)$ \\
\hline $\mathrm{C} 8-\mathrm{C} 7-\mathrm{C} 6$ & $108.5(3)$ \\
\hline $\mathrm{Fe} 1-\mathrm{C} 1-\mathrm{C} 2-\mathrm{C} 3$ & $59.1(2)$ \\
\hline $\mathrm{Fe} 1-\mathrm{C} 1-\mathrm{C} 2-\mathrm{C} 11$ & $-118.1(4)$ \\
\hline $\mathrm{Fe} 1-\mathrm{C} 1-\mathrm{C} 5-\mathrm{C} 4$ & $-59.3(2)$ \\
\hline $\mathrm{Fe} 1-\mathrm{C} 2-\mathrm{C} 3-\mathrm{C} 4$ & $59.4(2)$ \\
\hline $\mathrm{Fe} 1-\mathrm{C} 2-\mathrm{C} 11-\mathrm{C} 12$ & $114.7(4)$ \\
\hline $\mathrm{Fe} 1-\mathrm{C} 3-\mathrm{C} 4-\mathrm{C} 5$ & $58.4(2)$ \\
\hline $\mathrm{Fe} 1-\mathrm{C} 4-\mathrm{C} 5-\mathrm{C} 1$ & $58.2(2)$ \\
\hline $\mathrm{Fe} 1-\mathrm{C} 6-\mathrm{C} 7-\mathrm{C} 8$ & $-59.9(3)$ \\
\hline $\mathrm{Fe} 1-\mathrm{C} 6-\mathrm{C} 10-\mathrm{C} 9$ & $59.1(3)$ \\
\hline $\mathrm{Fe} 1-\mathrm{C} 7-\mathrm{C} 8-\mathrm{C} 9$ & $-59.4(3)$ \\
\hline $\mathrm{Fe} 1-\mathrm{C} 8-\mathrm{C} 9-\mathrm{C} 10$ & $-59.6(3)$ \\
\hline $\mathrm{Fe} 1-\mathrm{C} 9-\mathrm{C} 10-\mathrm{C} 6$ & $-58.8(2)$ \\
\hline $\mathrm{Fe} 2-\mathrm{C} 15-\mathrm{C} 16-\mathrm{C} 17$ & $59.1(2)$ \\
\hline $\mathrm{Fe} 2-\mathrm{C} 15-\mathrm{C} 16-\mathrm{C} 25$ & $-125.8(3)$ \\
\hline $\mathrm{Fe} 2-\mathrm{C} 15-\mathrm{C} 19-\mathrm{C} 18$ & $-59.5(2)$ \\
\hline $\mathrm{Fe} 2-\mathrm{C} 16-\mathrm{C} 17-\mathrm{C} 18$ & $58.8(2)$ \\
\hline $\mathrm{Fe} 2-\mathrm{C} 16-\mathrm{C} 25-\mathrm{N} 1$ & $-172.9(2)$ \\
\hline $\mathrm{Fe} 2-\mathrm{C} 16-\mathrm{C} 25-\mathrm{C} 33$ & $66.9(4)$ \\
\hline $\mathrm{Fe} 2-\mathrm{C} 17-\mathrm{C} 18-\mathrm{C} 19$ & $58.6(2)$ \\
\hline $\mathrm{Fe} 2-\mathrm{C} 18-\mathrm{C} 19-\mathrm{C} 15$ & $58.6(2)$ \\
\hline $\mathrm{Fe} 2-\mathrm{C} 20-\mathrm{C} 21-\mathrm{C} 22$ & $-59.5(2)$ \\
\hline $\mathrm{Fe} 2-\mathrm{C} 20-\mathrm{C} 24-\mathrm{C} 23$ & $59.7(3)$ \\
\hline $\mathrm{Fe} 2-\mathrm{C} 21-\mathrm{C} 22-\mathrm{C} 23$ & $-59.4(3)$ \\
\hline $\mathrm{Fe} 2-\mathrm{C} 22-\mathrm{C} 23-\mathrm{C} 24$ & $-59.0(3)$ \\
\hline $\mathrm{Fe} 2-\mathrm{C} 23-\mathrm{C} 24-\mathrm{C} 20$ & $-59.8(3)$ \\
\hline $\mathrm{S} 1-\mathrm{P} 1-\mathrm{C} 1-\mathrm{Fe} 1$ & $61.4(2)$ \\
\hline $\mathrm{S} 1-\mathrm{P} 1-\mathrm{C} 1-\mathrm{C} 2$ & $-32.1(3)$ \\
\hline $\mathrm{S} 1-\mathrm{P} 1-\mathrm{C} 1-\mathrm{C} 5$ & $150.8(2)$ \\
\hline $\mathrm{S} 1-\mathrm{P} 1-\mathrm{C} 15-\mathrm{Fe} 2$ & $48.3(2)$ \\
\hline $\mathrm{S} 1-\mathrm{P} 1-\mathrm{C} 15-\mathrm{C} 16$ & $-45.9(3)$ \\
\hline
\end{tabular}

$\begin{array}{ll}\mathrm{C} 39-\mathrm{C} 34-\mathrm{C} 35 & 119.3(3) \\ \mathrm{C} 34-\mathrm{C} 35-\mathrm{H} 35 & 120.1 \\ \mathrm{C} 36-\mathrm{C} 35-\mathrm{C} 34 & 119.9(4) \\ \mathrm{C} 36-\mathrm{C} 35-\mathrm{H} 35 & 120.1 \\ \mathrm{C} 35-\mathrm{C} 36-\mathrm{H} 36 & 120.0 \\ \mathrm{C} 37-\mathrm{C} 36-\mathrm{C} 35 & 120.1(4) \\ \mathrm{C} 37-\mathrm{C} 36-\mathrm{H} 36 & 120.0 \\ \mathrm{C} 36-\mathrm{C} 37-\mathrm{H} 37 & 119.5 \\ \mathrm{C} 36-\mathrm{C} 37-\mathrm{C} 38 & 120.9(4) \\ \mathrm{C} 38-\mathrm{C} 37-\mathrm{H} 37 & 119.5 \\ \mathrm{C} 37-\mathrm{C} 38-\mathrm{H} 38 & 120.2 \\ \mathrm{C} 37-\mathrm{C} 38-\mathrm{C} 39 & 119.6(4) \\ \mathrm{C} 39-\mathrm{C} 38-\mathrm{H} 38 & 120.2 \\ \mathrm{C} 34-\mathrm{C} 39-\mathrm{C} 38 & 120.2(3) \\ \mathrm{C} 34-\mathrm{C} 39-\mathrm{H} 39 & 119.9 \\ \mathrm{C} 38-\mathrm{C} 39-\mathrm{H} 39 & 119.9 \\ \end{array}$

C6- 7 - 8 - $-\mathrm{C} 9 \quad 0.1(5)$

$\mathrm{C} 7-\mathrm{C} 6-\mathrm{C} 10-\mathrm{Fe} 1 \quad-59.8(2)$

$\mathrm{C} 7-\mathrm{C} 6-\mathrm{C} 10-\mathrm{C} 9 \quad-0.7$ (4)

$\mathrm{C} 7-\mathrm{C} 8-\mathrm{C} 9-\mathrm{Fe} 1 \quad 59.0(3)$

$\mathrm{C} 7-\mathrm{C} 8-\mathrm{C} 9-\mathrm{C} 10 \quad-0.6(5)$

$\mathrm{C} 8-\mathrm{C} 9-\mathrm{C} 10-\mathrm{Fe} 1 \quad 59.5$ (3)

$\mathrm{C} 8-\mathrm{C} 9-\mathrm{C} 10-\mathrm{C} 6 \quad 0.8$ (4)

$\mathrm{C} 10-\mathrm{C} 6-\mathrm{C} 7-\mathrm{Fe} 1 \quad 60.3(2)$

$\mathrm{C} 10-\mathrm{C} 6-\mathrm{C} 7-\mathrm{C} 8 \quad 0.3(4)$

$\mathrm{C} 11-\mathrm{C} 2-\mathrm{C} 3-\mathrm{Fe} 1 \quad 119.0(4)$

$\mathrm{C} 11-\mathrm{C} 2-\mathrm{C} 3-\mathrm{C} 4 \quad 178.4(3)$

$\mathrm{C} 15-\mathrm{P} 1-\mathrm{C} 1-\mathrm{Fe} 1 \quad-169.37(18)$

$\mathrm{C} 15-\mathrm{P} 1-\mathrm{C} 1-\mathrm{C} 2 \quad 97.1(3)$

$\mathrm{C} 15-\mathrm{P} 1-\mathrm{C} 1-\mathrm{C} 5 \quad-80.0(3)$

$\mathrm{C} 15-\mathrm{P} 1-\mathrm{C} 34-\mathrm{C} 35 \quad-146.0$ (3)

$\mathrm{C} 15-\mathrm{P} 1-\mathrm{C} 34-\mathrm{C} 39 \quad 31.5(3)$

$\mathrm{C} 15-\mathrm{C} 16-\mathrm{C} 17-\mathrm{Fe} 2 \quad-58.0$ (2)

$\mathrm{C} 15-\mathrm{C} 16-\mathrm{C} 17-\mathrm{C} 18 \quad 0.7$ (3)

$\mathrm{C} 15-\mathrm{C} 16-\mathrm{C} 25-\mathrm{N} 1 \quad-81.1(4)$

$\mathrm{C} 15-\mathrm{C} 16-\mathrm{C} 25-\mathrm{C} 33 \quad 158.8$ (3)

$\mathrm{C} 16-\mathrm{C} 15-\mathrm{C} 19-\mathrm{Fe} 2 \quad 60.2(2)$

$\mathrm{C} 16-\mathrm{C} 15-\mathrm{C} 19-\mathrm{C} 18 \quad 0.7(3)$

$\mathrm{C} 16-\mathrm{C} 17-\mathrm{C} 18-\mathrm{Fe} 2 \quad-58.9(2)$

$\mathrm{C} 16-\mathrm{C} 17-\mathrm{C} 18-\mathrm{C} 19 \quad-0.3$ (4)

$\mathrm{C} 17-\mathrm{C} 16-\mathrm{C} 25-\mathrm{N} 1 \quad 93.2(4)$

$\mathrm{C} 17-\mathrm{C} 16-\mathrm{C} 25-\mathrm{C} 33 \quad-26.9(4)$

$\mathrm{C} 17-\mathrm{C} 18-\mathrm{C} 19-\mathrm{Fe} 2 \quad-58.9$ (2)

$\mathrm{C} 17-\mathrm{C} 18-\mathrm{C} 19-\mathrm{C} 15 \quad-0.2$ (4)

$\mathrm{C} 19-\mathrm{C} 15-\mathrm{C} 16-\mathrm{Fe} 2 \quad-59.9(2)$

$\mathrm{C} 19-\mathrm{C} 15-\mathrm{C} 16-\mathrm{C} 17-0.9$ (3) 


$\begin{array}{ll}\mathrm{S} 1-\mathrm{P} 1-\mathrm{C} 15-\mathrm{C} 19 & 139.1(2) \\ \mathrm{S} 1-\mathrm{P} 1-\mathrm{C} 34-\mathrm{C} 35 & -21.1(3) \\ \mathrm{S} 1-\mathrm{P} 1-\mathrm{C} 34-\mathrm{C} 39 & 156.5(2) \\ \mathrm{P} 1-\mathrm{C} 1-\mathrm{C} 2-\mathrm{F} 1 & 122.3(3) \\ \mathrm{P} 1-\mathrm{C} 1-\mathrm{C} 2-\mathrm{C} 3 & -178.6(3) \\ \mathrm{P} 1-\mathrm{C} 1-\mathrm{C} 2-\mathrm{C} 11 & 4.2(5) \\ \mathrm{P} 1-\mathrm{C} 1-\mathrm{C} 5-\mathrm{Fe} 1 & -122.3(2) \\ \mathrm{P} 1-\mathrm{C} 1-\mathrm{C} 5-\mathrm{C} 4 & 178.4(2) \\ \mathrm{P} 1-\mathrm{C} 15-\mathrm{C} 16-\mathrm{Fe} 2 & 124.4(2) \\ \mathrm{P} 1-\mathrm{C} 15-\mathrm{C} 16-\mathrm{C} 17 & -176.5(2) \\ \mathrm{P} 1-\mathrm{C} 15-\mathrm{C} 16-\mathrm{C} 25 & -1.3(5) \\ \mathrm{P} 1-\mathrm{C} 15-\mathrm{C} 19-\mathrm{Fe} 2 & -124.0(2) \\ \mathrm{P} 1-\mathrm{C} 15-\mathrm{C} 19-\mathrm{C} 18 & 176.5(2) \\ \mathrm{P} 1-\mathrm{C} 34-\mathrm{C} 35-\mathrm{C} 36 & 178.2(3) \\ \mathrm{P} 1-\mathrm{C} 34-\mathrm{C} 39-\mathrm{C} 38 & -177.5(3) \\ \mathrm{N} 1-\mathrm{C} 26-\mathrm{C} 27-\mathrm{C} 28 & 131.4(4) \\ \mathrm{N} 1-\mathrm{C} 26-\mathrm{C} 27-\mathrm{C} 32 & -49.0(5) \\ \mathrm{C} 1-\mathrm{P} 1-\mathrm{C} 15-\mathrm{Fe} 2 & -81.2(2) \\ \mathrm{C} 1-\mathrm{P} 1-\mathrm{C} 15-\mathrm{C} 16 & -175.4(3) \\ \mathrm{C} 1-\mathrm{P} 1-\mathrm{C} 15-\mathrm{C} 19 & 9.6(3) \\ \mathrm{C} 1-\mathrm{P} 1-\mathrm{C} 34-\mathrm{C} 35 & 105.6(3) \\ \mathrm{C} 1-\mathrm{P} 1-\mathrm{C} 34-\mathrm{C} 39 & -76.9(3) \\ \mathrm{C} 1-\mathrm{C} 2-\mathrm{C} 3-\mathrm{Fe} 1 & -58.3(2) \\ \mathrm{C} 1-\mathrm{C} 2-\mathrm{C} 3-\mathrm{C} 4 & 1.1(4) \\ \mathrm{C} 1-\mathrm{C} 2-\mathrm{C} 11-\mathrm{C} 12 & -157.0(4) \\ \mathrm{C} 2-\mathrm{C} 1-\mathrm{C} 5-\mathrm{Fe} 1 & 60.1(2) \\ \mathrm{C} 2-\mathrm{C} 1-\mathrm{C} 5-\mathrm{C} 4 & 0.8(4) \\ \mathrm{C} 2-\mathrm{C} 3-\mathrm{C} 4-\mathrm{Fe} 1 & -59.0(2) \\ \mathrm{C} 2-\mathrm{C} 3-\mathrm{C} 4-\mathrm{C} 5 & -0.7(4) \\ \mathrm{C} 3-\mathrm{C} 2-\mathrm{C} 11-\mathrm{C} 12 & 26.3(6) \\ \mathrm{C} 3-\mathrm{C} 4-\mathrm{C} 5-\mathrm{Fe} 1 & -58.3(2) \\ \mathrm{C} 3-\mathrm{C} 4-\mathrm{C} 5-\mathrm{C} 1 & -0.1(4) \\ \mathrm{C} 5-\mathrm{C} 1-\mathrm{C} 2-\mathrm{Fe} 1 & -60.3(2) \\ \mathrm{C} 5-\mathrm{C} 1-\mathrm{C} 2-\mathrm{C} 3 & -1.1(4) \\ \mathrm{C} 5-\mathrm{C} 1-\mathrm{C} 2-\mathrm{C} 11 & -178.4(3) \\ \mathrm{C} 6-\mathrm{C} 7-\mathrm{C} 8-\mathrm{Fe} 1 & 59.5(3) \\ & \end{array}$

$\begin{array}{ll}\mathrm{C} 19-\mathrm{C} 15-\mathrm{C} 16-\mathrm{C} 25 & 174.3(3) \\ \mathrm{C} 20-\mathrm{C} 21-\mathrm{C} 22-\mathrm{Fe} 2 & 59.3(2) \\ \mathrm{C} 20-\mathrm{C} 21-\mathrm{C} 22-\mathrm{C} 23 & -0.1(4) \\ \mathrm{C} 21-\mathrm{C} 20-\mathrm{C} 24-\mathrm{Fe} 2 & -59.3(2) \\ \mathrm{C} 21-\mathrm{C} 20-\mathrm{C} 24-\mathrm{C} 23 & 0.4(4) \\ \mathrm{C} 21-\mathrm{C} 22-\mathrm{C} 23-\mathrm{Fe} 2 & 59.4(3) \\ \mathrm{C} 21-\mathrm{C} 22-\mathrm{C} 23-\mathrm{C} 24 & 0.4(4) \\ \mathrm{C} 22-\mathrm{C} 23-\mathrm{C} 24-\mathrm{Fe} 2 & 59.3(3) \\ \mathrm{C} 22-\mathrm{C} 23-\mathrm{C} 24-\mathrm{C} 20 & -0.5(4) \\ \mathrm{C} 24-\mathrm{C} 20-\mathrm{C} 21-\mathrm{Fe} 2 & 59.3(3) \\ \mathrm{C} 24-\mathrm{C} 20-\mathrm{C} 21-\mathrm{C} 22 & -0.2(4) \\ \mathrm{C} 25-\mathrm{N} 1-\mathrm{C} 26-\mathrm{C} 27 & 177.9(3) \\ \mathrm{C} 25-\mathrm{C} 16-\mathrm{C} 17-\mathrm{Fe} 2 & 126.7(3) \\ \mathrm{C} 25-\mathrm{C} 16-\mathrm{C} 17-\mathrm{C} 18 & -174.5(3) \\ \mathrm{C} 26-\mathrm{N} 1-\mathrm{C} 25-\mathrm{C} 16 & 156.5(3) \\ \mathrm{C} 26-\mathrm{N} 1-\mathrm{C} 25-\mathrm{C} 33 & -81.7(4) \\ \mathrm{C} 26-\mathrm{C} 27-\mathrm{C} 28-\mathrm{C} 29 & -179.9(3) \\ \mathrm{C} 26-\mathrm{C} 27-\mathrm{C} 32-\mathrm{C} 31 & -178.3(4) \\ \mathrm{C} 27-\mathrm{C} 28-\mathrm{C} 29-\mathrm{C} 30 & -2.1(6) \\ \mathrm{C} 28-\mathrm{C} 27-\mathrm{C} 32-\mathrm{C} 31 & 1.3(5) \\ \mathrm{C} 28-\mathrm{C} 29-\mathrm{C} 30-\mathrm{C} 31 & 1.9(6) \\ \mathrm{C} 29-\mathrm{C} 30-\mathrm{C} 31-\mathrm{C} 32 & -0.1(6) \\ \mathrm{C} 30-\mathrm{C} 31-\mathrm{C} 32-\mathrm{C} 27 & -1.5(6) \\ \mathrm{C} 32-\mathrm{C} 27-\mathrm{C} 28-\mathrm{C} 29 & 0.5(6) \\ \mathrm{C} 34-\mathrm{P} 1-\mathrm{C} 1-\mathrm{Fe} 1 & -62.2(2) \\ \mathrm{C} 34-\mathrm{P} 1-\mathrm{C} 1-\mathrm{C} 2 & -155.8(3) \\ \mathrm{C} 34-\mathrm{P} 1-\mathrm{C} 1-\mathrm{C} 5 & 27.1(3) \\ \mathrm{C} 34-\mathrm{P} 1-\mathrm{C} 15-\mathrm{Fe} 2 & 170.4(2) \\ \mathrm{C} 34-\mathrm{P} 1-\mathrm{C} 15-\mathrm{C} 16 & 76.2(3) \\ \mathrm{C} 34-\mathrm{P} 1-\mathrm{C} 15-\mathrm{C} 19 & -98.8(3) \\ \mathrm{C} 34-\mathrm{C} 35-\mathrm{C} 36-\mathrm{C} 37 & -1.4(6) \\ \mathrm{C} 35-\mathrm{C} 34-\mathrm{C} 39-\mathrm{C} 38 & 0.0(5) \\ \mathrm{C} 35-\mathrm{C} 36-\mathrm{C} 37-\mathrm{C} 38 & 1.6(6) \\ \mathrm{C} 36-\mathrm{C} 37-\mathrm{C} 38-\mathrm{C} 39 & -1.0(6) \\ \mathrm{C} 37-\mathrm{C} 38-\mathrm{C} 39-\mathrm{C} 34 & 0.2(6) \\ \mathrm{C} 39-\mathrm{C} 34-\mathrm{C} 35-\mathrm{C} 36 & 0.6(5) \\ & \end{array}$

